Florida International University FIU Digital Commons

$3-25-2015$

\title{
Large-Scale Testing to Study the Effects of Critical Parameters on the Aerodynamic Behavior of Long Span Bridges
}

Ramtin Kargarmoakhar

rkarg001@fiu.edu

DOI: $10.25148 /$ etd.FI15032184

Follow this and additional works at: https://digitalcommons.fiu.edu/etd

Part of the Civil Engineering Commons, and the Structural Engineering Commons

\section{Recommended Citation}

Kargarmoakhar, Ramtin, "Large-Scale Testing to Study the Effects of Critical Parameters on the Aerodynamic Behavior of Long Span Bridges" (2015). FIU Electronic Theses and Dissertations. 1857.

https://digitalcommons.fiu.edu/etd/1857 


\title{
FLORIDA INTERNATIONAL UNIVERSITY
}

Miami, Florida

\section{LARGE-SCALE TESTING TO STUDY THE EFFECTS OF CRITICAL}

PARAMETERS ON THE AERODYNAMIC BEHAVIOR OF LONG SPAN BRIDGES

\author{
A dissertation submitted in partial fulfillment of \\ the requirements for the degree of \\ DOCTOR OF PHILOSOPHY \\ in \\ CIVIL ENGINEERING \\ by \\ Ramtin Kargarmoakhar
}


To: Dean Amir Mirmiran

College of Engineering and Computing

This dissertation, written by Ramtin Kargarmoakhar, and entitled Large-Scale Testing to Study the Effects of Critical Parameters on the Aerodynamic Behavior of Long Span Bridges, having been approved in respect to style and intellectual content, is referred to you for judgment.

We have read this dissertation and recommend that it be approved.

Nakin Suksawang

Irtishad Ahmad

Ioannis Zisis

Amir Mirmiran

Peter Irwin, Co-Major Professor

Arindam Gan Chowdhury, Co-Major Professor

Date of Defense: March 25, 2015

The dissertation of Ramtin Kargarmoakhar is approved.

Dean Amir Mirmiran

College of Engineering and Computing

Dean Lakshmi N. Reddi

University Graduate School

Florida International University, 2015 
C Copyright 2015 by Ramtin Kargarmoakhar

All rights reserved. 


\section{DEDICATION}

This dissertation is dedicated to my wife, Maryam Asghari Mooneghi. There is an old quote saying that "No man succeeds without a good woman behind him". I should say that this is more than true in my case. My wife not only was my support as my best friend during my career, let me share with her the difficulties I faced and helped me relax, but also was a great scientist with whom I was able to discuss about challenging problems. There were some days I was near to a complete surrender, but her sacrifices let me stand up again and continue the way. I am always grateful to my wife, not only as my wife but as my closest friend and I want to thank her for her supports during the precious time that we spent together at FIU and at Wall of Wind to get our PhDs.

To my dear parents, Dr. Rohani Kargarmoakhar and Mrs. Masrooreh Hashemi Zonoz. I am always grateful to my mom, for her endless love, support and encouragement. My father's achievements in science, as one of the greatest scientists in his field in the world, have always been a motivation for me to study and excel in my career.

To my brother and sister, Roozbeh and Maral, for being the pillows, catapults, cheerleading squad and sounding boards I have needed.

And finally to my lovely in-laws, Mr. Aboumohammad Asghari Mooneghi, Mrs. Soheila Esmaeeli Tehrani, Sarah, Nargess and Shiva who have always been on my side when I needed. I dedicate my dissertation deeply from my heart to my Father in-law, Mr. Aboumohammad Asghari Mooneghi who I lost recently but is still alive in my heart and I can never forget his supportive smiles for my achievements. 


\section{ACKNOWLEDGMENTS}

First and foremost, I would like to thank my major professor Dr. Arindam Gan Chowdhury for his supervision, valuable advice, crucial contribution, and patience which made this dissertation possible. There are not enough words to express my deep and sincere appreciation.

I express my appreciation to my second major professor, Dr. Peter Irwin. I am very grateful for his support, guidance, and valuable contribution. Without his guidance and persistent help this dissertation would not have been possible.

Sincere thanks to the members of my doctoral committee, Dr. Amir Mirmiran, Dr. Ioannis Zisis, Dr. Irtishad Ahmad, and Dr. Nakin Suksawang for their input, valuable discussions and accessibility.

The Wall of Wind research performed was supported by the National Science Foundation (NSF Award No. CMMI-0928740 and CMMI-1151003), Florida Sea Grant College Program (Project \# R/C-D-19-FIU), and Center of Excellence in Hurricane Damage Mitigation and Product Development. WOW instrumentation has been supported through the NSF MRI Award No. CMMI-0923365. The support from the International Hurricane Research Center (IHRC) at FIU, Department of Energy, Florida Division of Emergency Management is greatly acknowledged. I would like to thank T.Y. Lin International for providing information on the East Bay Bridge. The unreserved input during tests offered by the Wall of Wind manager, Walter Conklin and the research scientists, Roy Liu Marquis, is greatly acknowledged. 


\begin{abstract}
OF THE DISSERTATION
LARGE-SCALE TESTING TO STUDY THE EFFECTS OF CRITICAL PARAMETERS ON THE AERODYNAMIC BEHAVIOR OF LONG SPAN BRIDGES by

Ramtin Kargarmoakhar
\end{abstract}

Florida International University, 2015

Miami, Florida

Professor Arindam Gan Chowdhury, Co-Major Professor

Professor Peter Irwin, Co-Major Professor

Long-span bridges are flexible and therefore are sensitive to wind induced effects. One way to improve the stability of long span bridges against flutter is to use crosssections that involve twin side-by-side decks. However, this can amplify responses due to vortex induced oscillations.

Wind tunnel testing is a well-established practice to evaluate the stability of bridges against wind loads. In order to study the response of the prototype in laboratory, dynamic similarity requirements should be satisfied. One of the parameters that is normally violated in wind tunnel testing is Reynolds number. In this dissertation, the effects of Reynolds number on the aerodynamics of a double deck bridge were evaluated by measuring fluctuating forces on a motionless sectional model of a bridge at different wind speeds representing different Reynolds regimes. Also, the efficacy of vortex mitigation devices was evaluated at different Reynolds number regimes.

One other parameter that is frequently ignored in wind tunnel studies is the correct simulation of turbulence characteristics. Due to the difficulties in simulating flow 
with large turbulence length scale on a sectional model, wind tunnel tests are often performed in smooth flow as a conservative approach. The validity of simplifying assumptions in calculation of buffeting loads, as the direct impact of turbulence, needs to be verified for twin deck bridges. The effects of turbulence characteristics were investigated by testing sectional models of a twin deck bridge under two different turbulent flow conditions.

Not only the flow properties play an important role on the aerodynamic response of the bridge, but also the geometry of the cross section shape is expected to have significant effects. In this dissertation, the effects of deck details, such as width of the gap between the twin decks, and traffic barriers on the aerodynamic characteristics of a twin deck bridge were investigated, particularly on the vortex shedding forces with the aim of clarifying how these shape details can alter the wind induced responses.

Finally, a summary of the issues that are involved in designing a dynamic test rig for high Reynolds number tests is given, using the studied cross section as an example. 


\section{TABLE OF CONTENTS}

CHAPTER

PAGE

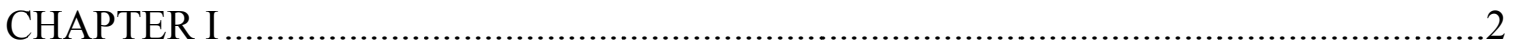

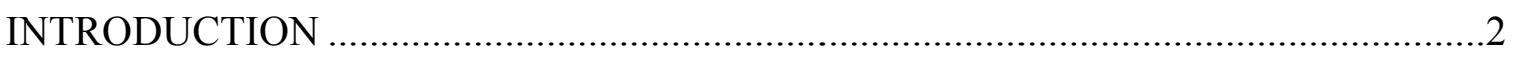

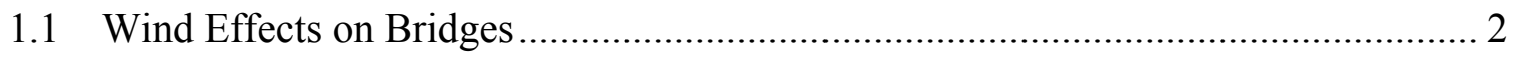

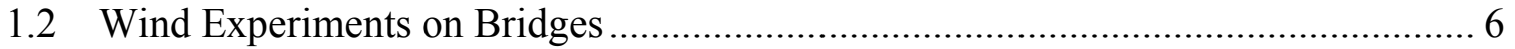

1.2.1 Re effects on wind tunnel testing ................................................................. 11

1.2.2 Turbulence effects on aerodynamic response of bridge model .......................... 13

1.2.3 Effects of geometry and shape details on aerodynamic response of bridges ...... 14

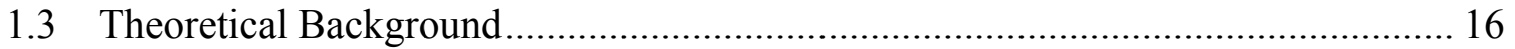

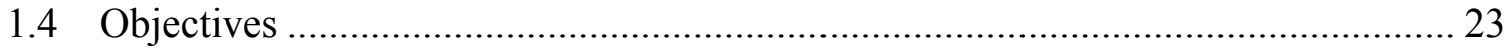

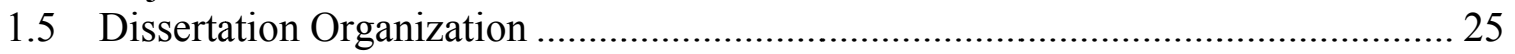

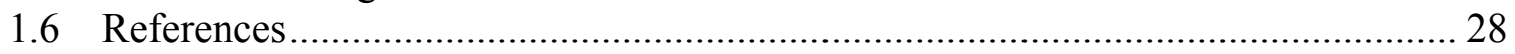

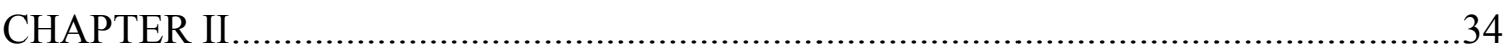

REYNOLDS NUMBER EFFECTS ON TWIN BOX GIRDER LONG SPAN

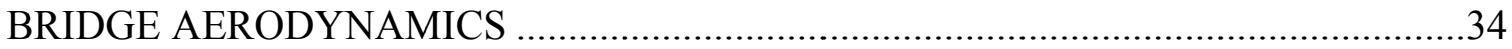

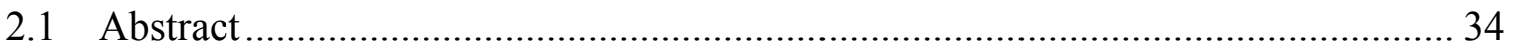

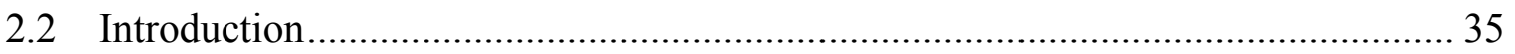

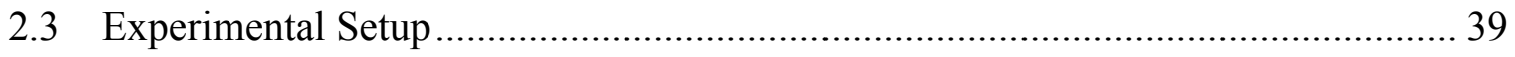

2.3.1 Twin box girder bridge deck ………………............................................. 39

2.3.2 Measurement instrument and test setup ............................................................ 40

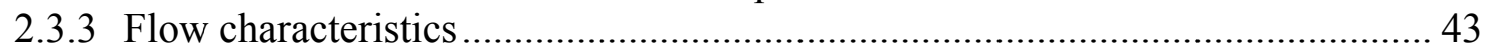

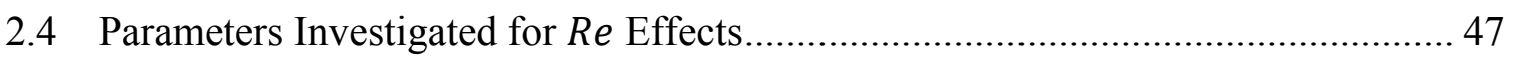

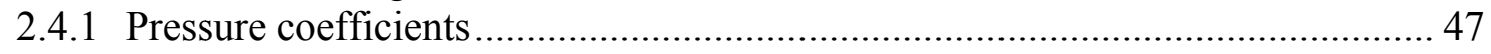

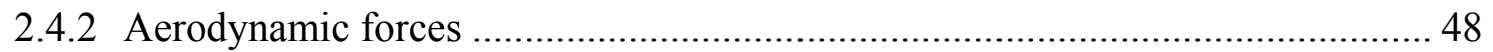

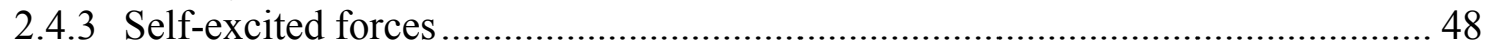

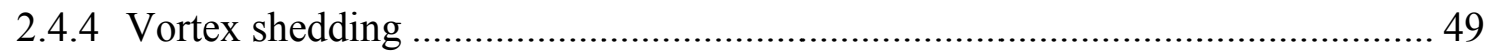

2.5 Experimental Results and Discussion................................................................. 50

2.5.1 Re effects on stream-wise pressure distribution ............................................... 50

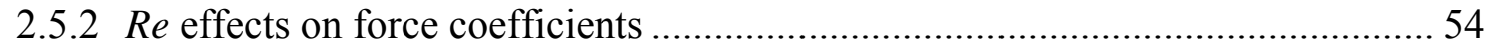

2.5.3 Re effects on aerodynamic load sharing between the two girders...................... 56

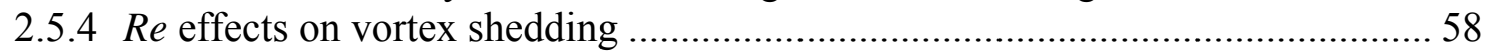

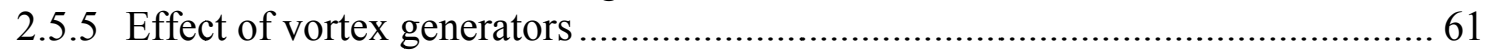

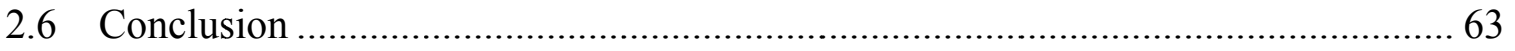

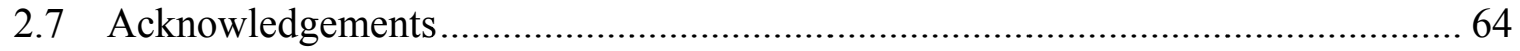

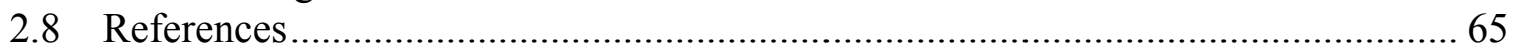

CHAPTER III ...

THE EFFECTS TURBULENCE ON THE PRESSURE DISTRIBUTION AROUND A TWIN BOX GIRDER BRIDGE AND THE RESULTANT AERODYNAMIC FORCES

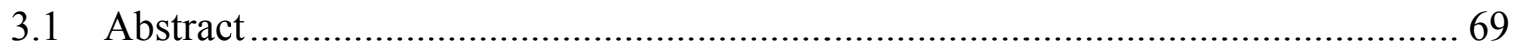

3.2 Background and Literature Review ……………............................................. 70 
3.3 Wind Generating Facility and Wind Flow Characteristics ..................................... 75

3.4 Bridge Deck Section Models and Measuring Instruments ...................................... 78

3.5 Effects of Turbulence Characteristics on Pressure Distribution .............................. 81

3.6 Effects of Turbulence Characteristics on Force Coefficients …………………....... 84

3.7 Effects of Turbulence Characteristics on Vortex Shedding...................................... 90

3.8 Effects of Turbulence Characteristics on Buffeting Loads ...................................... 93

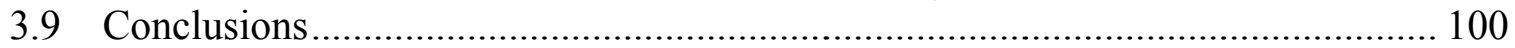

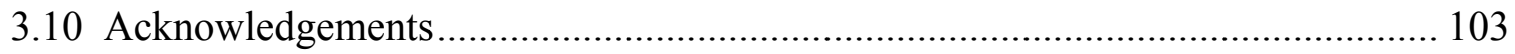

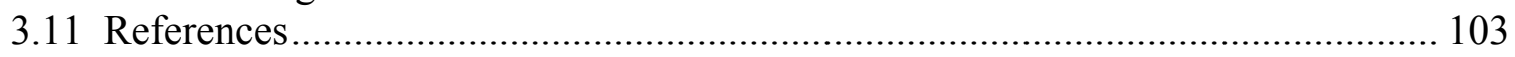

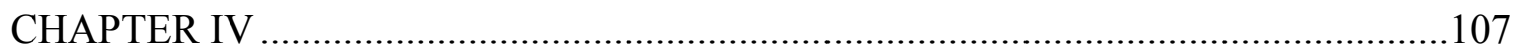

EFFECTS OF DECK DETAILS ON THE TWIN DECK BRIDGE

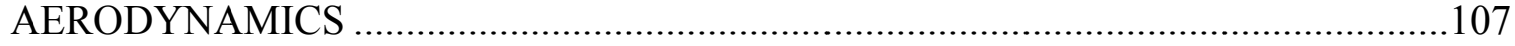

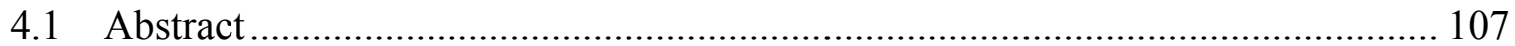

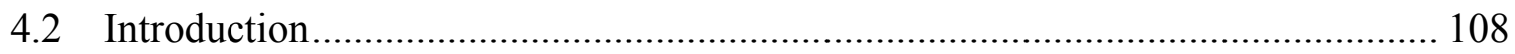

4.3 Description of the Experimental Setup and Testing Procedure .............................. 111

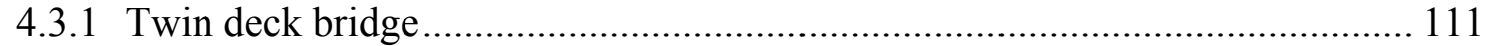

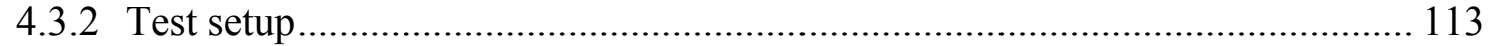

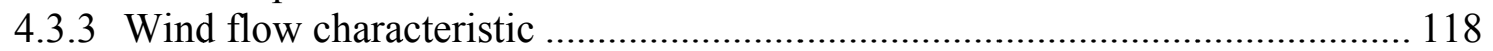

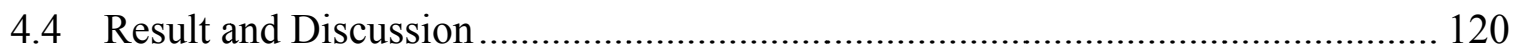

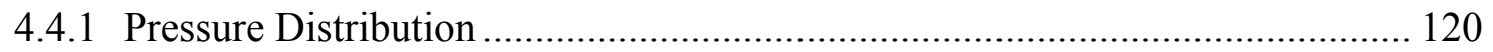

4.4.2 Aerodynamic force coefficients .............................................................. 130

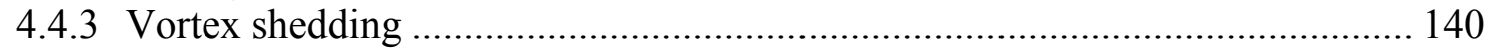

4.5 Summary and Conclusion ........................................................................... 146

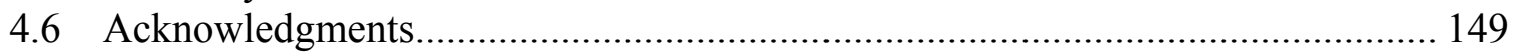

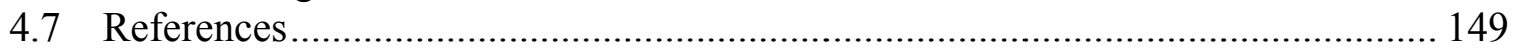

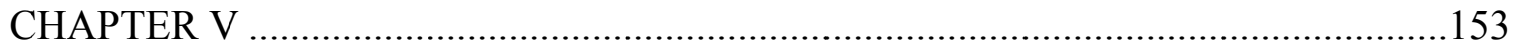

DEVELOPING AN ELASTIC SUSPENSION TEST RIG FOR SECTIONAL

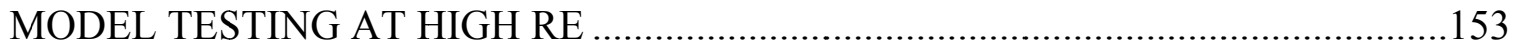

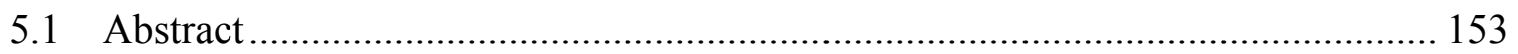

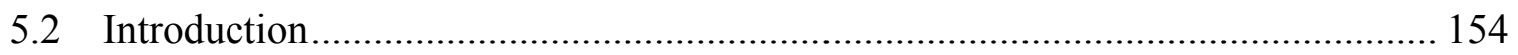

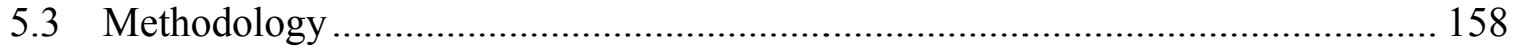

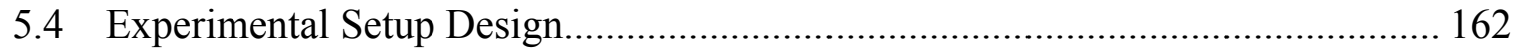

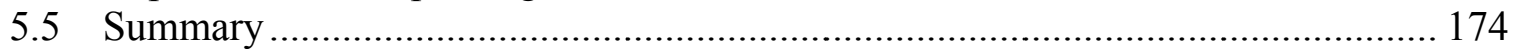

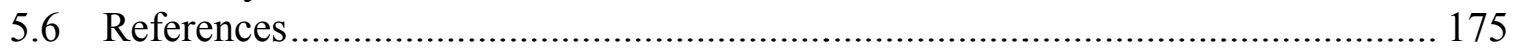

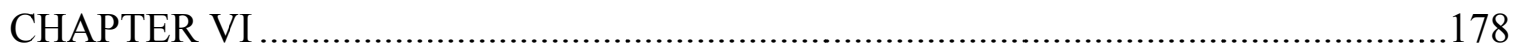

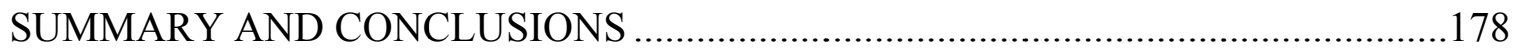

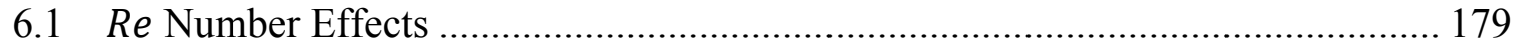

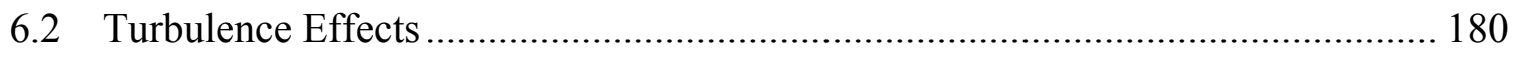

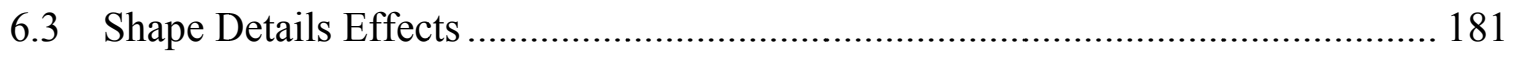

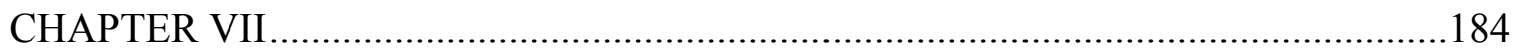

RECOMMENDATIONS FOR FUTURE RESEARCH.............................................184

7.1 Dynamic Tests at High Re to Study Aeroelastic Response................................... 184 
7.2 Comparison of Experimental Results with Analytically Obtained Results

7.3 Investigating the Effect of Additional Shape Details .......................................... 185

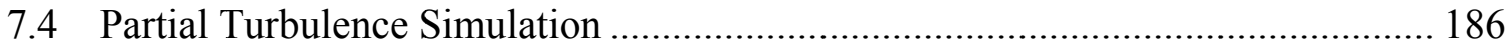

7.5 Buffeting Loads ………………………………......................................... 186

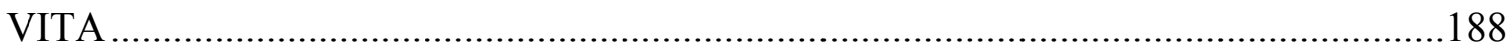




\section{LIST OF TABLES}

TABLE

PAGE

\section{CHAPTER I}

Table 1: Wind induced loads in the vertical direction

\section{CHAPTER II}

Table 1: Test Summary

\section{CHAPTER III}

Table 1: Wind flow characteristics for the two exposures

Table 2: Ratio of the integral length scale to single deck width $\left(L_{u} / b\right)$ for different test conditions.

Table 3: Standard deviation of the fluctuating lift coefficient due to the vortex shedding

\section{CHAPTER IV}

Table 1: Derivative of force and moment coefficients with respect to the angle of attack for the zero wind angle of attack, $R e=4.0 \times 10^{5}$

Table 2: Force and moment coefficients for the zero wind angle of attack from

load cells, $R e=2.6 \times 10^{5}$

\section{CHAPTER V}

Table 1: Table 1 Scaling rules for sectional model testing ...................................... 159

Table 2: Properties of the prototype bridge deck

Table 3: Properties of an extension spring made by Sterling Sprigs L.L.C. 


\section{LIST OF FIGURES}

FIGURE

PAGE

\section{CHAPTER I}

Figure 1: Major wind-induced responses that a bridge deck may experience

Figure 2: Effective forces/moment and wind flow direction for a bridge deck in motion

\section{CHAPTER II}

Figure 1: East span of the San Francisco-Oakland bay bridge

Figure 2: Positions of the pressure taps (model is tilted to $+3^{\circ}$ angle of attack

Figure 3: Testing setup, "A" to "E" show the distribution of the chord-wise

pressure tapped strips along the span length

Figure 4: 1:36 scale model in front of Wall of Wind, Florida International

University.

Figure 5: Cobra Probes Position

Figure 6: Normalized power spectral density (PSD) of the longitudinal component

of the wind speed at bridge height

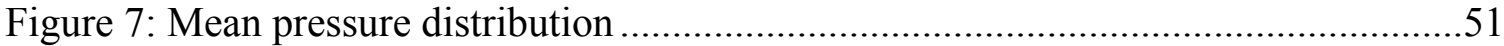

Figure 8: Mean pressure distribution at $+3^{\circ}$ wind angle of attack ................................52

Figure 9: rms of Fluctuating Pressure Distribution...........................................................52

Figure 10: Force coefficients as a function of Reynolds number at $0^{\circ}$ angle of

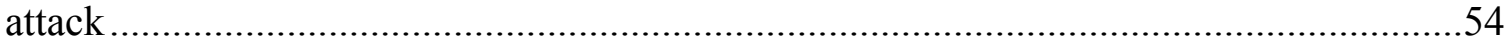

Figure 11: Static aerodynamic coefficients as a function of wind angle of attack ............55

Figure 12: Derivative of force coefficients with respect to the angle of attack .................56

Figure 13: Allotment of each girder from drag.........................................................57

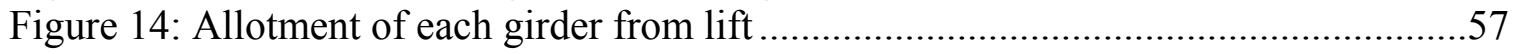

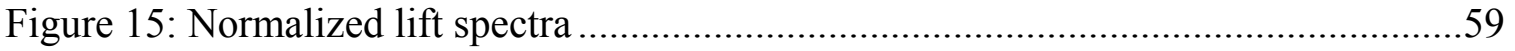

Figure 16: Strouhal number as a function of $R e$.......................................................59

Figure 17: Pressure spectra at $0^{\circ}$ wind angle of attack (Dashed lines show the St

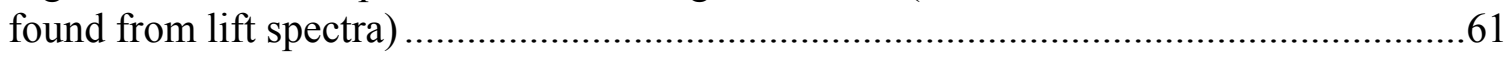

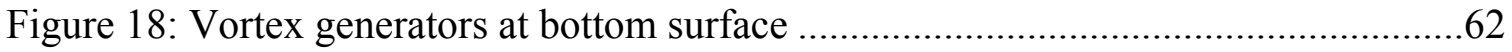

Figure 19: Normalized lift spectra, Bare section ...................................................63

\section{CHAPTER III}

Figure 1: Wall of Wind, Florida International University ..........................................75

Figure 2: Placement of Cobra Probes during free wind speed measurement ...................77

Figure 3: Normalized PSD of the wind fluctuations.................................................... 77

Figure 4: East Bay Bridge deck information ...................................................... 78

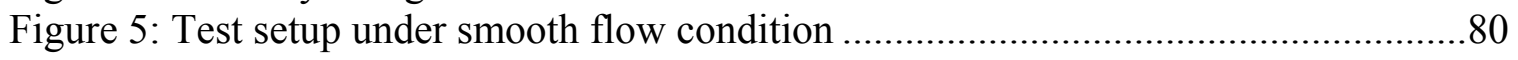

Figure 6: Chord-wise distribution of the pressure taps around the section model.............81

Figure 7: Distribution of the pressure tapped chord-wise strips along the span length. 
Figure 8: Mean pressure coefficient distribution, 1/36 scale model, $R e=1.7 \times$ $10^{6}$

Figure 9: Mean pressure coefficient distribution, $R e=1.7 \times 10^{6}$

Figure 10: Force and moment coefficients as a function of Reynolds number at $0^{\circ}$

angle of attack

Figure 11: Force coefficients as a function of reduced turbulence intensity for $0^{\circ}$ angle of attack, $\circ R e=1.3 \times 10^{6} \square R e=1.7 \times 10^{6}$

Figure 12: Forces and moment coefficients derivatives with respect to the angle of

attack for the zero wind angle of attack

Figure 13: Lift coefficient power spectral density at $R e=1.7 \times 10^{6}$,

Figure 14: Lift coefficient power spectral density at $-6^{\circ}$ wind angle of attack

$\left(R e=1.7 \times 10^{6}\right)$

Figure 15: Standard deviation of the fluctuating lift coefficient due to vortex

shedding for $-6^{\circ}$ wind angle of attack

Figure 16: Lift aerodynamic admittance vs. reduced frequency $(f / B U), R e=$ $1.7 \times 10^{6}$

Figure 17: Span-wise cross-correlation coefficient of lift force at $R e=1.7 \times 10^{6} \ldots \ldots . .98$

Figure 18: Co-coherence of the lift forces for $\delta y / b=1.1$ at $R e=1.7 \times 10^{6}$

\section{CHAPTER IV}

Figure 1: East span of the San Francisco-Oakland bay bridge

Figure 2: Test setup in front of the Wall of Wind.

Figure 3: Test setup for studying the gap width effects 115

Figure 4: Distribution of strips of pressure taps.

Figure 5: Positions of the pressure taps

Figure 6: Test setup for studying the effects of deck appurtenances .............................118

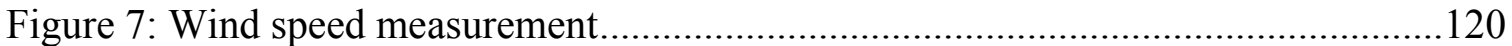

Figure 8: The effect of gap width on the mean pressure distribution ..........................125

Figure 9: The effect of gap width on the fluctuating pressure distribution .....................126

Figure 10: The effect of traffic barriers on the mean pressure distribution .....................128

Figure 11: The effect of traffic barriers on the fluctuating pressure distribution ............129

Figure 12: The effect of bike path on the mean pressure distribution ..........................130

Figure 13: Force and moment coefficients for the model with the largest gap

width $(\mathrm{b} / \mathrm{C}=1.00), R e=1.4 \times 10^{5}, \rightarrow$ Load cells, $\cdots . . .$. Pressure measurement ..............134

Figure 14: The effect of gap width on the force and moment coefficients ....................135

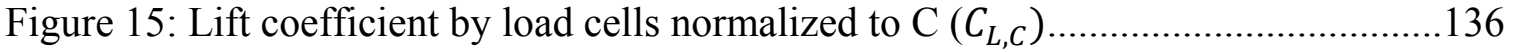

Figure 16: Derivative of force and moment coefficients with respect to the angle

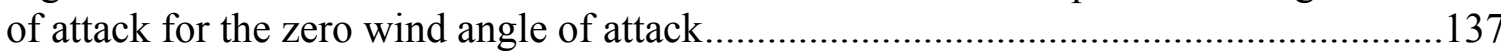

Figure 17:The effect of traffic barriers on the force and moment coefficients...............139

Figure 18: Lift coefficient spectra for the zero wind angle of attack............................142

Figure 19: Strouhal Number as a function of gap to single deck width ratio (b/C)........143

Figure 20: Lift coefficient spectra for the zero wind angle of attack............................144

Figure 21: Lift coefficient spectra for the zero wind angle of attack............................144

Figure 22: Effect of traffic barriers on the lift coefficient spectra ..................................145

Figure 23: Effect of bike path on the normalized lift coefficient spectra ......................146

xiii 


\section{CHAPTER V}

Figure 1: End walls 163

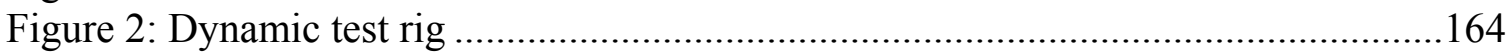

Figure 3: Sectional model to springs connection system................................................165

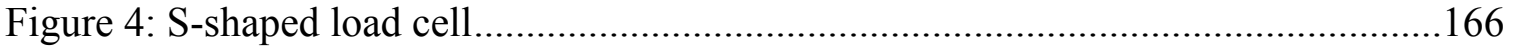

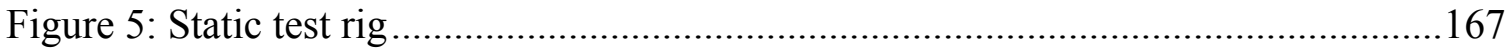

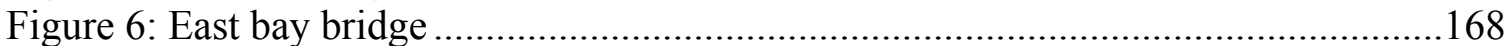

Figure 7: Simplified cross sectional shape of the East bay bridge ................................168

Figure 8: A view of the sectional model...............................................................170

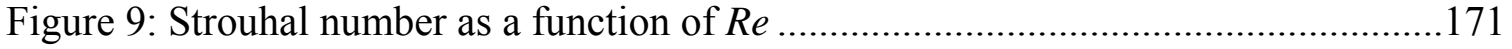


CHAPTER I

INTRODUCTION 


\section{CHAPTER I}

\section{INTRODUCTION}

\subsection{Wind Effects on Bridges}

In the field of structural engineering, wind engineering methods are used to evaluate the interactions between the atmospheric boundary layer wind and manmade structures so that wind-induced loads can be accurately determined, aerodynamic stability assured and serviceability issues due to wind-induced vibrations avoided. The main objective of wind engineering is to evaluate the structural response due to wind-induced loads and come up with strategies to avoid unintended responses. The field of wind engineering covers a wide range of topics including meteorology, fluid dynamics and structural dynamics. The wind-structure interaction can be important for a wide variety of structures, including high rise buildings, towers, bridges and etc. Wind is generated from the movement of the atmospheric air. Aerodynamic and aeroelastic effects constitute the two major wind-structure interaction mechanisms. The aerodynamic effect refers to the fluctuating nature of the wind and its interaction with the structure, whereas aeroelasticity effect refers to the condition wherein aerodynamic forces and structural motions interact significantly. Aerodynamic forces can be divided to two components: the mean value which can be considered as the static part of the wind force and the fluctuating part which is usually referred to as buffeting forces (Davenport, 1961). The same division can be applied to the structural response as it can be divided to mean and fluctuating components.

Since the collapse of Tacoma Narrows Bridge in 1940, the importance of wind effects on long-span bridge design has been acknowledged and this has led to numerous 
researches in this field. During the last decades, many long span bridges have been constructed worldwide. Besides their attractive appearance which makes them to be considered as landmarks, difficulties associated with construction of short span bridges for certain locations have led to a rapid increase in the number of long span bridges. With the same pace, the material science and computer modeling skills have developed such that they have facilitated the design and building of longer span bridges. Longer span structures are more flexible and therefore are more sensitive to wind loads. The more flexible nature of longer span bridges highlights the importance of wind analysis prior to the adoption of the cross-section shape.

The bridge response under wind-induced loads can be grouped into wind loading and stability problems. Among the stability problems, vortex-induced vibration (VIV), across-wind galloping, and flutter are all dynamic instability phenomena and torsional divergence is basically a static instability phenomenon occurring due to a process analogous to column buckling (Simiu and Scanlan, 1996). Galloping and flutter both are driven by self-excited aerodynamic forces. In flutter the aerodynamic forces become large enough to modify the oscillating frequencies of the structure and cancel the mechanical damping effects. This can lead to structural failure. For galloping, flutter and torsional divergence a threshold wind speed exists above which these instabilities occur. However, VIV typically occurs over a narrow band of wind speeds that can be well below the design wind speed.

Vortex shedding occurs during steady uniform flow when alternating vortices are periodically shed into the wake of a structure. The alternating pattern of vortices is commonly referred to as von Kármán vortex street. The alternating shedding of vortices 
produces periodic forces that result in oscillations in a plane normal to the direction of wind flow. Significant oscillation can occur when the frequency of vortex shedding is close to the natural frequency of a flexible structure. These lateral vibrations have a strong organizing effect on the vortex shedding pattern which can increase the strength of the vortices and couple the vortex shedding frequency to the natural frequency of the structure. This phenomenon is known as lock-in and the vibration that occurs due to vortex shedding is called VIV (vortex induced vibration). The amplitude of the vibrations resulting from lock-in is limited by the balance between the energy input into the motion by the vortices and the dissipation of that energy by structural damping. Eventually large oscillations of the structure interfere with the uniform shedding of vortices and therefore the maximum amplitude of vortex induced vibrations may be self-limiting (Blevins, 2001).

Buffeting is a response problem that is defined as the unsteady loading on a structure due to velocity fluctuations in the oncoming flow which causes motions in the structure's various modes of vibration. The buffeting response grows as the wind speed increases and at high velocities the buffeting action contributes significantly to overall wind loading. The estimation of bridge response to the turbulent oncoming flow is typically undertaken only after the aerodynamic stability of the deck cross-section has been assured. However, prediction of the bridge response under buffeting loads is crucial for the design of bridge components.

Figure 1 shows a schematic view of major wind-induced dynamic responses that a long-span bridge deck may experience in a turbulent boundary layer flow. 


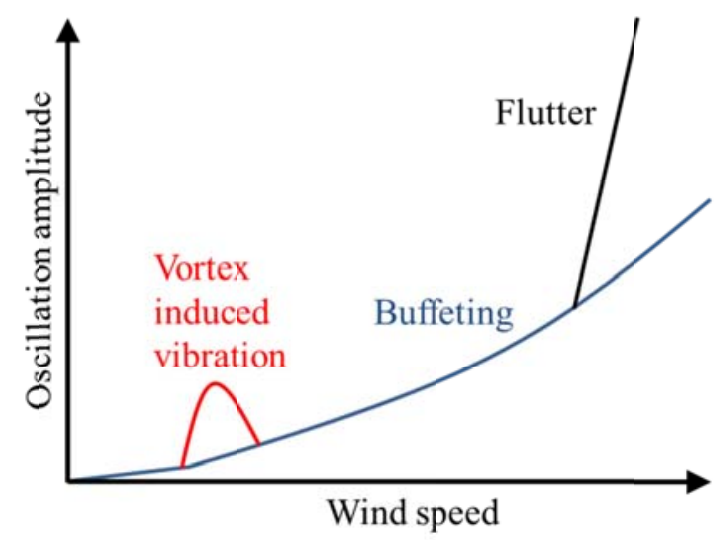

Figure 1: Major wind-induced responses that a bridge deck may experience

Among the dynamic wind-induced responses mentioned above, flutter can lead to catastrophic collapse due to a very rapid increase in amplitude of oscillation once a critical wind speed is exceeded, as shown in Fig. 1. When the wind speed exceeds a particular critical speed, the bridge deck responds exponentially due to the motioninduced instability. Theodorsen (1935) was one of the pioneers to propose an analytical expression in the frequency domain for motion-induced forces on thin airfoil cross sections in aeronautical industry. Scanlan and Tomko (1971) modified this formulation for bridge applications by introducing aerodynamic parameters called flutter derivatives which can be obtained experimentally on a scaled model of the bridge. Extraction of the flutter derivatives from the experiments can be performed by system identification methods that can be found in literature. System identification methods can be categorized into different groups: least squares (LS) (Hsia, 1976), Iterative Least Squares (ILS) (Chowdhury and Sarkar, 2003), unifying least squares (ULS) (Gu et al, 2000; Zhu et al, 2002), instrumental variable (IV) (Imai et al, 1989), maximum likelihood (ML) (Imai et 
al, 1989), extended Kalman filtering (EKF) (Iwamoto and Fujino, 1995), and modified Ibrahim time domain (Sarkar et al, 1992) (MITD).

One way to improve the stability of modern long span bridges against flutter is to use twin side-by-side decks in the design. Sato et al (2002) showed that compared to single girder bridge decks, slotted box girder bridges are more economically efficient and aerodynamically stable. It was also proven by Ge and Xiang (2008) that twin-separated box girders have higher critical flutter speed than single box girders. Several super-long span bridges have adopted this section configuration in the recent years, e.g. the Xihoumen suspension bridge in China, the Stonecutters cable-stayed bridge in Hong Kong, the Gwangyang suspension bridge in Korea, the proposed Messina Strait bridge in Italy and the East Bay bridge in the United States. However, recent literature has showed that introducing a center gap to the bridge deck section shape can increase the susceptibility of the bridge to vortex induced vibration (Kwok et al, 2012). Also, a number of completed bridges have exhibited vortex-induced vibrations under natural winds, e.g. the Volgograd bridge in Russia. There are several cases in the literature showing VIV in prototype bridges (Frandsen, 2001; Fujino and Yoshida, 2002; Larsen et al, 2000).

\subsection{Wind Experiments on Bridges}

Although analytical approaches for investigating wind effects on bridges have developed significantly during the years (Fransos and Bruno, 2010; Kargarmoakhar et al, 2015; Laima et al, 2013), experimentally obtained information still remains a critical component of establishing the aerodynamic loading and stability. This information includes the aeroelastic and aerodynamic parameters that can be obtained from wind 
tunnel testing on a scaled model of the prototype. The application of Computational Fluid Dynamics (CFD) in estimation of forces on oscillating bridge decks is limited because of the difficulty in conducting accurate simulation of complicated turbulent flows in high Reynolds number flow. Therefore, wind tunnel testing plays an important role in evaluating the aerodynamic phenomena that the bridge section experiences under wind loading. The primary purpose of wind tunnel tests is to evaluate the flow field and wind generated loads to predict the structural response and avoid structural instability under these loads.

For civil engineering applications, wind tunnel testing is normally performed in a boundary layer flow that can generate the vertical distribution of velocity at the test section similar to the profile of the wind encountered by prototypes. The full-scale distribution of the wind speed along the height can be estimated from the logarithmic or power law which can be simulated in a wind generating facility, comprising fans, by putting roughness elements and spires upstream of the test models. In reality, civil engineering structures experience fluctuating wind flow in time, resembling a turbulent wind flow. Putting the roughness elements and spires in front of the fans creates fluctuations in flow that can replicate the turbulent flow required for studying the wind effects on civil structures. However, due to the scaling rules, when using sectional models at large enough scale to obtain good geometric accuracy and avoid adverse Reynolds number effects, there is typically a significant mismatch in the ratio of the turbulence integral length scale to bridge width between the model and the prototype. One conservative practice is to evaluate the wind effects on the bridge model under smooth flow. This assumption can yield to larger responses compared to the real structural 
responses of the prototype under natural wind (Wardlaw et al, 1983), but still can be used to ensure the stability of the structure. In order to investigate the bridge response in a turbulent flow using a wind simulating facility that cannot reproduce the turbulence length scale requirements, Partial Turbulence Simulation (PTS) was introduced by Irwin (1998).

The aerodynamic behavior of the bridge can be studied using a motionless sectional model of the prototype under simulated wind conditions in a wind tunnel. However, in order to study the aeroelastic response of the bridge, some of the dynamic properties of the prototype should also be simulated, in addition to satisfaction of geometry similarity. There are three major types of wind tunnel tests that are commonly used in a wind tunnel to explore the aeroelastic behavior of models subjected to wind induced vibration:

- Test on models of the full structure.

- Test on taut-strip models.

- Test on section models.

The type of the testing can be decided based on the size of the test section and the objective of the experiments. Full model testing is the closest option to what happens in reality to the bridge section and can give all the required information about the different aerodynamic and aeroelastic responses. Tests on models of the full structure require that all the prototype details including the geometry and dynamic properties to be scaled and modeled in the experiments. Dynamic similarity can be satisfied by choosing appropriate material properties. Preparing the detailed model with all the scaled properties usually takes a long time and a high cost. Also, due to the relatively small size of the wind 
tunnels, the scaling factor should be chosen large so that the full scaled model of the bridge fits inside the testing section. Therefore, simulating the deck details becomes harder as the size of the model is very small and also the Reynolds similitude is violated by a larger margin as the model scale becomes very small.

A simpler approach in predicting the bridge response is to perform wind tunnel testing on taut-strip models of the structure. This method is relatively simpler and less expensive compared to the full scaled model testing while it offers most of the advantages of the full aeroelastic model testing. It enables the evaluation of the threedimensional character of the motion of the main span while some other details such as cables and side spans are not modeled. Coupling effects between the vertical and torsional modes can also be modelled for the first fundamental symmetric modes. Using this method, the Froude number similitude can be violated so that it can be used to both study vortex induced vibration at low wind speeds and aerodynamic instabilities at high wind speeds by choosing proper length and time scales independently. In this method, the geometry and mass of the prototype are scaled and modeled using cladding segments which are fixed to tensioned wires or tubes. The tensioned wires provide the required stiffness for the model in the three different degrees of freedom with sinusoidal mode shapes. This method provides information on the responses of the bridge deck in the individual modes of vibration. The data from the wind tunnel tests can then be used in further analyses to predict the complete bridge behavior. Taut-strip model was first introduced by (Davenport et al, 1971) and the details of this approach and the findings of the tests can be found in (Davenport et al, 1971). Taut-strip models can be used for studying the buffeting response of structures under natural winds (Macdonald et al, 2002) 
as well as extracting the aerodynamic characteristics of bridge deck (Davenport et al, 1971; Ma and Chen, 2007). Building a taut-strip model is much simpler than building a full aeroelastic model; however, it is more difficult compared to building a sectional model (Ma and Chen, 2007).

The simplest and most widely used wind experiment on bridges is sectional model testing. While section model testing had been used since the 1940's to evaluate bridge deck stability, the first use of section models specifically to identify flutter derivatives was by Scanlan and Tomko (1971). The basic idea behind this method was an adaptation of Theodoresen's theory (Theodorsen, 1935). In a section model test, a geometrically scaled model of the structure is tested in a wind flow simulating the scaled atmospheric flow conditions. A section model is a two dimensional (2-D) section of the body that is built to a scale to replicate a representative span-wise section of the prototype. The sectional model should be rigid so that the model mode shape remains uniform over its entire length. Sectional model testing can be used both to study the aerodynamic and aeroelastic response of the structure by preventing and allowing the model vibration accordingly. In order to accommodate the model motion for studying the aeroelastic effects, the model is often supported by springs at the ends allowing motions of the rigid model along vertical, torsional and lateral directions. Section model testing is usually performed in the initial stages of the design to detect signs of vortex induced vibration and wind induced instabilities. This method can also be used to determine a wide range of aerodynamic properties ranging from static force coefficients to aerodynamic derivatives. Section modeling often provides fundamental aerodynamic data that is used as a basis for 
comprehensive analytical studies. Scanlan et al (1997) give a summary of comparison between taut-strip model and section model testing.

In order to obtain the actual prototype response under natural winds from experiments, it is required that geometric, kinematic and dynamic similarities to be satisfied between the model and the prototype based on the similitude theory. This means that the shape of the model and topographical features, velocity field, flow pattern and forces generated on the model should be as close to reality as possible, considering the scaling factors (Liu, 1991). Therefore, experimental practice should address this similitude either by matching all the relative parameters completely or by acknowledging the level of uncertainty in conclusions as a result of imperfect simulations. In most cases it is almost impossible to satisfy all the similitude requirements because of the limitations in the test setup and modeling capabilities. Therefore, it is of importance to understand how this violation of similitude can affect the test results.

\subsubsection{Re effects on wind tunnel testing}

One problem is that the normal range of Reynolds number that can be simulated in the majority of wind tunnels is two to three orders of magnitude smaller than the $R e$ range for the prototype. Therefore, the similarity condition with respect to $R e$ cannot be satisfied in ordinary cases. It has been shown that the topology of the flow around some bridge decks changes with Re (Schewe, 2001; Schewe and Larsen, 1998).

Traditionally it is believed that separation points are fixed for wind flow around a bridge, considering the fact that bridges can be considered to be bluff structures. Based on this assumption wind tunnel tests have been performed for smaller $R e$ compared to the real bridge condition. However, recently several studies have demonstrated that 
aerodynamic characteristics and wind response of bridges and generally bluff sections can be influenced by Re (Hui and Larsen, 2002; K. Matsuda, 2001; Larose and D’Auteuil, 2006; Larsen et al, 2008; Schewe, 2001; Schewe and Larsen, 1998).

Schewe and Larsen (1998) studied the flow around a bluff bridge deck in a pressurized wind tunnel capable of reproducing typical model scale $\left(\operatorname{Re} \approx 10^{5}\right)$ and prototype conditions $\left(\operatorname{Re} \approx 10^{7}\right)$. They observed pronounced $R e$ effects in the Strouhal number, and drag coefficients. K. Matsuda (2001) investigated the Re effects on the steady and unsteady aerodynamic forces on a twin-box streamlined bridge deck in a smooth flow. They concluded that conventional wind tunnel test results in the low $R e$ region were conservative for wind resistant-design of bridge decks. Schewe (2001) studied the variation of $\operatorname{Re}\left(10^{4}<\operatorname{Re}<10^{7}\right)$ effects on the aerodynamic properties of a circular cylinder (considered to be a classical bluff body), a sharp-edged, trapezoidalshaped bridge element (considered to be a less bluff body), and a thick airfoil at a high attack angle (acting in between the bluff and stream lined cross section). In all cases, it was concluded that altering the $R e$ value during the tests has led to significant changes in the force coefficients and Strouhal number $(S t)$. This behavior was attributed to the flow structure around the body, the laminar to turbulent transition of the boundary layer/shear layer and its location.

Hui and Larsen (2002) tested the Stonecutters bridge with different scales and concluded that among the force coefficients only the drag coefficient was $R e$ dependent. Also, vortex induced vibrations showed correlation with $R e$ value. Larose and D'Auteuil (2006) investigated the effects of edge sharpness, turbulence and width-to-depth ratio on $R e$ sensitivity for rectangular prisms to parameterize the link between the geometry of a 
bluff body with the sensitivity of its aerodynamics to $R e$. Larsen et al (2008) studied the efficiency of guide vanes used in Stonecutters Bridge for mitigating the vortex excitations for different $R e$. Their results showed that while testing with low $R e$ was unable to demonstrate the efficiency of the guide vanes, increasing $R e$ showed that adding the vanes can suppress the vortex induced vibrations completely. The last two studies show that not only the flow characteristics can change the bridge aerodynamic behavior, but also the section shape and detailing can mitigate or worsen the aerodynamic instability.

\subsubsection{Turbulence effects on aerodynamic response of bridge model}

Researchers have used smooth flow with limited turbulence for their studies assuming that this condition is the worst scenario for aerodynamic instabilities. In reality, long-span bridges can experience turbulence intensities as great as $20 \%$ and integral scales as large as ten times their deck width. This shows the significance of studying the approaching wind turbulence effect. However, due to the limitations associated with the majority of wind tunnels, turbulence characteristics, namely turbulence intensity and turbulence integral length scale, cannot be simulated thoroughly in the experiments. The high cost of simulating every turbulent parameter motivates one to understand the effects of imperfect simulation. Regarding turbulence effects, there are some cases in the literature that show contradictions between different researches (Haan Jr. et al, 1998).

Turbulence parameters include turbulence intensity, turbulence integral scale and turbulence spectra. Several researchers have studied the effects of different wind turbulence characters on long span bridge behavior. One of the first to do so was (Wardlaw et al, 1983) who studied and compared the bridge behavior for different systems with a variety of testing techniques. In general, they concluded that for certain 
types of bridge systems, increasing the turbulence intensity reduced the amplitudes due to vortex excitation. However, their observations were not all consistent and they were not able to explain the reason for all of their observed results. Haan Jr. et al (1998) studied pressure fields around rectangular prisms under turbulent flows of varying scales. According to their results, altering the integral scales of the incident flow changed the mean, rms, and negative peak pressure distributions around the section.

In an analogous study, (Haan and Kareem, 2009) studied the turbulence effect on aeroelastic quantities for an oscillating rectangular prism with a forced-vibration system. To this end, they measured chord-wise distributions of self-excited pressure amplitudes and derived associated phases. They integrated these distributions over the section width and calculated conventional flutter derivatives from numerical integration. According to their results, turbulence was found to have a stabilizing effect on the aerodynamics of the prism. While there are some researches in literature investigating the effect of turbulence on the flutter and buffeting responses of bridge decks (Haan and Kareem, 2009; Larose, 2002; Lin et al, 2003), only limited studies have considered the turbulence effect on VIV (Macdonald et al, 2002; Wardlaw et al, 1983).

According to the author's knowledge, no experimental study has been carried out to consider the simultaneous effect of $R e$ simulation and sensitivity to the turbulence parameters.

\subsubsection{Effects of geometry and shape details on aerodynamic response of bridges}

In order to ensure the aerodynamic stability of bridges, several cross sectional configurations have been proposed in the literature. It has been shown that even a minor 
change in the cross sectional shape can change the aerodynamic response of bridges significantly (Fransos and Bruno, 2010; Jones et al, 1995).

Several researchers have tried to evaluate the effect of the cross sectional shape on the bridge response to the wind loads (Kwok et al, 2012) while some others tried to avoid aerodynamic problems by proposing an inherently superior cross section(Larsen and Wall, 2012). Kwok et al (2012) studied the effects of gap width on the aerodynamic characteristics for a twin-deck bridge in a nominally smooth flow. The results demonstrated that the bridge was susceptible to vortex shedding when a gap spacing was introduced between the two girders, and changing the gap-width significantly altered the excitation mechanism. Larsen and Wall (2012) demonstrated that by choosing a proper angle between the horizontal bottom plate and the inclined side panes for trapezoidal bridge sections, vortex shedding can almost be avoided.

Lin et al (2003) investigated the effect of deck geometry and oncoming turbulence on the flutter and buffeting behavior of cable supported bridges using wind tunnel testing. Their results showed that increasing the width to depth ratio and increasing the free stream turbulence enhanced the bridge's aerodynamic stability. Fransos and Bruno (2010) studied the combined effects of the integral length scale and the lower corner radius of curvature on the flow field around a trapezoidal bridge deck cross-section numerically. They concluded that by making small changes in the parameter values, force coefficients and Strouhal number changed dramatically due to significant modifications in the topological structure of the flow. They suggested that the different $R e$ regimes proposed by Schewe (2001) can happen even for a fixed $R e$ condition, if flow and/or section properties get modified so that the flow pattern changes around the section. 
Results from Jones et al (1995) demonstrated the crucial effects of the minor details such as railings on the aeroelastic response of bridge models.

\subsection{Theoretical Background}

The aerodynamic forces/moment acting on a bridge deck can be expressed in terms of two force components of Drag $\left(F_{D}\right)$ and Lift $\left(F_{L}\right)$ the pitching Moment $(M)$. Figure 2 shows an overview of the effective forces and moment on a bridge deck in motion. Drag coefficient $\left(C_{D}\right)$, Lift coefficient $\left(C_{L}\right)$ and Moment coefficient $\left(C_{M}\right)$ are normalized forms of the aerodynamic force/moments:

$$
C_{D}=\frac{F_{D}}{\frac{1}{2} \rho \bar{V}^{2} H}, C_{L}=\frac{F_{L}}{\frac{1}{2} \rho \bar{V}^{2} B}, C_{M}=\frac{M}{\frac{1}{2} \rho \bar{V}^{2} B^{2}}
$$

where, $F_{D}$ is the mean drag per unit lengh, $F_{L}$ is the mean lift per unit length, $M$ is the mean pitching moment per unit length, $\rho$ is the air density, $\bar{V}$ is the mean wind speed $(\mathrm{m} / \mathrm{s})$, and $B$ and $H$ represent the deck chord and deck height of the model, respectively.

Bridge response under a turbulent wind can be estimated by predicting the wind loads on the bridge from the quasi steady theory and combining the estimated loads with the equations of motion of the deck (Irwin, 1977; Larose, 2002). This method yields a set of formulas which relate the fluctuating wind loads to turbulence fluctuations in the oncoming flow using the steady aerodynamic force coefficients and their derivatives with respect to angle of attack. As a result, the bridge response to the turbulence fluctuations can be worked out. 


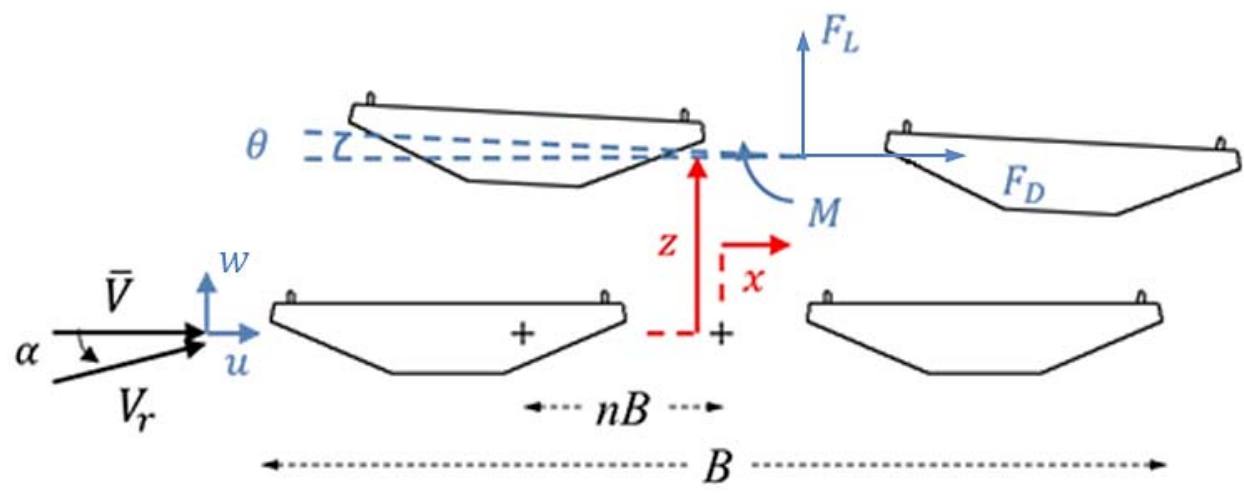

Figure 2: Effective forces/moment and wind flow direction for a bridge deck in motion

In the following, the derivation of the aerodynamic forces in the across wind direction, based on the quasi-steady theory, is explained. Then the calculated forces are combined with the equations of motion to predict the bridge response under a turbulent approaching flow. Based on the results from the across wind direction, the formulations are extended to other degrees of freedom to capture the bridge response in along wind and torsional directions.

Considering the mean wind speed to be equal to $\bar{V}$ and the fluctuating components to be equal to $u$ and $w$ in the along and perpendicular to mean wind speed directions, the relative wind speed $\left(V_{r}\right)$ that an oscillating bridge will perceive can be calculated from:

$$
V_{r}=\sqrt{(\bar{V}+u-\dot{x})^{2}+(w-\dot{z})^{2}}
$$

Assuming that bridge deck displacements and fluctuating wind speeds are small, all the terms including these parameters to the power of two and their multiplication to each other can be neglected. Therefore, relative wind speed $\left(V_{r}\right)$ can be obtained from the simplified equation below.

$$
V_{r}=\sqrt{\bar{V}^{2}+2 \bar{V} u-2 \bar{V} \dot{x}}
$$


For the oscillating bridge, the relative wind angle of attack $(\alpha)$ can be calculated from:

$$
\alpha=\theta+\frac{w-\dot{z}-n B \dot{\theta}}{\bar{V}+u-\dot{x}}
$$

The dotted parameters show the derivative of each quantity with respect to time. $n$ represents the eccentricity between the point of application of the generated aerodynamic force and idealized center of geometry. Irwin (1977) introduced $n B \dot{\theta}$ for bridge decks to express quasi-statically the contribution of the torsional aerodynamic damping in the equation of motion. It was shown that this parameter was not constant for different force components and different shapes (Chen and Kareem, 2002; Neuhaus and Höffer, 2011).

Assuming that force coefficients are linear functions of angle of attack, the force coefficient at each angle of attack $\left(C_{L}(\alpha)\right)$ can be calculated from the force coefficient at zero wind angle of attack $\left(C_{L}(0)\right)$ and the rate of variation of force coefficient with angle of attack $\left(d C_{L} / d \alpha\right)$ from the following equation:

$$
C_{L}(\alpha)=C_{L}(0)+\alpha \times\left(d C_{L} / d \alpha\right)
$$

Considering the motion of the bridge deck and the resulting relative wind speed, the lift force per unit length can be obtained from the following equation:

$$
F_{L}=1 / 2 \rho B V_{r}^{2} \times C_{L}(\alpha)
$$

From Eqs. 3 to 6, the lift force per unit length can be calculated from the simplified equation below:

$$
\begin{gathered}
F_{L}=\rho B / 2\left(C_{L}(0) \bar{V}^{2}+2 C_{L}(0) \bar{V} u+\omega \bar{V} d C_{L} /_{d \alpha}-\dot{z} \bar{V} d C_{L} /_{d \alpha}-2 C_{L}(0) \bar{V} \dot{x}\right. \\
\left.+\theta \bar{V}^{2} d C_{L} /_{d \alpha}-n B \dot{\theta} \bar{V} d C_{L} / d \alpha\right)
\end{gathered}
$$


The generated forces can be categorized into mean wind loads, the buffeting wind loads, the motion induced loads (corresponding to aerodynamic damping) and coupling loads (factor of displacements in the other directions), as follows:

Table 1: Wind induced loads in the vertical direction

\begin{tabular}{|l|c|}
\hline Mean wind loads & $1 / 2 \rho B \bar{V}^{2} C_{L}(0)$ \\
\hline Buffeting wind loads & $\rho \bar{V} B / 2\left[2 C_{L} u+\omega d C_{L} / d \alpha\right]$ \\
\hline $\begin{array}{l}\text { Motion induced loads } \\
\text { (Damping) }\end{array}$ & $-1 / 2 \rho B \bar{V} \dot{z} d C_{L} / d \alpha$ \\
\hline Coupling loads & $\rho \bar{V} B / 2\left[-2 C_{L}(0) \dot{x}+\theta \bar{V} d C_{L} / d \alpha-n B \dot{\theta} d C_{L} / d \alpha\right]$ \\
\hline
\end{tabular}

A similar approach can be applied to the along wind and torsional directions. Combining the equations of motion with wind induced loads and transferring all the wind induced loads but buffeting loads to left hand side of the equations, the bridge deck response under a gusty wind can be estimated from:

$$
\begin{gathered}
M_{x} \ddot{x}+\left(G_{x}+\rho \bar{V} B C_{D}\right) \dot{x}+K_{x} x+\left[1 / 2 \rho \bar{V} B \frac{d C_{D}}{d \alpha} \dot{z}-1 / 2 \rho \bar{V}^{2} B \frac{d C_{D}}{d \alpha} \theta\right]= \\
\frac{\rho \bar{V} B}{2}\left[2 C_{D} u+\frac{d C_{D}}{d \alpha} \omega\right] \\
M_{z} \ddot{z}+\left(G_{z}+1 / 2 \rho \bar{V} B \frac{d C_{L}}{d \alpha}\right) \dot{z}+K_{z} z+ \\
{\left[\rho \bar{V} B C_{L} \dot{x}-1 / 2 \rho \bar{V}^{2} B \frac{d C_{L}}{d \alpha} \theta-1 / 2 \rho \bar{V} n B \dot{\theta}\right]=\frac{\rho \bar{V} B}{2}\left[2 C_{L} u+\frac{d C_{L}}{d \alpha} \omega\right]} \\
I_{\theta} \ddot{\theta}+\left(G_{\theta}+1 / 2 \rho B^{3} \frac{d C_{M}}{d \alpha} n \bar{V}\right) \dot{\theta}+\left(K_{\theta}-1 / 2 \rho \bar{V}^{2} B^{2} \frac{d C_{M}}{d \alpha}\right) \theta+\left[\rho \bar{V} B^{2} C_{M} \dot{x}+\right. \\
\left.1 / 2 \rho \bar{V} B^{2} \frac{d C_{M}}{d \alpha} \dot{z}\right]=\frac{\rho \bar{V} B^{2}}{2}\left[2 C_{M} u+\frac{d C_{M}}{d \alpha} \omega\right]
\end{gathered}
$$

where, $x, z$ and $\theta$ are the bridge responses in the horizontal, vertical and torsional directions, single and double dotted parameters are the first and second derivatives of the bridge responses with respect to the time, $M_{x}, M_{z}$ and $I_{\theta}$ are the effective inertias of the 
deck for horizontal, vertical and torsional motions, $G_{x}, G_{z}$ and $G_{\theta}$ are the effective viscous damping constants in the three directions, and $K_{x}, K_{z}$ and $K_{\theta}$ are the effective stiffnesses in the three directions.

From Eqs. (8) to (10) and Table 1, It can be seen that the steady force coefficients play an important role on the aerodynamic damping, aerodynamic stiffness and buffeting loads (Larose, 2002). Keeping in mind that the aerodynamic stiffness is often negligible compared to the structural stiffness and the fact that coupling terms are often small, the aerodynamic damping remains as the most important wind induced effect (Larose, 2002). For bridges at low wind angles of attack, $C_{D}$ is generally a positive value that can lead to a positive aerodynamic damping in the horizontal direction (see eq. (8)). However, based on the eq. (9), the vertical aerodynamic damping involves the factor $\frac{d C_{L}}{d \alpha}$ and can reduce the total vertical damping when this factor is negative. As a result, there is a potential for galloping at a certain wind speed when a section experiences negative $\frac{d C_{L}}{d \alpha}$. Eqs. (8) to (10) also show that the fluctuating loads due to the turbulence in the approaching wind are also dependent on the steady force coefficients and their derivatives with respect to the angle of attack.

The quasi steady assumption does not consider the effect of the out of phase components of aerodynamic forces that can also contribute to the aerodynamic damping and stiffness of the bridge. The out of phase components result from the structural motion and can be obtained from testing a sectional model in a dynamic test rig with at least two degrees of freedom in the heaving and torsional directions. 
Based on the quasi steady theory, the buffeting loads on a bridge deck can be calculated from the spectral approach which is widely used in the bridge aerodynamics field. The superiority of this method to time domain approaches is that with using this method, memory effects and self-excited forces can be explained reliably in the frequency domain. Memory effect is a term used to show that the bridge excitation at each time step $(t)$ depends on the interaction of the flow and the cross section at a time instant prior to that time step $(t-z)$. The memory effect is described in the frequency domain using an aerodynamic admittance function. Self-exited loads in vertical and torsional degrees of freedom (DOFs) can be computed from the following equations in frequency domain (Scanlan, 1978):

$$
\begin{aligned}
L_{h} & =\frac{1}{2} \rho \bar{V}^{2} B\left[K H_{1}{ }^{*}(K) \frac{\dot{z}}{\bar{V}}+K H_{2}{ }^{*}(K) \frac{B \dot{\theta}}{\bar{V}}+K^{2} H_{3}{ }^{*}(K) \theta+K^{2} H_{4}{ }^{*} \frac{z}{B}\right] \\
M_{\alpha} & =\frac{1}{2} \rho \bar{V}^{2} B^{2}\left[K A_{1}{ }^{*}(K) \frac{\dot{z}}{\bar{V}}+K A_{2}{ }^{*}(K) \frac{B \dot{\theta}}{\bar{V}}+K^{2} A_{3}{ }^{*}(K) \theta+K^{2} A_{4}{ }^{*} \frac{z}{B}\right]
\end{aligned}
$$

where, $K$ is the reduced frequency $(2 \pi f B / U), f$ is the frequency of motion $(\mathrm{Hz})$, $H_{i}{ }^{*}$ and $A_{i}{ }^{*}(i=1$ to 4$)$ are flutter derivatives. These equations, unlike the quasi steady formulations, consider the effect of the out of phase components of aerodynamic forces.

From the quasi steady assumption, wind-induced loads at each moment can be appreciated as loads generated by a steady wind having the same relative wind speed and direction as the momentary wind. The buffeting loads can be calculated from the following equations, as previously shown in Table 1:

$$
\begin{aligned}
& F_{L, b}=1 / 2 \rho \bar{V} B\left(2 C_{L} u+d C_{L} / d \alpha \omega\right) \\
& M_{b}=1 / 2 \rho \bar{V} B^{2}\left(2 C_{M} u+d C_{M} / d \alpha \omega\right)
\end{aligned}
$$


where $F_{L, b}$, and $M_{b}$ are the buffeting loads in the vertical and torsional directions per unit length of the span. In the spectral approach, it is assumed that buffeting loading is a stationary random process. As a result, eq. (13) can be transformed into eq. (14) in the frequency domain:

$$
S_{L}(f)=(1 / 2 \rho \bar{V} B)^{2}\left(4 C_{L}{ }^{2} S_{u}(f)|A(f)|^{2}+\left(d C_{L} / d \alpha\right)^{2} S_{\omega}(f)|A(f)|^{2}\right)
$$

Where $|A(f)|^{2}$ is the aerodynamic admittance function, and $S_{L}, S_{u}$ and $S_{\omega}$ are the power spectral densities of the lift per unit length, the wind fluctuations in the along-wind direction and wind fluctuations in the vertical direction, respectively. The application of the aerodynamic admittance function was suggested by Davenport (1962) to resolve some of the quasi-steady assumption limitations by including the effect lack of spatial correlation in the higher frequency turbulence fluctuations. Larose et al (1998) showed the difference between the aerodynamic admittance function obtained for a single box girder bridge from direct measurement and a thin airfoil from empirical equations using Sears function. It was shown that the admittance function calculated from the Sears function was larger than the measured admittance, particularly at lower frequencies. The aerodynamic admittance function for the vertical direction can be calculated from the direct measurement using the following equation:

$$
|A(f)|^{2}=\frac{\bar{V}^{2} S_{C_{L}}(f)}{4 C_{L}{ }^{2} S_{u}(f)+\left({ }^{d C_{L} / d \alpha}\right)^{2} S_{\omega}(f)}
$$

Eq. (15) gives the spectrum of the lift per unit length of the deck. In order to consider the span-wise distribution of the unsteady wind forces acting on the deck, the joint acceptance function is defined to transform point-like load to load on the entire span: 


$$
S_{F_{L}}(f)=S_{L}(f)\left|J_{z}(f)\right|^{2}
$$

where $S_{F_{L}}$ is the power spectral density of the lift effective on the entire span and $\left|J_{z}(f)\right|^{2}$ is the joint acceptance function. Joint acceptance function measures the correlation between the spatial distributions of the forces across the span length. Based on the strip assumption, wind-induced loads on each strip are resulted only from the wind fluctuations on that strip. Therefore, it is assumed that spatial distribution of the windinduced loads across the span length follows the wind spatial distribution. However, Larose et al (1993) showed that wind generated loads could be more correlated than the approaching wind, between any two assumed points across the span.

\subsection{Objectives}

Wind flow characteristics and deck geometry affect the wind structure interaction. As a result, in order to capture the response of a prototype from experiments, it is important to satisfy the similarity requirements as far as possible. Matching atmospheric conditions in a wind tunnel requires matching Re, turbulence intensities, integral scales, spectral characteristics of the velocity fluctuations, etc. To the author's knowledge there are not any studies in the literature which take into account the combined effects of turbulence parameters and $R e$ on the aerodynamic and aeroelastic behavior of long span bridges, in particular vortex induced vibration. The main objective of this research is to evaluate the effects of $R e$ and turbulence characteristics on the aerodynamic response of a twin deck bridge using a motionless model. Also, the effect of vortex generators on the vortex shedding was tested at different $R e$ regimes to validate the efficacy of these devices on vortex mitigation at higher $R e$ which is comparable to the prototype condition. 
There are some studies in the literature considering the effect of bridge deck shape and detailing on the vortex induced vibration of long span bridges (Larsen et al, 2008). However, few of them considered these effects in a properly simulated wind flow with high Re and flow turbulence parameters. One factor which appears to have significant effect on the aerodynamic/aeroelastic response of twin deck bridges is the gap width. Also, the literature lacks conclusive statements about the effect of deck features such as traffic barriers on the bridge response. The second objective of this research is to study the effects of aforementioned properties on the aerodynamic response of twin deck bridges.

The last objective of this dissertation is to provide detailed requirements for developing a large scale testing facility to perform dynamic sectional model tests at higher $R e$ compared to conventional wind tunnel testing. The design approach for an experimental setup which is capable of performing free vibration sectional model testing in the Wall of Wind (WOW) open jet facility at Florida International University (FIU) is thus provided.

In order to depict the effect of the aforementioned parameters (Re, turbulence characteristics and shape details) on the aerodynamic response of a bridge deck, several parameters were investigated. The first parameter to study was the pressure distribution around the girders. It is well documented that the pressure distribution can give an insight on the flow separation and reattachment zones and therefore can be used to predict the source of excitation mechanisms (Kwok et al, 2012; Li and Melbourne, 1995). The second type of parameter to study included the static force/moment coefficients and their derivatives with respect to angle of attack. As described earlier, these parameters can 
describe some of the aerodynamic features of bridges based on quasi steady theory. The last parameter to study was the lift spectrum. This can be used to evaluate vortex shedding and buffeting loads.

The findings from this research can be useful for engineers, designing twin box girder bridges, by informing them on the potential effects of the given parameters, and provide guidance for laboratory similitude requirements for twin box girder bridge sections. It also provides valuable information on how changing the gap width can change the aerodynamic performance of twin girder bridges and what could be the excitation mechanism behind it so that designers can adopt the best gap width to satisfy aerodynamic requirements.

\subsection{Dissertation Organization}

This dissertation is written in the format of 'Thesis Containing Journal Papers'. The dissertation contains three manuscripts for scholarly journals, of which one is published and two will be submitted in the near future. In addition, one chapter is added to address the development of a dynamic test rig which can be used in the WOW to guide section model testing of large scaled models in future. Also, a general introduction chapter is provided at the beginning and a general conclusion chapter is given at the end of the dissertation.

The general introduction chapter consists of a brief introduction on the topic, explanation of different wind experiments on bridges, theoretical background, objectives and finally a summary of the main content of the following chapters.

The first paper, published in the "Journal of Wind and Structures", investigates the effects of Reynolds number on the aerodynamic characteristics of a twin-deck bridge. 
Static tests were performed on a 1:36 scale sectional model of a twin girder bridge, instrumented with pressure taps and load cells, at high wind speeds with $R e$ ranging from $1.3 \times 10^{6}$ to $6.1 \times 10^{6}$ based on the section horizontal width. The results presented are based on the measured data from the described model at the Wall of Wind open jet wind tunnel facility at Florida International University. Results are presented in the form of pressure distributions, force/moment coefficients and Strouhal numbers for the different tested $R e$ regimes. Also, the effect of $R e$ on the load distribution between the two girders is discussed. Finally, the efficiency of vortex mitigation devices is compared for the different tested $R e$ regimes to evaluate the $R e$ sensitivity of such devices.

The second paper, going to be submitted to the "Journal of Wind Engineering and Industrial Aerodynamics, discusses the sensitivity of the aerodynamic behavior of a twin deck bridge cross-section to the incoming flow turbulence characteristics. Chord-wise pressure measurements were carried out on the motionless $1 / 36$ and $1 / 72$ scaled sectional models of the bridge under two flow conditions using the WOW facility at FIU. The results are given in terms of pressure distribution, force coefficients, lift spectra (to study vortex shedding), and aerodynamic admittance and span-wise correlation of windinduced loads (to study buffeting wind loadings). The results given in this section can help designers with information on the potential effects of turbulence characteristics on instability, vortex shedding and buffeting loads.

The third paper, going to be submitted to the "Journal of Wind and Structures", investigates the effect of deck details, such as gap width, traffic barriers and bike path on the aerodynamic characteristics of a twin deck bridge, particularly on the vortex shedding. In order to evaluate the effect of gap width, models with three different gap 
widths were tested in a static condition. The effect of deck furniture was also studied by including the traffic barriers, bike path and appendages in the simulation for the smallest gap width. In all the tests, the models were equipped with pressure taps that were distributed along the chord length. The wind induced loads were also measured directly by load cells. The outcomes of this paper benefit the design in several ways. First of all, it clarifies the vortex shedding mechanism for the different gap widths so that the optimum gap width can be adopted to reduce the adverse effects of vortex shedding. Also, from quasi steady assumption, the effect of gap width on some of the aerodynamic features such as galloping can be evaluated. The results regarding modeling the traffic barriers and bike path can also shed light on the importance of modeling such devices in the wind experiments and to evaluate how this modeling can interfere with the aerodynamic response of the model.

The last chapter is a general guideline and gives general information on the system designed and built for the sake of this study so that it can be used later on in WOW to study other types of structures and for different applications. The designed test rig can be used to study a wide range of aerodynamic effects including vortex induced vibration, flutter and buffeting on 2-D models of prismatic structures such as bridges, light poles and etc. The novelty of this test setup is that it can be used to study large models in high wind speeds so that the testing Reynolds number is closer to the prototype range than in most other facilities. Testing at a larger scale is also beneficial to modeling more detailing so that the geometry is closer to the prototype. This system enables simultaneous vertical and torsional motions of the vibrating model and therefore it can be used to evaluate aeroelastic effects on numerous structures. Vibration frequencies of the 
system can be tuned with changing the number of springs and the spring stiffness. It is also possible to use this test rig for performing static test on the model by changing the setup slightly. In that case, only the interaction of the wind and section is considered without taking into account the self-excited forces generated due to structural motion.

\subsection{References}

Wind Directionality Factors for Nonhurricane and Hurricane-Prone Regions. Journal of Structural Engineering 0, 04014208.

Blevins, R.D., 2001. Flow-induced Vibration. Krieger Publishing Company.

Chen, X., Kareem, A., 2002. Advances in Modeling of Aerodynamic Forces on Bridge Decks. Journal of Engineering Mechanics 128, 1193-1205.

Chowdhury, A.G., Sarkar, P.P., 2003. A new technique for identification of eighteen flutter derivatives using a three-degree-of-freedom section model. Engineering Structures $25,1763-1772$.

Davenport, A.G., 1961. The spectrum of horizontal gustiness near the ground in high winds. Quaterly Journal of the Royal Meteorological Society 87.

Davenport, A.G., 1962. Buffeting of a suspension bridge by storm winds. Journal of Structural Engineering 88, 233-270.

Davenport, A.G., Isyumov, N., Miyata, T., 1971. The Experimental Determination of the Response of Suspension Bridges to Turbulent Wind Third International Conference on Wind Effects on Buildings and Structures, Tokyo, Japan.

Frandsen, J.B., 2001. Simultaneous pressures and accelerations measured full-scale on the Great Belt East suspension bridge. Journal of Wind Engineering and Industrial Aerodynamics 89, 95-129.

Fransos, D., Bruno, L., 2010. Edge degree-of-sharpness and free-stream turbulence scale effects on the aerodynamics of a bridge deck. Journal of Wind Engineering and Industrial Aerodynamics 98, 661-671.

Fujino, Y., Yoshida, Y., 2002. Wind-Induced Vibration and Control of Trans-Tokyo Bay Crossing Bridge. Journal of Structural Engineering 128, 1012-1025.

Ge, Y.J., Xiang, H.F., 2008. Bluff body aerodynamics application in challenging bridge span length, 6th International Colloquium on Bluff Bodies Aerodynamics and Applications, Milano,Italy. 
Gu, M., Zhang, R., Xiang, H., 2000. Identification of flutter derivatives of bridge decks. Journal of Wind Engineering and Industrial Aerodynamics 84, 151-162.

Haan, F., Kareem, A., 2009. Anatomy of Turbulence Effects on the Aerodynamics of an Oscillating Prism. Journal of Engineering Mechanics 135, 987-999.

Haan Jr., F.L., Kareem, A., Szewczyk, A.A., 1998. The effects of turbulence on the pressure distribution around a rectangular prism. Journal of Wind Engineering and Industrial Aerodynamics 77-78, 381-392.

Hsia, T.C., 1976. On least squares algorithms for system parameter identification. Automatic Control, IEEE Transactions on 21, 104-108.

Hui, M.C.H., Larsen, A., 2002. Aerodynamic Investigations for the Deck of the Stonecutters Bridge Emphasizing Reynolds Number Effects in: Proceedings of the The Second International Symposium on Wind and Structures, 649-656.

Imai, H., Yun, C.B., Maruyama, O., Shinozuka, M., 1989. Fundamentals of system identification in structural dynamics. Probabilistic Engineering Mechanics 4, 162-173.

Irwin, P.A., 1977. Wind Tunnel and Analytical Investigations of the Response of Lions Gate Bridge To Turbulent Wind. National Research Council of Canada, National Research Council of Canada, p. 62.

Irwin, P.A., 1998. The role of wind tunnel modeling in the prediction of wind effects on bridges, in: Esdahl, A.L.S. (Ed.), The international symposium on advances in bridge aerodynamics, Copenhagen, Denmark, pp. 99-117.

Iwamoto, M., Fujino, Y., 1995. Identification of flutter derivatives of bridge deck from free vibration data. Journal of Wind Engineering and Industrial Aerodynamics 54-55, 5563.

Jones, N.P., Scanlan, R.H., Sarkar, P.P., Singha, L., 1995. The effect of section model details on aeroelastic parameters. Journal of Wind Engineering and Industrial Aerodynamics 54-55, 45-53.

K. Matsuda, K.R.C., H. Tanaka, M. Tokushige, T. Iwasaki, 2001. An investigation of Reynolds number effects on the steady and unsteady aerodynamic forces on a 1:10 scale bridge deck section model. Journal of Wind Engineering and Industrial Aerodynamics $89,619-632$.

Kargarmoakhar, R., Chowdhury, A.G., Irwin, P.A., 2015. Reynolds Number Effects on Twin Box Girder Long Span Bridge Aerodynamics. Wind and Structures.

Kwok, K.C.S., Qin, X.R., Fok, C.H., Hitchcock, P.A., 2012. Wind-induced pressures around a sectional twin-deck bridge model: Effects of gap-width on the aerodynamic 
forces and vortex shedding mechanisms. Journal of Wind Engineering and Industrial Aerodynamics 110, 50-61.

Laima, S., Li, H., Chen, W., Li, F., 2013. Investigation and control of vortex-induced vibration of twin box girders. Journal of Fluids and Structures 39, 205-221.

Larose, G.L., 2002. The dynamic action of gusty winds on long-span bridges, Department of Structural Engineering and Materials. Technical University of Denmark, p. 179.

Larose, G.L., D'Auteuil, A., 2006. On the Reynolds number sensitivity of the aerodynamics of bluff bodies with sharp edges. Journal of Wind Engineering and Industrial Aerodynamics 94, 365-376.

Larose, G.L., Davenport, A.G., King, J.P.C., 1993. On the Unsteady Aerodynamic Forces on a Bridge Deck in Turbulent Boundary Layer Flow, 7th U.S. National Conference on Wind Engineering, California, USA.

Larose, G.L., Tanaka, H., Gimsing, N.J., Dyrbye, C., 1998. Direct measurements of budeting wind forces on bridge decks. Journal of Wind Engineering and Industrial Aerodynamics 74-76, 809-818.

Larsen, A., Esdahl, S., Andersen, J.E., Vejrum, T., 2000. Storebælt suspension bridge vortex shedding excitation and mitigation by guide vanes. Journal of Wind Engineering and Industrial Aerodynamics 88, 283-296.

Larsen, A., Savage, M., Lafrenière, A., Hui, M.C.H., Larsen, S.V., 2008. Investigation of vortex response of a twin box bridge section at high and low Reynolds numbers. Journal of Wind Engineering and Industrial Aerodynamics 96, 934-944.

Larsen, A., Wall, A., 2012. Shaping of bridge box girders to avoid vortex shedding response. Journal of Wind Engineering and Industrial Aerodynamics 104-106, 159-165.

Li, Q.S., Melbourne, W.H., 1995. An experimental investigation of the effects of freestream turbulence on streamwise surface pressures in separated and reattaching flows. Journal of Wind Engineering and Industrial Aerodynamics 54-55, 313-323.

Lin, Y.-Y., Cheng, C.-M., Lan, C.-Y., 2003. Effects of deck's width-to-depth ratios and turbulent flows on the aerodynamic behaviors of long-span briges. Wind and Structures 6, 263-278.

Liu, H., 1991. Wind Engineering: A Handbook for Structural Engineers. Prentice Hall.

Ma, R., Chen, A., 2007. Determination of flutter derivatives by a taut strip model. Journal of Wind Engineering and Industrial Aerodynamics 95, 1400-1414. 
Macdonald, J.H.G., Irwin, P.A., Fletcher, M.S., 2002. Vortex-induced vibrations of the Second Severn Crossing cable-stayed bridge\&\#8212;full-scale and wind tunnel measurements, Proceedings of the ICE - Structures and Buildings, pp. 123-134.

Neuhaus, C., Höffer, R., 2011. Identification of quasi-stationary aeroelastic force coefficients for bridge deck sections using forced vibration wind tunnel testing, in: Roeck, G.D., Degrande, G., Lombaert, G., Muller, G. (Eds.), 8th International Conference on Structural Dynamics, EURODYN 2011, Leuven, Belgium, pp. 13861392.

Sarkar, P.P., Jones, N.P., Scanlan, R.H., 1992. System identification for estimation of flutter derivatives. Journal of Wind Engineering and Industrial Aerodynamics 42, 12431254.

Sato, H., Hirahara, N., Fumoto, K., Hirano, S., Kusuhara, S., 2002. Full aeroelastic model test of a super long-span bridge with slotted box girder. Journal of Wind Engineering and Industrial Aerodynamics 90, 2023-2032.

Scanlan, R.H., 1978. The action of flexible bridges under wind, I: Flutter theory Journal of Sound and Vibration 60, 187-199.

Scanlan, R.H., Jones, N.P., Lorendeaux, O., 1997. Comparison of taut-strip and sectionmodel-based approaches in long-span bridge aerodynamics. Journal of Wind Engineering and Industrial Aerodynamics 72, 275-287.

Scanlan, R.H., Tomko, J.J., 1971. Airfoil and Bridge Deck Flutter Derivatives. Journal of the Engineering Mechanics Division 97, 1717-1737.

Schewe, G., 2001. Reynolds number effects in flow around a more-or-less bluff bodies. Journal of Wind Engineering an Industrial Aerodynamics 89, 1267-1289.

Schewe, G., Larsen, A., 1998. Reynolds number effects in the flow around a bluff bridge deck cross section. Journal of Wind Engineering and Industrial Aerodynamics 74-76, 829-838.

Simiu, E., Scanlan, R.H., 1996. Winds Effects on Structures: Fundamentals and Applications to Design. John Wiley \& Sons, New York.

Theodorsen, T., 1935. General theory of aerodynamic instability and the mechanism of flutter, Technical Report no. 496, NACA, Technical Report no. 496

pp. $413-433$.

Wardlaw, R.L., Tanaka, H., Utsunomiya, H., 1983. Wind tunnel experiments on the effects of turbulence on the aerodynamic behaviour of bridge road decks. Journal of Wind Engineering and Industrial Aerodynamics 14, 247-257. 
Zhu, L.D., Xu, Y.L., Xiang, H.F., 2002. Tsing Ma bridge deck under skew winds-Part II: flutter derivatives. Journal of Wind Engineering and Industrial Aerodynamics 90, 807837. 
CHAPTER II

REYNOLDS NUMBER EFFECTS ON TWIN BOX GIRDER LONG SPAN BRIDGE AERODYNAMICS

(A paper published in the journal of Wind and Structures) 


\title{
CHAPTER II
}

REYNOLDS NUMBER EFFECTS ON TWIN BOX GIRDER LONG SPAN BRIDGE

\section{AERODYNAMICS}

\author{
Ramtin Kargarmoakhar ${ }^{\mathrm{a}}$, *Arindam Gan Chowdhury ${ }^{\mathrm{b}}$, Peter Irwin ${ }^{\mathrm{c}}$
}

\subsection{Abstract}

This paper investigates the effects of Reynolds number $(R e)$ on the aerodynamic characteristics of a twin-deck bridge. A 1:36 scale sectional model of a twin girder bridge was tested using the Wall of Wind (WOW) open jet wind tunnel facility at Florida International University (FIU). Static tests were performed on the model, instrumented with pressure taps and load cells, at high wind speeds with $R e$ ranging from $1.3 \times 10^{6}$ to $6.1 \times 10^{6}$ based on the section width. Results show that the section was almost insensitive to $R e$ when pitched to negative angles of attack. However, mean and fluctuating pressure distributions changed noticeably for zero and positive wind angles of attack while testing at different $R e$ regimes. The pressure results suggested that with the Re increase, a larger separation bubble formed on the bottom surface of the upstream girder accompanied with a narrower wake region. As a result, drag coefficient decreased mildly and negative lift coefficient increased. Flow modification due to the $R e$ increase also helped in distributing forces more equally between the two girders. The bare deck

${ }^{a} \mathrm{PhD}$ candidate, Civil and Environmental Engineering/International Hurricane Research Center, Florida International University, Miami, FL, USA, rkarg001@fiu.edu

b Corresponding Author, Associate Professor, Civil and Environmental Engineering/International Hurricane Research Center, Florida International University, Miami, FL, USA, chowdhur@fiu.edu, Tel: (305) 348-0518

${ }^{c}$ Professor of Practice, Civil and Environmental Engineering/International Hurricane Research Center, Florida International University, Miami, FL, USA, peairwi@fiu.edu 
section was found to be prone to vortex shedding with limited dependence on the $R e$. Based on the observations, vortex mitigation devices attached to the bottom surface were effective in inhibiting vortex shedding, particularly at lower $R e$ values.

Keywords: Reynolds number effect; twin-box girder bridge; vortex shedding; force and moment coefficient; pressure Distribution; aerodynamic response.

\subsection{Introduction}

In order to estimate wind loads for structural design of bridges and to predict the aerodynamic performance of the bridge cross section, usually wind tunnel tests are carried out on scaled models of the structure. To ensure the proper simulation of the aerodynamic/aeroelastic response of the structure in a wind tunnel, similitude of certain non-dimensional parameters is necessary. One such non-dimensional parameter, Reynolds number, represents the ratio of inertial force to viscous force in a fluid. Conventional small-scale tests are often performed at $R e$ that is lower than its full-scale counterpart by a factor of about $10^{2}$ to $10^{3}$, thus violating the $R e$ similitude by a large margin. This is largely for practical reasons since matching Reynolds number is not possible in conventional wind tunnels using small models and low wind speed. Testing at lower Re compared to the prototype is often considered to be representative of the aerodynamic loading and responses of the full-scale structure for sharp edged bluff bodies, such as buildings and bridges. This is due to the assumption that the separation point of the flow is fixed on a bridge or other bluff structures with sharp edges and they can have aerodynamic characteristics almost insensitive to $R e$ as long as a $R e$ of greater than 10,000 is reached (Larose and D'Auteuil, 2006). However, several researches including some more recent literature (Asghari Mooneghi et al, 2014; Barre and Barnaud, 
1993; Kazutoshi et al, 2007; Larose and D’Auteuil, 2006; Larose et al, 2003; Larsen et al, 2008; Schewe and Larsen, 1998), show that wind loads and the response of bluff structures with sharp edges -- particularly bridges -- can be sensitive to $R e$ effects. It was also shown that $R e$ increase can reduce the imbalances of aerodynamic forces between the two girders for twin girder bridges (Lee et al, 2014).

The aerodynamic performance of any structure immersed in a fluid depends on different parameters, such as $R e$ and turbulence characteristics, which can affect the flow separation and reattachment mechanism, laminar to turbulent boundary layer transition, and the behavior of shear layers and separation bubbles. It is known that Reynolds number can change the location of the laminar to turbulent transition point which subsequently modifies the structure of the wake (Schewe and Larsen, 1998). Schewe (2001) tested three different bluff structures to explain the reason behind the $R e$ sensitivity of the drag coefficient and Strouhal Number $(S t)$. It was noticed that with the $R e$ increase, the location of laminar to turbulent transition point moved upstream and helped the flow to reattach to the section, leaving a smaller wake. Smaller drag force and smaller vortices, being shed at a higher frequency, resulted from the change in the location of transition point and a smaller wake region. It was concluded that different flow regimes can be distinguished based on the $R e$ that could affect the $S t$ and magnitude of steady and unsteady forces (Schewe, 2001). In order to minimize the $R e$ effects on the aerodynamic behavior of bridges, Lee et al (2014) successfully fixed the location of the separation point at the bottom of the deck by attaching a boundary layer trip strip to the bottom surface. This is similar to a movable pin that Schewe (2001) added to a circular 
cylinder section that reduced the $R e$ dependency through stabilizing the location of the transition point.

One typical aeroelastic phenomenon which causes concerns in the design of modern multiple girder bridges is Vortex Induced Vibration (VIV) (Wu and Kareem, 2012). VIV occurs when the frequency of the vortices shed from the body approaches the modal frequency of the bluff body, creating a resonance type motion in the structure. While VIV is mostly a limited amplitude vibration that does not lead to a failure directly, it can yield to fatigue damage in bridge members and discomfort for drivers. Larsen et al (2008) performed free vibration testing on a model of the Stonecutters bridge, a twin girder bridge with mildly curved bottom plates, for a range of $R e$ from $0.7 \times 10^{5}$ to $3.62 \times 10^{5}$, based on the individual girder width. While the velocity range and the amplitudes of vibration corresponding to the VIV were almost insensitive to the $R e$ variation for the bare section, the modified section with the guide vanes showed significant $R e$ sensitivity. In order to mitigate or more desirably to avoid vortex induced oscillations in bridges, evaluation of the $R e$ effect can be important in so far as it can change both the Strouhal number (St) and the amplitude of vibrations during the lock-in phenomenon (Kazutoshi et al, 2007; Kubo et al, 1999; Larose and D'Auteuil, 2006; Larose et al, 2012). The $R e$ effect on flutter instability was investigated by K. Matsuda (2001) and it turned out that testing at higher $R e$ inclined to increase the flutter wind speed for the given configurations.

The prototype $R e$ for bridges often ranges between $10^{6}$ and $10^{7}$, especially for high wind events such as thunderstorms and tropical cyclones. Studies on $R e$ effects pertaining to twin girder bridges, a type of design that is gaining popularity in recent 
years, are limited. In view of the potential for $R e$ effects on wind loads and wind-induced dynamic effects on twin box girder long span bridges, the current research was aimed at investigating the aerodynamics of such bridges under a range of $R e$ as close as possible to its prototype counterpart. The key objective of this research was to investigate the effect of $R e$ on the aerodynamic characteristics of twin girder bridges, including pressure distribution, wind induced forces, Strouhal number, and vortex shedding. In order to investigate the effects of $R e$ on the bridge response, a 1:36 scale model of a two dimensional (2-D) section of a twin girder bridge, based on the section of the "East Span of the San Francisco-Oakland Bay Bridge" was constructed and tested using the Wall of Wind (WOW) open jet wind tunnel facility at Florida International University (FIU). High Reynolds numbers were reached by increasing the testing wind speed up to 48.2 $\mathrm{m} / \mathrm{s}$. Forces and pressures were measured using load cells and pressure taps to capture the static and fluctuating forces on the deck due to the approach flow. Vortex shedding sensitivity to $R e$ was evaluated for both the bare deck configuration and a section equipped with vortex generators. The efficacy of the vortex generators on mitigating vortex shedding was investigated for different $R e$ regimes. The research findings can (1) help inform engineers designing double box girder bridges on the potential $R e$ effects, and (2) provide guidance for laboratory $R e$ similitude for twin box girder bridge sections. The present study is different from those documented in the literature in the following aspects:

- The cross section studied here was a double girder deck with trapezoidal cross section for each girder which has sharper edges and is bluffer compared to the more streamlined sections studied before. 
- Vortex generators and guide vanes represent different approaches to vortex shedding mitigation. Previously, only the effects of guide vanes on vortex shedding were evaluated for different $R e$ regimes. In this study, efficacy of vortex generators in mitigating vortex shedding has been evaluated for different $R e$ regimes.

\subsection{Experimental Setup}

\subsubsection{Twin box girder bridge deck}

In this study, the $R e$ effects on the static force coefficients and fluctuating surface pressures for a twin box deck bridge were evaluated. To this end, the bare cross sectional shape of the eastern span of the San Francisco-Oakland bridge (East Bay Bridge) deck with the inclusion of the traffic barriers was selected and tested in a high Reynolds number regime ranging from $1.3 \times 10^{6}$ to $6.1 \times 10^{6}$. The East span of the San FranciscoOakland bay bridge is an asymmetric self-anchored suspension bridge with a main span of $385 \mathrm{~m}$ and back span of $185 \mathrm{~m}$ (Fig. 1). This bridge features a twin deck configuration carried by self-anchored suspension cables where the two decks are linked together with $10 \mathrm{~m}$ wide transverse beams every $20 \mathrm{~m}$. The single deck chord length $(C)$, total width $(B)$ and height $(H)$ of the bridge deck are $28 \mathrm{~m}, 71 \mathrm{~m}$ and $5.5 \mathrm{~m}$, respectively (Fig. 1). Each girder resembles a trapezoidal cross section shape with aspect ratio close to the approach span of the Great Belt east bridge for which noticeable $R e$ effects were observed (Schewe and Larsen, 1998). The bike path that exists on the real bridge was not included in the test setup as it would have added to the complexity of maintaining model rigidity at high wind speeds. In the current study, $R e$ effects on the efficacy of vortex generator devices for the mitigation of vortex shedding were also investigated. These devices are used for the real bridge and represent diverging pairs of vertical rectangular 
plates attached to the bottom surfaces of both girders as shown in Fig. 1. As the name suggests, each pair of vortex generators is meant to facilitate the formation of a pair of counter rotating vortices which are intended to promote reattachment of the flow to the bridge surface. The functionality of these vertical plates is to inject a strong high speed flow into the gap between the decks, thus inhibiting the periodic vortex shedding and mitigating vortex induced vibrations in the prototype bridge structure. The information on the bridge specifications was provided by T.Y. LIN INTERNATIONAL GROUP, which was involved in all phases of developing this bridge, from designing to construction.

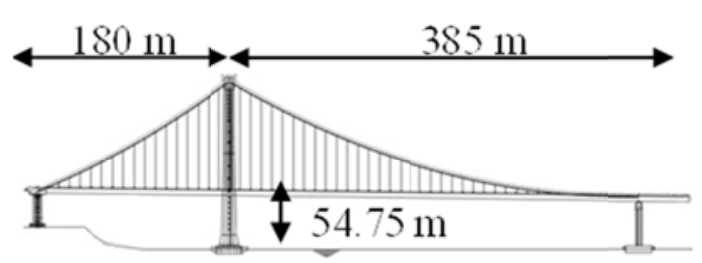

(a) General layout

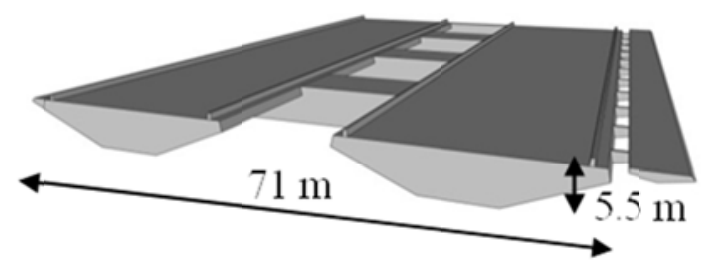

(b) Cross sectional shape

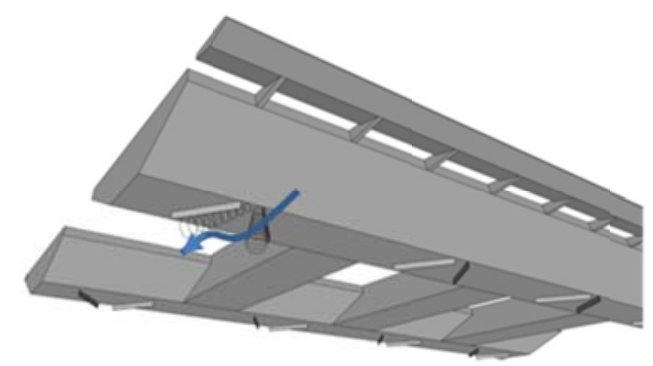

(c) Vortex generators on the bottom surface

Figure 1: East span of the San Francisco-Oakland bay bridge

\subsubsection{Measurement instrument and test setup}

For this study, a rigid pressure-tapped sectional model of the twin-deck bridge was tested using the 12-fan WOW at FIU. A length scale of 1:36 was used to simulate the aerodynamic geometry of the bridge deck. A sectional view of the bridge section model 
and the distribution of the pressure taps are shown in Fig. 2. The maximum span length of the model was $3 \mathrm{~m}$. Two large streamlined end walls covered the two ends of the model to reduce the end effects and provide a 2-D simulation of the wind flow. The rigid model was made out of a wooden frame and was covered by Plexiglas plates to produce the cross-section shape. The model was equipped with five strips of pressure taps in order to measure fluctuating surface pressure distributions. One strip was located in the middle of the span and the other strips were located as shown in Fig. 3 to evaluate the span-wise distribution of pressures. Pressures were measured at a total of 280 points with a 512 channel Scanivalve Corporation pressure scanning system at sampling frequency of $512 \mathrm{~Hz}$ for a period of two minutes for each test case. Pressure scanners were placed at both ends of the model to reduce the pressure tube length. A transfer function designed for the tubing was used to correct for the tubing effects (Irwin et al, 1979).

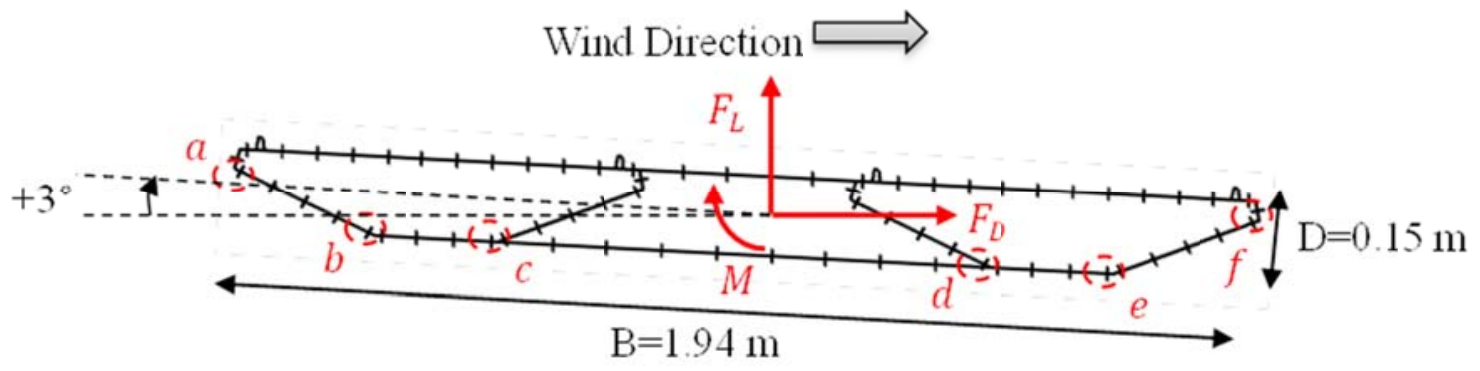

Figure 2: Positions of the pressure taps (model is tilted to $+3^{\circ}$ angle of attack a) Leading edge, $b$ ) Windward tipping edge of the upstream girder, $c$ ) Leeward tipping edge of the upstream girder, $d$ ) Windward tipping edge of the downstream girder, $e$ ) Leeward tipping edge of the downstream girder, $f$ ) Trailing edge 


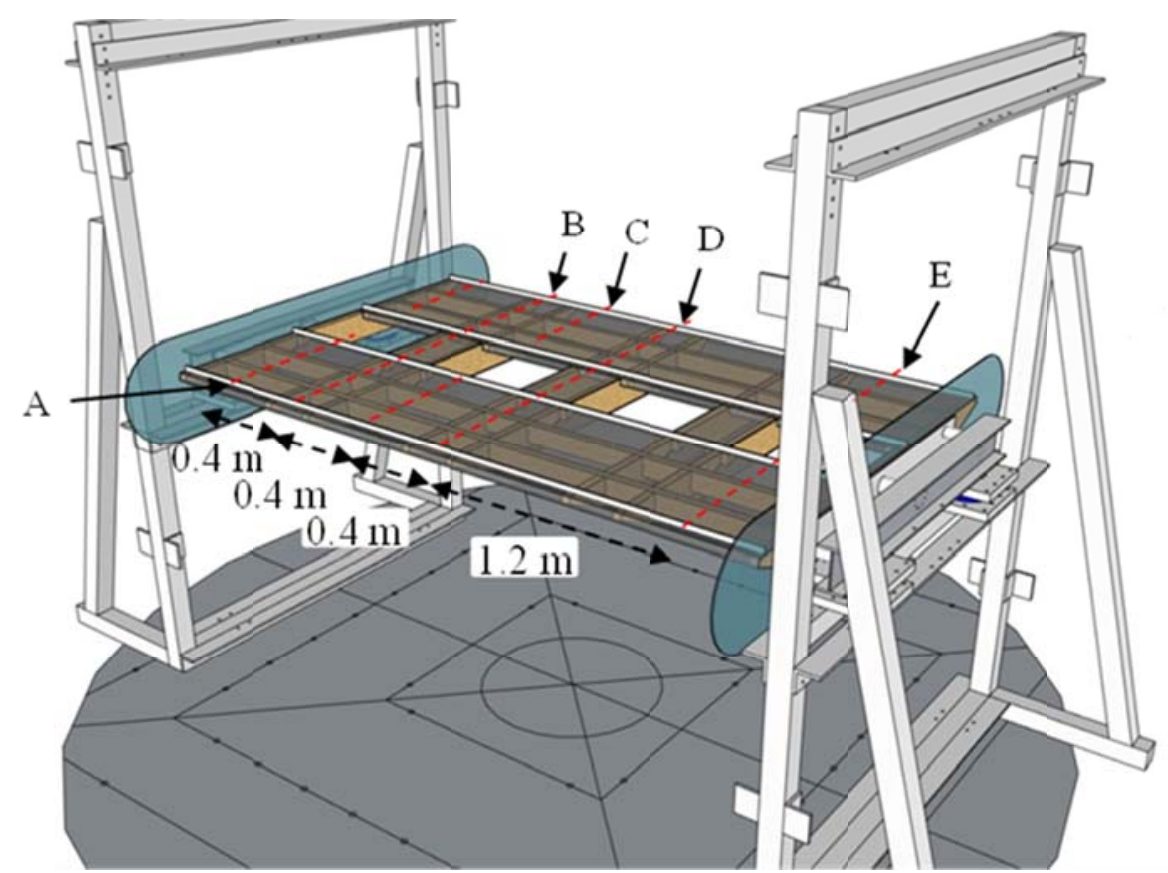

Figure 3: Testing setup, "A" to "E" show the distribution of the chord-wise pressure tapped strips along the span length

Considering the width of the model to be equal to $1.94 \mathrm{~m}$, the span to width ratio of the section model was 1.6. This ratio is similar to the ratio used in a previous research by Laima et al (2013) to study the VIV of twin-girder bridges. Also, Piña and Caracoglia (2009) studied flutter derivatives for the Carquinez Strait Bridge using two models with different span to width ratios and suggested that span to width ratio of 1.7 was adequate for 2-D experiments.

The model for the current study was installed on an aluminum I-beam on each end using three aluminum pipe elements to allow for the simulation of various wind angles of attack by adjusting the pitch of the model (see Fig. 3). Five wind angles of attack were tested including $-6^{\circ},-3^{\circ}, 0^{\circ},+3^{\circ}$, and $+6^{\circ}$. Positive angle was defined as the one which was measured from the horizontal to the axis of the bridge as shown in Fig.2. Each beam was mounted on a pair of JR3 multi-axis load cells to measure the forces (Drag and Lift) 
and the pitching moment (see Fig. 3) simultaneously with the pressure measurements. All the data from force scanners were recorded using a CompactRIO data acquisition system at $100 \mathrm{~Hz}$ sampling rate. Both systems, load cells and pressure scanners, were used to facilitate comparison of wind effects.

\subsubsection{Flow characteristics}

The 12-fan WOW open jet facility at FIU was used to generate the wind field for the present study. WOW is capable of simulating Atmospheric Boundary Layer (ABL) mean wind speed profiles and turbulence characteristics. The nominal cross sectional dimensions of the testing section are $4.6 \mathrm{~m} \times 6.1 \mathrm{~m}$. Wind tunnel tests on bridges are often performed in flows with minimal turbulence as such flow produces conservative results. Gu et al (2001) observed that flutter derivatives of the Jiangyin Bridge and a flat plate with aspect ratio of 22.5 were almost insensitive to the approaching flow turbulence. High turbulence can increase the diffusion of vortices and as a result reduce the vortex shedding strength (Wu and Kareem, 2012). As a result, high turbulence has been found to be conducive for disrupting vortex induced vibration in bridges (Wardlaw et al, 1983). Thus, for the current study a flow condition with minimum turbulence was generated by removing the spires and floor roughness elements that are used in WOW for generation of ABL turbulence for testing building models. Figure 4 shows a close-up view of the experimental apparatus in its final design, during the execution of the tests for the zero angle of attack. 


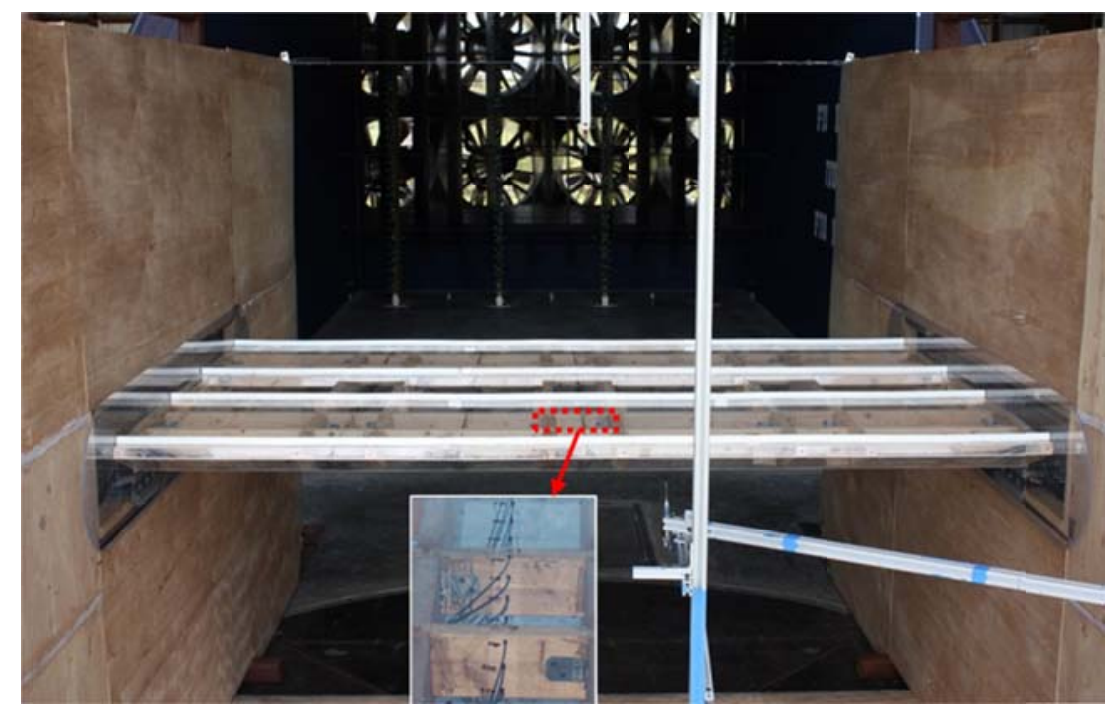

Figure 4: 1:36 scale model in front of Wall of Wind, Florida International University

Wind speeds were measured both during the tests on the bridge model and for free flow wind speed measurements (the bridge model was removed).

In order to measure the wind speeds during each test, two cobra probes were placed at $0.9 \mathrm{~m}$ below and above the middle point of the section to minimize the local effects of the model on the measured wind speed. The average of the measured wind speeds at these two points was used as the mean wind speed in the calculation of the pressure, force and moment coefficients.

For the free wind speed measurement, five cobra probes were used to measure the wind speeds (see Fig 5). Two probes were installed at the same locations at which wind speeds were measured during the tests on models (probes 1 and 2). The other three cobra probes were installed at the bridge height, one at the center and the other two at equidistance between the center and the end walls (probes 3, 4, and 5). The ratio of the averaged wind speed of the two points at $0.9 \mathrm{~m}$ distant (probes 1 and 2) to the spatial average of measured wind speeds at the bridge height (average of probes 3, 4 and 5) was 
calculated for the free flow condition (i.e. $r=U_{\operatorname{avg} \text { of probes } 1 \text { and2 } 2} / U_{\text {avg of probes 3,4,and5). }}$ ). The pressure, force and moment coefficients results were adjusted to the bridge height wind speed by multiplying each coefficient to $r^{2}$.

Data measured from the Cobra probes were collected at $100 \mathrm{~Hz}$ using the same CompactRIO data acquisition system that was used for force measurements. Tests were carried out for a range of wind speeds from 10 to $48.2 \mathrm{~m} / \mathrm{s}$, simulating $R e$ values (based on the model width) between a range of $1.3 \times 10^{6}$ to $6.1 \times 10^{6}$. The $R e$ for the prototype bridge can be estimated as $5.9 \times 10^{7}$ for wind speeds of $12.5 \mathrm{~m} / \mathrm{s}$, corresponding to the VIV lock-in wind speed for the first vertical mode of vibration at $0.214 \mathrm{~Hz}$ frequency. Based on the wind speed and turbulence measurements, the turbulence intensity and turbulence integral length scale were estimated as 3.0 percent and $0.2 \mathrm{~m}$ at the model height, respectively. Figure 6 shows the non-dimensional power spectral density (PSD) of the longitudinal component of the turbulent wind speed, measured at the model height. In order to get a smooth representation of the spectra, averaging of the spectral analysis of 11 blocks was performed and presented here (each block was formed of 1024 points). Table 1 shows a summary of the tests carried out in this study. 


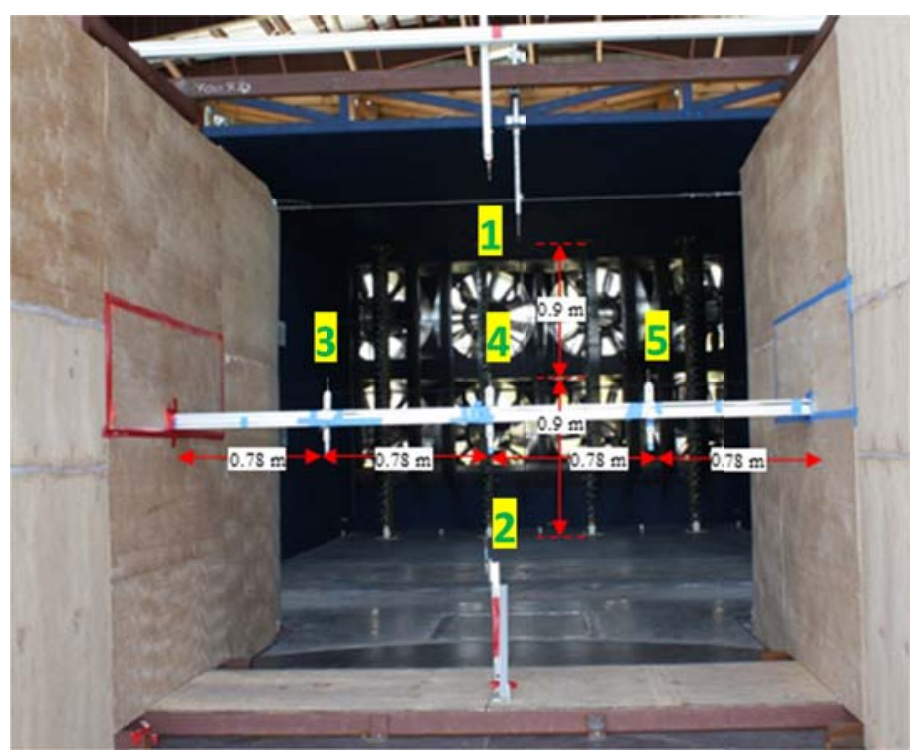

Figure 5: Cobra Probes Position

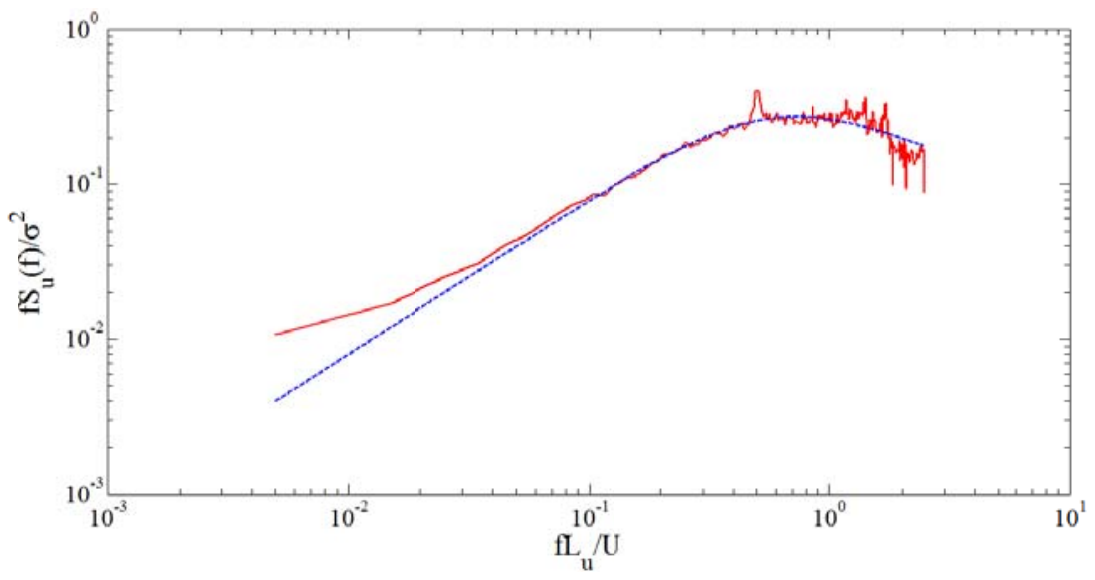

Figure 6: Normalized power spectral density (PSD) of the longitudinal component of the wind speed at bridge height

Karman Spectrum — Simulated Spectrum

Table 1: Test Summary

\begin{tabular}{|c|c|c|c|}
\hline Test case & Angle of attack & Wind speed $(\mathrm{m} / \mathrm{s})$ & Test duration (min) \\
\hline Bare section & $-6^{\circ},-3^{\circ}, 0^{\circ},+3^{\circ},+6^{\circ}$ & $\begin{array}{r}10.0-13.3-20.3-24- \\
27.3-34.6-41.2-48.2\end{array}$ & 2 \\
\hline $\begin{array}{c}\text { Section fitted with } \\
\text { vortex generators }\end{array}$ & $-6^{\circ},-3^{\circ}, 0^{\circ},+3^{\circ},+6^{\circ}$ & $\begin{array}{r}10.0-13.3-20.3-24- \\
27.3-34.6-41.2-48.2\end{array}$ & 2 \\
\hline
\end{tabular}




\subsection{Parameters Investigated for $R e$ Effects}

At high Reynolds number, inertia forces dominate over the fluid viscous forces. At low $R e$, viscous forces have an increased effect on the aerodynamics. $R e$ is dependent both on the model size and testing wind speed and is expressed as:

$$
R e=\frac{U B}{v}
$$

where, $U$ is mean wind speed $(\mathrm{m} / \mathrm{s}), B$ is the reference dimension (in this case the deck width (m)), $v$ is kinematic viscosity of air $\left(\mathrm{m}^{2} / \mathrm{s}\right)$.

For the case of constant kinematic viscosity, higher $R e$ can be achieved only either by increasing the size of the model or the wind speed or both. The sensitivity of the following parameters to the $R e$ was studied in the current work.

\subsubsection{Pressure coefficients}

Mean and fluctuating pressure distributions can be used to identify the regions of flow separation and reattachment in the studies of bluff body aerodynamics (Kwok et al, 2012). In this study, mean and fluctuating pressure distributions were considered in order to highlight the changes in the structure of the flow due to the $R e$ variation. Pressure distributions are reported in terms of non-dimensional mean and rms (root mean square) pressure coefficients $\left(C_{\bar{P}}\right.$ and $C_{\sigma_{P}}$, respectively) obtained as:

$$
C_{\bar{P}}=\frac{P_{\text {avg }}}{1 / 2_{2} \rho U^{2}}, C_{\sigma_{P}}=\frac{\sigma_{P}}{1 / 2^{\rho} \rho U^{2}}
$$

where: $P_{a v g}$ is the mean pressure $\left(\mathrm{N} / \mathrm{m}^{2}\right)$ obtained from the pressure time history data at each tap, $\sigma_{P}$ is the standard deviation of pressure time history at each tap $\left(\mathrm{N} / \mathrm{m}^{2}\right)$, $\rho$ is the air density $\left(\mathrm{kg} / \mathrm{m}^{3}\right)$, and $U$ is the mean wind speed $(\mathrm{m} / \mathrm{s})$ at the model height. 


\subsubsection{Aerodynamic forces}

Aerodynamic forces acting on the bridge deck can be expressed in terms of the two force components of Drag $\left(F_{D}\right)$ and Lift $\left(F_{L}\right)$ and the pitching Moment $(M)$. As shown in Fig. 2, Drag is defined as the force component acting parallel to the wind direction, Lift is the force component perpendicular to the wind direction and pitching Moment is defined as the effective moment about the deck geometric center. $C_{D}, C_{L}$ and $C_{M}$ are the normalized form of the aerodynamic force/moment obtained, using the dynamic pressure and model dimensions, as:

$$
C_{D}=\frac{F_{D}}{\frac{1}{2} \rho U^{2} L H}, C_{L}=\frac{F_{L}}{\frac{1}{2} \rho U^{2} L B}, C_{M}=\frac{M}{\frac{1}{2} \rho U^{2} L B^{2}}
$$

where, $F_{D}$ is the mean drag $(\mathrm{N}), F_{L}$ is the mean lift $(\mathrm{N}), M$ is the mean pitching $(\mathrm{N} \cdot \mathrm{m}), \rho$ is the air density $\left(\mathrm{kg} / \mathrm{m}^{3}\right), U$ is the mean wind speed $(\mathrm{m} / \mathrm{s})$, and $B, H$ and $L$ represent the deck chord, deck height (i.e. vertical depth) and the length of the model, respectively (m).

\subsubsection{Self-excited forces}

Self-exited forces induced by wind for a bridge deck, with vertical and torsional degrees of freedom (DOFs), are obtained from the following equations (Scanlan, 1978):

$$
\begin{aligned}
& L_{h}=\frac{1}{2} \rho U^{2} B\left[K H_{1}{ }^{*}(K) \frac{\dot{h}}{U}+K H_{2}{ }^{*}(K) \frac{B \dot{\alpha}}{U}+K^{2} H_{3}{ }^{*}(K) \alpha+K^{2} H_{4}{ }^{*} \frac{h}{B}\right] \\
& M_{\alpha}=\frac{1}{2} \rho U^{2} B^{2}\left[K A_{1}{ }^{*}(K) \frac{\dot{h}}{U}+K A_{2}{ }^{*}(K) \frac{B \dot{\alpha}}{U}+K^{2} A_{3}{ }^{*}(K) \alpha+K^{2} A_{4}{ }^{*} \frac{h}{B}\right]
\end{aligned}
$$

where, $K$ is the reduced frequency $(2 \pi f B / U), f$ is the frequency of motion $(\mathrm{Hz})$, $h$ and $\alpha$ are the vertical and torsional displacements, over-dot indicates the derivatives with respect to time and $H_{i}{ }^{*}$ and $A_{i}{ }^{*}(i=1$ to 4$)$ are flutter derivatives. 
Memory effects cause aerodynamic forces to be influenced by structural motions. Therefore, since quasi steady theory ignores such effects it is at best an approximation. However, it has been shown that for high reduced velocities, quasi steady theory can nonetheless predict aerodynamic forces associated with lateral and vertical motions reasonably well (Chen and Kareem, 2002). It has been also attempted to define derivatives corresponding to the torsional motion $\left(\mathrm{A}_{2}{ }^{*}\right.$ and $\left.\mathrm{H}_{2}{ }^{*}\right)$ from the quasi steady theory (Chen and Kareem, 2002; Larose and Livesey, 1997; Neuhaus and Höffer, 2011). All of these studies related the $A_{2}{ }^{*}$ parameter to $d C_{M} / d \alpha$.

However, an additional parameter was introduced in all cases which represented the eccentricity between the point of application of the generated aeroelastic force and the wind flow's idealized point of incidence. It was shown that this parameter was not constant for different force components and different shapes (Chen and Kareem, 2002; Neuhaus and Höffer, 2011) which can cause error in the correct estimation of $A_{2}{ }^{*}$ and $H_{2}{ }^{*}$ using quasi steady methods. In addition, Schewe (2009) stated that the onset wind speed of torsional galloping is proportional to the inverse of $d C_{M} / d \alpha$.

The quasi-steady formulation for $H_{1}{ }^{*}$ is given by (Chowdhury and Sarkar, 2004):

$$
H_{1}^{*}=-\frac{1}{K}\left(\frac{d C_{L}}{d \alpha}+C_{D}\right)
$$

where, $d C_{L} / d \alpha$ is the derivative of lift coefficient with respect to the angle of attack.

\subsubsection{Vortex shedding}

When wind blows over a bluff structure, flow separates and causes shedding of vortices periodically. This periodic vortex shedding exerts cross-wind forces on the body by creating fluctuating pressures. Strouhal number is a non-dimensional parameter that 
defines the dominant frequency of the fluctuations in the cross-wind force and is expressed as:

$$
S t=\frac{f H}{U}
$$

where, $f$ is the frequency of vortex shedding $(\mathrm{Hz}), H$ is the bridge depth (m); $U$ is the oncoming wind speed $(\mathrm{m} / \mathrm{s})$. St is a function of structure's geometry, turbulence intensity and $R e$. As stated earlier, vortex induced vibration (VIV) occurs when the frequency of vortex shedding approaches the natural frequency of the bluff body. The vortex shedding frequency can be obtained to a first approximation from the power spectral density (PSD) of the fluctuating lift force on the section. The peak evident in the fluctuating lift spectrum shows the frequency of the vortex shedding (Kwok et al, 2012; Schewe and Larsen, 1998).

\subsection{Experimental Results and Discussion}

\subsubsection{Re effects on stream-wise pressure distribution}

Figures 7, 8 and 9 show the distribution of mean pressures and rms of fluctuating pressures around the bare deck cross section. Negative mean pressures are shown outside of the bridge deck and positive mean pressures are shown inside of the bridge deck. Large (in magnitude) negative local pressures indicate the flow separation region. Fluctuating pressures represent the turbulent flow around the section which can be caused either by the intrinsic wind turbulence or the body induced turbulence (signature turbulence).

For the negative angles of attack, separation regions are noticed behind the traffic barrier on the top surface of both girders and at windward tipping edge at the lower 
surface of the upstream girder (see Fig. 7(a) and 7(b)). The fluctuating pressure distribution indicates that the downstream girder was immersed in the wake flow of the upstream girder (see Fig. 9(a) and 9(b)). This cross section was almost insensitive to the $R e$ variation (for the $R e$ interval tested here) when it was tilted to negative angles. This might be due to the fact that, traffic barriers fixed the location of the flow separation point on the top surface and therefore eliminated the $R e$ sensitivity.

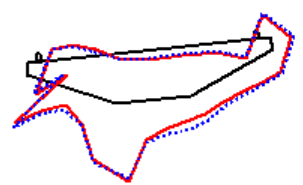

(a) Angle of attack $=-6^{\circ}$

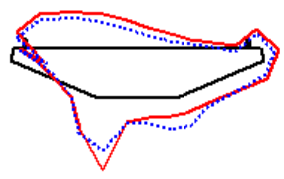

(c) Angle of attack $=0^{\circ}$
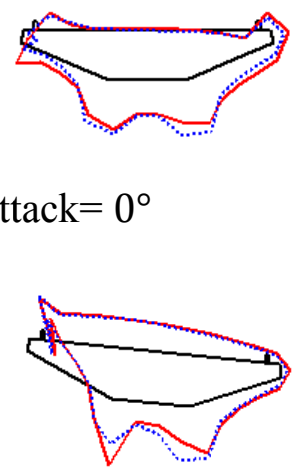

(e) Angle of attack $=+6^{\circ}$

Figure 7: Mean pressure distribution

$-1.27 \times 10^{6} \cdots \cdots \cdots \cdot 6.13 \times 10^{6},\left(C_{\bar{p}}=1: \longmapsto\right)$




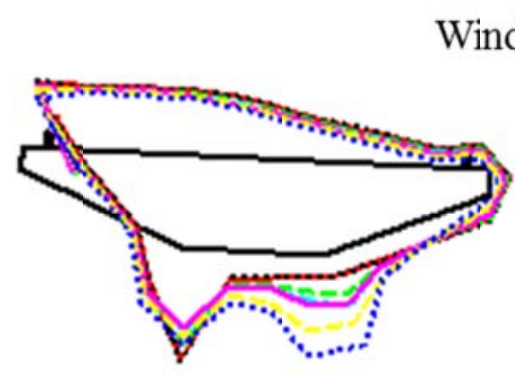

irection

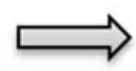

Figure 8: Mean pressure distribution at $+3^{\circ}$ wind angle of attack

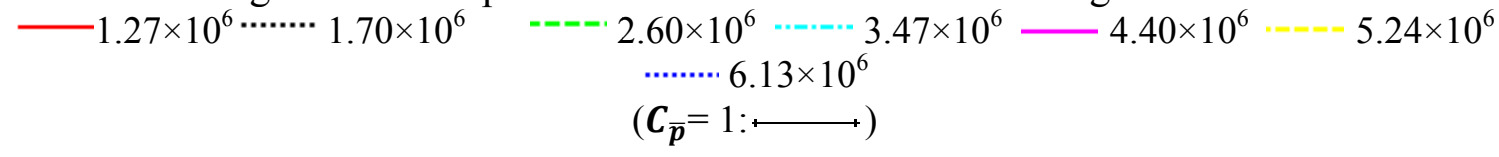

Wind
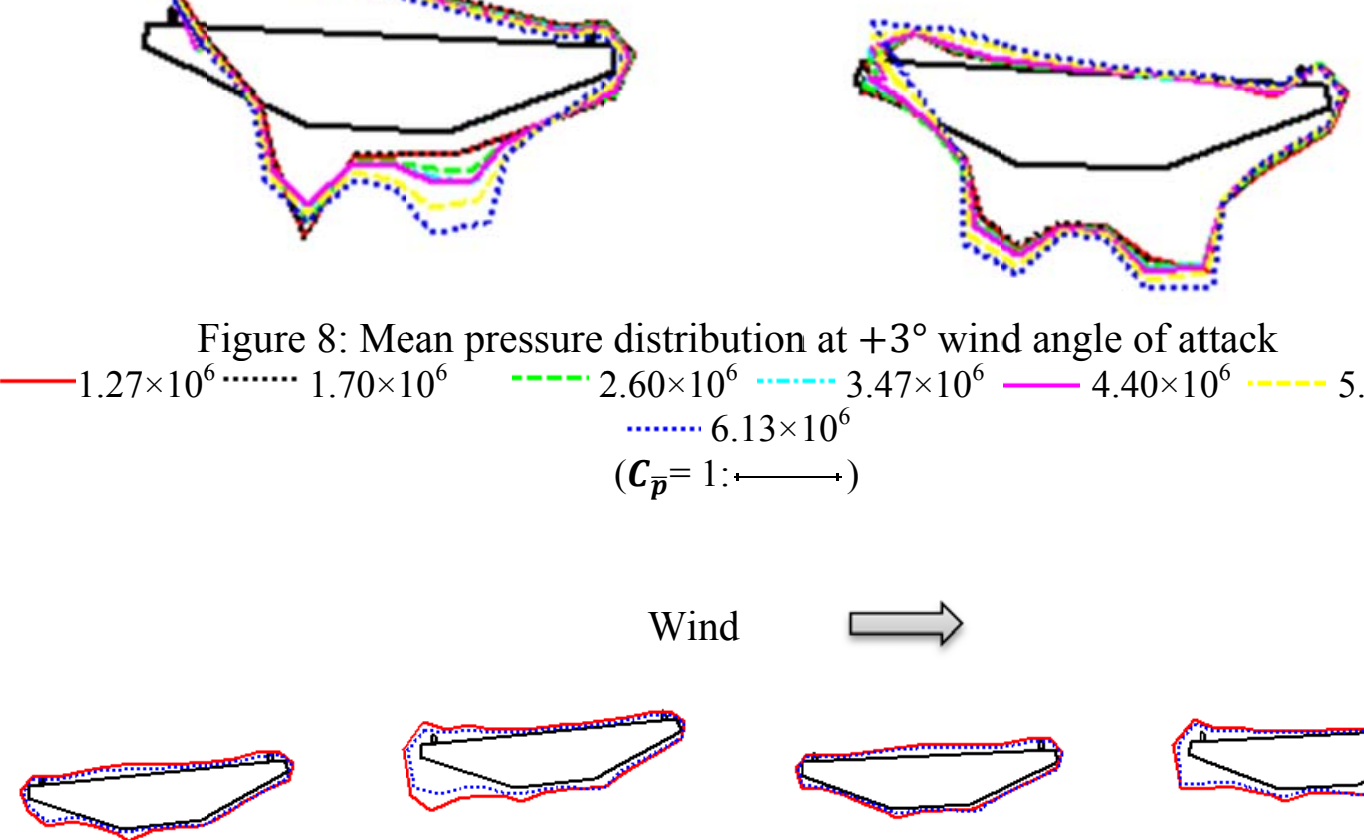

(a) Angle of attack $=-6^{\circ}$
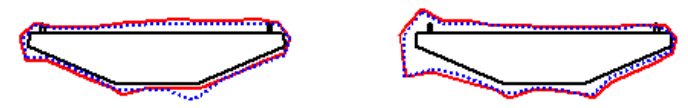

(c) Angle of attack $=0^{\circ}$
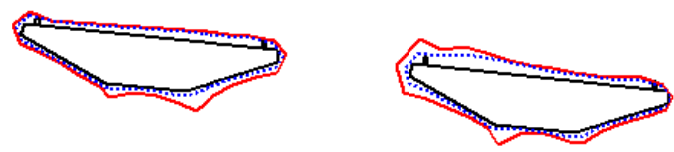

(e) Angle of attack $=+6^{\circ}$

Figure 9: rms of Fluctuating Pressure Distribution

$$
\begin{gathered}
-1.27 \times 10^{6} \ldots \ldots \ldots .6 .13 \times 10^{6} \\
\left(\boldsymbol{C}_{\sigma_{p}}=0.5: \longleftrightarrow\right)
\end{gathered}
$$

For the zero wind angle of attack, Figs. 7(c) and 9(c) indicate flow separation at the traffic barrier location on the windward side of the upstream girder which created a turbulent flow across the top surface of both girders with no clear evidence of reattachment. The turbulence in the flow over the top surfaces is recognized from the 
appreciable magnitude of the rms of the fluctuating pressure (see Fig. 9(c)). Based on Fig. 7(c), for the lower $R e$ regime, the flow was ventilated through the gap in between the two girders due to the pressure difference between the top and bottom surfaces. Therefore the ventilation of flow through the gap created negative pressures on the windward inclined surface of the downstream girder. As the Re increased, the separated flow at the windward tipping edge of the upstream girder tended to reattach to the surface, creating a smaller wake in the downstream of the upstream girder and consequently a smaller drag on the upstream girder. Negative pressures on the windward inclined surface of the downstream girder were reduced with the $R e$ increase, as the passing flow from the bottom of the upstream girder prevented the ventilation of the flow through the gap.

From Figs. 7(d) and 7(e), it can be concluded that the deck cross section was more sensitive to $R e$ variation for the positive angles of attack. Flow over the top surface followed a similar trend to the case of zero angle of attack. This means that the flow was separated at the windward traffic barrier on the upstream deck and did not reattach to the surface while showing minimal sensitivity to Re. However, as shown in Fig. 7(d) and 9(d), flow behavior changed with $R e$ significantly on the lower side, particularly for the upstream deck. Separated flow at the windward tipping edge of the upstream girder reattached to the surface over a longer length but with a sharper slope for the higher $R e$. This indicates that $R e$ increase created a larger separation bubble on the bottom surface accompanied with a narrower wake region in the after body for the upstream girder, creating larger negative lift but smaller drag. Figure 8 shows how the mean pressure distribution around the section at $+3^{\circ}$ wind angle of attack has changed gradually with the 
$R e$ increase. Similar but less pronounced behavior was noticed for the $+6^{\circ}$ wind angle of attack (see Fig. 7(e) and 9(e)).

\subsubsection{Re effects on force coefficients}

Figure 10 shows the variation of the mean drag, lift and moment coefficients due to the $R e$ change for the bare section at zero wind angle of attack, measured with the load cells. From pressure distribution in Fig. 7(c), it can be concluded that with the $R e$ increase, negative pressures on the downwind inclined surfaces of both girders decreased leading to a smaller drag. By increasing the $R e$, smaller separation zone formed on the top surface of the upstream girder that led to smaller negative pressures on this surface. With the $R e$ increase, the negative lift coefficient increased in magnitude due to the modification of the pressure distribution around the two girders, particularly over the top and bottom surfaces (see Figs. 7(c) and 10(b)).

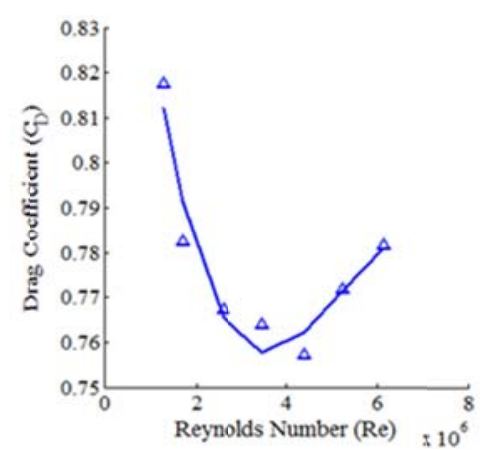

(a) Drag coefficient

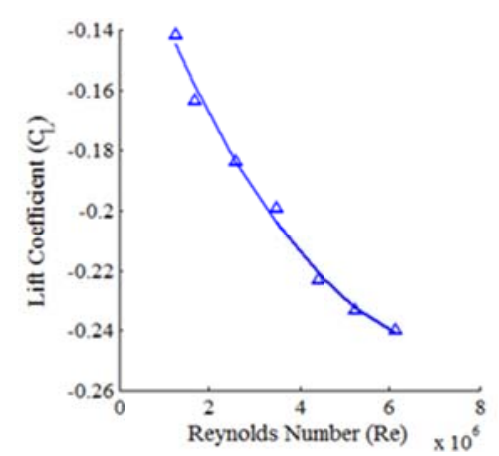

(b) Lift coefficient

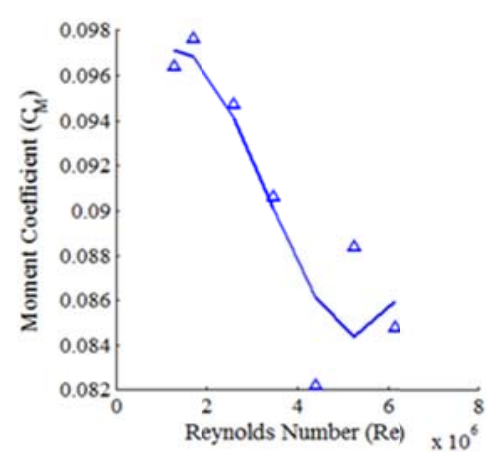

(c) Moment coefficient

Figure 10: Force coefficients as a function of Reynolds number at $0^{\circ}$ angle of attack $\triangle$ Load Cells

Figure 11 shows the variation of the aerodynamic force coefficients, obtained from the load cells, with the angles of attack and $R e$ for the bare section. Compared to the negative wind angles of attack, $R e$ sensitivity was more pronounced for positive angles of 
attack, just as was indicated by the pressure distribution results (see Sec. 4.1). As discussed in Section 2.5.1, the windward traffic barrier on the top surface of the upstream deck dictated the location of the separation point, thus eliminating the $R e$ sensitivity for the negative angles of attack. The drag coefficient showed a mild decreasing trend with the $R e$ increase. The maximum decrease in drag coefficient due to $R e$ variation was $24 \%$ for the positive 6 degree angle of attack. With the $R e$ increase, flow separation became more prevalent at the leeward tipping edge of the upstream deck while flow reattachment on the leeward side of both girders led to a smaller wake. Smaller wake and accordingly lower negative pressure on the leeward inclined surface of the girders reduced the drag force. The magnitude of the changes due to $R e$ in lift coefficients were in the order of 0.1 . A larger separation at higher $R e$ created a larger downward lift. The moment coefficient also showed some minor sensitivity to the $R e$ variation.

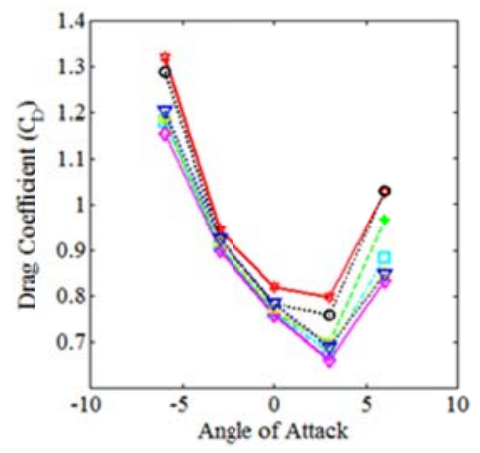

(a) Drag coefficient

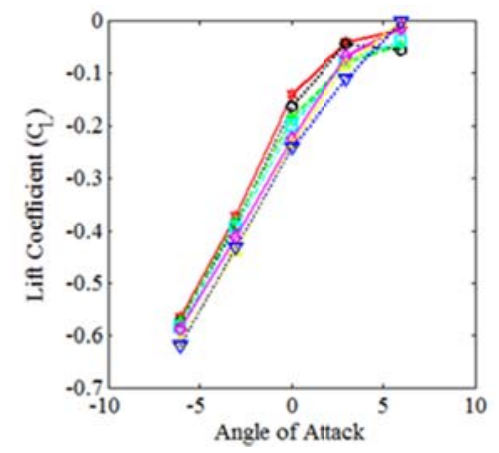

(b) Lift coefficient

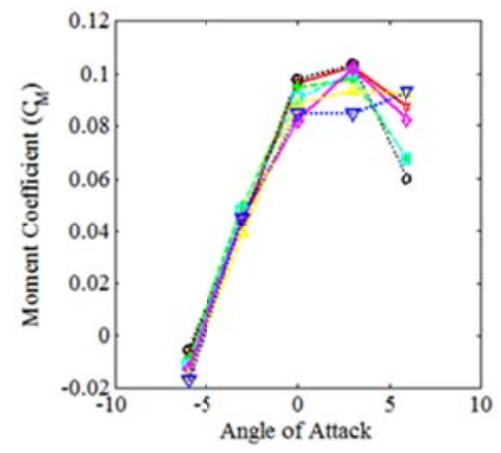

(c) Moment coefficient

Figure 11: Static aerodynamic coefficients as a function of wind angle of attack

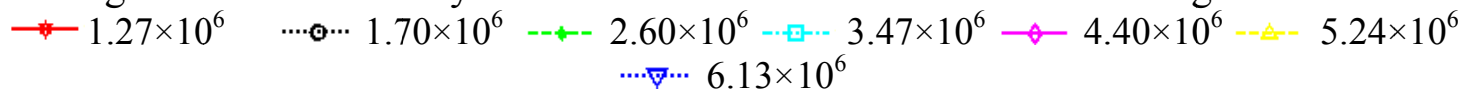

Figure 12 presents the derivative of force coefficients, obtained from load cells data, with respect to the angle of attack for the bare deck. Unlike $d C_{D} / d \alpha$ which showed little sensitivity to the $R e$ variation, $d C_{L} / d \alpha$ and $d C_{M} / d \alpha$ were more $R e$ sensitive, 
particularly for positive angles of attack. Based on quasi-steady theory, a negative value of $d C_{L} / d \alpha$ results in a positive value for $H_{1}{ }^{*}$ that shows a potential for vertical galloping. As shown in Fig. 12, negative $H_{1}{ }^{*}$ only happened for positive angles of attack (mainly for $+6^{\circ}$ ). Increasing the $R e$ changed the sign for $d C_{L} / d \alpha$ from negative to positive. This means that while testing at lower $R e$ regime can show aerodynamic instabilities, the section was more aerodynamically stable at higher $R e$ regimes. Kazutoshi et al (2007) also found that testing for flutter stability in a low $R e$ regime is conservative.

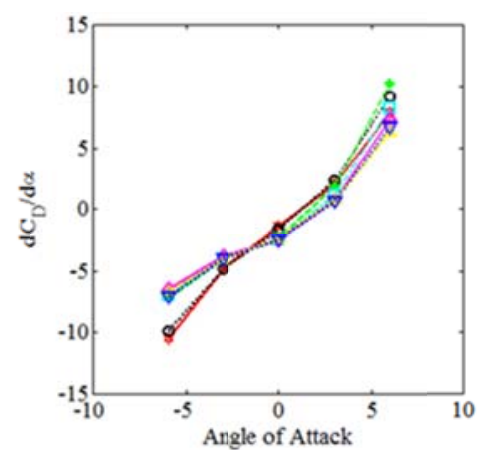

(a) $d C_{D} / d \alpha$

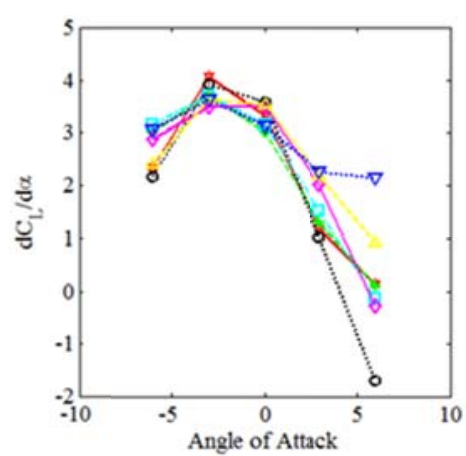

(b) $d C_{L} / d \alpha$

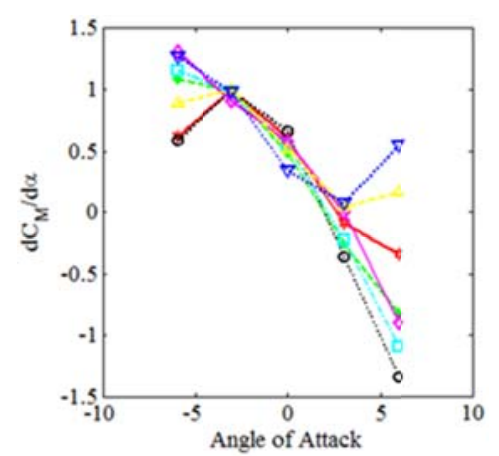

(c) $d C_{M} / d \alpha$

Figure 12: Derivative of force coefficients with respect to the angle of attack

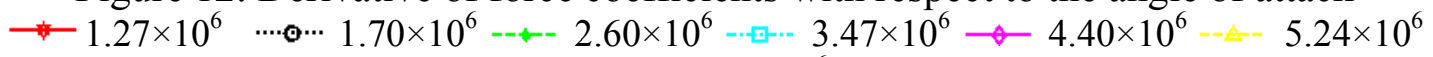
$\cdots \cdot \nabla \cdots \cdot 6.13 \times 10^{6}$

\subsubsection{Re effects on aerodynamic load sharing between the two girders}

Figures 13 and 14 show the variations of the ratio of the loading on each girder to the total effective force due to the $R e$. Each aerodynamic force component obtained from pressure measurement was considered as the total effective force on the deck and loading on each girder was estimated by integrating the mean pressures over a single girder. The results show some $R e$ dependence of the load shared by each girder. The drag force was mainly carried by the upstream girder. As shown in Fig. 7, negative pressure was formed on the upper surface of the upstream girder which balanced the negative pressures on the 
bottom surface, thus reducing the net downward lift. Such pressure equalization was not evident for the downstream girder (as shown in Fig. 7), which resulted in a significant downward lift for the downstream girder which shared most of the total lift.

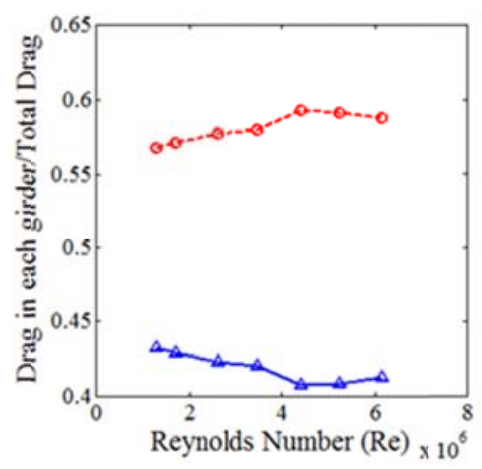

(a) Angle of attack $=-3^{\circ}$

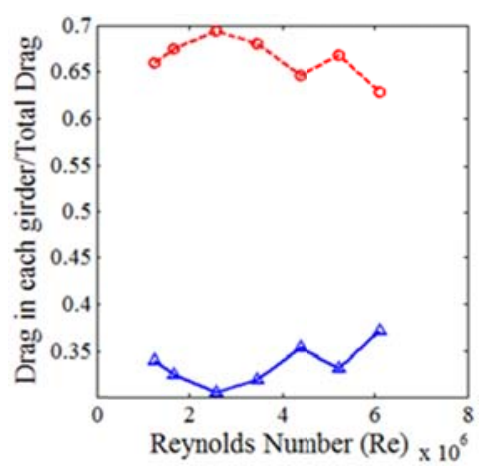

(b) Angle of attack $=0^{\circ}$

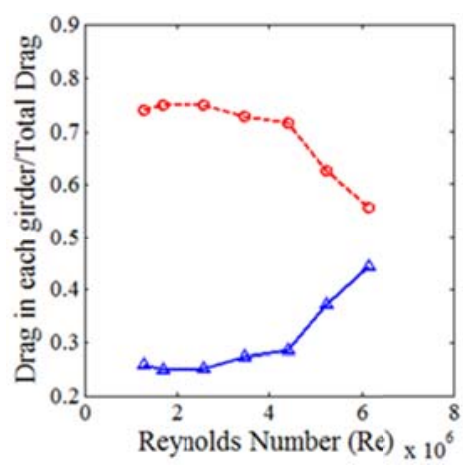

(c) Angle of attack $=+3^{\circ}$

Figure 13: Allotment of each girder from drag $--\theta-$ Upstream $\rightarrow$ Downstream

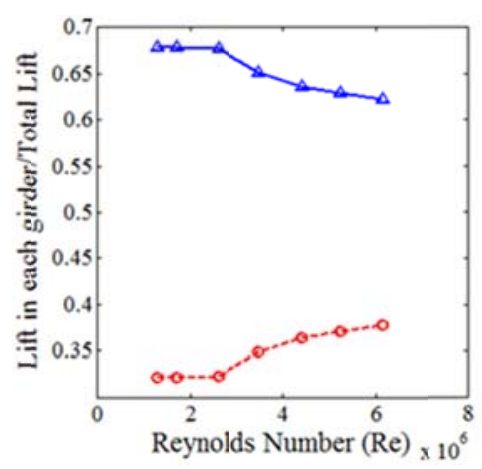

(a) Angle of attack $=-3^{\circ}$

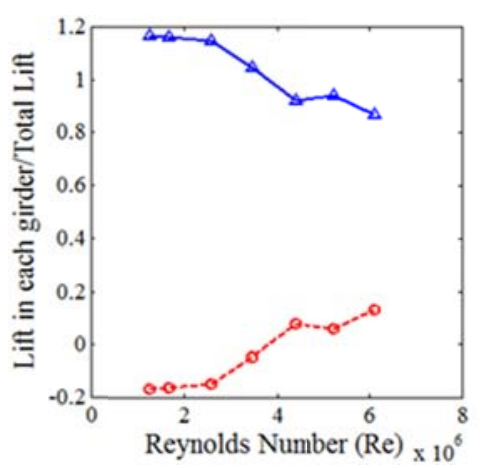

(b) Angle of attack $=0^{\circ}$

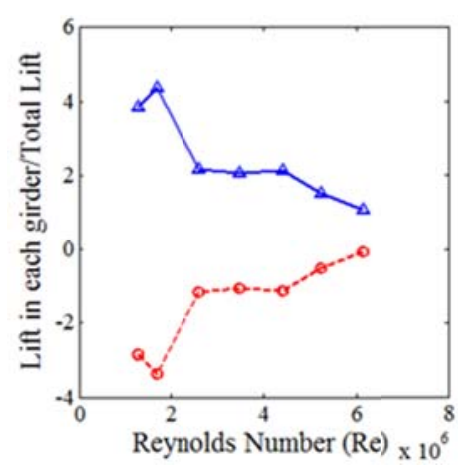

(c) Angle of attack $=+3^{\circ}$

Figure 14: Allotment of each girder from lift $--\bullet-$ Upstream $\rightarrow$ Downstream

Testing at higher $R e$ led to a more even distribution of loads between the two girders, with the exception of drag for $-3^{\circ}$ wind angle of attack. For this cross section, drag was mainly generated from the pressures on the inclined surfaces of the girders. For the higher $R e$, similar pressures formed on the inclined surfaces of the two girders. The 
ratio of the drag taken by each girder to the total drag was quite similar for the two girders which is in agreement with the results on drag presented in Lee et al (2014). Lee et al (2014) expressed that the sheltering effect is minimized with the $R e$ increase, resulting in more uniform load sharing between the two girders.

Figure 14 shows the ratio of the lift force in each girder to the total lift. Note that the total lift, as shown in Fig. 11, was a negative value (downward force) at all different wind angles of attack for the entire $R e$ range tested. It can be seen that the lift was not distributed equally between the two girders, particularly for the zero and positive wind angles of attack. It can be noticed that the upstream girder was under a positive (upward) lift for the zero and positive wind angles of attack at lower $R e$. This happened due to the large negative pressures caused by the traffic barrier at the leading edge of the upstream girder (see Fig. 7(c)-(d)). However, with the $R e$ increase, pressure distribution changed around the girders and as a result a downward lift was formed on the upstream girder at higher $R e$.

\subsubsection{Re effects on vortex shedding}

Figure 15 shows the lift coefficient power spectral density against the reduced frequency for the bare deck at the maximum and minimum tested wind speeds and for $6^{\circ}, 0^{\circ}$ and $+6^{\circ}$ wind angles of attack. Similar plots were obtained to find $S t$ for the other wind speeds and wind angles of attack but are not shown for brevity. The sensitivity of the Strouhal number to the $R e$ for the bare deck pitched to different angles is illustrated in Fig. 16. 


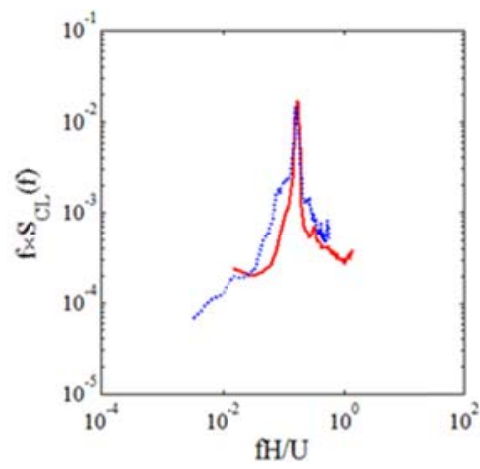

(a) Angle of attack $=-6^{\circ}$

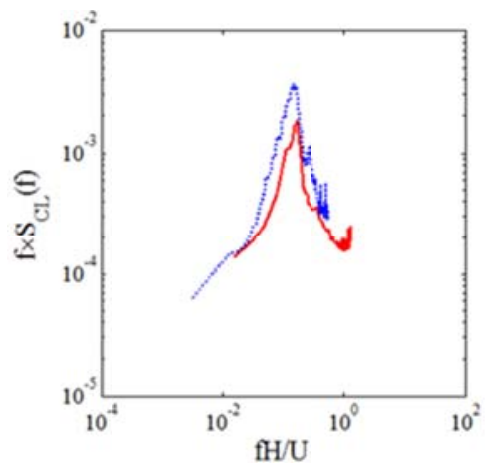

(b) Angle of attack $=0^{\circ}$

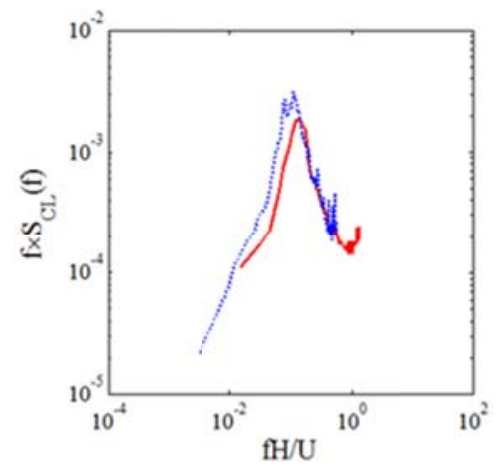

(c) Angle of attack $=+6^{\circ}$

Figure 15: Normalized lift spectra

$-1.27 \times 10^{6} \ldots \ldots \ldots . . .13 \times 10^{6}$

As shown in Fig. 15, a distinct peak is distinguished in the lift coefficient spectra showing that the bare deck cross section was subjected to vortex shedding. It is also evident that for the larger $R e$, larger peaks were observed for the zero and $+6^{\circ}$ wind angles of attack, meaning that vortices shed with higher energy at higher Re. From Fig. 16, it is noticed that $S t$ for the bare deck in the negative angles of attack was less $R e$ dependent compared to the zero and positive angles of attack. For the zero and $+3^{\circ}$ wind angle of attack, $S t$ varied inconsistently with the $R e$ increase with an overall decreasing trend. For the $+6^{\circ}$ wind angles of attack a decreasing trend was noticed.

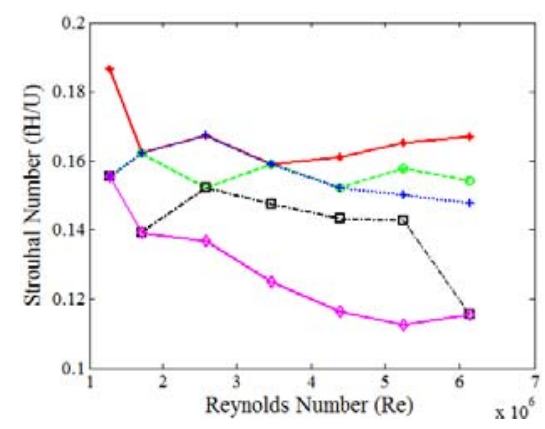

Figure 16: Strouhal number as a function of $R e$

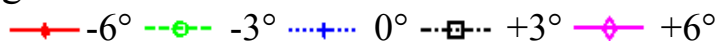


Previous literature suggests that three mechanisms may lead to vortex shedding in a twin girder bridge (Kwok et al, 2012; Laima et al, 2013). The first and second mechanisms are the flow separation over the trailing edges of the upstream and downstream girders, respectively. Third mechanism is the buffeting action of the shed vortices from the upstream girder on the downstream girder. Figure 17 shows the pressure spectra at the critical locations known as the sources of vortex shedding for the bare deck at $0^{\circ}$ angle. It is noticed that the first and third mechanisms were more critical compared to the flow separation from the trailing edge of the downstream girder. The peaks of the pressure spectra were in better agreement with $S t$ values calculated from the lift spectra at higher $R e$ (Fig. 17). This means that by increasing the $R e$, better agreement was observed between the peaks of the pressure spectra and the $S t$ values calculated from the lift spectra for all possible mechanisms of vortex shedding. 


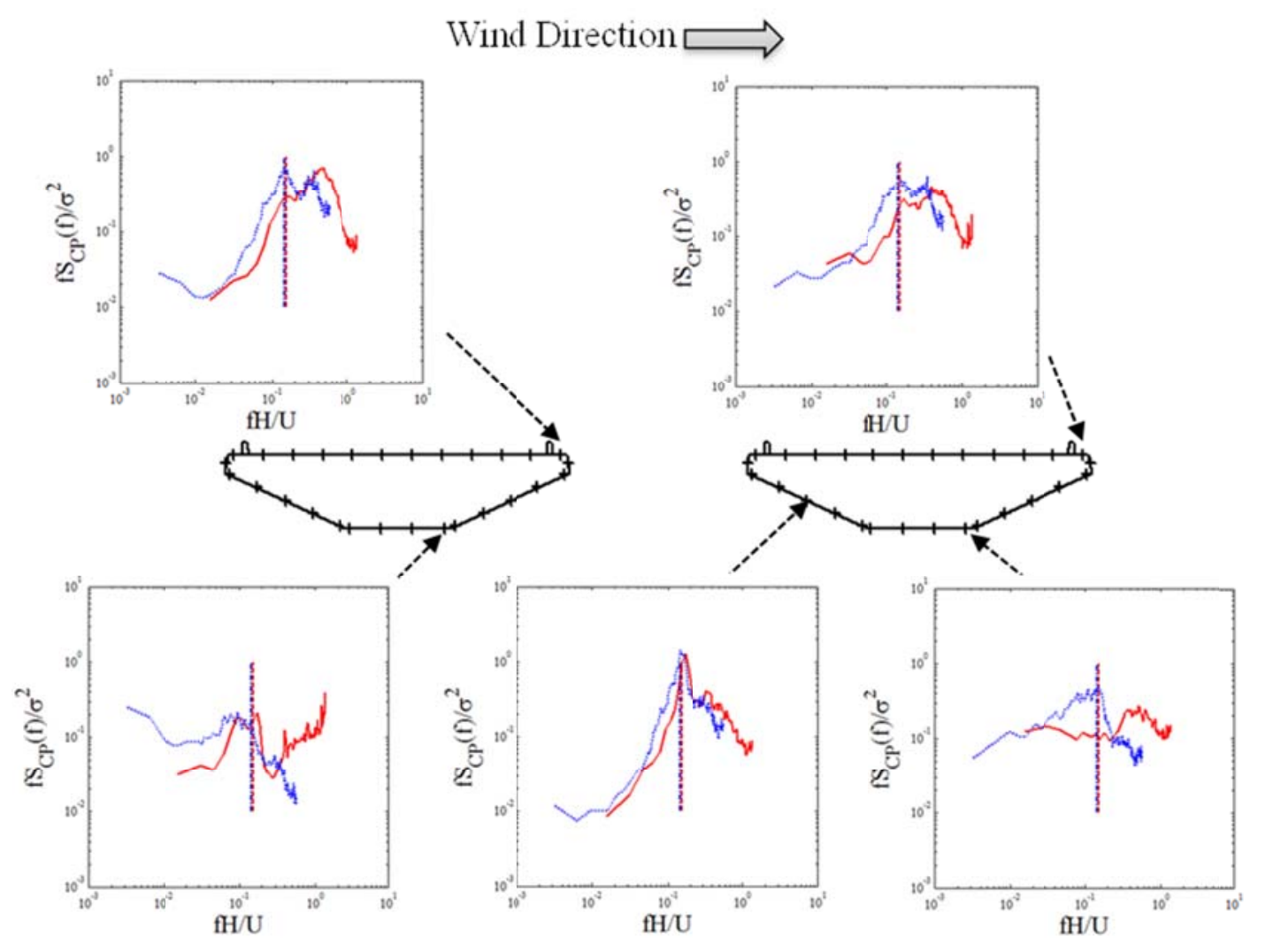

Figure 17: Pressure spectra at $0^{\circ}$ wind angle of attack (Dashed lines show the St found from lift spectra)

$-1.27 \times 10^{6} \ldots \ldots \ldots .6 .13 \times 10^{6}--1.27 \times 10^{6}--6.13 \times 10^{6}$

\subsubsection{Effect of vortex generators}

As discussed in Section 2.5.5, the bare bridge deck cross section was subjected to the vortex shedding. In order to mitigate vortex shedding, diverging pairs of rectangular plates were attached to the bottom flat surface of the two decks. Plates were placed on the upstream side of the gap to keep the approaching wind attached to the surface and guided it to flow into the gap and stopped the formation of vortices by creating a pair of counter rotating helical vortices. The sectional model fitted with the vortex generators is shown in Fig. 18. 


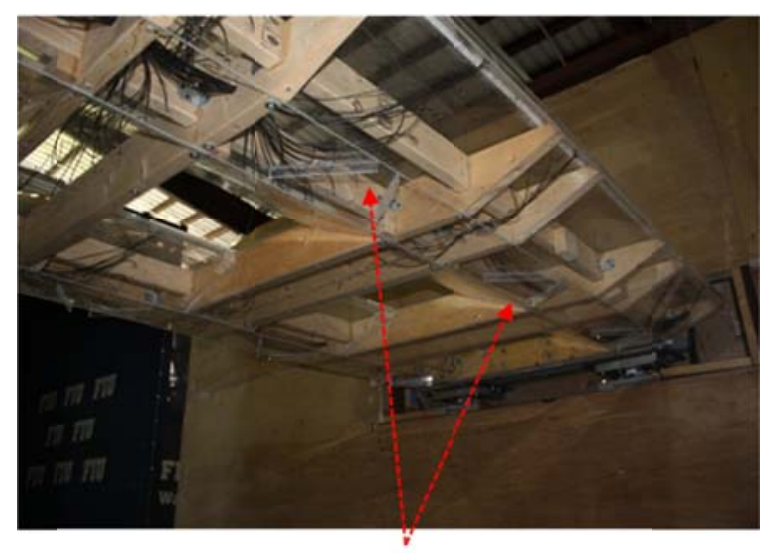

Vortex Generators

Figure 18: Vortex generators at bottom surface

Figure 19 shows the comparison of the lift coefficient power spectral density against the reduced frequency for the deck equipped with the vortex generators and the bare cross section at the maximum and minimum tested wind speeds for $-6^{\circ}, 0^{\circ}$, and $+6^{\circ}$ angles of wind attack. From Fig. 19, it is evident that adding the vortex generator to the bare section eradicated the apparent spectral peak in the lift spectrum. It seems that the mitigation devices were more effective in the low $R e$ regime, particularly for the zero and negative wind angles of attack. With the $R e$ increase a new peak formed in the lift coefficient spectrum at a smaller reduced frequency compared to the original section St. Unlike the bare section, this peak in the spectrum was distributed over a wider range of frequencies and was weaker. It is hypothesized that by increasing the $R e$, the two counter rotating vortices got mixed and flow separated from the surface. Therefore, a new wake region formed from vortices that were shed at a broad range of frequencies. 


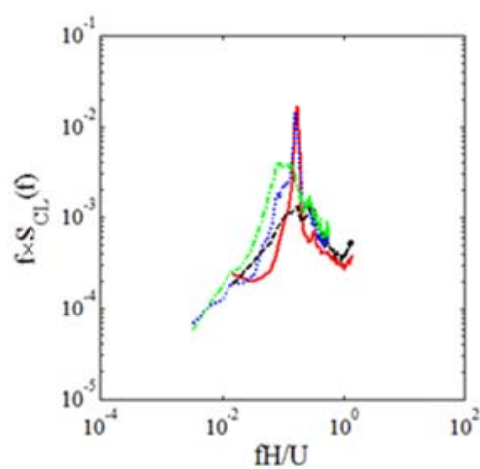

(a) Angle of attack $=-6^{\circ}$

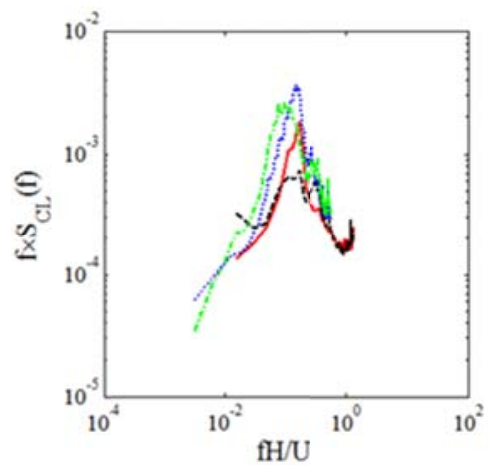

(b) Angle of attack $=0^{\circ}$

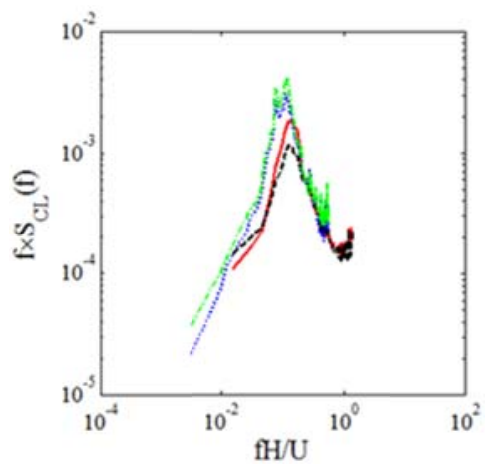

(c) Angle of attack $=+6^{\circ}$

Figure 19: Normalized lift spectra, Bare section

$-1.27 \times 10^{6} \quad \ldots \ldots . . .6 .13 \times 10^{6}$, Equipped with vortex generators ----- $1.27 \times 10^{6}$------ $6.13 \times 10^{6}$

\subsection{Conclusion}

In order to evaluate $R e$ effects on aerodynamic characteristics of a twin-deck bridge, a sectional model was tested in a uniform flow. The pressure distribution and aerodynamic forces were measured over a Reynolds number $(R e)$ range from $1.3 \times 10^{6}$ to $6.1 \times 10^{6}$ based on the deck width. The variation in the turbulence intensities was not significant and for the larger wind speeds it was almost a constant value around 3.0 percent. The following experimental observations were made:

1. The studied bridge section showed different behavior with regards to the $R e$ dependency for different angles of attack, showing higher dependency on Re for positive angles of attack.

2. Flow separated from the top surface of the upstream girder at the location of the upwind traffic barrier. For negative angles of attack the separation and reattachment points were almost fixed and the flow pattern changes due to the $R e$ were negligible.

3. At zero and $+3^{\circ}$ wind angles of attack, the structure of flow within the gap region changed by the $R e$ increase. Pressure distribution on the windward inclined surface of 
the downstream girder and on the leeward tipping edge of the upstream girder changed noticeably with $R e$.

4. For zero wind angle of attack which is the most important wind direction, the maximum drag coefficient change was noticed to be around $8 \%$ while lift coefficient changed more than $70 \%$, for the change in $R e$ values simulated.

5. The derivative of the aerodynamic force coefficients with respect to the wind angle of attack changed with $R e$ for the case of positive angles of attack. The results suggest that testing at lower $R e$ regime is conservative for aeroelastic analysis of flutter.

6. For zero and positive wind angles of attack with increases in $R e$, the distribution of the pressures around the upstream girder moved closer to the pressure distribution around the downstream girder. This means that $R e$ increase helped in sharing loads more uniformly between the two girders.

7. Vortex shedding of this twin-deck bridge was mainly governed by the vortex shedding from the trailing edge of the upstream deck and its impingement on the downstream deck. Adding a pair of diverging vortex generators to the bottom surface of the girders was effective in mitigating the vortex shedding, particularly at lower $R e$ values.

\subsection{Acknowledgements}

This research was supported by the National Science Foundation (NSF) [NSF MRI Award CMMI-0923365] and Florida Center of Excellence in Hurricane Damage Mitigation and Product Development for acquisition of instruments. The authors also would like to acknowledge Maryam Asghari Mooneghi, Walter Conklin and Roy Liu Marques of Wall of Wind, FIU for their unreserved input during testing. The findings 
reported in this article are those of the authors alone, and do not necessarily represent the views of sponsoring agencies. We also acknowledge the contribution of T.Y. Lin International in providing us with information on the East Bay Bridge. The results provided in the paper do not extrapolate directly to the East Bay Bridge as the geometry was simplified for the purposes of the present research.

\subsection{References}

Asghari Mooneghi, M., Irwin, P., Gan Chowdhury, A., 2014. Large-scale testing on wind uplift of roof pavers. Journal of Wind Engineering and Industrial Aerodynamics 128, 2236.

Barre, C., Barnaud, G., 1993. High Reynolds number simulation techniques and their application to shaped structures model test, 1st IAWE European and African Regional Conference on Wind Engineering, Guernsey UK, pp. 83-93.

Chen, X., Kareem, A., 2002. Advances in Modeling of Aerodynamic Forces on Bridge Decks. Journal of Engineering Mechanics 128, 1193-1205.

Chowdhury, A.G., Sarkar, P.P., 2004. Identification of eighteen flutter derivatives of an airfoil and a bridge deck. Wind and Structures 7, 187-202.

Gu, M., Zhang, R., Xiang, H., 2001. Parametric study on flutter derivatives of bridge decks. Engineering Structures 23, 1607-1613.

Irwin, P., Cooper, K., Girard, R., 1979. Correction of distortion effects caused by tubing systems in measurements of fluctuating pressures. Journal of Wind Engineering and Industrial Aerodynamics 5, 93-107.

K. Matsuda, K.R.C., H. Tanaka, M. Tokushige, T. Iwasaki, 2001. An investigation of Reynolds number effects on the steady and unsteady aerodynamic forces on a 1:10 scale bridge deck section model. Journal of Wind Engineering and Industrial Aerodynamics $89,619-632$.

Kazutoshi, M., Masafumi, T., Tooru, I., 2007. Reynolds Number Effects on the Steady and Unsteady Aerodynamic Forces Acting on the Bridge Deck Sections of Long-Span Suspension Bridge. IHI Engineering Review 40, 12-26.

Kubo, Y., Nogami, C., Yamaguchi, E., Kato, K., Niihara, Y., Hayashida, K., 1999. Study on Reynolds number effect of a cable-stayed bridge girder, in: A. Larsen, G.L. Larose, 
F.M. Livesey (Eds.), Wind Engineering into the 21st Century, Balkema, Rotterdam, pp. 935-940.

Kwok, K.C.S., Qin, X.R., Fok, C.H., Hitchcock, P.A., 2012. Wind-induced pressures around a sectional twin-deck bridge model: Effects of gap-width on the aerodynamic forces and vortex shedding mechanisms. Journal of Wind Engineering and Industrial Aerodynamics 110, 50-61.

Laima, S., Li, H., Chen, W., Li, F., 2013. Investigation and control of vortex-induced vibration of twin box girders. Journal of Fluids and Structures 39, 205-221.

Larose, G.L., D'Auteuil, A., 2006. On the Reynolds number sensitivity of the aerodynamics of bluff bodies with sharp edges. Journal of Wind Engineering and Industrial Aerodynamics 94, 365-376.

Larose, G.L., Larsen, S.V., Larsen, A., Hui, M., Jensen, A.G., 2003. Sectional model experience at high Reynolds number for the deck of a $1018 \mathrm{~m}$ span cable-stayed br idge, 11th International Conference on Wind Engineering, Lubbock Texas USA, pp. 373-380.

Larose, G.L., Livesey, F.M., 1997. Performance of streamlined bridge decks in relation to the aerodynamics of a flat plate. Journal of Wind Engineering and Industrial Aerodynamics 69-71, 851-860.

Larose, G.L., Wall, A., McAuliffe, B.R., Kelly, D., Stone, G., Yakymyk, W., 2012. Sectional model investigation at high Reynolds number for a super tall building. Journal of Wind Engineering and Industrial Aerodynamics 104-106, 49-55.

Larsen, A., Savage, M., Lafrenière, A., Hui, M.C.H., Larsen, S.V., 2008. Investigation of vortex response of a twin box bridge section at high and low Reynolds numbers. Journal of Wind Engineering and Industrial Aerodynamics 96, 934-944.

Lee, S., Kwon, S.-D., Yoon, J., 2014. Reynolds number sensitivity to aerodynamic forces of twin box bridge girder. Journal of Wind Engineering and Industrial Aerodynamics $127,59-68$.

Neuhaus, C., Höffer, R., 2011. Identification of quasi-stationary aeroelastic force coefficients for bridge deck sections using forced vibration wind tunnel testing, in: Roeck, G.D., Degrande, G., Lombaert, G., Muller, G. (Eds.), 8th International Conference on Structural Dynamics, EURODYN 2011, Leuven, Belgium, pp. 13861392.

Piña, R.B., Caracoglia, L., 2009. Extraction of Flutter Derivatives from Small-Scale Wind Tunnel Experiments, 11th Americas Cnference on Wind Engineering, San Juan, Puerto Rico.

Scanlan, R.H., 1978. The action of flexible bridges under wind, I: Flutter theory Journal of Sound and Vibration 60, 187-199. 
Schewe, G., 2001. Reynolds number effects in flow around a more-or-less bluff bodies. Journal of Wind Engineering an Industrial Aerodynamics 89, 1267-1289.

Schewe, G., 2009. Reynolds-Number-Effects in Flow around a rectangular Cylinder with Aspect Ratio 1:5, 5th European \& African Conferences on Wind Engineering (EACWE 5) Florence, Italy.

Schewe, G., Larsen, A., 1998. Reynolds number effects in the flow around a bluff bridge deck cross section. Journal of Wind Engineering and Industrial Aerodynamics 74-76, 829-838.

Wardlaw, R.L., Tanaka, H., Utsunomiya, H., 1983. Wind tunnel experiments on the effects of turbulence on the aerodynamic behaviour of bridge road decks. Journal of Wind Engineering and Industrial Aerodynamics 14, 247-257.

Wu, T., Kareem, A., 2012. An overview of vortex-induced vibration (VIV) of bridge decks. Frontiers of Structural and Civil Engineering 6, 335-347. 
CHAPTER III

THE EFFECTS OF TURBULENCE ON THE PRESSURE DISTRIBUTION AROUND A TWIN BOX GIRDER BRIDGE AND THE RESULTANT AERODYNAMIC FORCES

(A paper under review in The Journal of Wind Engineering and Industrial Aerodynamics) 


\section{CHAPTER III}

THE EFFECTS OF TURBULENCE ON THE PRESSURE DISTRIBUTION AROUND

A TWIN BOX GIRDER BRIDGE AND THE RESULTANT AERODYNAMIC

FORCES

Ramtin Kargarmoakhar ${ }^{\mathrm{a}},{ }^{*}$ Arindam Gan Chowdhury ${ }^{\mathrm{b}}$, Peter Irwin ${ }^{\mathrm{c}}$

\subsection{Abstract}

In order to avoid aerodynamic instability of a bridge or serviceability problems under wind loading, wind-tunnel testing is usually performed during the design to evaluate the wind effects and develop an aerodynamically stable cross section. For practical reasons it is difficult to satisfy all the similarity requirements that strictly apply in wind tunnel testing. Therefore, it is important to investigate the effects of dissimilarities between the model and the prototype due to the imperfections in the wind simulation process. Past researches on the effect of laboratory simulation of turbulence characteristics (i.e. turbulence intensity and turbulence integral length scale) on bridge aerodynamics have shown inconclusive results. This paper discusses the sensitivity of the aerodynamic behavior of a twin-deck bridge cross-section to the oncoming flow turbulence characteristics. Chord-wise pressure measurements were carried out on 1/36 and 1/72 scaled sectional models of the bridge under two flow conditions using the Wall

${ }^{a} \mathrm{PhD}$ candidate, Civil and Environmental Engineering/International Hurricane Research Center, Florida International University, Miami, FL, USA, rkarg001@,fiu.edu

b Corresponding Author, Associate Professor, Civil and Environmental Engineering/International Hurricane Research Center, Florida International University, Miami, FL, USA, chowdhur@fiu.edu, Tel: (305) 348-0518

${ }^{c}$ Professor of Practice, Civil and Environmental Engineering/International Hurricane Research Center, Florida International University, Miami, FL, USA, peairwi@fiu.edu 
of Wind (WOW) facility at Florida International University (FIU). The results from these static tests were presented in terms of pressure distributions, force coefficients, lift spectra (to study vortex shedding), and aerodynamic admittance and span-wise correlation of wind-induced loads (to study buffeting wind loadings). Based on the results, the sensitivity to turbulence characteristics of pressure distribution and force and moment coefficients were discussed. Analysis of the fluctuating lift spectra indicated that higher turbulence intensity had a mitigating effect on the vortex shedding by reducing the resulting loads. Analysis of the span-wise distribution of the wind loading showed that for a given separation length, the cross correlation of the wind loads was related to the scaling factor. This contradicts the basis of the strip assumption in estimating the buffeting loads which considers the span-wise distribution of wind-induced loads to be identical to the span-wise distribution of the approaching wind speed.

Keywords: Turbulence intensity; Turbulence integral length scale; Twin-Deck Bridge; Aerodynamic response; Vortex shedding; Buffeting

\subsection{Background and Literature Review}

Long span bridges are considered as flexible structures due to their low inherent stiffness and damping. It is therefore important to evaluate the response of long span bridges under wind induced loads for design purposes. Long span bridges can experience a range of wind excited responses from vibrations such as caused by vortex shedding and buffeting, to total instability (such as galloping and flutter). Galloping and flutter are two types of instability that are driven by self-excited or motion induced aeroelastic forces. In flutter, aeroelastic forces grow enough to modify the oscillating frequencies of the structure and cancel out the mechanical damping which can lead to failure. Vortex 
induced vibration (VIV) is an aeroelastic response which happens when the vortex shedding frequency of flow separating around the structure approaches one of the structural oscillation frequencies. VIV can result in self-limited large amplitude motions that usually occur over a narrow band of wind speeds which is lower than the design wind speed. Buffeting is a random response that concerns the fatigue and performance and is defined as the unsteady loading on a structure due to velocity fluctuations in the oncoming flow.

In order to evaluate the bridge response under wind loading, usually wind tunnel testing is performed under smooth flow condition in the initial stages of the design. The objective of the wind tunnel testing is first to come up with a cross sectional shape which is aerodynamically stable and second to predict the buffeting response. With regards to stability, it is generally believed that the results from wind tunnel testing in smooth flow condition are conservative, as the turbulence in the flow can reduce the span-wise correlation of the motion-induced forces. However, the prototype bridge is usually exposed to turbulent wind flows with turbulence intensities as great as $20 \%$ and integral scales as large as ten times their deck width. Turbulence can affect the flow separation and reattachment and therefore the surrounding flow state around the section. Based on the literature on the effects of turbulence on rectangular cylinders, it was observed that turbulence decreases the radius of curvature of separated shear layers and moves reattachment upstream (Haan Jr. et al, 1998).

One issue is that with the limitations in generating large turbulence intensity and/or scale in a wind tunnel, only a fraction of the turbulence characteristics can be simulated in the experiments. Also, there are cases in the literature that contradict each 
other regarding the turbulence effects on the bridge model response. It is therefore of importance to clarify the mechanism behind the turbulence effect on the bridge response under wind loading and to determine the minimum characteristics that need to be simulated to ensure the accuracy of the results. Most of the previous studies show that turbulence has a stabilizing effect on the aeroelastic response of a bridge deck. However, there are some cases saying that turbulence leads to a destabilizing trend on the aeroelastic response. A summary of the literature from both cases is given here. Matsumoto et al (1993) studied the effect of turbulence on VIV for a hexagonal girder and a rectangular cylinder with aspect ratio equal to 4 . They observed a different behavior between the two cases studied. They noticed a destabilizing trend by turbulence for the hexagonal model. However, turbulence was helpful in stabilizing the response for the rectangular model. They attributed the observed difference to the geometrical shape of each case study and its effect on the flow pattern.

Scanlan and Lin (1978) performed section model tests on a trussed deck section under smooth and grid generated turbulent flows. They observed that the overall response was insensitive to the turbulence and the only difference that they observed between the two cases was that the flutter derivatives were slightly larger under the turbulent wind. Gu et al (2001) also noticed that turbulence had negligible effects on the flutter derivatives for the Jiangyin Bridge which can be categorized as a streamlined single box bridge deck. Huston (1986) studied the effect of turbulent flow with large scale eddies on the aeroelastic response of the Golden Gate Bridge deck. The results showed a destabilizing trend for the turbulent flow by resulting in a negative torsional damping at lower wind speeds compared to the smooth flow. The aeroelastic behavior of the Great 
Belt East Bridge deck was evaluated using a taut strip model under smooth and turbulent flows by Larose et al (1993). Although the grid generated turbulent flow had similar turbulence intensity as the boundary layer flow, different trends were observed in the flutter derivatives variation. Results of a paper by Wardlaw et al (1983) also showed some contradictions with respect to the turbulence effects on the bridge response. For the case of the Lions Gate Bridge deck, instability was observed when the section was tested under smooth and grid generated turbulent flows. However, the same cross section only showed buffeting response when being tested under a boundary layer turbulent flow with no sign of instability. The results for the Annacis Island Bridge and a flat plate section indicated that the aeroelastic response under a grid generated turbulent flow and a boundary layer turbulent flow were comparable. Wardlaw et al (1983) also showed that the amplitudes of vortex induced vibration of the Longs Creek Bridge and Palmerston Bridge decreased with the turbulence increase in the approaching flow.

Secondary to the evaluation of the effects of turbulence on the aeroelastic stability of the bridge, it is necessary to investigate turbulence effects on buffeting wind loads. Buffeting response is created as the direct impact of the turbulence in the oncoming wind flow. Due to the complexities in the prediction of buffeting response using the time domain approaches, the spectral approach has been widely used to determine the buffeting response of bridge decks in turbulent wind flows. However, the spectral approach does depend on the available information on the bridge aerodynamic coefficients and aerodynamic admittance. Larose (2002) showed that buffeting loads were sensitive to the turbulence characteristics. It was shown that two turbulent flows 
with similar intensities but different length scales resulted in different aerodynamic admittance and span-wise coherence of wind forces.

In fact, there is a great need to evaluate the effects of turbulence features on bridges, particularly twin-deck and multiple-deck bridges which have gained popularity recently. The objective of this research was first to evaluate the effect of turbulence characteristics, i.e. turbulence intensity and turbulence integral length scale, on the mean and fluctuating pressure distribution around a twin-deck bridge deck. The pressure distribution provides insight into the local flow distribution around the section and when integrated can describe the overall aerodynamic response of the deck regarding vortex shedding and instability, through the quasi steady approach. The second objective was to study turbulence effects on the buffeting response of a twin-deck bridge and dissociate the scale effects from the turbulence intensity effects.

In order to evaluate turbulence effects on the aerodynamic behavior of modern twin-deck long span bridges, 2-D scaled models of a recently built twin-deck bridge were modeled and tested at WOW at FIU. Models at two different scales were tested in turbulent wind flows to capture the effect of integral length scale on the bridge loading. The scale models were mounted in between two streamlined end walls to ensure 2-D wind flow along the span length. Through measuring the mean and fluctuating surface pressure distributions, the steady and unsteady wind loading of the bridge were captured. Results from the tests under flows with different turbulence intensities were compared. Changes in the pressure distribution, steady/unsteady force coefficients, fluctuating lift coefficient due to vortex shedding induced and aerodynamic admittance were the main focus of this experimental study. 


\subsection{Wind Generating Facility and Wind Flow Characteristics}

The 12-fan Wall of Wind open jet facility at FIU was used to generate the wind field for the present study. WOW can generate up to a Category 5 Saffir-Simpson Scale hurricane wind speeds. It can simulate mean wind speeds and partial turbulence characteristics of real hurricane winds within a reasonable range. The nominal cross sectional dimensions of the testing section are $4.6 \mathrm{~m}$ height $\times 6.1 \mathrm{~m}$ wide. Two different flow exposures were used in this study to explore the effects of turbulence intensity. The first flow condition was the basic WOW flow without any external turbulence generating mechanism and is referred to as smooth flow in this paper (see Fig. 1(a)). In order to generate turbulence and the boundary layer wind speed distribution in height, a set of triangular spires and floor roughness elements were designed and installed just upfront the wind outlet and the resulting flow is referred to as turbulent flow in this paper (see Fig. 1(b)).

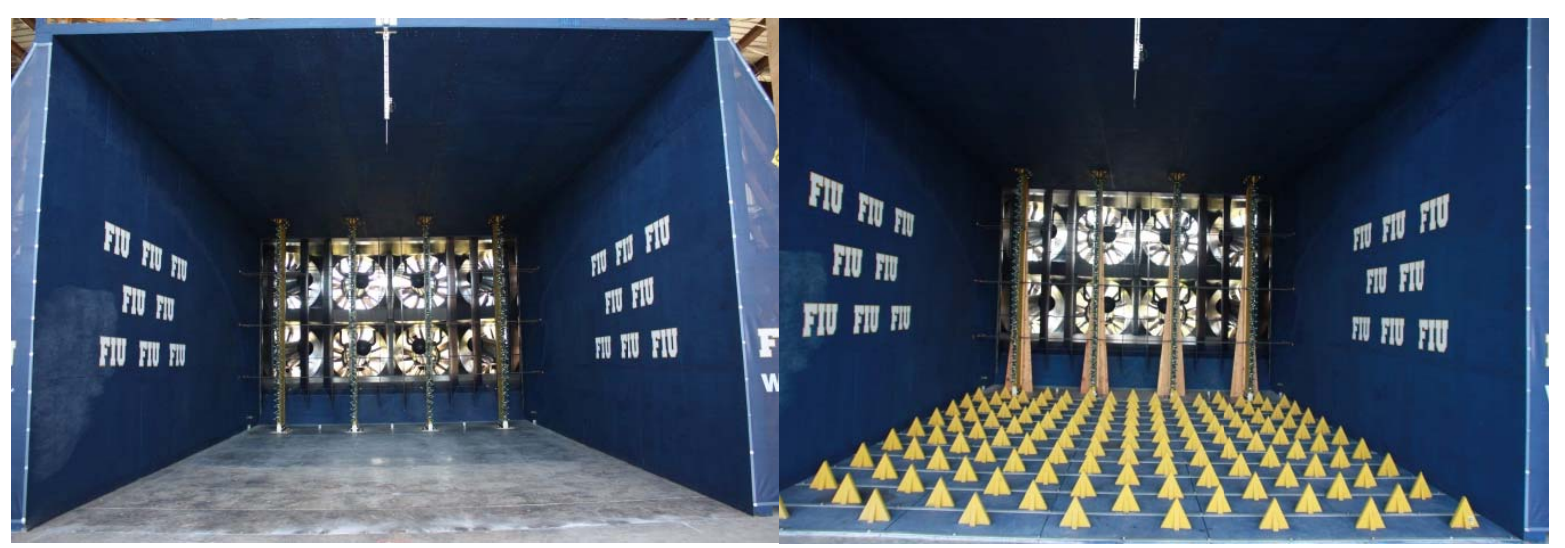

(a) Smooth flow setup

(b) Turbulent flow setup

Figure 1: Wall of Wind, Florida International University 
The bridge models were placed in between two streamlined end walls to ensure 2D air flow along the model span. The flow characteristics were measured using five three-dimensional Cobra Probes. Each Cobra Probe measured the $u, v$ and $w$ components of the wind fluctuations. Figure 2 shows the placement of the Cobra Probes during the free wind speed measurements while the bridge model was not in place. For the free wind speed measurements, a total of five Cobra Probes were used. Three Cobra Probes were installed at the bridge height and were spaced equally from each other and from the end walls (see Fig. 2). The two other Cobra Probes were placed at $0.9 \mathrm{~m}$ below and above the center Probe. These two Cobra probes also remained in place during the actual bridge model testing. The ratio between the average of the measured wind speeds at these two points during the bridge model testing and free wind speed measurements was used to correct the measured wind speed during the free wind speed measurements at bridge height for the calculations. Data measured from the Cobra probes were collected at 100 $\mathrm{Hz}$ sampling frequency for a sampling time of $120 \mathrm{~s}$ using a CompactRIO data acquisition system.

Spectral analysis was performed on the measured wind speeds. The 12000 data points measured at each probe from 2 minutes of testing at $100 \mathrm{~Hz}$ was divided into 11 blocks, each containing 1024 points. The power spectral density (PSD) was calculated for each block and the average of the 11 blocks was used as the representative wind spectrum. Each of the wind components were fitted to the Karman-type PSD function to determine the representative turbulence integral length scale. Table 1 summarizes the flow characteristics for the three components. The comparisons of the wind power spectral density between the two flow conditions (smooth versus turbulent) for the three 
components $(u, v$ and $w)$ are shown in Fig. 3. The smooth flow exposure had its maximum at frequencies almost twice the turbulent flow exposure, implying that smooth flow had a smaller length scale to be around half of the turbulent flow length scale.

Table 1: Wind flow characteristics for the two exposures

\begin{tabular}{|l|c|c|c|c|c|c|}
\hline \multirow{2}{*}{} & \multicolumn{2}{|c|}{$u$} & \multicolumn{2}{c|}{$v$} & \multicolumn{2}{c|}{$w$} \\
\cline { 2 - 7 } & $I_{u}(\%)$ & $L_{u}(\mathrm{~m})$ & $I_{v}(\%)$ & $L_{v}(\mathrm{~m})$ & $I_{w}(\%)$ & $L_{w}(\mathrm{~m})$ \\
\hline Smooth flow & 3.0 & 0.2 & 2.8 & 0.12 & 2.8 & 0.13 \\
\hline Turbulent flow & 7.0 & 0.45 & 7.2 & 0.22 & 6.3 & 0.22 \\
\hline
\end{tabular}

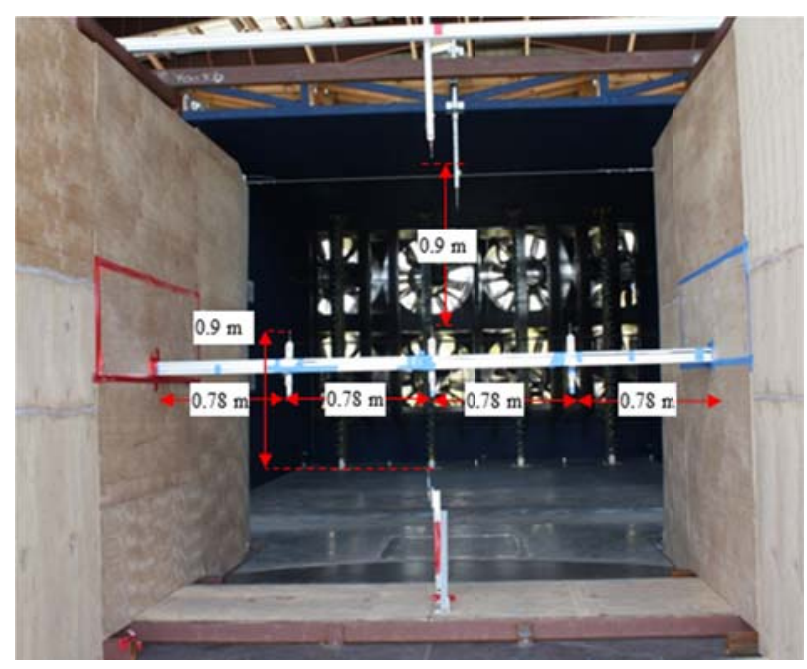

Figure 2: Placement of Cobra Probes during free wind speed measurement

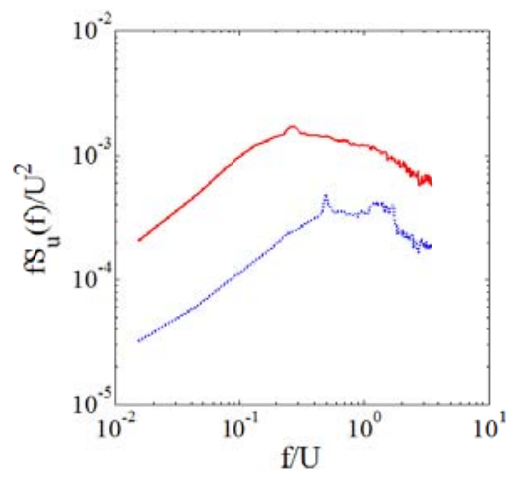

(a) $u$ component

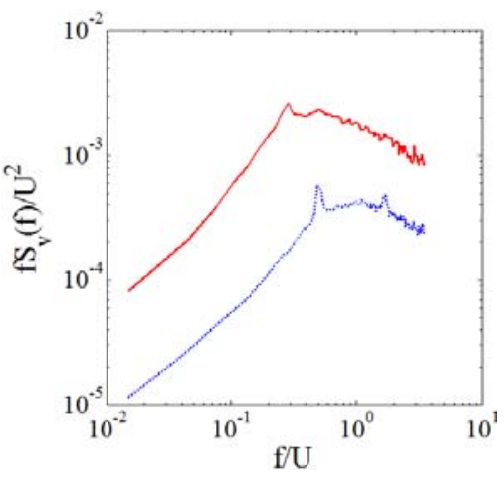

(b) $v$ component

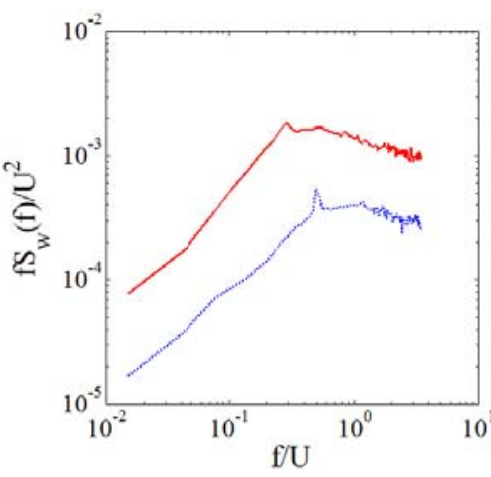

(c) $w$ component

Figure 3: Normalized PSD of the wind fluctuations

……. Smooth flow — Turbulent flow 


\subsection{Bridge Deck Section Models and Measuring Instruments}

In order to capture the effects of turbulence characteristics (turbulence intensity and turbulence integral length scale) on the pressure distribution and resulting forces for a twin-deck bridge, the simplified cross sectional shape of the eastern span of the San Francisco-Oakland bridge (East Bay Bridge) deck with the inclusion of the traffic barriers was selected. The East Bay Bridge is an asymmetric self-anchored suspension bridge with a main span of $385 \mathrm{~m}$ and a back span of $185 \mathrm{~m}$ (see Fig. 4(a)). The two decks are linked together with $10 \mathrm{~m}$ wide transverse beams every $20 \mathrm{~m}$. The single deck chord length $(b)$, total width $(B)$, gap width $(g)$ and height $(H)$ of the prototype bridge deck are $28 \mathrm{~m}, 71 \mathrm{~m}, 14 \mathrm{~m}$ and $5.5 \mathrm{~m}$, respectively (see Fig. 4(b)). The information on the bridge specifications was kindly provided by T.Y. LIN INTERNATIONAL GROUP which was involved in all phases of developing this bridge, from designing to construction.

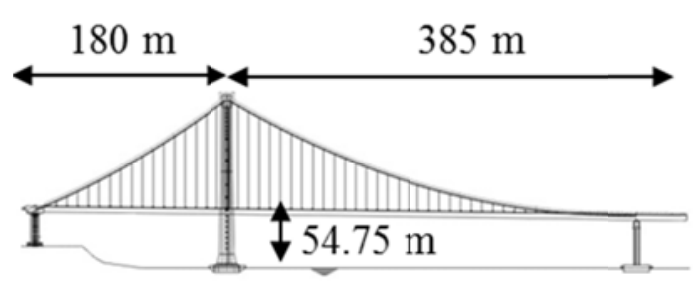

(a) General layout

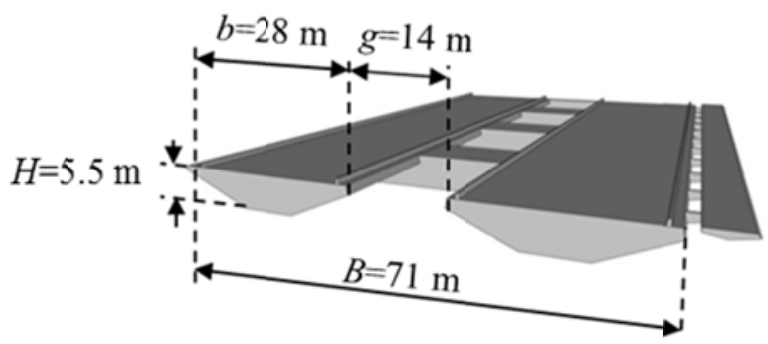

(b) Cross sectional shape

Figure 4: East Bay Bridge deck information

Sectional models at two different scales were tested to show the effect of integral length scale to single deck width ratio $\left(L_{u} / b\right)$ on the aerodynamic loading. Rigid sectional models at $1 / 36$ and $1 / 72$ scale of the prototype bridge were built and tested under the two simulated wind exposures in the WOW. Table 2 shows the ratio of the 
$\left(L_{u} / b\right)$ for the different test conditions. It can be noticed that $\left(L_{u} / b\right)$ was similar for the case of the larger model in turbulent flow and the smaller model in smooth flow. Therefore, it provided the possibility to investigate the turbulence intensity effect for a constant integral length scale to single deck width ratio.

Table 2: Ratio of the integral length scale to single deck width $\left(L_{u} / b\right)$ for different test conditions

\begin{tabular}{|c|c|c|c|c|}
\hline \multirow{2}{*}{} & \multicolumn{2}{|c|}{$1: 36$ scaled model } & \multicolumn{2}{c|}{$1: 72$ scaled model } \\
\cline { 2 - 5 } & Smooth flow & Turbulent flow & Smooth flow & Turbulent flow \\
\hline$\left(L_{u} / b\right)$ & 0.26 & 0.59 & 0.53 & 1.18 \\
\hline
\end{tabular}

Both sectional models had a similar span length which was equal to $3.05 \mathrm{~m}$ and were rigidly mounted in between the two end walls (see Fig. 5). Each model was constructed out of a wooden frame which was covered by Plexiglas plates. The models were fixed to supports on each side using a system composed of aluminum plates, pipes and I-beams. The connection system was devised so that the model section could be studied under different wind angles of attack. For the sake of this study, section models were tested at five different wind angles of attack, including $-6^{\circ},-3^{\circ}, 0^{\circ},+3^{\circ}$, and $+6^{\circ}$. Figure 6 shows how the positive and negative wind angles of attack are defined in this paper. 


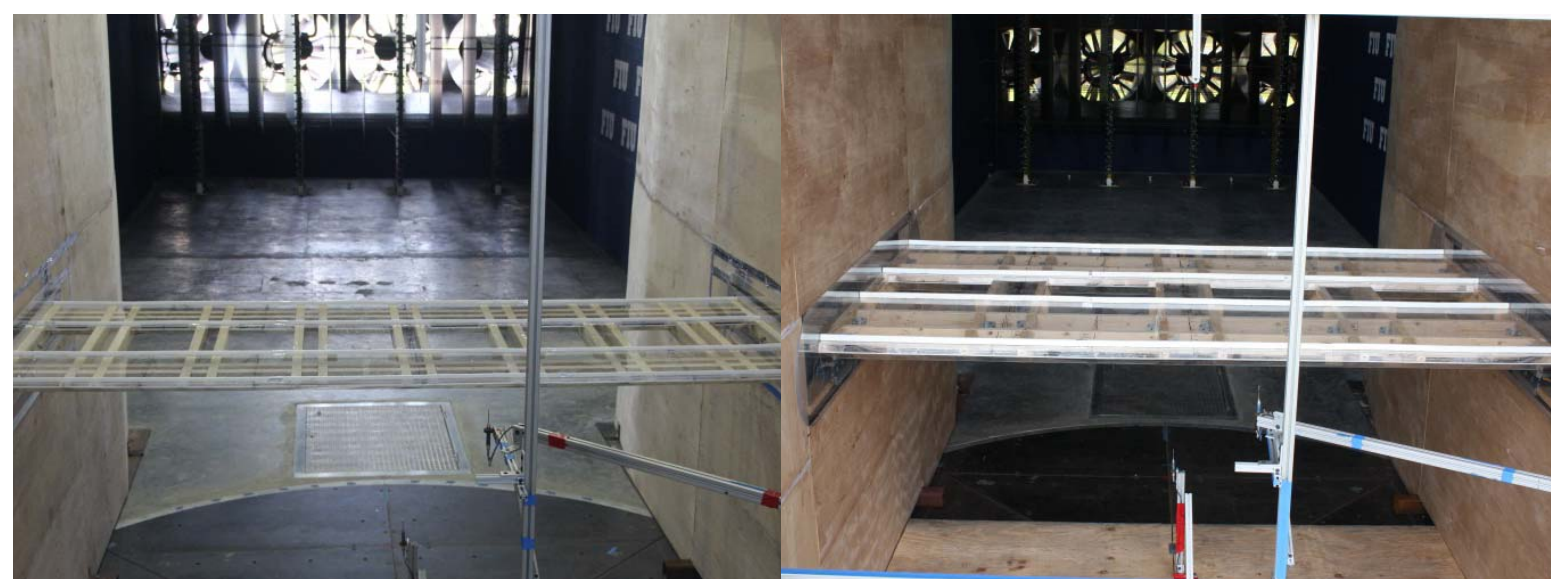

$1 / 72$ scaled model

$1 / 36$ scaled model

Figure 5: Test setup under smooth flow condition

Surface pressures were measured around chord-wise strips of each model by fitting pressure taps around each model as shown in Fig. 6. A Scanivalve Corporation pressure scanning system was used to measure pressures at a sampling frequency of 512 $\mathrm{Hz}$ for a period of two minutes for each test case. In order to reduce the pressure tube length, pressure scanners were placed at the end of the model on each side and each tap was connected to the pressure scanning system in the shortest distance. A transfer function designed for the tubing (Irwin et al, 1979) was used to correct for the tubing effects. Pressures were measured at a total of 104 points distributed around four strips and at a total of 280 points distributed around six strips for the $1 / 72$ and 1/36 scaled models, respectively. Figure 7 shows the distribution of the pressure tapped strips along the span for each model. 


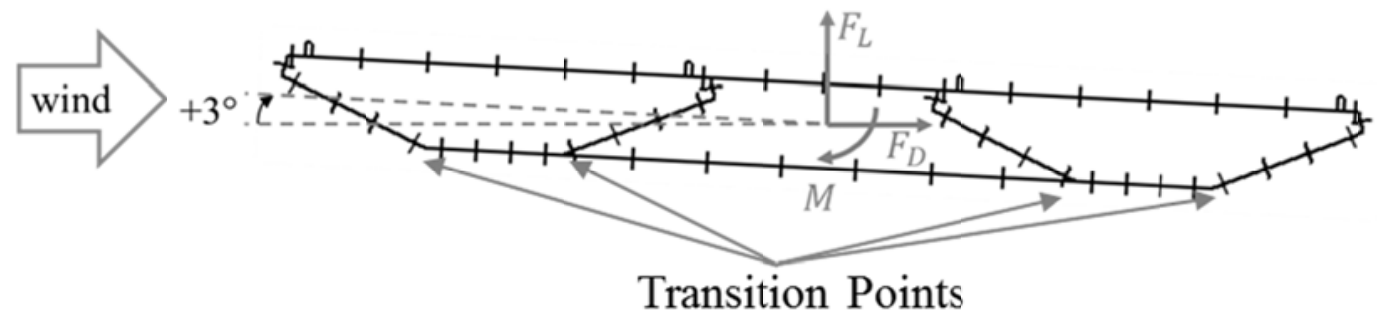

(a) $1 / 72$ scaled model

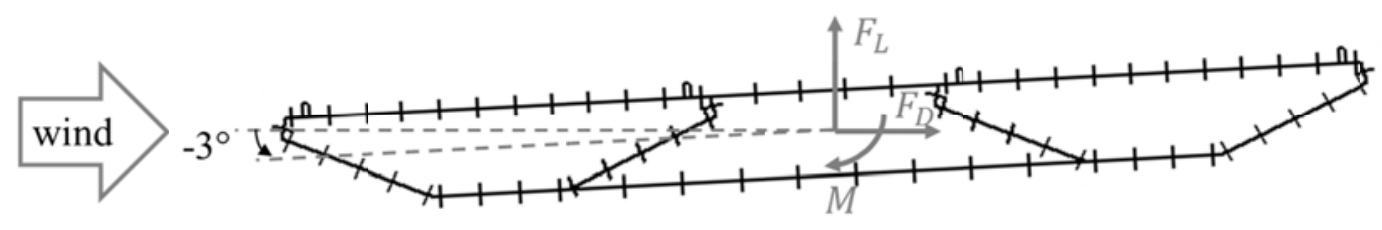

(b) $1 / 36$ scaled model

Figure 6: Chord-wise distribution of the pressure taps around the section model

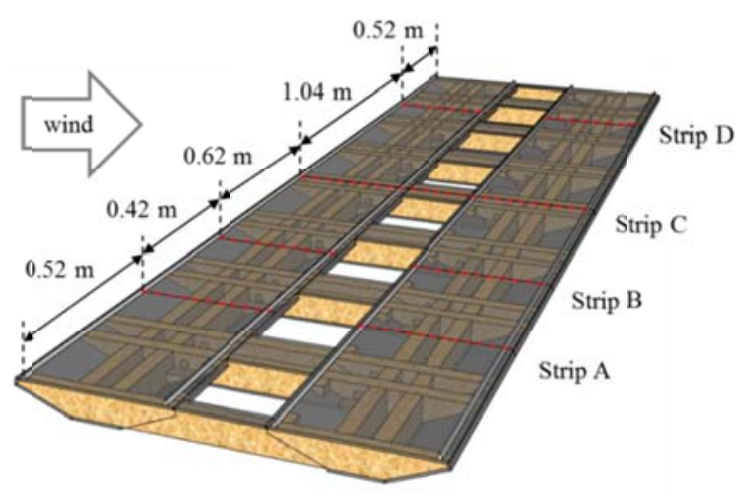

(a) $1 / 72$ scaled model

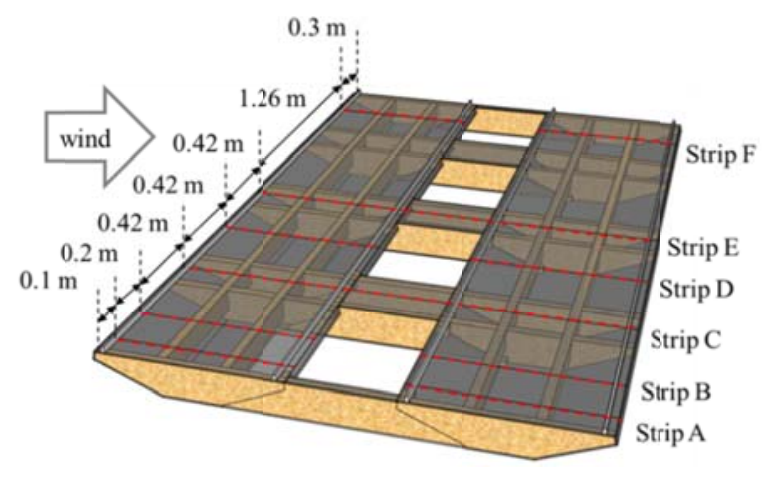

(b) $1 / 36$ scaled model

Figure 7: Distribution of the pressure tapped chord-wise strips along the span length

\subsection{Effects of Turbulence Characteristics on Pressure Distribution}

In this study, the pressure distribution around the section is presented to highlight the effect of turbulence in the oncoming flow on the local pressure changes. The pressure distribution can give an insight on the flow separation and reattachment zones (Kwok et 
al, 2012; Li and Melbourne, 1995), e.g. large negative mean pressures correspond to a separation region and maximum fluctuating pressures occur near reattachment zones. Figures 8 and 9 show the effect of turbulence intensity and turbulence integral length scale on the mean pressure coefficient distribution, respectively. The mean pressures are plotted in non-dimensional form by dividing the mean pressure collected at each pressure tap by the mean dynamic pressure $\left(1 / 2 \rho U^{2}\right.$, where $\rho$ and $U$ are the air density and mean wind speed, respectively). All the results presented in this section were based on the measured pressures at Reynolds number $(R e)$ close to $1.7 \times 10^{6}$, based on the total width.

From Fig. 8, it can be noticed that higher turbulence intensities bring the peak of the pressure distribution on the top surface of the upstream deck towards the leading edge. This was accompanied by shortening of the reattachment length for the higher turbulence intensities. For the zero and negative wind angles of attack, pressures on the bottom surface of the upstream deck and across the downstream deck were not sensitive to the turbulence intensity. For the $+6^{\circ}$ wind angle of attack, larger negative pressures were noticed near the transition points on the bottom surfaces in the smooth flow. The differences observed between the two flow conditions near the transition points suggest that wind induced excitation mechanisms may alter with the turbulence intensity.

Figure 9 shows the sensitivity to turbulence integral length scale to width ratio $\left(L_{u} / b\right)$ for the two flow conditions. In both flow conditions, larger negative pressures were formed near the transition points for the smaller $L_{u} / b$. This suggests that turbulent flows with similar turbulent intensities and smaller integral length scales resulted in the formation of larger negative pressures on the bottom surfaces of a twin deck section, 
corresponding to more highly curved streamlines at the edges of separation bubbles. Based on the results presented here, the flow condition around the section was affected by both the turbulence intensity and turbulence integral length scale.
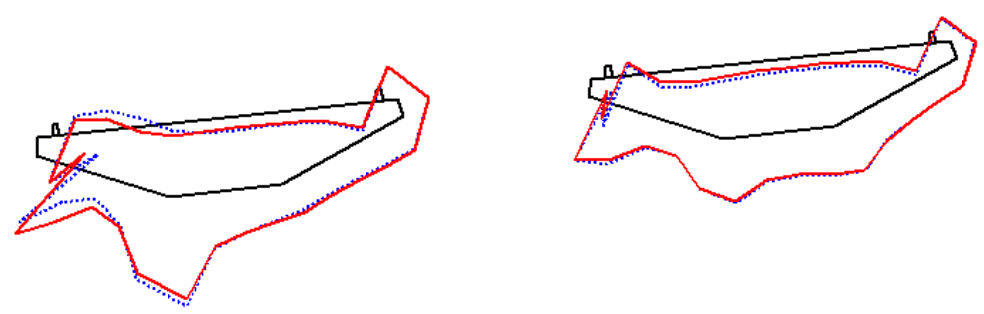

(a) Angle of attack $=-6^{\circ}$
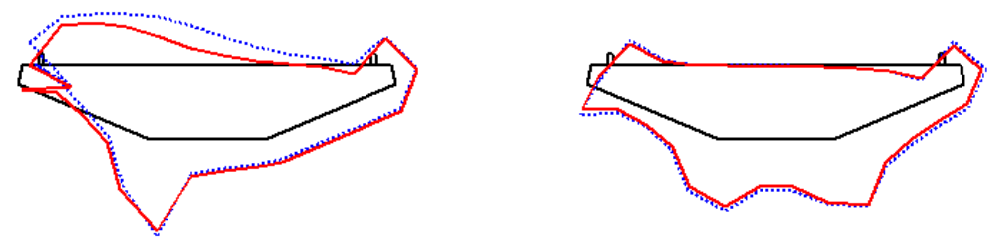

(b) Angle of attack $=0^{\circ}$
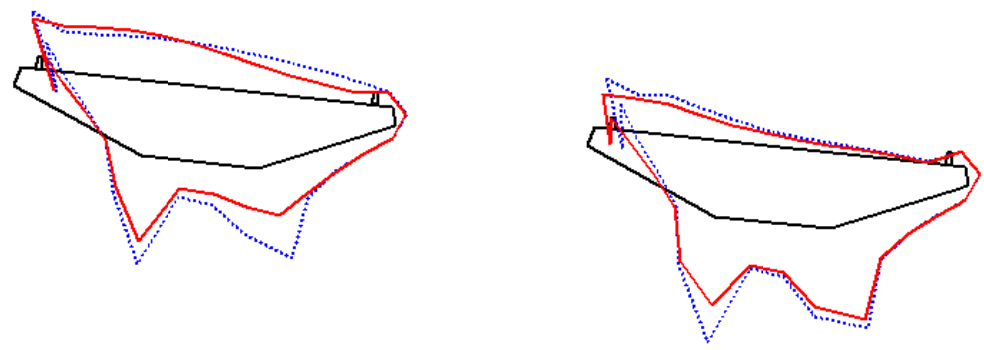

(c) Angle of attack $=+6^{\circ}$

Figure 8: Mean pressure coefficient distribution, $1 / 36$ scale model, $R e=1.7 \times 10^{6}$ $\left(C_{P}=1.0, \bullet-\cdots\right), \cdots \cdots \cdot$.... Smooth flow $\longrightarrow$ Turbulent flow 

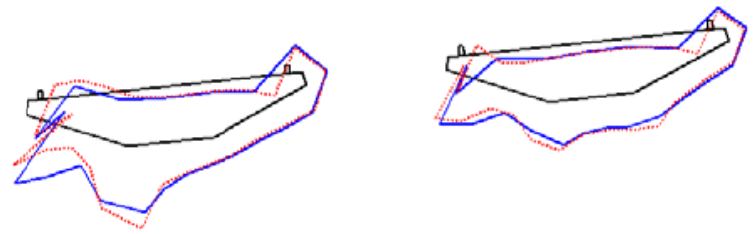

(a) Angle of attack $=-6^{\circ}$, Smooth flow
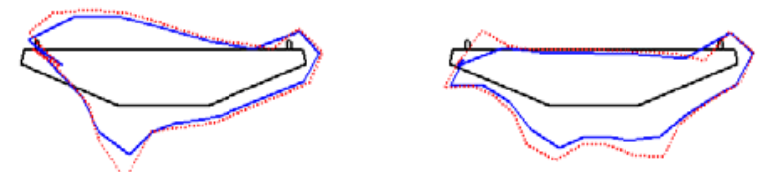

(b) Angle of attack $=0^{\circ}$, Smooth flow
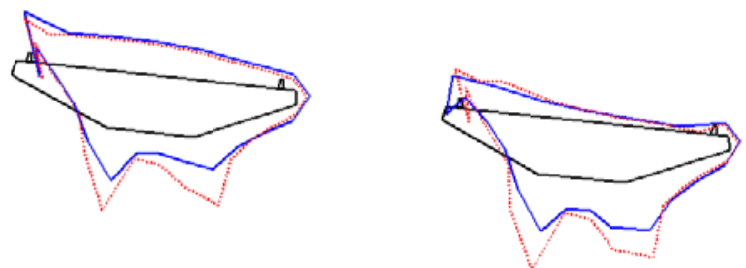

(c) Angle of attack $=+6^{\circ}$, Smooth flow
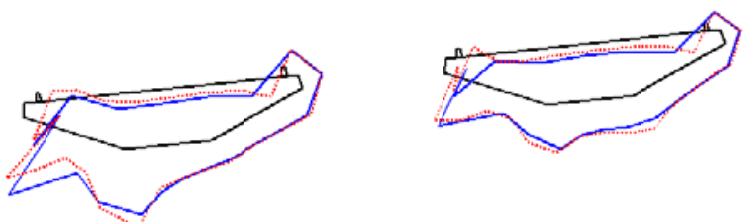
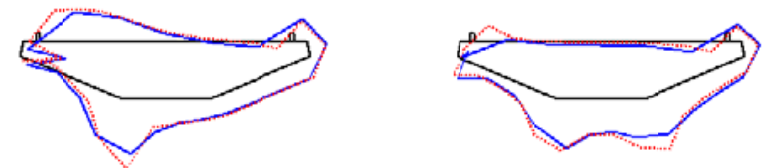

(e) Angle of attack $=0^{\circ}$, Turbulent flow
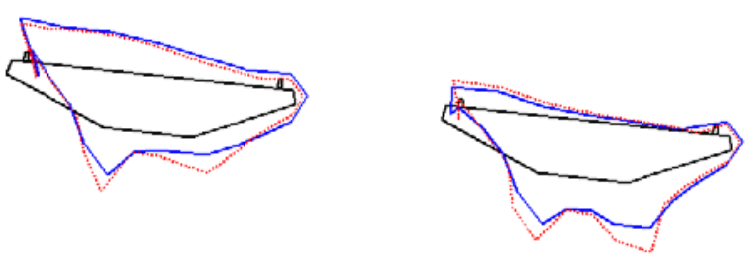

(f) Angle of attack $=+6^{\circ}$, Turbulent flow

Figure 9: Mean pressure coefficient distribution, $R e=1.7 \times 10^{6}$

$\left(C_{P}=1.0, \ldots\right),-1 / 72$ scale model $\cdots \cdots \cdot \cdots \cdot \cdots \cdot 1 / 36$ scale model

\subsection{Effects of Turbulence Characteristics on Force Coefficients}

Wind-induced static loads on long span bridges need to be evaluated for the structural design and to evaluate the potential for torsional divergence. Larger drag will lead to the design of larger and stiffer members for bridge deck elements, cables and the foundation. It has also been shown that static loads and their non-dimensionalized form can be used to describe some of the dynamic features of the wind effects on bridges using quasi steady assumptions (Larose, 2002). It is therefore of interest to evaluate the effect of turbulence characteristics on the mean and fluctuating forces and moment coefficients, 
as they can provide an insight on how these characteristics can change the global behavior of the bridge under wind.

Wind generated loads was measured at a number of wind speeds to capture the simultaneous effects of turbulence characteristics and $R e$. Measured pressures across the chord-wise length were integrated over their effective length to capture the mean and fluctuating forces and moments at each strip location. In order to reduce the randomness error, results were averaged over the different strips to obtain the effective forces and moment per unit length. Figure 10 shows the variation of the mean drag, lift and moment coefficients with $R e$ for the different test setups. Forces were expressed in a coordinate system fixed to the flow direction, i.e. drag and lift were defined in the along wind and perpendicular to wind directions, respectively (see Fig. 6). In order to obtain the mean force coefficients, the spatial averaged forces over the different strips were divided by the mean dynamic pressure and the model dimension perpendicular to the force component direction, which is the model height for the drag force and the model width for the lift force. The moment coefficient was also calculated by dividing the measured moment about the deck geometric center by the dynamic pressure and the model width squared.

From Fig. 10, it can be noticed that the mean forces and moment coefficients were sensitive to both the turbulence characteristics and $R e$, with the highest sensitivity attributed to the turbulence intensity. With turbulence intensity increase, drag and moment coefficients decreased while the lift coefficient showed an increasing trend in magnitude. A larger integral length scale to width ratio resulted in larger drag and lift coefficients in the turbulent flow. However, larger integral length scale to width ratio resulted in smaller drag and lift coefficients in the smooth flow. Different trends were 
noticed in the variation of the lift and drag coefficients with the $R e$ increase. While higher $R e$ was accompanied by larger negative lifts in almost all the testing setups, such a consistent trend was not observed in the case of drag and moment coefficients.

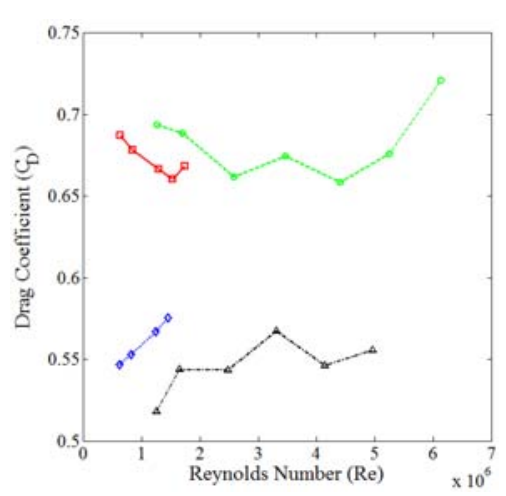

(a) Drag coefficient

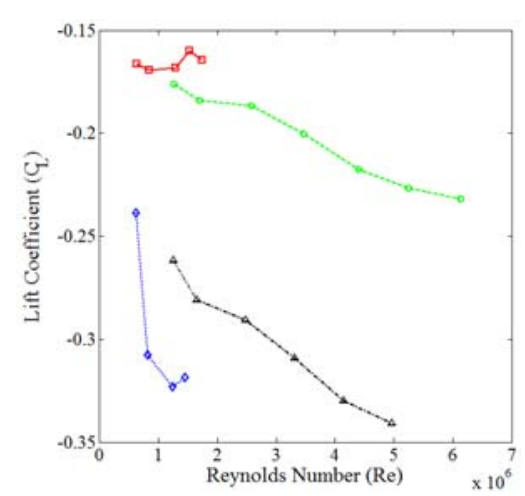

(b) Lift coefficient

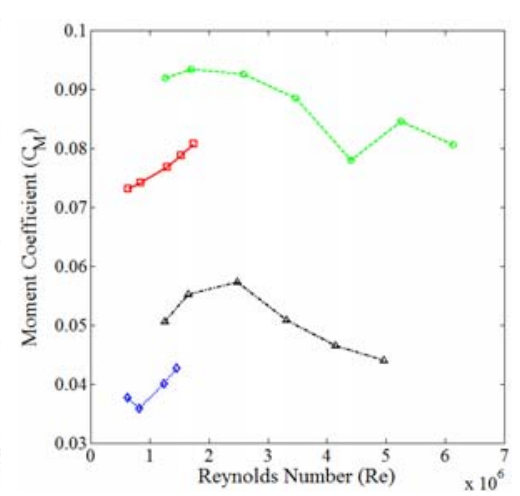

(c) Moment coefficient

Figure 10: Force and moment coefficients as a function of Reynolds number at $0^{\circ}$ angle of attack

ד- Smooth flow-1/72 scale $\left(L_{u} / b=0.53\right)$-- - - . Smooth flow-1/36 scale $\left(L_{u} / b=0.26\right)$

$\cdots \cdots \cdots$ Turbulent flow-1/72 scale $\left(L_{u} / b=1.18\right) \cdots \cdots$. Turbulent flow-1/36 scale $\left(L_{u} / b=0.59\right)$

In order to investigate the bridge response in a turbulent flow using a wind simulating facility that cannot reproduce the full turbulence length scale requirements, Partial Turbulence Simulation (PTS) was introduced by Irwin (1998). This method is based on the assumption that the flow structure around a bluff structure is strongly influenced by the small scale eddies and therefore the bridge response under a turbulent wind can be predicted relatively accurately by simulating the high frequency component of the turbulent flow at the correct energy density relative to the mean flow (Irwin, 2004). In cases where the real turbulence features, i.e. turbulence intensity and turbulence integral length scale to bridge width ratio, cannot be simulated using a wind generating facility, the bridge deck response can be predicted using PTS approach. The PTS 
approach that requires the similitude of the high frequency part of the wind spectrum can be expressed by the following equation:

$$
\frac{\left(I_{u}\right)_{m}}{\left(I_{u}\right)_{p}}=\left(\frac{\left(L_{u} x / D\right)_{m}}{\left(L_{u}{ }^{x} / D\right)_{p}}\right)^{1 / 3}
$$

where $m$ stands for model and $p$ stands for prototype.

Katsuchi and Yamada (2011) proposed a non-dimensional parameter, called reduced turbulence intensity $\left(I_{r}\right)$, based on the PTS approach. The reduced turbulence intensity can be obtained from the equation below:

$$
I_{r}=\frac{I_{u}}{\left(L_{u}^{x} / D\right)^{\frac{1}{3}}}
$$

From eq. (1), it can be seen that predicting the bridge response using the PTS approach requires that the reduced turbulence intensity to be equal between the model and the prototype. Katsuchi and Yamada (2011) observed that some of the aerodynamic features of a bridge deck, such as the maximum mean pressure and force coefficient derivatives, show a linear trend with the reduced turbulence intensity variation. In order to satisfy the PTS approach requirement which is the similitude of the reduced turbulence intensity, either the scaling factor or flow parameters should be chosen such that eq. (1) is satisfied. However, due to the limitations in the wind tunnel studies regarding the turbulence features and the size of the model (larger model is preferred to increase the simulated $R e$ ), it might be possible that eq. (1) cannot get satisfied. It is hypothesized that in cases that the reduced turbulence intensity similitude cannot be satisfied, tests can be performed at two reduced turbulence conditions available and from a linear regression, the intended parameter can be estimated based on the prototype's reduced turbulence intensity. Further investigation is however necessary to evaluate the validity of this 
hypothetical method to obtain the aerodynamic response of a bridge under a turbulent wind flow.

Figure 11 shows the variation of the force coefficients with respect to the reduced turbulence intensity at two different $R e$. It can be seen that the force coefficients were sensitive to the reduced turbulence intensity and showed almost a linear relationship with $I_{r}$. The mean drag coefficient showed a decreasing trend while the negative mean lift coefficient increased in magnitude with the $I_{r}$ increase. The force coefficient variation under different turbulent flow conditions was measured to be as large as $34 \%$ for the drag and more than $100 \%$ for the lift coefficient.

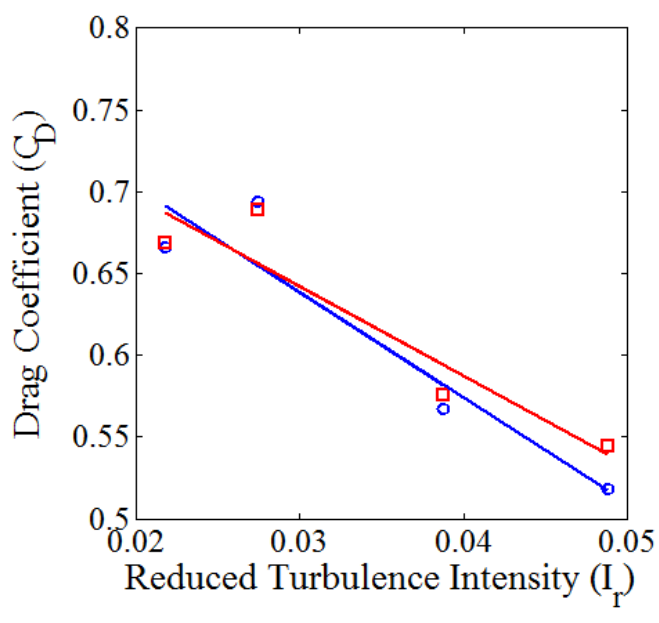

(a) Drag coefficient

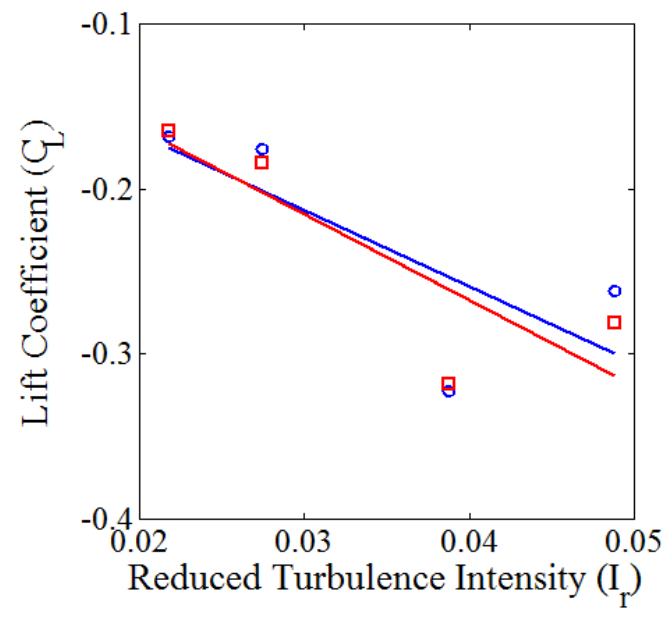

(b) Lift coefficient

Figure 11: Force coefficients as a function of reduced turbulence intensity for $0^{\circ}$ angle of attack, $\circ R e=1.3 \times 10^{6}$ ㅁ $R e=1.7 \times 10^{6}$

Figure 12 shows the derivatives of the force and moment coefficients with respect to the angle of attack, as a function of the reduced turbulence intensity for the zero wind angle of attack. While $d C_{D} / d \alpha$ showed a decreasing trend with the $I_{r}$ increase, $d C_{L} / d \alpha$ and $d C_{M} / d \alpha$ were less sensitive to the $I_{r}$ variation. It can be seen that the trend of 
variations was almost similar for the two different $R e$ regimes. The importance of the $d C_{D} / d \alpha, d C_{L} / d \alpha$ and $d C_{M} / d \alpha$ on the aerodynamic response of the bridge can be illustrated from the quasi-steady theory (Irwin, 1977). Based on the quasi-steady theory, the derivatives of the force and moment coefficients, when combined with the structural properties in the form of the natural modes of vibration, can be used to define the buffeting and wind motion-induced loads on the structure. It is understood that the quasisteady theory does not incorporate the unsteady aerodynamic coefficients that are necessary to define the aerodynamic damping and stiffness of the system. However, it is a good tool to investigate the aerodynamic features of the structure using the steady force coefficients only, particularly for the vertical and horizontal degrees of freedom. In all cases $\left(d C_{D} / d \alpha, d C_{L} / d \alpha\right.$ and $\left.d C_{M} / d \alpha\right)$, larger magnitude is associated with a larger buffeting load due to the across wind component of turbulence. Negative $d C_{L} / d \alpha$ can lead to a negative aerodynamic damping and can result in galloping instability if the total damping goes negative at a certain wind speed.

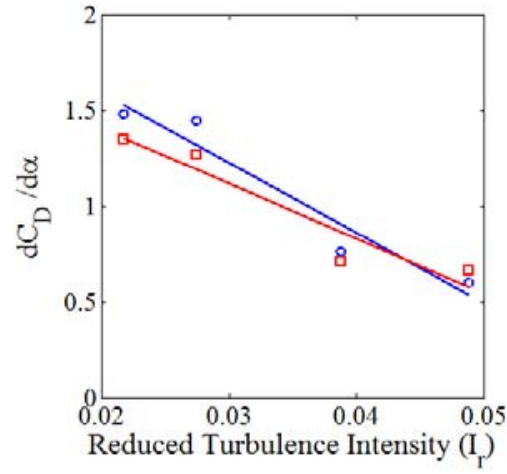

(a) $d C_{D} / d \alpha$

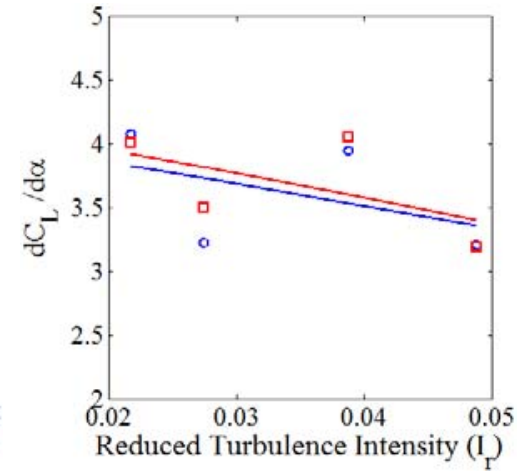

(b) $d C_{L} / d \alpha$

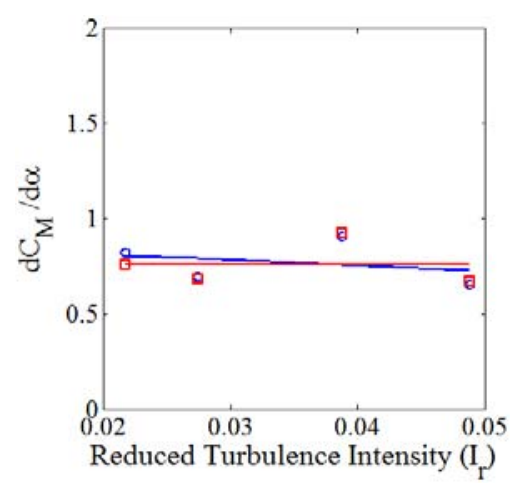

(c) $d C_{M} / d \alpha$

Figure 12: Forces and moment coefficients derivatives with respect to the angle of attack for the zero wind angle of attack

$$
\multimap R e=1.3 \times 10^{6} \multimap-R e=1.7 \times 10^{6}
$$




\subsection{Effects of Turbulence Characteristics on Vortex Shedding}

When wind blows over bluff bodies, usually flow separates at sharp edges, leading to the formation of alternating vortices which are periodically shed into the wake of the structure. Due to the shedding of vortices, periodic forces can be generated that can result in vibrations perpendicular to the direction of wind flow. When the frequency of vortex shedding gets close to the natural frequency of one of the structural vibration modes, significant vibrations may occur. These lateral vibrations have a strong organizing effect on the vortex shedding pattern which can increase the strength of the vortices and couple the vortex shedding frequency to the natural frequency of the structure. This phenomenon is known as lock-in. The amplitude of the structural response under vortex-induced vibration depends on the structural damping level associated with the structure's dominant modes of vibration. It has been shown that eventually the interaction of the structure's motion with the vortex shedding tends to limit the maximum amplitude of vortex induced vibrations (Blevins, 2001). Despite the fact that VIV is a kind of limited amplitude vibration, it can be important on bridges as it can affect the driving comfort in the short term and cause fatigue damage in long term. A number of bridges have been susceptible to VIV (Frandsen, 2001; Larsen et al, 2000).

Spectral analysis was performed on the fluctuating lifts on the models in different test setups to evaluate the effect of turbulence characteristics on the vortex shedding. Figure 13 shows the lift coefficient power spectral density against the reduced frequency for the $1 / 72$ scaled model in the smooth flow, at $-6^{\circ}, 0^{\circ}$ and $+6^{\circ}$ angles of attack. It can be seen that the model experienced a significant vortex shedding when tilted to $-6^{\circ}$ wind 
angle of attack. The lift coefficient power spectral density for the different test setups at $-6^{\circ}$ wind angle of attack is shown in Fig. 14. The peak evident in the fluctuating lift spectrum shows that the cross section was susceptible to vortex shedding in all cases. The area under the peak was measured to determine the fluctuating lift coefficient due to the vortex shedding for each test condition. Table 3 summarizes the standard deviation of the fluctuating lift coefficients due to the vortex shedding for the different test conditions at $-6^{\circ}$ wind angle of attack. Based on the results, the vortex shedding induced fluctuating lift coefficient was sensitive to both the turbulence intensity and turbulence integral length scale. It appears that increasing the turbulence intensity reduced the generated fluctuating lift coefficient while a larger fluctuating lift coefficient was noticed for larger integral length scale to bridge width ratios.

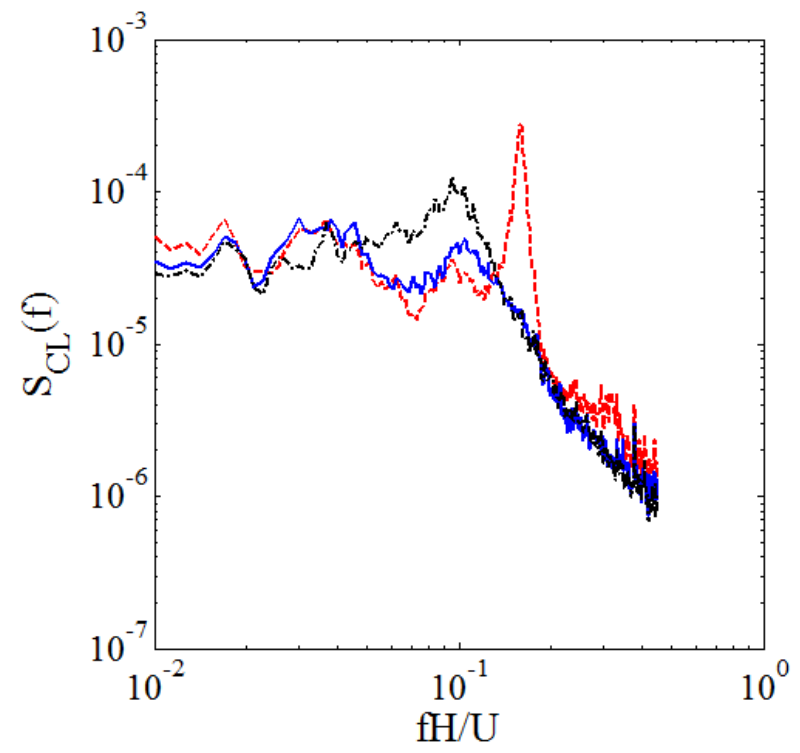

Figure 13: Lift coefficient power spectral density at $R e=1.7 \times 10^{6}$, ----- Angle of attack $=-6^{\circ}-$ Angle of attack $=0^{\circ}-\cdots \cdot-\cdot$ Angle of attack $=+6^{\circ}$ 


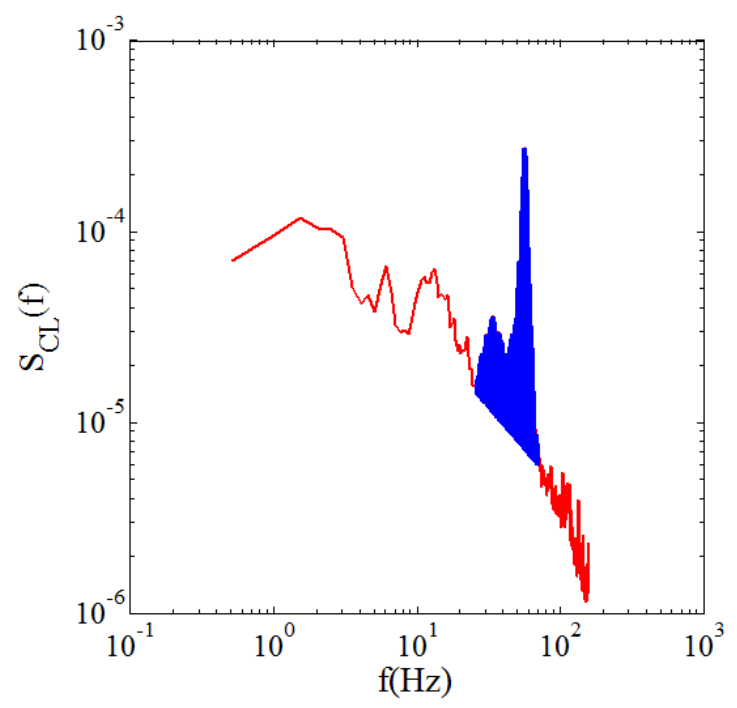

(a) Smooth flow-1/72 scale $\left(L_{u} / b=0.53\right)$

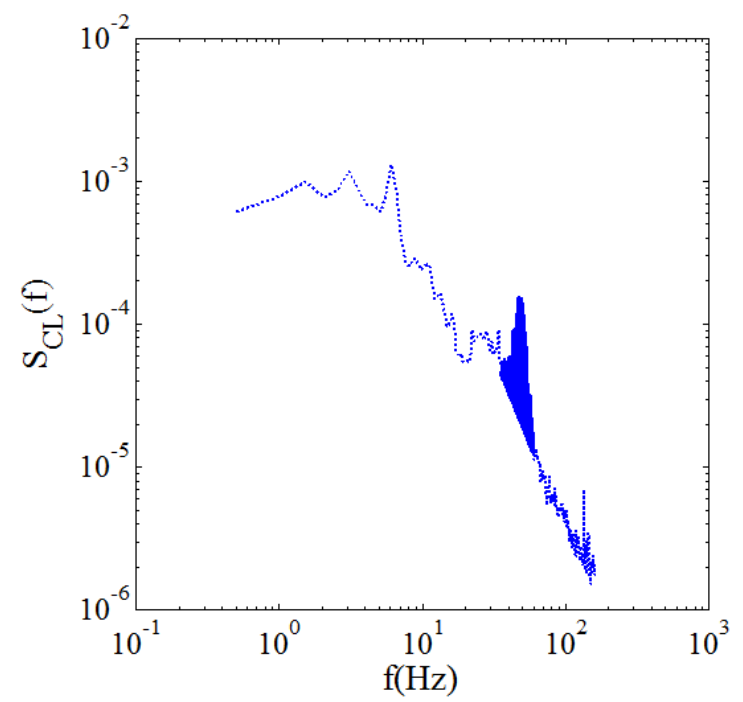

(c) Turbulent flow-1/72 scale $\left(L_{u} / b=1.18\right)$

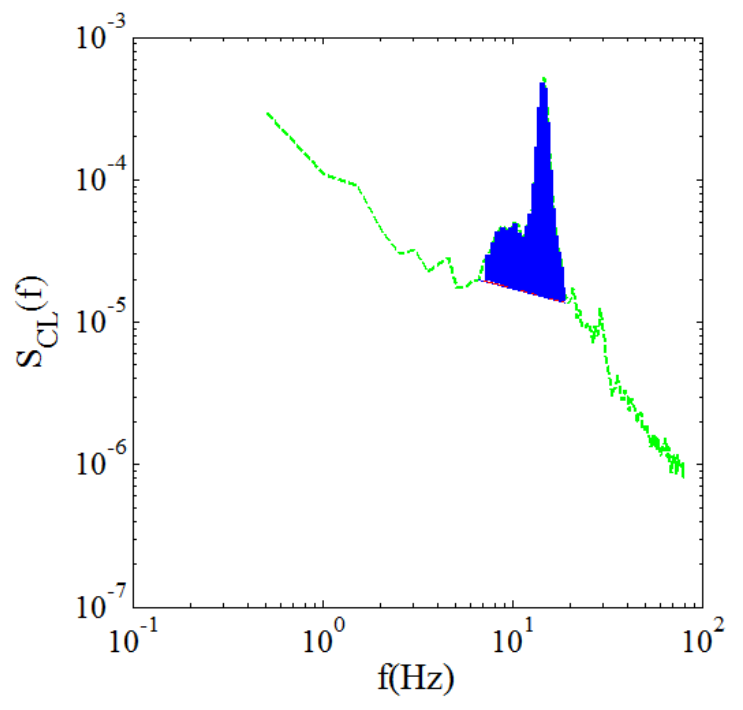

(b) Smooth flow-1/36 scale $\left(L_{u} / b=0.26\right)$

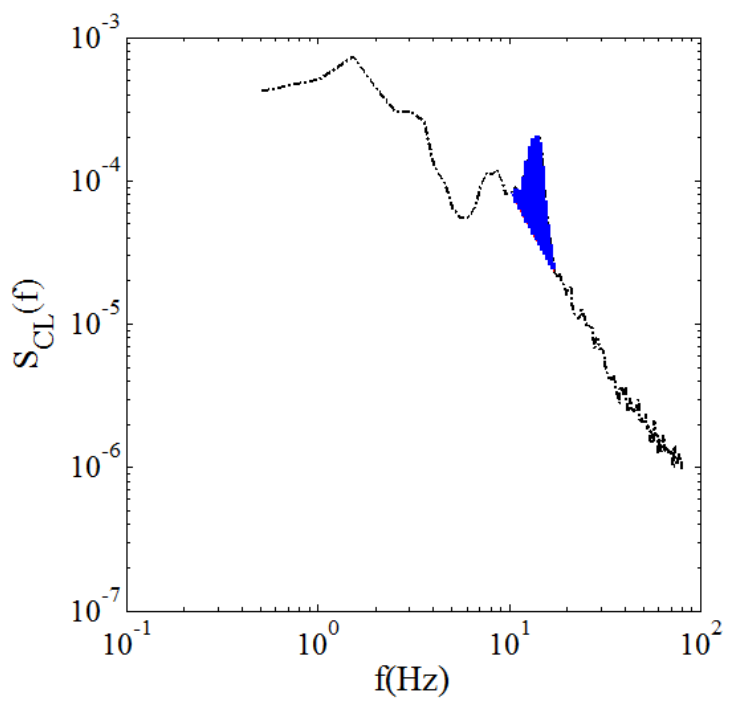

(d) Turbulent flow-1/36 scale $\left(L_{u} / b=0.59\right)$

Figure 14: Lift coefficient power spectral density at $-6^{\circ}$ wind angle of attack

$$
\left(R e=1.7 \times 10^{6}\right)
$$

Table 3: Standard deviation of the fluctuating lift coefficient due to the vortex shedding

\begin{tabular}{|c|c|c|c|c|}
\hline & $L_{u} / b=0.26$ & $L_{u} / b=0.53$ & $L_{u} / b=0.59$ & $L_{u} / b=1.18$ \\
& $I=3 \%$ & $I=3 \%$ & $I=6 \%$ & $I=6 \%$ \\
\hline$C_{L}{ }^{\prime}$ & 0.033 & 0.042 & 0.022 & 0.033 \\
\hline
\end{tabular}




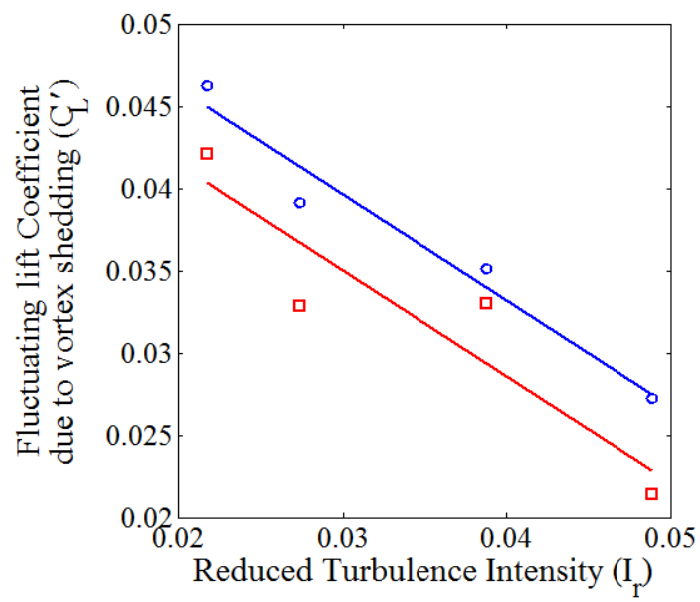

Figure 15: Standard deviation of the fluctuating lift coefficient due to vortex shedding for $-6^{\circ}$ wind angle of attack
$\multimap-R e=1.3 \times 10^{6} \longrightarrow-R e=1.7 \times 10^{6}$

Figure 15 shows the fluctuating lift coefficient due to vortex shedding, as a function of the reduced turbulence intensity for the $-6^{\circ}$ wind angle of attack. It can be seen that the $\dot{C}_{L}$ due to vortex shedding reduced linearly with the increase of the reduced turbulence intensity. The maximum difference in $\dot{C}_{L}$ for the tested range of reduced turbulence intensities was measured to be around $97 \%$. Also, it was noticed that $\dot{C}_{L}$ decreased with the $R e$ increase for the entire range of reduced turbulence intensities tested here.

\subsection{Effects of Turbulence Characteristics on Buffeting Loads}

In the bridge aerodynamics field, the spectral approach is a widely used method for the prediction of the buffeting response of the deck. When using this method, memory effects and self-excited forces can be explained reliably in the frequency domain. Memory effect is a term used to show that the bridge excitation at each time step $(t)$ depends on the interaction of the flow and the cross section at a time instant prior to that 
time step $(t-z)$. The memory effect can be described in the frequency domain using an aerodynamic admittance function. Self-exited forces are generated due to the structural motion in a wind flow and for a bridge deck with vertical and torsional degrees of freedom (DOFs), they can be computed from the following equations in frequency domain (Scanlan, 1978):

$$
\begin{aligned}
L_{h} & =\frac{1}{2} \rho U^{2} B\left[K H_{1}{ }^{*}(K) \frac{\dot{h}}{U}+K H_{2}{ }^{*}(K) \frac{B \dot{\alpha}}{U}+K^{2}{H_{3}}^{*}(K) \alpha+K^{2} H_{4}{ }^{*} \frac{h}{B}\right] \\
M_{\alpha} & =\frac{1}{2} \rho U^{2} B^{2}\left[K A_{1}{ }^{*}(K) \frac{\dot{h}}{U}+K A_{2}{ }^{*}(K) \frac{B \dot{\alpha}}{U}+K^{2} A_{3}{ }^{*}(K) \alpha+K^{2} A_{4}{ }^{*} \frac{h}{B}\right]
\end{aligned}
$$

where, $K$ is the reduced frequency $(2 \pi f B / U), f$ is the frequency of motion $(\mathrm{Hz})$, $h$ and $\alpha$ are the vertical and torsional displacements, over-dot indicates the derivatives with respect to time and $H_{i}{ }^{*}$ and $A_{i}{ }^{*}(i=1$ to 4$)$ are flutter derivatives.

Finding the buffeting loading from the spectral approach is based on quasi-steady theory. Therefore, wind-induced forces at each moment can be appreciated as forces generated by a steady wind having the same relative wind speed and direction as the momentary wind. From the quasi-steady theory, the buffeting loads can be calculated as:

$$
\begin{gathered}
F_{L, b}=1 / 2 \rho U B\left(2 C_{L} u+d C_{L} / d \alpha w\right) \\
M_{b}=1 / 2 \rho U B^{2}\left(2 C_{M} u+d C_{M} /_{d \alpha} w\right)
\end{gathered}
$$

where $F_{L, b}$, and $M_{b}$ are the buffeting loads in the vertical and torsional directions, $u$ and $w$ are fluctuating wind components in the along wind and perpendicular to mean wind directions. In the spectral approach, it is assumed that buffeting loading is a stationary random process. As a result, eq. (5) can be transformed into eq. (7) in the frequency domain: 


$$
S_{L}(f)=(1 / 2 \rho U B)^{2}\left(4 C_{L}^{2} S_{u}(f)|A(f)|^{2}+\left(d C_{L} / d \alpha\right)^{2} S_{w}(f)|A(f)|^{2}\right)
$$

where $|A(f)|^{2}$ is the aerodynamic admittance function, $S_{L}, S_{u}$ and $S_{w}$ are the power spectral densities of the lift per unit length, wind fluctuations in the along-wind and wind fluctuations perpendicular to wind, respectively. The application of aerodynamic admittance function was suggested by Davenport (1962) to resolve some of the quasi-steady assumption limitations by accounting for the lack of turbulence correlation spatially at higher frequencies. Larose et al (1998) showed the difference between the aerodynamic admittance function obtained for a single box girder bridge from direct measurement and a thin airfoil from empirical equations using Sears function. It was shown that the admittance function calculated from the Sears function was larger than the measured admittance, particularly at lower frequencies. The aerodynamic admittance function for the vertical direction can thus be calculated from the direct measurement using the following equation:

$$
|A(f)|^{2}=\frac{U^{2} S_{C_{L}}(f)}{4 C_{L}{ }^{2} S_{u}(f)+\left({ }^{d C_{L} / d \alpha}\right)^{2} S_{\omega}(f)}
$$

Eq. (7) gives the spectrum of the lift per unit length of the deck. In order to consider the span-wise distribution of the unsteady wind forces acting on the deck, the joint acceptance function is defined to transform point-like load to load on the entire span:

$$
S_{F_{L}}(f)=S_{L}(f)\left|J_{z}(f)\right|^{2}
$$

where $S_{F_{L}}$ is the power spectral density of the lift effective on the entire span and $\left|J_{z}(f)\right|^{2}$ is the joint acceptance function. Joint acceptance function measures the 
correlation between the spatial distributions of the forces across the span length. Based on the strip assumption, wind-induced loads on each strip are resulted only from the wind fluctuations on that strip. Therefore, it is assumed that spatial distribution of the windinduced loads across the span length follows the wind spatial distribution. However, Larose et al (1993) showed that wind generated loads were more correlated than the approaching wind, between any two assumed points across the span.

Figure 16 shows the comparison of the cross-sectional admittance for the different test setups at different angles of attack to Liepmann's approximation to the Sears function analytically derived for a thin airfoil with a lift slope of $2 \pi$ (Larose, 2002). The peaks observed for test results in this figure were due to the vortex shedding. It can be seen that for zero angle of attack, the measured admittances on the models were smaller than the predicted value based on the Liepmann's approximation for a thin airfoil at low reduced frequencies. However, at higher reduced frequencies the measured admittances on the models were larger than the approximated value for a thin airfoil. The difference in the higher frequency range can be attributed to the body induced turbulence for the twin-deck bridge section.

Based on the measured data, for a constant turbulence intensity, the crosssectional admittance was larger for the smaller model, corresponding to the larger $L_{u} / b$. It means that for the larger ratios of "length scale to bridge with", larger admittance was noticed. It can also be seen that, with changing the turbulence intensity from $3 \%$ to $6 \%$, the admittance function at lower reduced frequencies grew while at higher reduced frequencies, the turbulence intensity increase was accompanied by admittance reduction. In the lower reduced frequency range, it was noted that $L_{u} / b$ governed the admittance 
value; for different turbulence intensities but comparable values of $L_{u} / b$, almost similar admittance was calculated at lower reduced frequencies. For the lower reduced frequency range, the cross-sectional admittance showed an increasing trend with the $L_{u} / b$ increase.

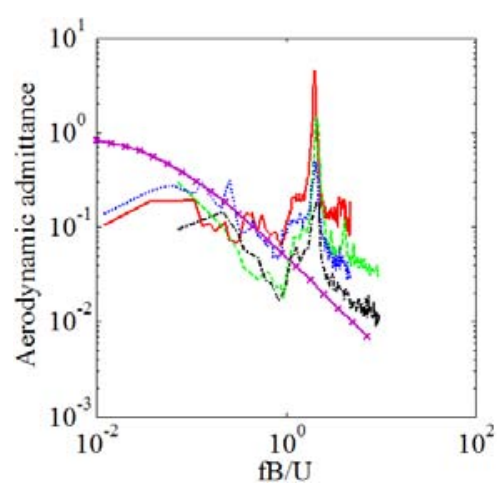

(a) Angle of attack $=-6^{\circ}$

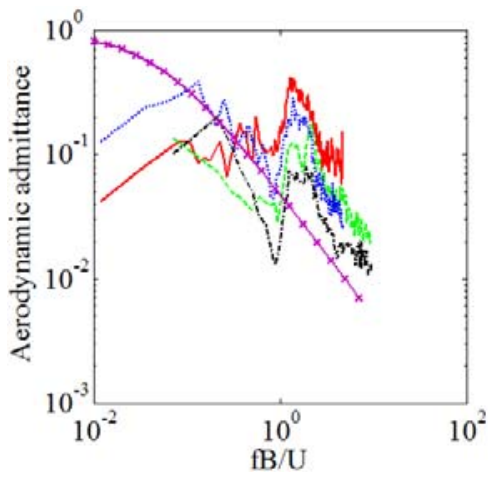

(b) Angle of attack $=0^{\circ}$

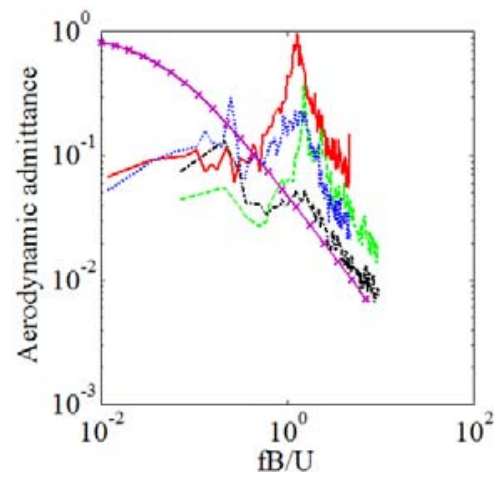

(c) Angle of attack $=+6^{\circ}$

Figure 16: Lift aerodynamic admittance vs. reduced frequency $(f B / U), R e=1.7 \times 10^{6}$

\footnotetext{
- Smooth flow-1/72 scale $\left(L_{u} / b=0.53\right)$----- Smooth flow-1/36 scale $\left(L_{u} / b=0.26\right)$

…….. Turbulent flow- $1 / 72$ scale $\left(L_{u} / b=1.18\right)$-..... Turbulent flow- $1 / 36$ scale $\left(L_{u} / b=0.59\right)$

$\rightarrow \leftarrow$ Liepmann's approximation to the Sears function
}

In Fig. 17, the span-wise cross-correlation coefficient was used to assess the spanwise distribution of the fluctuating wind forces acting on the deck. The span-wise crosscorrelation coefficient is defined as the co-variance of the fluctuating lifts measured at two strips separated by $\delta y$, divided by the product of the standard deviation of lift at each of these two strips. A total of six span-wise separations $(\delta y)$ were analyzed for the larger model $(1 / 36$ scaled model), varying from 0.2 to $1.26 \mathrm{~m}$. For the smaller model $(1 / 72$ scaled model), three different span-wise separations were considered, ranging from 0.42 to $1.04 \mathrm{~m}$. From Fig. 17, it can be seen that for a given wind exposure and a constant span-wise separation, a larger correlation coefficient was observed on the larger model compared to the smaller model. It was also noticed that for a given model $(1 / 36$ or $1 / 72$ 
scaled models), the span-wise correlation coefficient was larger in turbulent flow which had larger turbulence integral length scale and intensity. The first observation shows that the span-wise correlation of wind forces can be a factor of the model width rather than being only dependent to the oncoming flow characteristics. This contradicts the strip assumption based in which the span-wise correlation of wind fluctuations is assumed to be representative of the span-wise correlation of wind forces. The second observation was predictable, as it is expected that larger integral length scale should correspond to a larger span-wise correlation.

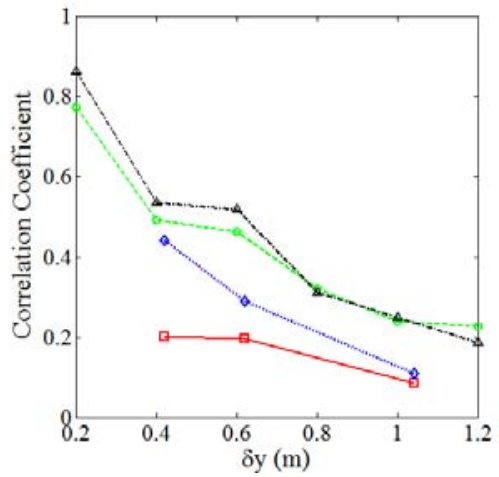

(a) Angle of attack $=-6^{\circ}$

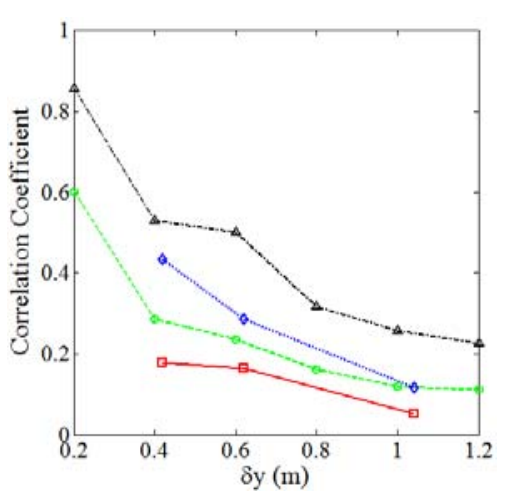

(b) Angle of attack $=0^{\circ}$

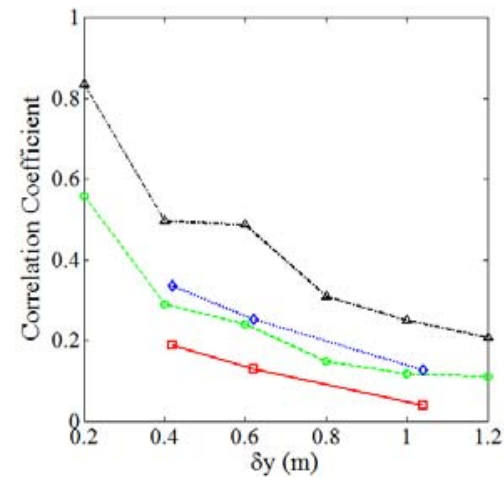

(c) Angle of attack $=+6^{\circ}$

Figure 17: Span-wise cross-correlation coefficient of lift force at $R e=1.7 \times 10^{6}$ —- Smooth flow-1/72 scale $\left(L_{u} / b=0.53\right)$-- - - . Smooth flow-1/36 scale $\left(L_{u} / b=0.26\right)$ $\cdots \cdots . . \cdots$ Turbulent flow-1/72 scale $\left(L_{u} / b=1.18\right) \cdots \cdots$ Turbulent flow- $1 / 36$ scale $\left(L_{u} / b=0.59\right)$

In order to better highlight the effect of $L_{u} / b$ ratio on the span-wise distribution of the fluctuating wind forces on a deck, the co-coherence of the lift forces from the different test setups are compared and shown for a given normalized separation $(\delta y / b)$ in Fig. 18. This satisfies the required geometric similarity regarding the separation length to ensure that any observed difference in the co-coherence is solely related to the $L_{u} / b$ 
effects. Co-coherence between two chord-wise strips can be obtained from the following equation:

$$
\operatorname{cocoh}(f, \delta y)=\frac{\operatorname{Co}_{12}(f)}{\sqrt{S_{L 1}(f) \times S_{L 2}(f)}}
$$

where $\mathrm{Co}_{12}$ is the co-spectrum between the fluctuating lifts at strips 1 and 2, and $S_{L}$ is the power spectral densities of the lift per unit length at each strip. From Fig.18, it can be seen that the co-coherence was dependent on the $L_{u} / b$ ratio, where larger $L_{u} / b$ corresponded to larger co-coherence. However, it can be seen that the turbulence intensity was as important as the turbulence integral length scale. From Fig. 18, it can be seen that there was a noticeable difference between the two cases of $L_{u} / b=0.53$ and 0.59 , while the ratios of the turbulence integral length scale to bridge width was almost constant. The observed difference was more pronounced at zero and $-6^{\circ}$ wind angles of attack while relatively similar curves were noticed for the $+6^{\circ}$ wind angle of attack.

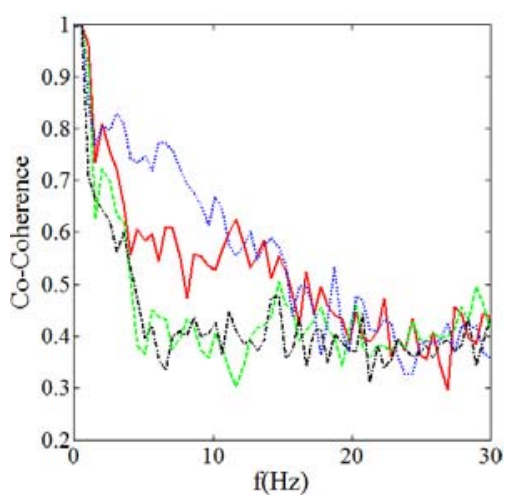

(a) Angle of attack $=-6^{\circ}$

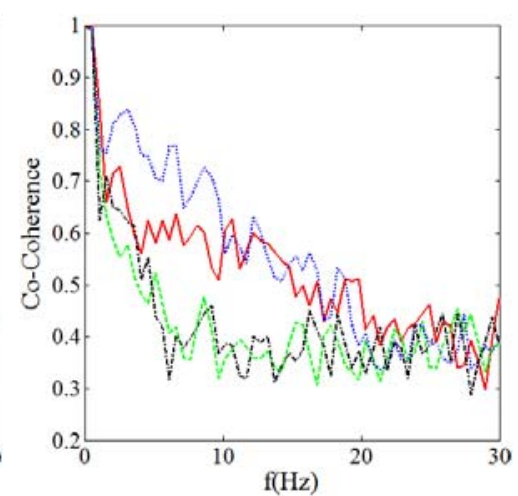

(b) Angle of attack $=0^{\circ}$

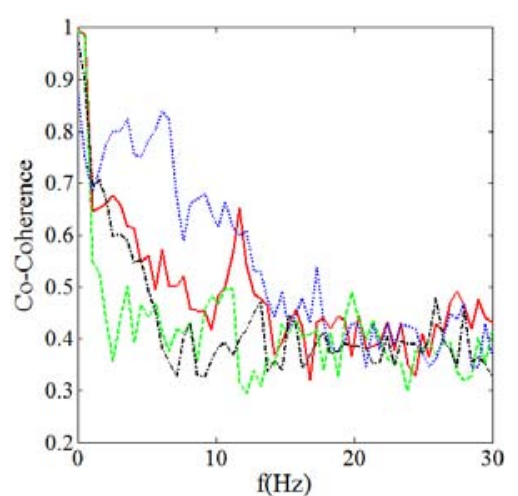

(c) Angle of attack $=+6^{\circ}$

Figure 18: Co-coherence of the lift forces for $\delta y / b=1.1$ at $R e=1.7 \times 10^{6}$ Smooth flow-1/72 scale $\left(L_{u} / b=0.53\right)$----- Smooth flow-1/36 scale $\left(L_{u} / b=0.26\right)$ Turbulent flow-1/72 scale $\left(L_{u} / b=1.18\right)$-...-.- Turbulent flow- $1 / 36$ scale $\left(L_{u} / b=0.59\right)$ 


\subsection{Conclusions}

In this paper, the effects of turbulence intensity and turbulence integral length scale on the aerodynamics of a twin-deck bridge were evaluated experimentally. Two models, at $1 / 36$ and $1 / 72$ scale, of the simplified cross section of the East-Bay bridge were tested statically under two flow conditions with nominal 3\% and $6 \%$ turbulence intensities. The effect of turbulence integral length scale for a given turbulence intensity was studied by comparing the results from the two different models. The ratio of the integral length scale to the bridge width on the smaller model was twice as of that on the larger model. The effect of turbulence characteristics on the chord-wise pressure distribution of the bridge deck was investigated. Also, from the spatial integration of the surface pressures along the chord, the fluctuating forces and moments were obtained at various span-wise locations. The fluctuating lift was further analyzed to investigate the turbulence effects on the vortex shedding and buffeting loads. The results can be summarized as follows:

1. With higher turbulence intensity, the peaks of the negative mean pressure distribution on the top surface of the upstream deck moved closer to the leading edge and the distribution became narrower on either side of the peak, for all the different wind angles of attack.

2. The effect of turbulence intensity on the mean pressure distribution on the bottom surfaces of the two decks was only noticed when the bridge was tilted to positive wind angles of attack. It was observed that higher turbulence intensity resulted in smaller mean negative pressures near the transition points at the leeward side of the 
upstream girder and the windward side of the downstream girder when the section was tilted to $+6^{\circ}$ wind angle of attack.

3. Comparing the results from the two different scale models in a given flow condition, it was noticed that the mean pressure distribution across the top surfaces was barely sensitive to the ratio of turbulence integral length scale to bridge width. However, it can be seen that a smaller ratio resulted in larger mean negative pressures near the transition points on the bottom surfaces. The observed difference in the mean pressure distribution across the bottom surfaces was more pronounce for positive angles of attack.

4. Force and moment coefficients were found to be more sensitive to the turbulence intensity compared to the turbulence integral length scale and Re. Based on the results, with the $R e$ increase, the negative mean lift coefficient increased in magnitude in both flow conditions. However, with the $R e$ increase, the mean drag coefficient showed a decreasing trend in smooth flow and an increasing trend in turbulent flow.

5. The steady force coefficients showed a linear relationship with the reduced turbulence intensity. Reduced turbulence intensity is a non-dimensional parameter which includes the simultaneous effects of turbulence intensity and turbulence integral length scale and is defined based on the partial turbulence simulation formulation. Based on the results, the mean drag coefficient showed a decreasing trend with the reduced turbulence intensity increase while the mean lift coefficient showed an increasing trend in magnitude. As a result, it is expected that testing at lower reduced turbulence intensity compared to the prototype would be conservative for mean drag 
coefficient estimation. However, for the mean lift coefficient, testing at lower reduced turbulence intensity would result in smaller values than that the prototype would experience. For the given range of reduced turbulence intensities tested here, the mean drag changed around $34 \%$.

6. Based on the results, the sensitivity to Re was higher for the larger reduced turbulence intensities.

7. Analysis of the fluctuating lift coefficient spectra indicated that larger turbulence intensity had a mitigating effect on the vortex shedding. Also, larger turbulence integral length scale to bridge width ratios resulted in larger fluctuating lift coefficient due to vortex shedding. The fluctuating lift coefficient due to vortex shedding reduced with the reduced turbulence intensity increase. It was noticed that vortex shedding induced $C_{L}$ decreased with the $R e$ increase for the entire range of reduced turbulence intensities tested here.

8. The aerodynamic admittances, obtained from the lift and wind components spectra, were larger for the larger turbulence integral length scale to bridge width ratios. It was also observed that the approximated aerodynamic admittance from empirical equations for a thin airfoil body was different from the direct measured value on a twin-deck bridge. While the approximated value was larger at lower reduced frequencies, the measured aerodynamic admittance showed to be larger than the approximated value at larger reduced frequencies.

9. Based on the strip method, it is expected that the span-wise distribution of the wind generated loads would be only a factor of the span-wise distribution of the approaching wind speed. However, results showed that the cross correlation of the 
wind-induced loads was also a function of the model scale. Analysis of the span-wise distribution of the wind loading showed that for a given separation length, the cross correlation of the wind loads was larger for the larger model. This shows the limitations associated with the strip assumption in which the spatial distribution of the wind speed components is considered to be representative of the spatial distribution of the generated wind loading.

\subsection{Acknowledgements}

This research was supported by the National Science Foundation (NSF) [NSF MRI Award CMMI-0923365] for acquisition of instruments and Florida Center of Excellence in Hurricane Damage Mitigation and Product Development. The authors would like to acknowledge Maryam Asghari Mooneghi, Walter Conklin and Roy Liu Marques for their unreserved input during testing at the Wall of Wind. The findings reported in this article are those of the authors alone, and do not necessarily represent the views of sponsoring agencies. We also acknowledge the contribution of T.Y. Lin International in providing us with information on the East Bay Bridge. The results provided in the paper do not extrapolate directly to the East Bay Bridge as the geometry was simplified for the purposes of the present research.

\subsection{References}

Blevins, R.D., 2001. Flow-induced Vibration. Krieger Publishing Company.

Davenport, A.G., 1962. Buffeting of a suspension bridge by storm winds. Journal of Structural Engineering 88, 233-270.

Frandsen, J.B., 2001. Simultaneous pressures and accelerations measured full-scale on the Great Belt East suspension bridge. Journal of Wind Engineering and Industrial Aerodynamics 89, 95-129. 
Gu, M., Zhang, R., Xiang, H., 2001. Parametric study on flutter derivatives of bridge decks. Engineering Structures 23, 1607-1613.

Haan Jr., F.L., Kareem, A., Szewczyk, A.A., 1998. The effects of turbulence on the pressure distribution around a rectangular prism. Journal of Wind Engineering and Industrial Aerodynamics 77-78, 381-392.

Huston, D.R., 1986. The Effects of Upstream Gusting on the Aeroelastic Behavior of Long Suspended-Span Bridges. Princeton University.

Irwin, P., Cooper, K., Girard, R., 1979. Correction of distortion effects caused by tubing systems in measurements of fluctuating pressures. Journal of Wind Engineering and Industrial Aerodynamics 5, 93-107.

Irwin, P.A., 1977. Wind Tunnel and Analytical Investigations of the Response of Lions Gate Bridge To Turbulent Wind. National Research Council of Canada, National Research Council of Canada, p. 62.

Irwin, P.A., 1998. The role of wind tunnel modeling in the prediction of wind effects on bridges, in: Esdahl, A.L.S. (Ed.), The international symposium on advances in bridge aerodynamics, Copenhagen, Denmark, pp. 99-117.

Irwin, P.A., 2004. Bluff body aerodynamics in wind engineering, 5th International Colloquium on Bluff Body Aerodynamics and Applications (BBAA V). Elsevier, Ottawa, Canada, pp. 51-57.

Katsuchi, H., Yamada, H., 2011. Study on turbulence partial simulation for wind-tunnel testing of bridge deck, in: Proceedings of the 13th International Conference on Wind Engineering.

Kwok, K.C.S., Qin, X.R., Fok, C.H., Hitchcock, P.A., 2012. Wind-induced pressures around a sectional twin-deck bridge model: Effects of gap-width on the aerodynamic forces and vortex shedding mechanisms. Journal of Wind Engineering and Industrial Aerodynamics 110, 50-61.

Larose, G.L., 2002. The dynamic action of gusty winds on long-span bridges, Department of Structural Engineering and Materials. Technical University of Denmark, p. 179.

Larose, G.L., Davenport, A.G., King, J.P.C., 1993. On the Unsteady Aerodynamic Forces on a Bridge Deck in Turbulent Boundary Layer Flow, 7th U.S. National Conference on Wind Engineering, California, USA.

Larose, G.L., Tanaka, H., Gimsing, N.J., Dyrbye, C., 1998. Direct measurements of budeting wind forces on bridge decks. Journal of Wind Engineering and Industrial Aerodynamics 74-76, 809-818. 
Larsen, A., Esdahl, S., Andersen, J.E., Vejrum, T., 2000. Storebælt suspension bridge vortex shedding excitation and mitigation by guide vanes. Journal of Wind Engineering and Industrial Aerodynamics 88, 283-296.

Li, Q.S., Melbourne, W.H., 1995. An experimental investigation of the effects of freestream turbulence on streamwise surface pressures in separated and reattaching flows. Journal of Wind Engineering and Industrial Aerodynamics 54-55, 313-323.

Matsumoto, M., Shiraishi, N., Shirato, H., Stoyanoff, S., Yagi, T., 1993. Mechanism of, and turbulence effect on vortex-induced oscillations for bridge box girders. Journal of Wind Engineering and Industrial Aerodynamics 49, 467-476.

Scanlan, R.H., 1978. The action of flexible bridges under wind, I: Flutter theory Journal of Sound and Vibration 60, 187-199.

Scanlan, R.H., Lin, W.H., 1978. Effects of turbulence on bridge deck flutter derivatives. Journal of the Engineering Mechanics Division 104, 719-733.

Wardlaw, R.L., Tanaka, H., Utsunomiya, H., 1983. Wind tunnel experiments on the effects of turbulence on the aerodynamic behaviour of bridge road decks. Journal of Wind Engineering and Industrial Aerodynamics 14, 247-257. 


\section{CHAPTER IV \\ EFFECTS OF DECK DETAILS ON THE TWIN DECK BRIDGE AERODYNAMICS}

(A paper submitted to the journal of Wind and Structures) 


\title{
CHAPTER IV
}

\section{EFFECTS OF DECK DETAILS ON THE TWIN DECK BRIDGE AERODYNAMICS}

\author{
Ramtin Kargarmoakhar ${ }^{a},{ }^{*}$ Arindam Gan Chowdhury ${ }^{b}$, Peter Irwin ${ }^{c}$
}

\subsection{Abstract}

The significant concern in the design of twin deck bridges is centered on vortex induced excitations. In this paper, the effects of deck details, such as gap width, traffic barriers and bike path on the aerodynamic characteristics of a twin deck bridge, particularly on the vortex shedding, were investigated. In order to evaluate the effects of gap width, models with three different gap widths were tested in static condition. The selected gap widths where chosen within a reasonable range of values proposed in the past literature based on desirable aerodynamic performance and practical construction issues. The effect of deck appurtenances was also studied by including the traffic barriers, bike path and counterweight member in the simulation for the smallest gap width. In all tests, the models were equipped with the pressure taps that were distributed along the chord length. The wind induced loads were also measured directly by load cells. Results showed that changing the gap width and including the traffic barriers modified the pressure distribution around the section, indicating a change in the flow pattern and consequently a different aerodynamic behavior. However, the pressure distribution was

${ }^{a} \mathrm{PhD}$ candidate, Civil and Environmental Engineering/International Hurricane Research Center, Florida International University, Miami, FL, USA, rkarg001@,fiu.edu

b Corresponding Author, Associate Professor, Civil and Environmental Engineering/International Hurricane Research Center, Florida International University, Miami, FL, USA, chowdhur@fiu.edu, Tel: (305) 348-0518

${ }^{c}$ Professor of Practice, Civil and Environmental Engineering/International Hurricane Research Center, Florida International University, Miami, FL, USA, peairwi@fiu.edu 
less sensitive to the inclusion of the bike path. Among all the studied deck detail parameters, force and moment coefficients showed the highest sensitivity to the inclusion of the traffic barriers. Based on the results, sections equipped with the traffic barriers are susceptible to vortex shedding and the frequency of the vortex shedding increased with the gap width increase.

Keywords: Twin-deck bridge; Gap width; Traffic barriers; Deck appurtenances; Vortex shedding; Force and moment coefficients; Aerodynamic response.

\subsection{Introduction}

Wind loading is a major concern in the design of long span bridges due to their inherent low stiffness and damping compared with medium and small span bridges. Wind-structure interaction can create different response phenomena which may lead to stability problems. Flutter is an aeroelastic instability in which the aerodynamic forces get large enough to modify the oscillating frequencies of the structure and cancel out the mechanical damping effects, leading to the structure failure. Flutter wind speed is therefore a major concern in limiting the maximum length to which a bridge can span for a given site. Increasing the span length has become viable by introducing multiple deck cross sections, as it was shown by many researches that multiple deck bridges had superior stability against flutter compared with single deck sections (Sato et al, 2002). The downside of such cross sectional shape adoption is that multiple deck bridges suffered from vortex-induced vibration (VIV) in many cases (Larsen et al, 2008; Li et al, 2011). VIV happens when the vortex shedding frequency of flow separating around the structure is close to one of the structural oscillation frequencies which can create large but self-limited amplitude motions. Replacement of old bridges with new bridges can 
lead to the design of two bridges alongside each other, with an opening between the two. Similar to multiple deck bridges, placement of a new bride next to an older bridge can end up in two or more decks which can interact aerodynamically and get influenced by each other while being independent structurally.

Previous literature suggests that modifying the cross sectional shape, even by changing minor details, can change the aerodynamic performance of the section considerably (Bruno and Mancini, 2002; Fransos and Bruno, 2010; Jones et al, 1995; Kwok et al, 2012; Nagao et al, 1997). One factor which has been shown to have significant influence is the gap width. Yung and GE (2008) studied the effect of gap width on the flutter stability of the "Xihoumen bridge" with a twin trapezoidal deck cross section and explained that the flutter onset wind speed increased with the increase of the gap width up to a certain point above which the onset wind speed tended to decrease with the additional increase of the gap width. Based on their results, the optimum gap width to improve the flutter stability was measured to be equal to 1.2 times the single deck width. However, due to cost considerations, the ratio of the gap width to the single deck width is normally set to be smaller than 1.0 (Trein et al, 2013). Keeping in mind that flutter stability improves with the gap width increase, it is likely that the vortex shedding mechanism is also affected by the change of the gap width.

The effect of railings on the bridge aerodynamics has been evaluated previously (Bruno and Mancini, 2002; Jones et al, 1995; Larsen et al, 2008; Nagao et al, 1997). Due to the small size of the railings compared to the bridge deck, it was suspected that the effect of railings simulation in wind studies could be minimal and limited to local effects on the deck. However, it turned out that the presence and even details of the railings had 
significant effects on the stability against flutter (Jones et al, 1995) as well as vortex shedding (Nagao et al, 1997), pressure distribution and integrated forces (Bruno and Mancini, 2002) for the single deck bridges. Studying the section without the barriers also gives an insight on the bridge aerodynamic behavior during construction. Usually the deck is built first and barriers are added afterwards. It is therefore of interest to evaluate the effect of deck appurtenances such as traffic barriers and attached bike path on the aerodynamic behavior of twin deck bridges to see how these details can change the flow pattern around the section and whether they improve or worsen the vortex shedding.

In fact the available information on how changes in the gap width can alter the vortex shedding mechanism on a twin deck bridge is limited. Also, according to the author's knowledge, there are only few studies in the literature evaluating the effect of traffic barriers and bike path on the twin deck bridges aerodynamics. This study evaluates the effect of deck details, namely the gap width, traffic barriers and bike path on the aerodynamic behavior of a twin deck bridge. The outcome of this study can benefit the design practice by: (1) providing information on the bridge aerodynamic response for the condition that traffic barriers are not in place as observed in the construction stage, (2) defining how adding the bike path mitigates or improves vortex shedding and changes wind induced loads, and (3) quantifying the gap width effect on the wind induced loads and vortex shedding mechanism for a twin deck bridge equipped with traffic barriers so that the gap opening can be optimized.

To this end, the effect of the aforementioned shape details were investigated on the wind generated pressure distribution, resulting forces, and force power spectra, the last of which can shed light on the vortex shedding mechanism. In order to study the 
effect of gap width, 1:72 scaled models of a twin deck bridge with gap to single deck width ratios of $0.5,0.75$ and 1.0 were tested under a relatively smooth flow condition with turbulence intensity in the order of 3\%. The gap ratios were chosen such that they represented the most common cases that are used in reality. A larger, 1:36 scaled model of the same cross section was tested for three cases: (i) bare deck, (ii) deck equipped with the traffic barriers, (iii) and deck equipped with the bike path attached to the upwind side and the corresponding counterweight member attached to the downwind side. These tests, performed under a more turbulent flow with turbulence intensity close to $7 \%$, were conducted to evaluate the effect of deck appurtenances. The 12-fan Wall of Wind (WoW) open jet facility at Florida International University (FIU) was used for all the experiments conducted for the present study. Tests were performed statically and the models were equipped with pressure taps that were distributed along the chord length. The pressure distribution and the resulting effective forces on individual decks helped to clarify the flow characteristics around the section and the vortex shedding mechanism for the two decks. The effective forces and pitching moment were measured by integration of the measured pressures and also directly by load cells installed at the model supports. The results from this study can be extended and used for the case of two parallel bridge decks next to each other with independent structures that can interact aerodynamically, such as the case of building a new bridge parallel to an older bridge deck.

\subsection{Description of the Experimental Setup and Testing Procedure}

\subsection{1 $\quad$ Twin deck bridge}

The importance of the deck appurtenances (traffic barriers, bike path, and counterweight member) and configuration (gap width) on the aerodynamic response of a 
twin deck bridge was evaluated in this study. The cross section of the "San FranciscoOakland" twin deck bridge ("East Bay" bridge) was chosen for this study as preliminary studies on the bare deck configuration of this bridge showed that this section was susceptible to vortex shedding (Kargarmoakhar et al, 2015). The bare section of the "East Bay" bridge consists of two trapezoidal girder decks connected by a system of transverse cross beams. Each deck has a width to depth ratio of 5. Trapezoidal girder bridges with relatively similar aspect ratio have shown to be prone to vortex shedding (Schewe and Larsen, 1998).

East span of the San Francisco-Oakland bay bridge is an asymmetric selfanchored suspension bridge with a main span of $385 \mathrm{~m}$ and a back span of $185 \mathrm{~m}$ (see Fig. 1 (a)). The two decks are linked together with $10 \mathrm{~m}$ wide transverse beams every $20 \mathrm{~m}$. The single deck chord length $(C)$, total width $(B)$, gap width (b) and height $(H)$ of the bridge deck are $28 \mathrm{~m}, 71 \mathrm{~m}, 14 \mathrm{~m}$ and $5.5 \mathrm{~m}$, respectively (see Fig. 1 (b)). The counterweight member was included in the original design to counterbalance the bike path weight attached to the section (see Fig. 1 (c)). The information on the bridge specifications was kindly provided by T.Y. LIN INTERNATIONAL GROUP which was involved in all phases of developing this bridge, from design to construction. 


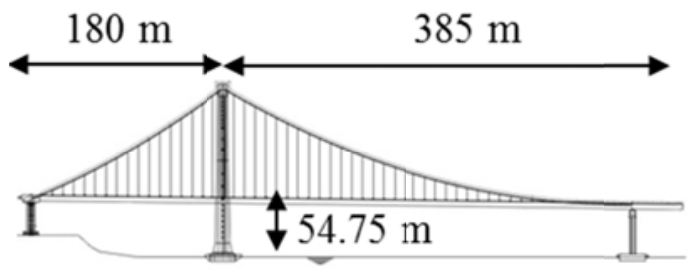

(a) General layout

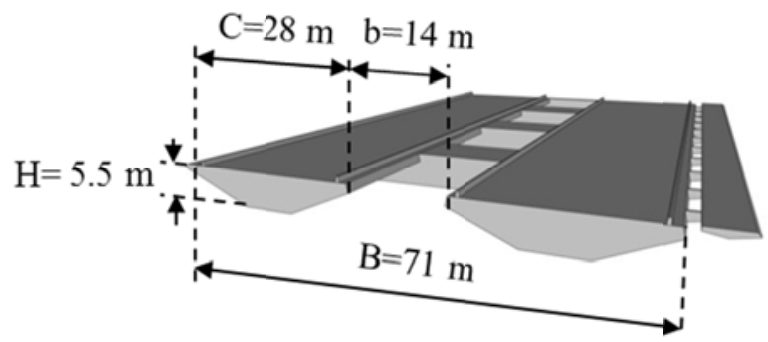

(b) Bridge dimensions

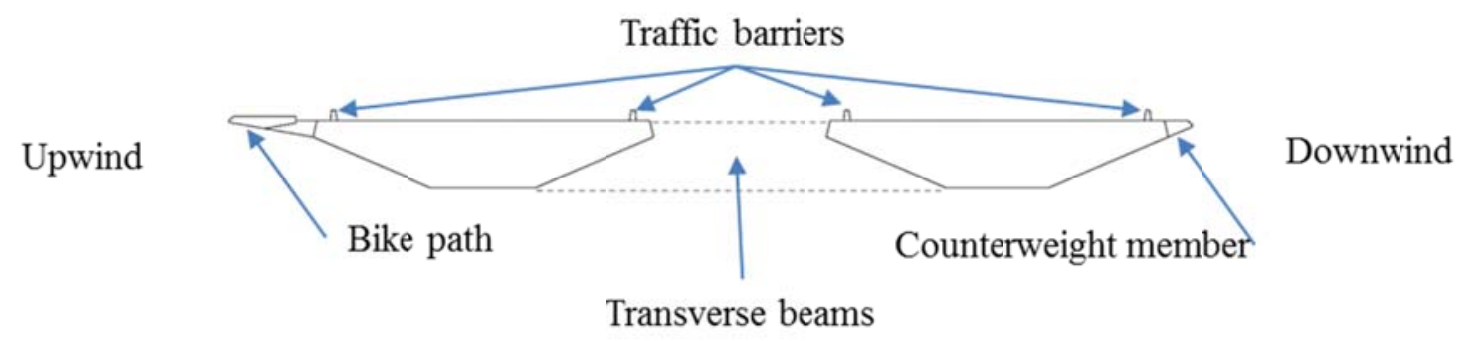

(c) Cross sectional shape

Figure 1: East span of the San Francisco-Oakland bay bridge

\subsubsection{Test setup}

In order to investigate the sensitivity of the aerodynamic response to shape details, the bridge response was measured and calculated in form of surface pressures and force/moment coefficients. Gap width and deck appurtenances were the variables that were changed for different tests. For each test, a pressure tapped rigid model of the bridge was made out of a wooden frame and was covered by Plexiglas plates to reproduce the cross sectional shape and provide room for the pressure tubes inside the model. During the test, two large streamlined end walls covered the two ends of the model to reduce the end effects and provide a 2-D wind flow. Figure 2 shows a general view of the test setup in front of the WOW. 
A Scanivalve Corporation pressure scanning system was used to measure pressures at a sampling frequency of $512 \mathrm{~Hz}$ for a period of two minutes for each test case. In order to reduce the pressure tube length, pressure scanners were placed at the end of the model on each side and each tap was connected to the pressure scanning system in the shortest distance. A transfer function designed for the tubing (IRWIN ET AL, 1979) was used to correct for the tubing effects. In each test, the model was supported by an aluminum I-beam on each side that was connected to the model with three aluminum pipes. I-beams were placed inside the side walls and were installed on top of a pair of JR3 multi-axis load cells to directly measure the forces (Drag and Lift) and the pitching moment generated by the wind. All the data from force scanners were recorded using a CompactRIO data acquisition system at $100 \mathrm{~Hz}$ sampling rate. Both systems, load cells and pressure scanners, were used to facilitate comparison of the wind effects.

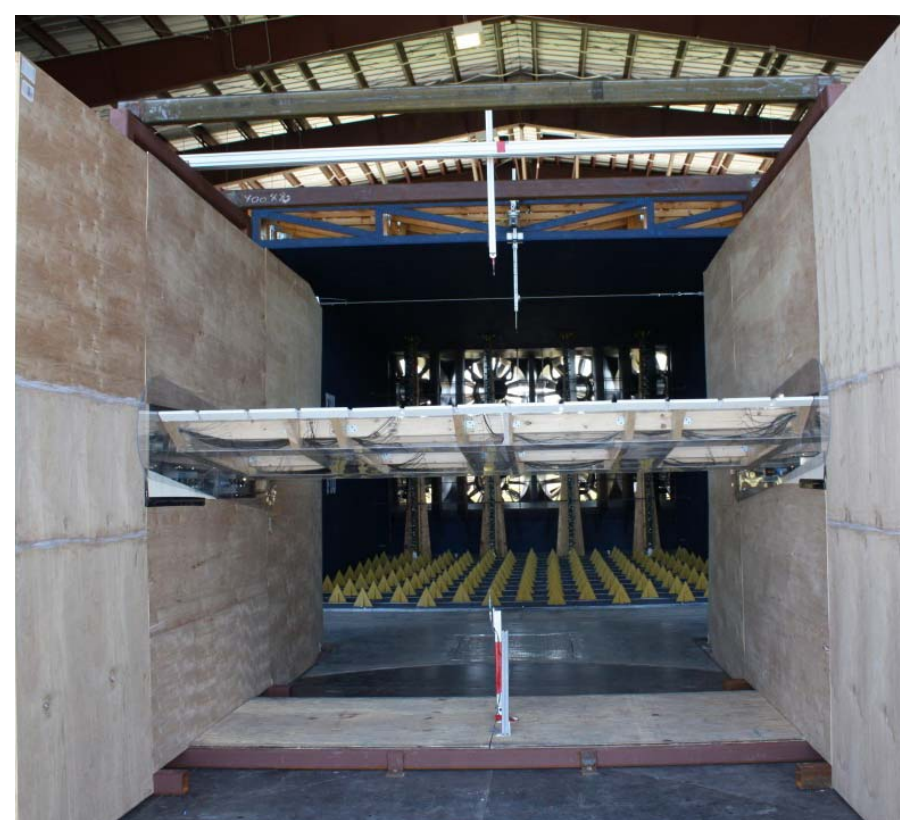

Figure 2: Test setup in front of the Wall of Wind 


\subsubsection{Model setup for the gap width study}

In order to study the effects of gap width, 1:72 scaled models with three different gap to single deck width ratios $(\mathrm{b} / \mathrm{C})$ were tested in the WOW facility. Gap width variation represented a common range of gap widths that is normally assumed in the design of twin deck bridges, based on the effectiveness and economy. Three gap to single deck width ratios of $0.5,0.75$ and 1.0 were considered for this study, as shown in Fig. 3 . The bike path that exists on the real bridge was not included in these tests as it would have added to the complexity of the interpretation of the results with regards to the gap width effects. Pressures were measured at a total of 104 to 108 points, depending on the gap width, distributed around four strips that were located along the span as shown in Fig. 4(a). A sectional view of bridge models and the distribution of pressure taps are shown in Fig. 5(a). The length of the model (L) was $3.12 \mathrm{~m}$ and the width varied from $0.97 \mathrm{~m}$ to $1.16 \mathrm{~m}$, depending on the gap width. Models with different gap widths were tested at five wind angles of attack, including $-6^{\circ},-3^{\circ}, 0^{\circ},+3^{\circ}$, and $+6^{\circ}$. Positive angle was defined as the one which moved the bridge leading edge upward (see Fig. 5).

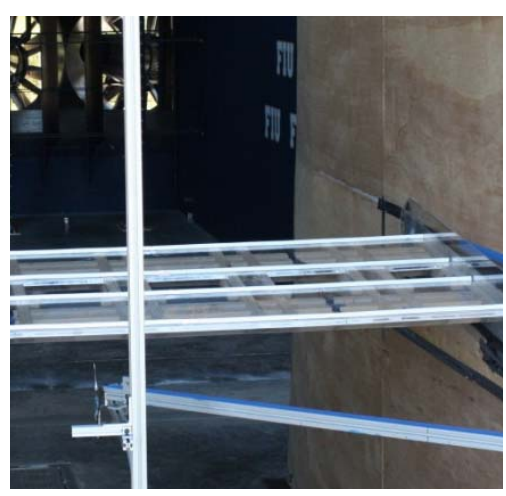

(a) $\mathrm{b} / \mathrm{C}=0.5$

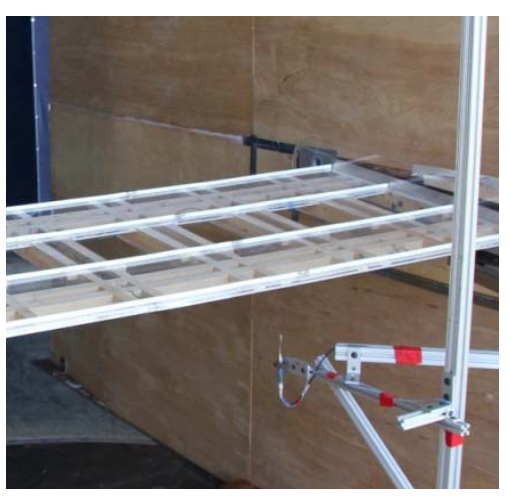

(b) $\mathrm{b} / \mathrm{C}=0.75$

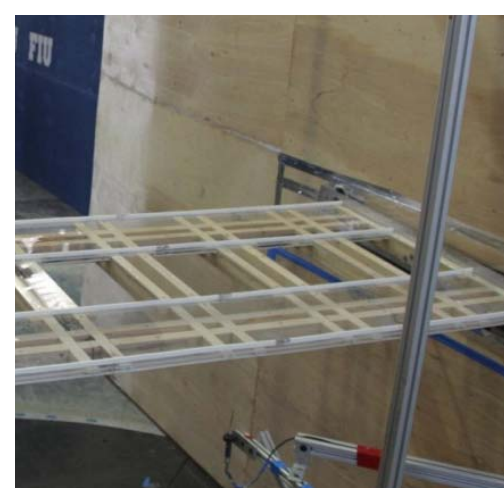

(c) $b / C=1.0$

Figure 3: Test setup for studying the gap width effects 


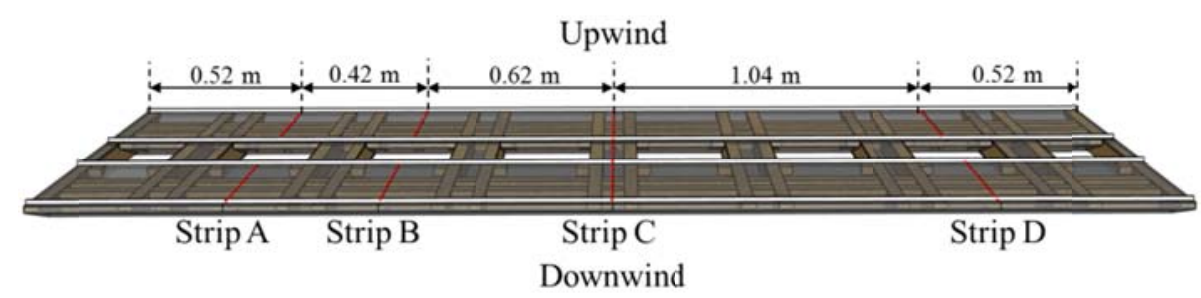

(a) Model setup for the gap width study

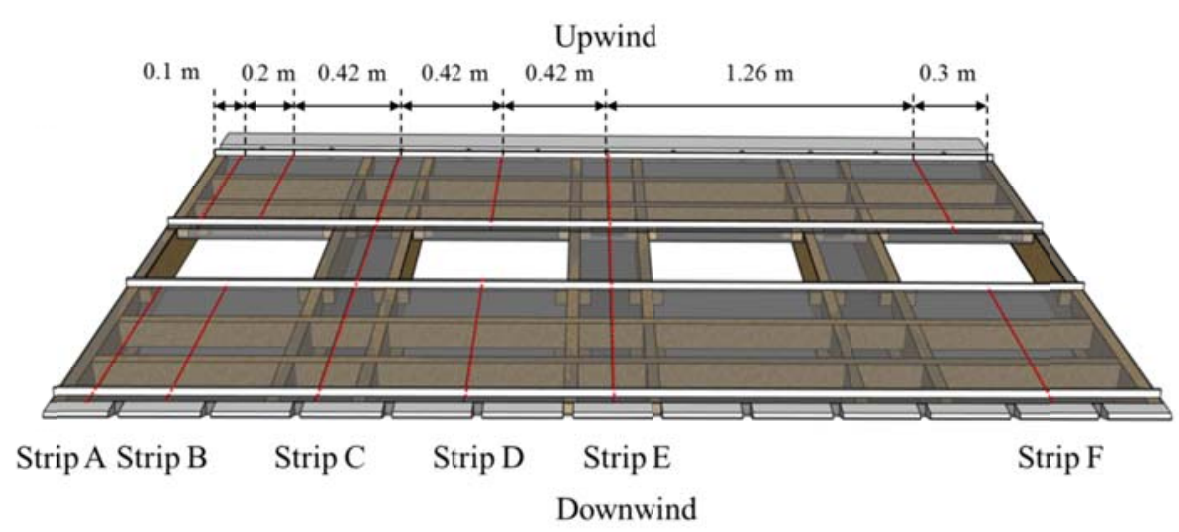

(b) Model setup for the deck appurtenances study

Figure 4: Distribution of strips of pressure taps

\subsubsection{Model setup for the deck appurtenances study}

A 1:36 scaled model of the bridge was built and tested with different deck equipment to study the effects of deck appurtenances on the wind induced responses. Three deck configurations were considered for this purpose. The first case was the condition with no appurtenance attached to the bridge and was called the bare condition (see Fig. 6 (a)). The second case considered was the section equipped with traffic barriers on the top surface with no bike path or counterweight member attached to the model (see Fig. 6 (b)). In the third case, the bike path was attached to the upwind side of the model and the counterweight member was attached to the downwind side without the inclusion 
of the traffic barriers (see Fig. 6 (c)). The model width and length were $1.94 \mathrm{~m}$ and 3.12 $\mathrm{m}$, respectively. Thus the span to width ratio was 1.6 , which is similar to the ratio used in a previous research by (Laima et al, 2013) to study the VIV of twin-girder bridges. Also, Piña and Caracoglia (2009) studied flutter derivatives for Carquinez Strait Bridge using two models with different span to width ratios and suggested that span to width ratio of 1.7 was adequate for 2-D experiments. For this set of tests, pressures were measured at a total of 280 points distributed around six strips as shown in Figs. 4(b) and 5(b). Tests were performed for $-3^{\circ}, 0^{\circ}$ and $+3^{\circ}$ wind angles of attack.

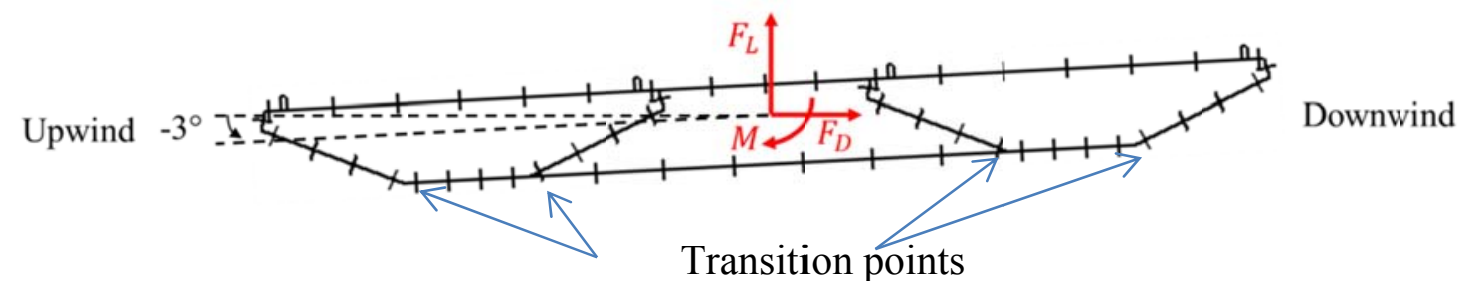

(a) Model setup for the gap width study (model is tilted to $-3^{\circ}$ angle of attack)

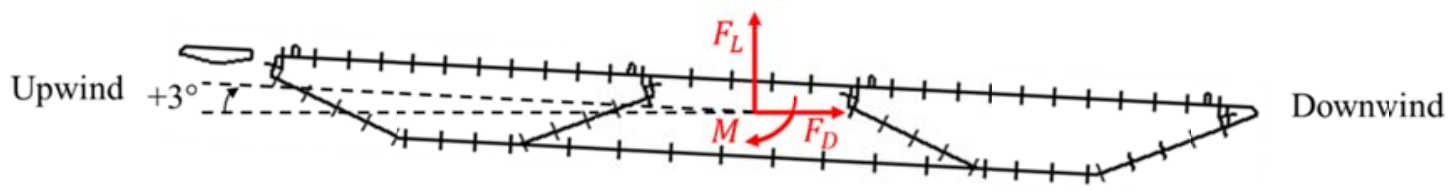

(b) Model setup for the deck appurtenances study (model is tilted to $+3^{\circ}$ angle of attack)

Figure 5: Positions of the pressure taps 


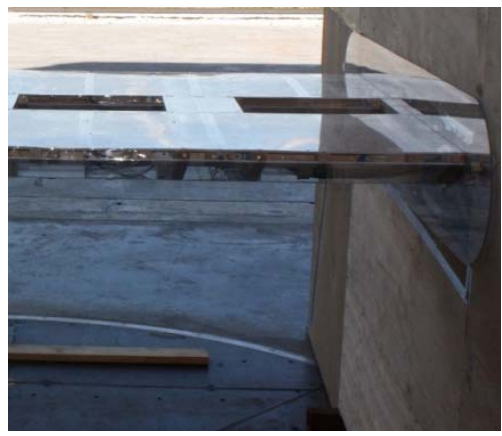

(a) Bare deck

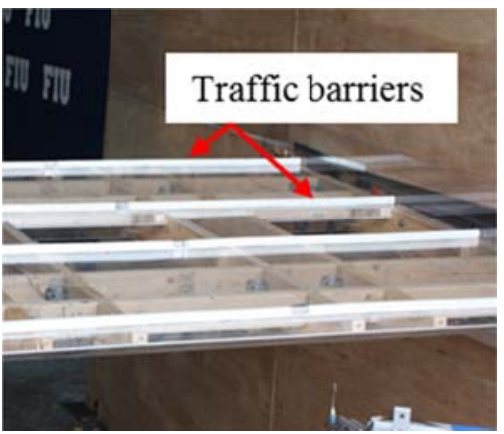

(b) Bare deck + traffic barriers

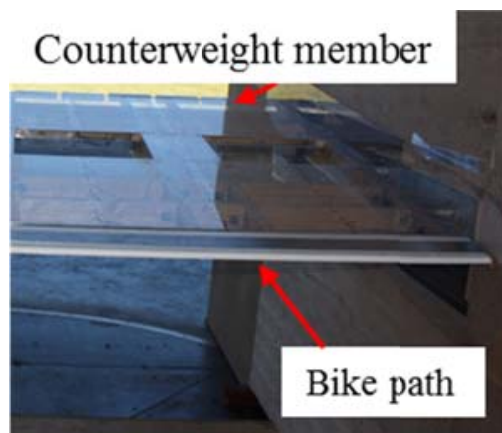

(c) Bare deck + bike path + counterweight member

Figure 6: Test setup for studying the effects of deck appurtenances

\subsubsection{Wind flow characteristic}

In order to measure the wind speeds, two cobra probes were placed at $0.9 \mathrm{~m}$ below and above the middle point of the section during the WOW tests to minimize the local effects of the model on the measured wind speed. The average of the measured wind speeds at these two points was used as the mean wind speed in the calculation of the pressure, force and moment coefficients. For the free wind speed measurement, three cobra probes were installed at bridge height along the bridge center line and were spaced equally from each other and from the end walls while the model was not in place (see Fig. 7(a)). The ratio between the measured wind speed at bridge height and the average of the two points at $0.9 \mathrm{~m}$ distant was calculated for the free flow condition. In order to adjust the wind speed for the test condition, the ratio obtained from the free flow condition was squared and applied to the pressure, force and moment coefficients. Data measured from the Cobra probes were collected at $100 \mathrm{~Hz}$ using the same CompactRIO data acquisition system that was used for force measurements. 
Wind tunnel tests on bridges are often performed in flows with minimal turbulence as such flow produces conservative results (Gu et al, 2001; Wu and Kareem, 2012). For the first part of the study which focused on the effects of the gap width, tests were performed in a nominally smooth flow. Turbulence intensity (TI) and turbulence integral length scale $\left(L_{u}^{x}\right)$ were measured to be $3 \%$ and $0.2 \mathrm{~m}$ for the along wind direction, and $2.8 \%$ and $0.12 \mathrm{~m}$ for the across wind direction, respectively for this case. The test wind speed for the gap width effect study was set to $27.3 \mathrm{~m} / \mathrm{s}$ which corresponded to a Reynolds number $(R e)$ of $1.4 \times 10^{5}$ based on the section's depth.

In order to generate turbulence and boundary layer characteristics, a set of triangular spires and floor roughness elements were put in front of the fans exit. Tests for the deck appurtenances effects were performed in a wind field with turbulence intensity and turbulence integral length scale of $7 \%$ and $0.45 \mathrm{~m}$ for the along wind direction, and $6.3 \%$ and $0.22 \mathrm{~m}$ for the across wind direction, respectively. For the deck appurtenances studies, the sectional model was tested under higher wind speeds, varying from $9.8 \mathrm{~m} / \mathrm{s}$ to $38.9 \mathrm{~m} / \mathrm{s}$ representing a range of $1.0 \times 10^{5}$ to $4.0 \times 10^{5}$ for $R e$, based on the section's depth. Figure 7(b) shows the comparison of the wind power spectral density in the along wind direction for the two test conditions. The 12000 data points measured at each probe from 2 minutes of testing at $100 \mathrm{~Hz}$ was divided into 11 blocks, each containing 1024 points. Power spectral density was calculated for each block and the average of the 11 blocks was used as the representative wind spectra. 


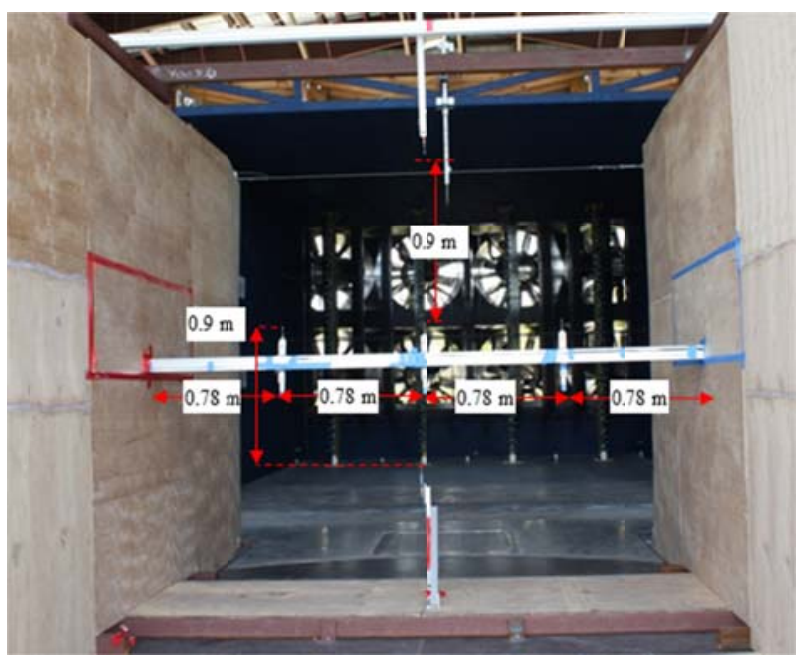

(a) Cobra probe position

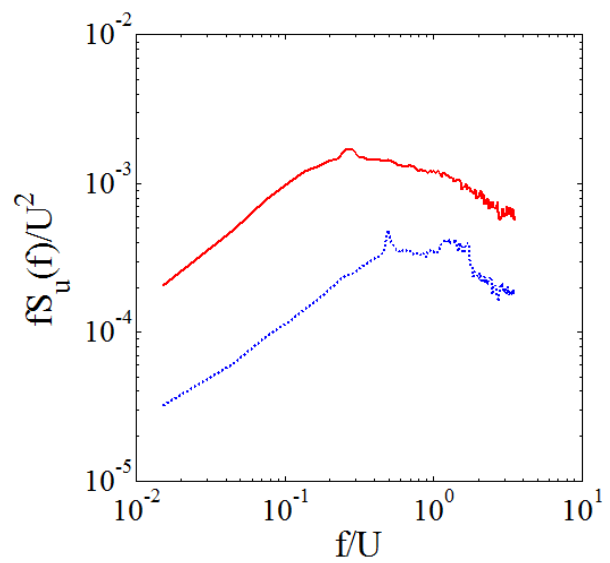

(b) Normalized power spectral density (PSD) of wind speed -..- $\mathrm{TI}=3 \%$ - $\mathrm{TI}=7 \%$

Figure 7: Wind speed measurement

\subsection{Result and Discussion}

In this paper, the effect of deck details (i.e. the gap width, traffic barriers and bike path) were evaluated on the mean and fluctuating pressure distribution, force/moment coefficients and vortex shedding mechanism. Pressure data collected around the cross section were used to understand the pressure distribution and depict the local effects of the flow modification on the mean and fluctuating pressures. Overall force/moment coefficients were obtained by integrating the pressures on the surface and were compared to the collected data from the load cells. The steady and fluctuating force/moment coefficients were helpful in evaluating the overall aerodynamic response of the bridge in terms of stability and vortex shedding.

\subsection{1 $\quad$ Pressure Distribution}

Literature suggests that pressure distribution can be used to identify separation and reattachment zones around bluff structures under wind flow (Kwok et al, 2012; Li 
and Melbourne, 1995). It is believed that highest suctions occur at the forward part of the separation bubble, with a large negative mean pressure being a sign of a separation region. Fluctuating pressure shows the effect of turbulence in oncoming wind or body induced turbulence and maximum fluctuating pressures correspond to reattachment zones. Knowledge of the separation and reattachment zones can be used to understand the source and mechanism of the excitation due to the wind flow and therefore can be used to generate ideas for the mitigation of wind generated excitations. In this paper, mean and fluctuating pressure distributions were considered in order to highlight the changes in the structure of the flow due to the modification of the deck details. Pressure distributions are reported in terms of non-dimensional mean and RMS (root mean square) pressure coefficients $\left(C_{\bar{P}}\right.$ and $C_{\sigma_{P}}$, respectively) obtained as:

$$
C_{\bar{P}}=\frac{P_{a v g}}{1 / 2 \rho U^{2}}, C_{\sigma_{P}}=\frac{\sigma_{P}}{1 / 2 \rho U^{2}}
$$

where: $P_{a v g}$ is the mean pressure $\left(\mathrm{N} / \mathrm{m}^{2}\right)$ obtained from the pressure time history data at each tap, $\sigma_{P}$ is the standard deviation of pressure time history at each tap $\left(\mathrm{N} / \mathrm{m}^{2}\right)$, $\rho$ is air density $\left(\mathrm{kg} / \mathrm{m}^{3}\right)$, and $U$ is the mean wind speed $(\mathrm{m} / \mathrm{s})$ at the model height. In the following sections, for the mean pressure distribution figures, negative pressures were plotted outside the cross section and positive pressures were plotted inside it.

\subsubsection{Effects of gap width}

Figures 8 and 9 show the effect of the gap width on the mean and fluctuating pressure distribution for different wind angles of attack. All the mean and fluctuating pressure distributions shown for the gap width effects are based on the measured data at the strip B location. From the mean pressure distribution figures, it appears that for all the 
angles of attack, a separation region was formed behind the windward traffic barrier on the top surface of the upstream deck. Based on the mean pressure distribution, it seems that the reattachment length of the separation bubble, near the leading edge traffic barrier, increased with the angle of attack. For the positive wind angles of attack, it appears that the separated flow near the leading edge on the top surface did not reattach to the bridge surface as the pressures remained relatively large along this surface.

For the $-6^{\circ}$ wind angle of attack, it appears that flow separated just downstream of the leading edge on the bottom surface due to the observed large negative pressures over the windward inclined surface of the upstream deck. By increasing the wind angle of attack, positive pressures formed on this surface and increased in magnitude, implying that the separation region was moved downstream towards the windward transition point (see Fig. 5 (a)) on the bottom surface of the upstream deck.

On the downstream deck, for the $-6^{\circ}$ wind angle of attack, the top surface almost experienced positive pressures and it appears that the separated flow downstream to the leading edge on the bottom surface did not reattach through the entire surface, similar to the upstream girder. With the angle of attack increase, negative pressures reduced on the windward inclined surface of the downstream girder. By increasing the angle of attack, larger portion of the top surface experienced large negative pressures downstream to the windward traffic barrier, implying that separation grew in size with the angle increase. The large negative pressures observed near the leading edge of the windward corner of the downstream girder for the $-6^{\circ}$, moved towards the windward transition point on the bottom surface with the angle of attack increase corresponding to a move of separation 
region. As seen in Fig. 8, mean pressure distribution changed considerably with the angle of attack variation.

Forces and moments induced by the wind flow can be obtained by integration of the pressures over the cross sectional surface. For the $-6^{\circ}$ wind angle of attack, the drag force was created by the positive pressures on the top surfaces and negative pressures on the inclined surfaces. With the angle of attack increase, the positive pressure on the top surfaces reduced, till negative pressures were formed on the top surfaces for the positive wind angles of attack. Based on the pressure data, it seems that the drag force was reduced with the angle of attack increase from negative angles to zero angle, due to the reduction of the positive pressure on the top surface. By additional angle of attack increase, large negative pressure formed on the top surfaces, which seems to increase the drag. The lift force was mainly generated by the effective pressures on the top and bottom surfaces. With the angle of attack increase negative pressures on the bottom surface reduced and negative pressures formed and increased on the top surfaces, leading to the reduction of the expected negative lift in magnitude. Due to the significant modifications of the pressure patterns on the upstream girder, negative moments created by the negative lift on the upstream girder increased to positive values when the angle of attack increased.

From Figs. 8-9, it is noticed that the pressure distribution on the upstream deck was almost insensitive to the gap width. However, the pressure distribution on the downstream deck changed noticeably with the change of the gap width, particularly for the zero angle of attack. As the gap width increased, the downstream girder became 
more exposed to the oncoming flow and the negative mean pressures near the windward transition point of the downstream deck increased.

The comparison of the pressure distribution on the downstream girder between the different gap widths, suggested different flow excitation mechanisms for the larger gap width (i.e. $\mathrm{b} / \mathrm{C}=1.0$ ) compared to the smaller gap widths (i.e. $\mathrm{b} / \mathrm{C}=0.5$ and 0.75 ). With the gap width increase, negative pressures on the windward inclined surface decreased and positive pressures formed on this surface due to the direct exposure to the oncoming flow. For the largest gap width (i.e. $\mathrm{b} / \mathrm{C}=1.0$ ), large negative pressures were noticed downstream of the windward traffic barrier, implying that a separation region was formed in this region (see Fig. 8f) and that the downstream girder was immersed in the wake of the upstream girder. For the smaller gap widths (i.e. $\mathrm{b} / \mathrm{C}=0.5$ and 0.75 ), the large negative pressures observed near the windward leading edge of the inclined surface can be linked to flow separation in this region and possible ventilation of the flow from the top surface to the bottom surface through the gap.

Fluctuating pressure distributions on the upstream girder also confirmed the fact that flow around the upstream girder was almost independent of the gap width (see Fig. 9). The observed large fluctuating pressures on the windward side of the downstream girder were induced by the turbulent flow which was generated by the vortex shedding from the upstream girder. The wake of the upstream deck affected the separated shear layer on the downstream deck and increased the turbulence and fluctuations in pressures compared to the upstream deck. 
Upwind

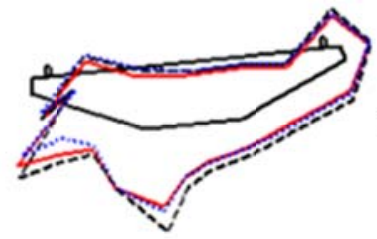

Downwind

(a) Upstream girder at $-6^{\circ}$

Upwind

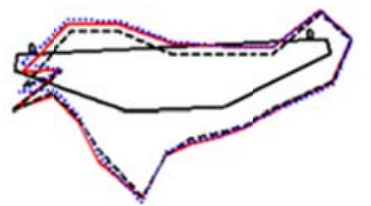

Downwind

(c) Upstream girder at $-3^{\circ}$

Upwind

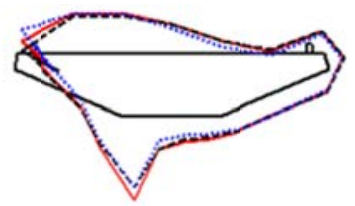

Downwind

(e) Upstream girder at $0^{\circ}$

Upwind

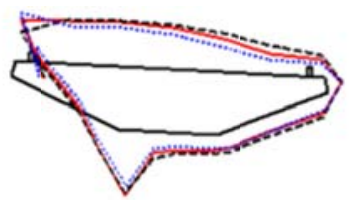

Downwind

(g) Upstream girder at $+3^{\circ}$

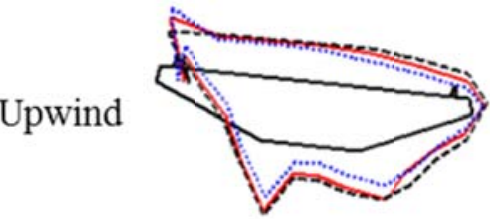

Downwind

(i) Upstream girder at $+6^{\circ}$
Upwind

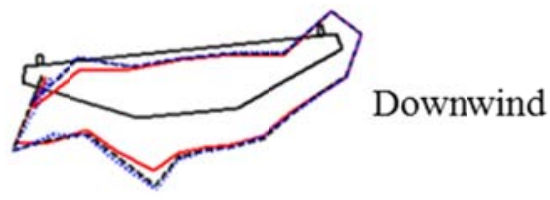

(b) Downstream girder at $-6^{\circ}$

Upwind

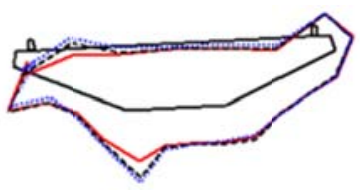

Downwind

Upwind

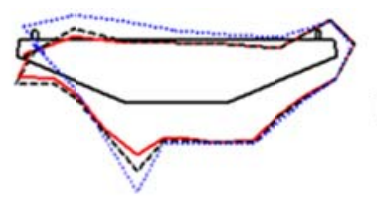

Downwind

(f) Downstream girder at $0^{\circ}$

Upwind

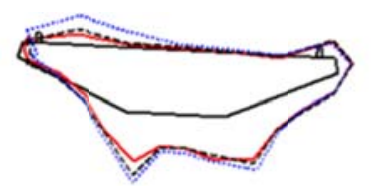

Downwind

Figure 8: The effect of gap width on the mean pressure distribution

$$
\left(C_{\bar{P}}=1.0, \longmapsto\right), R e=1.4 \times 10^{5},-b / C=0.5, \cdots b / C=0.75, \cdots \cdots \cdots \cdots \cdot b=1.0
$$


Upwind

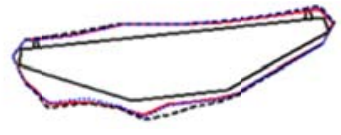

Downwind

(a) Upstream girder at $-6^{\circ}$

Upwind

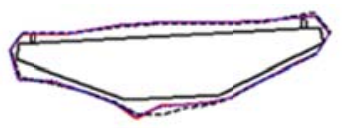

Downwind

(c) Upstream girder at $-3^{\circ}$

Upwind

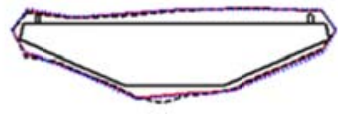

Downwind

(e) Upstream girder at $0^{\circ}$

Upwind

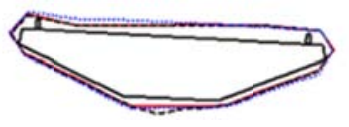

Downwind

(g) Upstream girder at $+3^{\circ}$

Upwind

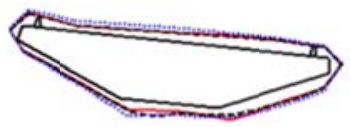

Downwind

(i) Upstream girder at $+6^{\circ}$
Upwind

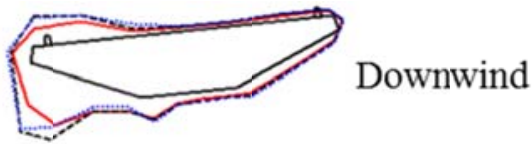

(b) Downstream girder at $-6^{\circ}$

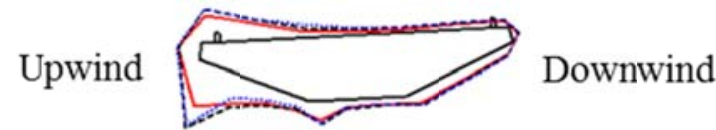

(d) Downstream girder at $-3^{\circ}$

Upwind

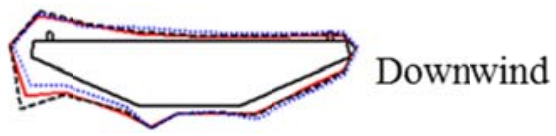

(f) Downstream girder at $0^{\circ}$

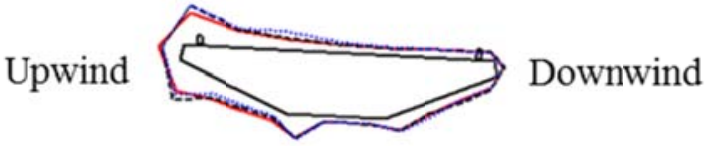

(h) Downstream girder at $+3^{\circ}$

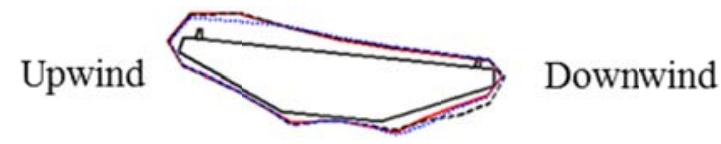

(j) Downstream girder at $+6^{\circ}$

Figure 9: The effect of gap width on the fluctuating pressure distribution $\left(C_{\sigma_{P}}=1.0, \longmapsto\right), R e=1.4 \times 10^{5},-b / C=0.5, \cdots b / C=0.75, \cdots \cdots \cdots \cdot b / C=1.0$

The results show that for the negative angles of attack, gap width played an important role on the vortices impinging on the downstream deck; as with the gap width increase, larger fluctuating pressures were noticed on the windward side of the downstream deck. For the zero wind angle of attack, an increase was observed in the fluctuating pressures with the gap width increase from $b / C=0.5$ to $b / C=0.75$. However, with the additional gap width increase, the downstream deck was less 
influenced by the impinging turbulent flow. This means that the downstream deck of the bridge model with $b / C=0.75$, experienced the largest effect from the turbulence generated by the vortex shedding from the upstream deck. For the positive wind angles of attack, almost no dependency on the gap width was observed for the fluctuating pressures.

\subsubsection{Effects of deck appurtenances}

\subsection{Effects of traffic barriers}

The effects of the traffic barriers on the mean and fluctuating pressure distributions, over the strip D of the 1:36 scaled model, are illustrated in Figs. 10-11. The observed differences in pressure distributions between the results from the 1:36 scaled model and the $1: 72$ scaled model with the $\mathrm{b} / \mathrm{C}=0.5$ gap width can be attributed to the $R e$ and turbulence effects which has been discussed in depth in a former study by the authors (Kargarmoakhar et al, 2015).

From Fig. 10, it can be noticed that the presence of the traffic barriers not only changed the pressure distribution over the top surfaces, but it also modified the pressure distribution over the bottom surfaces. The presence of traffic barriers caused a blockage effect with respect to the flow passing over the top surface of the deck. As a result, the flow was diverted towards the bottom surface which led to a wind speed increase over the bottom surface. From the Bernoulli equation, suctions increased due to the wind speed increase and as a result bottom surfaces experienced larger suctions compared with the bare section. Based on the mean pressure distributions observed, it is assumed that the bridge experienced larger negative lift for the condition equipped with the traffic barriers. 
For the bare deck, flow separation was highlighted by the large negative pressures on the top surface near the leading edge region. The small pressures observed downstream to these large negative pressures imply that the separated flow reattached at a short distance downstream. With the addition of the traffic barriers, the magnitude of the peak negative pressure on the windward side of the girder decreased and a separation region was highlighted behind the traffic barrier which tended to reattach in a longer length. Similar observations can be inferred from the fluctuating pressure distributions regarding the reattachment length on the top surface (see Fig. 11). It can be seen that for the bare condition, only a small region on the windward side of the top surface experienced highly turbulent flow. However, for the section equipped with barriers, a larger region experienced turbulent flow, implying a longer reattachment length.
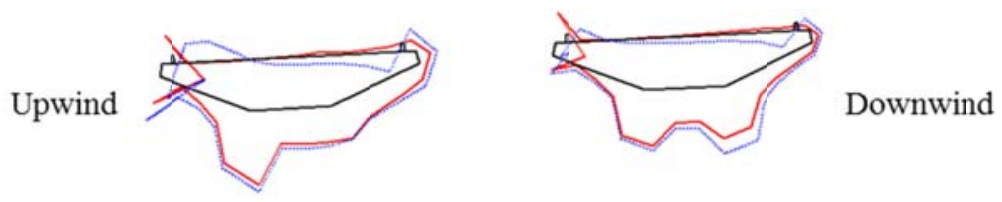

(a) Tilted at $-3^{\circ}, \operatorname{Re}=4 \times 10^{5}$
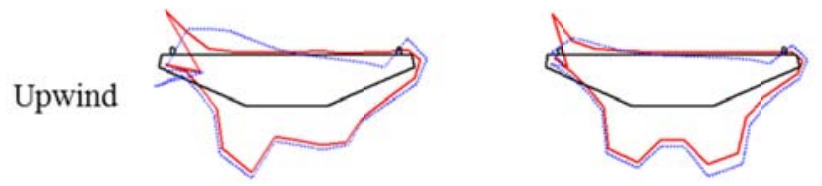

Downwind

(b) No Tilt (at $0^{\circ}$ ), $R e=4 \times 10^{5}$
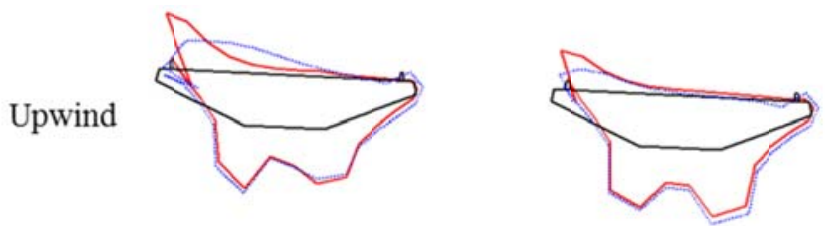

Downwind

(c) Tilted at $+3^{\circ}, R e=4 \times 10^{5}$

Figure 10: The effect of traffic barriers on the mean pressure distribution $\left(C_{\bar{P}}=1.0, \longmapsto\right), \longrightarrow$ Bare deck, $\cdots \cdots \cdots \cdots$ Bare deck + traffic barriers 

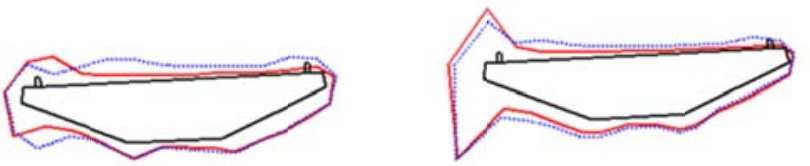

Downwind

(a) Tilted at $-3^{\circ}, R e=4 \times 10^{5}$

Upwind
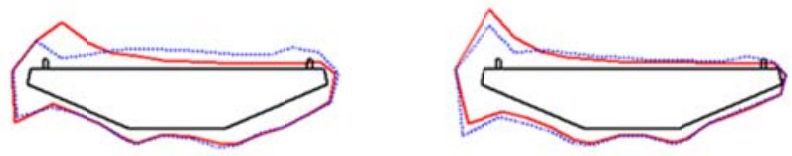

Downwind

(b) No Tilt (at $\left.0^{\circ}\right), R e=4 \times 10^{5}$

Upwind
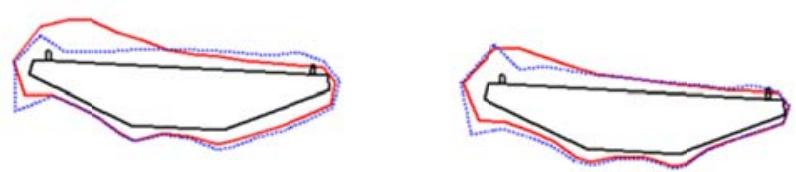

Downwind

(c) Tilted at $+3^{\circ}, R e=4 \times 10^{5}$

Figure 11: The effect of traffic barriers on the fluctuating pressure distribution

$\left(C_{\sigma_{P}}=1.0, \bullet\right),-$ Bare deck, $\cdots \cdots \cdots \cdots \cdot$ Bare deck + traffic barriers

\subsection{Effects of bike path on the upwind side and counterweight member on}

the downwind side

In order to evaluate the effects of the bike path and the counterweight member on the aerodynamic response of the bridge, the bike path was attached to the windward side of the upstream deck and the counterweight member was attached to the leeward side of the downstream deck. Due to construction and space limitations, pressure taps were only distributed across the bare cross section; therefore no pressure data was available around the bike path and the counterweight member. For this set of tests, the maximum testing wind speed was limited to $26.0 \mathrm{~m} / \mathrm{s}$, corresponding to $R e=2.6 \times 10^{5}$, based on the section depth. 
Figure 12 shows the comparison of the pressure distribution over the strip D for the bare deck section versus the section equipped with the bike path and the counterweight member. Based on the Fig. 12, the mean pressure distribution was barely affected by the addition of the bike path and the counterweight member. The fluctuating pressure distribution showed a little sensitivity to the bike path inclusion (only near the leading edge), i.e. including the bike path reduced the pressure fluctuations near the leading edge corresponding to a less turbulent flow near this area as the equipped section represented a more streamlined cross section.
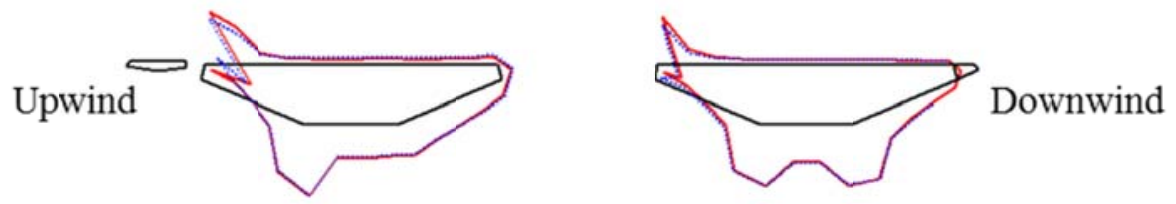

(a) Mean pressure distribution $\left(C_{\bar{P}}\right)$, No Tilt (at $\left.0^{\circ}\right), R e=2.6 \times 10^{5}$
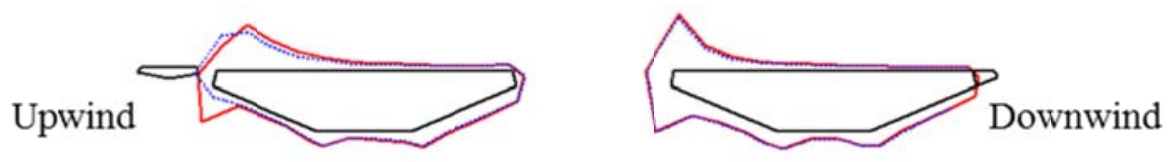

(b) Fluctuating pressure distribution $\left(C_{\sigma_{P}}\right)$, No Tilt (at $\left.0^{\circ}\right), R e=2.6 \times 10^{5}$

Figure 12: The effect of bike path on the mean pressure distribution $\left(C_{P}=1.0, \longmapsto\right),-$ Bare deck, $\cdots \cdots \cdots \cdots$ Bare deck + bike path + counterweight member

\subsubsection{Aerodynamic force coefficients}

Wind-induced loads acting on a bridge deck can be expressed in terms of two force components of Drag $\left(F_{D}\right)$ and Lift $\left(F_{L}\right)$ and pitching Moment $(M)$. As shown in Fig. 2, Drag is defined as the force component acting parallel to the wind direction, Lift is the force component perpendicular to the wind direction and pitching Moment is defined as the effective moment about the deck geometric center. Drag coefficient $\left(C_{D}\right)$, Lift 
coefficient $\left(C_{L}\right)$ and Moment coefficient $\left(C_{M}\right)$ are the normalized form of the aerodynamic forces and moment obtained using the dynamic pressure and model dimensions, as:

$$
C_{D}=\frac{F_{D}}{\frac{1}{2} \rho U^{2} L H}, C_{L}=\frac{F_{L}}{\frac{1}{2} \rho U^{2} L B}, C_{M}=\frac{M}{\frac{1}{2} \rho U^{2} L B^{2}}
$$

where, $F_{D}$ is the mean $\operatorname{drag}(\mathrm{N}), F_{L}$ is the mean lift $(\mathrm{N}), M$ is the mean pitching moment $(\mathrm{N} \cdot \mathrm{m}), \rho$ is the air density $\left(\mathrm{kg} / \mathrm{m}^{3}\right), U$ is the mean wind speed $(\mathrm{m} / \mathrm{s})$, and $B, H$ and $L$ represent the deck chord, deck height and the length of the model, respectively $(\mathrm{m})$. Mean wind generated loads are important in the design of long span bridges, e.g. smaller drag can reduce the construction costs significantly by requiring smaller and lighter sections for the cables, towers and the foundation. Fluctuating wind loads are also critical in the design since they concern the stability, lifetime serviceability and fatigue.

The bridge response under a turbulent wind can be estimated by predicting the wind-induced loads from quasi steady theory and combining the estimated loads with the equations of motion of the deck (Irwin, 1977; Larose, 2002). This method yields a set of formulas for the fluctuating wind loads in terms of the steady aerodynamic force coefficients and their derivatives with respect to the angle of attack. Equations (3) to (5) show the equations of motion for a bridge deck under a gusty wind, based on the quasi steady theory (Irwin, 1977):

$$
\begin{gathered}
M_{x} \ddot{x}+\left(G_{x}+\rho U B C_{D}\right) \dot{x}+K_{x} x+\left[1 / 2 \rho U B \frac{d C_{D}}{d \alpha} \dot{z}-1 / 2 \rho U^{2} B \frac{d C_{D}}{d \alpha} \theta\right]= \\
\frac{\rho U B}{2}\left[2 C_{D} u+\frac{d C_{D}}{d \alpha} w\right]
\end{gathered}
$$




$$
\begin{gathered}
M_{z} \ddot{z}+\left(G_{z}+1 / 2 \rho U B \frac{d C_{L}}{d \alpha}\right) \dot{z}+K_{z} z+ \\
{\left[\rho U B C_{L} \dot{x}-1 / 2 \rho U^{2} B \frac{d C_{L}}{d \alpha} \theta-1 / 2 \rho U n B \dot{\theta}\right]=\frac{\rho U B}{2}\left[2 C_{L} u+\frac{d C_{L}}{d \alpha} w\right]} \\
I_{\theta} \ddot{\theta}+\left(G_{\theta}+1 / 2 \rho B^{3} \frac{d C_{M}}{d \alpha} n U\right) \dot{\theta}+\left(K_{\theta}-1 / 2 \rho U^{2} B^{2} \frac{d C_{M}}{d \alpha}\right) \theta+\left[\rho U B^{2} C_{M} \dot{x}+\right. \\
\left.1 / 2 \rho U B^{2} \frac{d C_{M}}{d \alpha} \dot{z}\right]=\frac{\rho U B^{2}}{2}\left[2 C_{M} u+\frac{d C_{M}}{d \alpha} w\right]
\end{gathered}
$$

where, $x, z$ and $\theta$ are the bridge responses in the horizontal, vertical and torsional directions, single and double dotted parameters are the derivatives of the bridge responses with respect to the time, $M_{x}, M_{z}$ and $I_{\theta}$ are the effective inertias of the deck in horizontal, vertical and torsional motions, $G_{x}, G_{z}$ and $G_{\theta}$ are the effective viscous damping in the three directions, $K_{x}, K_{z}$ and $K_{\theta}$ are the effective stiffness in the three directions.

Based on the quasi steady theory, the steady force coefficients can be important on the aerodynamic damping, aerodynamic stiffness and buffeting forces (Larose, 2002). Keeping in mind that the aerodynamic stiffness is often negligible compared to the structural stiffness and the fact that coupling terms are often small, the aerodynamic damping remains as the most important wind induced effect (Larose, 2002). For bridges at low wind angles of attack, $C_{D}$ is often a positive value that will lead to a positive aerodynamic damping in the horizontal direction (see Eq. (3)). However, based on the Eq. (4), the vertical aerodynamic damping is a factor of $d C_{L} / d \alpha$ which can reduce the total vertical damping when it is negative. As a result, there is a potential for galloping at a certain wind speed when the section experiences negative $d C_{L} / d \alpha$. Eqs. (3)-(5) show that the fluctuating loads due to the turbulence in the approaching wind (buffeting loads) 
are also dependent to the steady force coefficients and their derivatives with respect to the angle of attack.

The quasi steady assumption does not consider the effect of the out of phase components of the aerodynamic forces that can also contribute to the aerodynamic damping and stiffness of the bridge. The out of phase components result from the structural motion and can be obtained from testing a sectional model in a dynamic test rig with at least two degrees of freedom in the heaving and torsional directions.

The quasi steady theory equations are presented in this section to show the importance of the steady forces and moment coefficients and their derivatives with respect to the angle of attack on the aerodynamic response of a bridge deck. In the following parts, the effects of deck details are evaluated on the steady forces and moment coefficients to highlight how changing the geometry details can change the aerodynamic response of the studied cross section.

\subsubsection{Effects of gap width}

Forces and moments were obtained both by direct measurement from the load cells and by the integration of the surface pressures. In order to reduce the randomness error in the estimation of force and moment coefficients from the pressure measurement, the average of the measured pressures over the four strips was reported (Fig. 4 shows the distribution of the pressure tapped strips). It was assumed that the average of the measured forces and moment over the strips A, B and D were effective for $2 / 3$ of the model length and measured forces and moment over the strip $C$ were effective for $1 / 3$ of the model length. 
Figure 13 shows the force and moment coefficients for the model with the largest gap width $(\mathrm{b} / \mathrm{C}=1.0)$ at different wind angles of attack. It can be observed that the lift coefficient obtained from the pressure integration matched very well with the results from the direct force measurement by the load cells. The discrepancy observed in the drag coefficient between the two methods of measurement can be attributed to the fact that the traffic barriers were not equipped with the pressure taps. For the positive wind angles of attack, traffic barriers were sheltered by the deck and the results from the pressure measurement and the load cells matched each other well. However, for the negative and zero wind angles of attack the results from the two measurement methods did not agree, showing the direct contribution of the traffic barriers on the drag. For the non-positive wind angles of attack, moment coefficient resulted by the pressure measurement was slightly different from the measured moment coefficient by the load cells. This can be explained similar to what was mentioned for the drag coefficient, as for both cases the difference was due to the fact that the barriers were not equipped to pressure taps.

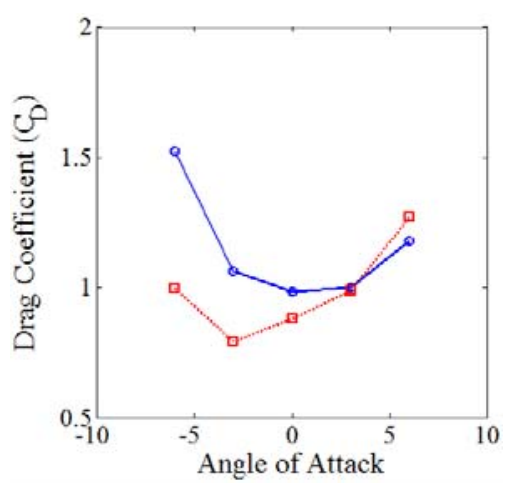

(a) Drag coefficient

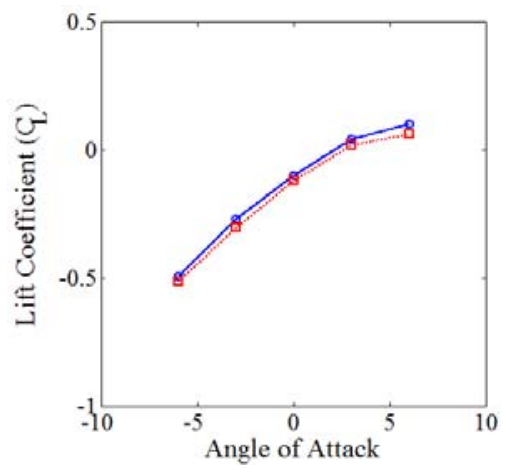

(b) Lift coefficient

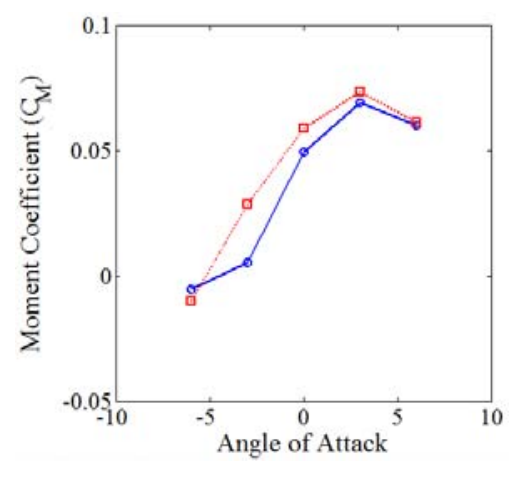

(c) Moment coefficient

Figure 13: Force and moment coefficients for the model with the largest gap width (b/C=1.00), $R e=1.4 \times 10^{5}, \rightarrow-$ Load cells, $\cdots \boxminus \cdots$ Pressure measurement 
Figure 14 shows the effect of gap width on the force and moment coefficients measured with the load cells. For the zero wind angle of attack which is the most important wind flow direction, the drag coefficient was almost insensitive to the gap width increase. As shown in Fig. 8(f), by increasing the gap width from $b / C=0.75$ to 1.0 , the pressure distribution was modified around the windward side of the downstream girder. However, the overall drag remained constant and as a result the drag coefficient did not change with the gap width increase. For the positive wind angles of attack, increasing the gap width led to a slight increase in the drag coefficient while for negative wind angles of attack no clear trend was observed. The results for the positive wind angles of attack confirms the results from Kwok et al (2012) in which it was observed that drag coefficient increased with the gap width increase. As mentioned previously, larger drag corresponds to higher construction costs by requiring larger sections for cables, towers and the foundation.

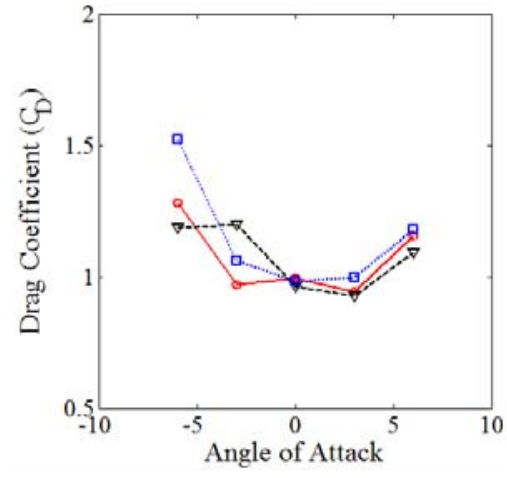

(a) Drag coefficient

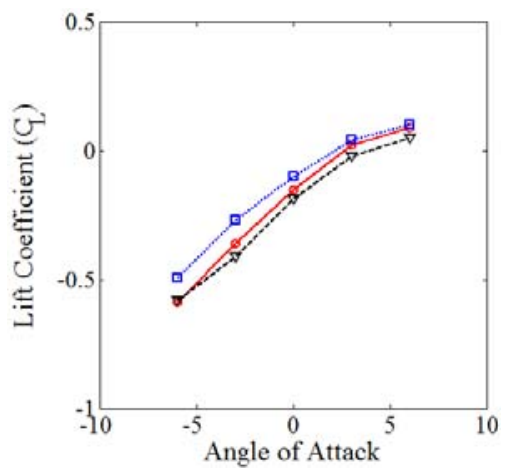

(b) Lift coefficient

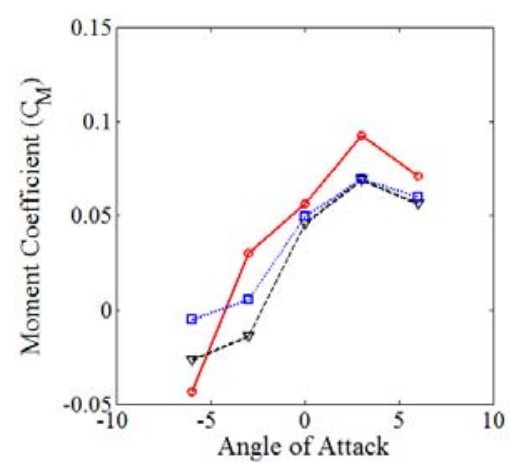

(c) Moment coefficient

Figure 14: The effect of gap width on the force and moment coefficients

$$
R e=1.4 \times 10^{5}, \rightarrow \mathrm{b} / \mathrm{C}=0.5,--\nabla-\mathrm{b} / \mathrm{C}=0.75, \cdots \mathrm{G} \cdots \mathrm{b} / \mathrm{C}=1.00
$$

Figure $14 \mathrm{~b}$ shows that the lift coefficient remained relatively constant with the gap width increase from $b / C=0.5$ to $b / C=0.75$ and decreased in magnitude with the 
additional gap width increase to $b / C=1.0$, for almost all the different wind angles of attack tested. It was also observed that the trend of the lift coefficient variation with respect to the angle of attack did not change for the different gap widths, which is in contrast to the observations of Kwok et al (2012). Kwok et al (2012) noted that the slope of the lift coefficient variation with respect to the angle of attack had a decreasing trend with the gap width increase. Smaller lift coefficient slope corresponds to smaller aerodynamic damping in the vertical degree of freedom, as mentioned earlier.

Figure 15 shows the lift coefficient calculated by normalizing the lift force with the single chord length (C) instead of the variable total width (B), shown by $C_{L, C}$. It was observed that increasing the gap width from $b / C=0.5$ to $b / C=0.75$ resulted in larger lift coefficients $\left(C_{L, C}\right)$ while additional increase to $b / C=1.0$ reversed this trend and created smaller lift coefficients. Figure 14(c) shows that the moment coefficient decreased with the gap width increase for all the different wind angles of attack.

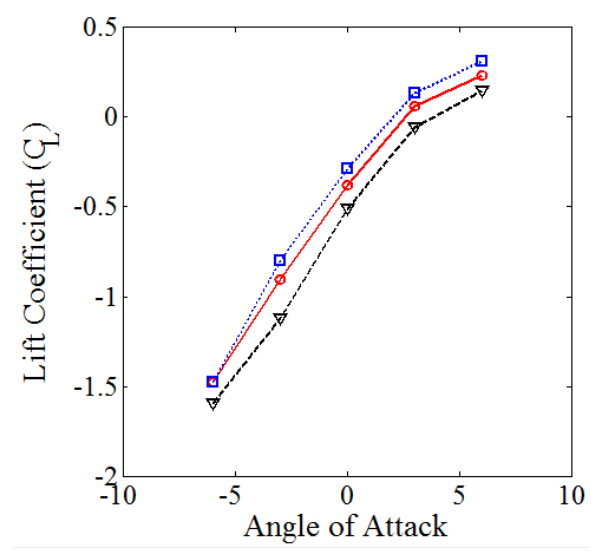

Figure 15: Lift coefficient by load cells normalized to $\mathrm{C}\left(C_{L, C}\right)$

$$
R e=1.4 \times 10^{5}, \rightarrow \mathrm{b} / \mathrm{C}=0.5,-\nabla-\mathrm{b} / \mathrm{C}=0.75, \cdots \boxminus \mathrm{b} / \mathrm{b} / \mathrm{C}=1.00
$$

The effect of the gap width on the derivative of the force and moment coefficients with respect to the angle of attack (force and moment coefficients slopes) for the zero 
wind angle of attack is shown in Fig. 16. It can be observed that $d C_{D} / d \alpha$ was close to zero for the largest $(b / C=1.0)$ and the smallest $(b / C=0.5)$ gap widths. However, for the model with the $\mathrm{b} / \mathrm{C}=0.75$ gap width, negative $d C_{D} / d \alpha$ was noticed. The value of the $d C_{D} / d \alpha$ is important on the aerodynamic coupling and buffeting forces for the horizontal direction as shown in Eq. (3). Negative $d C_{D} / d \alpha$ corresponds to the reduction of buffeting forces in the along wind direction. According to the Fig. 16, with the gap width increase $d C_{L} / d \alpha$ was decreased slightly with no change in sign. $d C_{L} / d \alpha$ plays an important role in the aerodynamic damping and buffeting forces in the vertical direction. Positive $d C_{L} / d \alpha$ attributes to positive aerodynamic damping and at the same time larger buffeting forces. The moment coefficient derivative with respect to the angle of attack changed negligibly with the gap width variation. $d C_{M} / d \alpha$ has contribution to the aerodynamic coupling, aerodynamic damping, aerodynamic stiffness and also buffeting forces in the torsional direction.

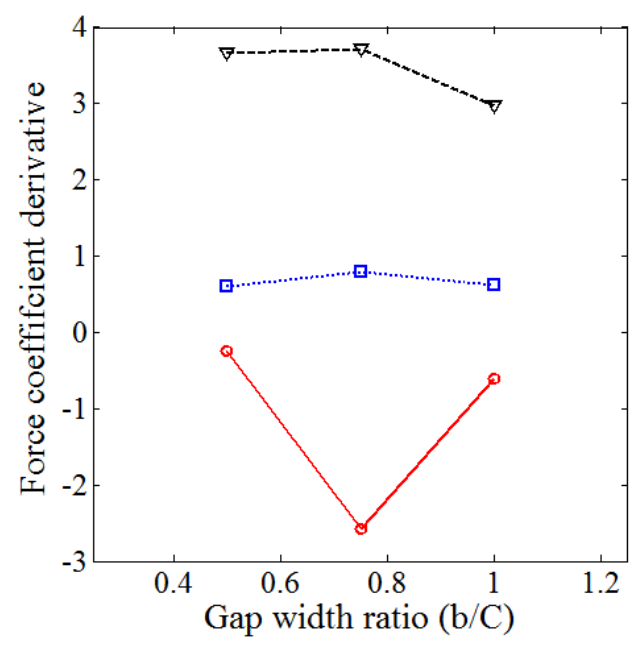

Figure 16: Derivative of force and moment coefficients with respect to the angle of attack for the zero wind angle of attack

$$
R e=1.4 \times 10^{5}, \multimap d C_{D} / d \alpha,--\nabla-d C_{L} / d \alpha, \cdots \boxminus \cdots d C_{M} / d \alpha
$$




\subsubsection{Effects of deck appurtenances}

\subsection{Effects of traffic barriers}

Figure 17 shows the force and moment coefficients measured by the load cells and the pressure taps for the bare deck section and the section equipped with the traffic barriers, using the 1:36 scaled model. The aerodynamic coefficients calculated from the pressure measurement were averaged over the 6 pressure tapped strips, similar to the method applied in the previous section to study the effect of gap width. For the bare deck section, the forces and moment coefficients obtained from the pressure measurement were consistent with the forces and moment coefficients measured with the load cells within a reasonable range. It can be seen that adding the traffic barriers to the bare deck cross section resulted in a higher drag coefficient for all the different angles of attack, particularly for the negative wind angles of attack in which the traffic barriers were not sheltered by the deck. To obtain the direct contribution of the traffic barriers on the drag coefficient for the equipped deck, the results from the pressure measurement were compared to the load cells data. It can be inferred that the drag coefficient variations were mostly due to the direct effect of the wind loading on the traffic barriers.

The comparison of the lift coefficient for the bare deck with the equipped deck shows that adding the traffic barriers resulted in larger negative lift coefficients. This can be explained by the blockage effect of the traffic barriers on the top surface. As a result, the flow was deviated towards the bottom surface and the wind speed increased over the bottom surface, creating larger suctions on the bottom surface. The presence of the traffic berries also modified the pressure distribution over the top surface by reducing the peak negative pressure and increasing the reattachment length. The flow modification over the 
top and bottom surfaces due to the addition of traffic barriers generated larger negative lift coefficient. The moment coefficient showed less sensitivity to the presence of traffic barriers compared to the forces coefficients and was mainly a function of wind angle of attack. Similar to the lift coefficient, the moment coefficient showed a positive slope as a function of wind angle of attack.

Table 1 shows the effect of the traffic barriers on the derivative of the force and moment coefficients with respect to the angle of attack (force and moment coefficients slopes), for zero angle of attack. It can be noticed that the derivative of drag coefficient had higher sensitivity to the inclusion of traffic barriers. The derivative of moment coefficient had a similar pattern to the derivative of the lift coefficient. For both lift and moment, the derivative had a positive value that slightly increased with the addition of the traffic barriers.

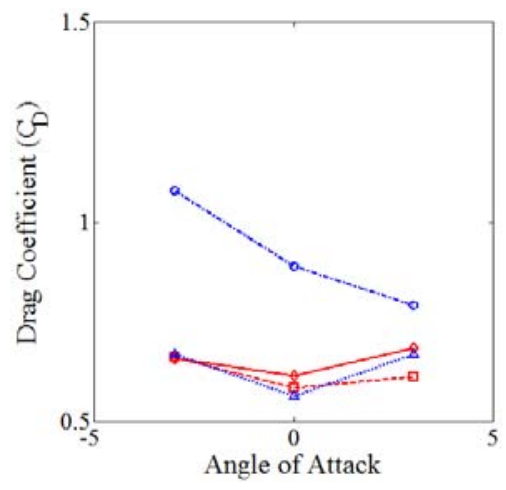

(a) Drag coefficient

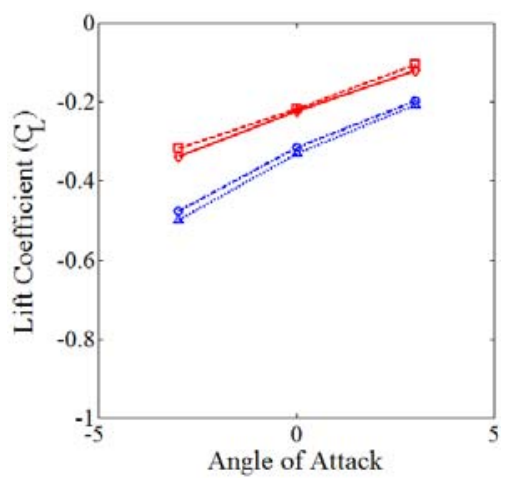

(b) Lift coefficient

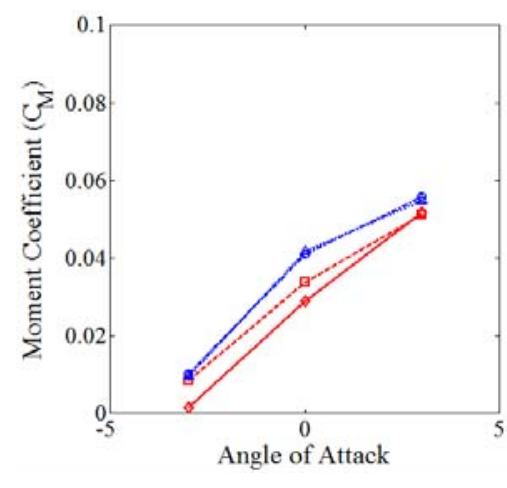

(c) Moment coefficient

Figure 17:The effect of traffic barriers on the force and moment coefficients

$$
R e=4.0 \times 10^{5}
$$

Bare deck:-- -- by load cell, $\rightarrow$ by pressure measurement

Bare deck + Traffic barriers: $\cdots \cdots$ by load cell, $\cdots \cdots \cdots$ by pressure measurement 
Table 1: Derivative of force and moment coefficients with respect to the angle of attack for the zero wind angle of attack, $R e=4.0 \times 10^{5}$

\begin{tabular}{|c|c|c|c|}
\hline & $d C_{D} / d \alpha$ & $d C_{L} / d \alpha$ & $d C_{M} / d \alpha$ \\
\hline Bare deck & -0.46 & 2.05 & 0.41 \\
\hline Bare deck + traffic barriers & -2.73 & 2.68 & 0.44 \\
\hline
\end{tabular}

\subsection{Effects of bike path on the windward side and counterweight member on}

the leeward side

Table 2 shows the effect of bike path and counterweight member on the force and moment coefficients for the section at zero wind angle of attack and $R e=2.6 \times 10^{5}$. In order to derive the lift and moment coefficients, the total width of the section equipped with the bike path and counterweight member was considered to be equal to $2.18 \mathrm{~m}$ which was $12 \%$ wider than the bare section with $1.94 \mathrm{~m}$ width. It can be noticed that only the drag coefficient changed with the inclusion of the bike path leading to a $5 \%$ reduction. The reduction in the drag coefficient can be attributed to the more streamlined cross section of the deck equipped with the bike path. Lift and moment coefficients were almost insensitive to the inclusion of the bike path and counterweight member when considering the increased width for non-dimensionalization.

Table 2: Force and moment coefficients for the zero wind angle of attack from load cells, $R e=2.6 \times 10^{5}$

\begin{tabular}{|c|c|c|c|}
\hline & $C_{D}$ & $C_{L}$ & $C_{M}$ \\
\hline Bare deck & 0.61 & -0.16 & 0.04 \\
\hline Bare deck + bike path + counterweight member & 0.58 & -0.16 & 0.04 \\
\hline
\end{tabular}

\subsubsection{Vortex shedding}

Flow passing across a sharp edged bluff section usually separates at the edge points, leading to the formation of vortices around the section. Vortices shedding from the bridge section contribute to the formation of fluctuating pressures around the section. 
As a result, the section experiences cross wind forces that fluctuate periodically. When the frequency of the vortex shedding approaches one of the structural oscillation frequencies, a resonant type response called vortex induced vibration (VIV) happens. VIV creates large amplitude but self-limiting motions. Strouhal number $S t$ is a nondimensional parameter that defines the dominant frequency of the fluctuations in the cross-wind force and is expressed by the following equation:

$$
S t=\frac{f H}{U}
$$

where, $f$ is the frequency of vortex shedding $(\mathrm{Hz})$. St is a function of structure's geometry, turbulence intensity and $R e$. In this study, the vortex shedding frequency was obtained from the power spectral density of the fluctuating lift force on the section. The frequency corresponding to the peak evident in the fluctuating lift spectrum shows the frequency of the vortex shedding (Kwok et al, 2012; Schewe and Larsen, 1998). Three mechanisms have been distinguished for the vortex shedding from a twin girder bridge (Kwok et al, 2012). The first and second mechanisms have been attributed to the vortex shedding from the trailing edge of the upstream and downstream decks. The third mechanism has been attributed to the impingement of the vortices in the wake of the upstream deck onto the windward side of the downstream deck. The results presented in the following sections are based on the measured pressure data over the strip B location for the effect of gap width and the measured pressure data over the strip D for the effect of deck appurtenances. 


\subsubsection{Effects of gap width}

The effect of gap width on the vortex shedding frequency was illustrated by plotting the lift coefficient spectra multiplied by the frequency against reduced frequency $(f H / U)$ for different test configurations at zero wind angle of attack, as shown in Fig. 18. Spectral peaks were noticed for each of the test configurations, indicating that all the configurations were prone to vortex shedding. It can be observed that widening the gap width was accompanied by an increase in the vortex shedding frequency. By increasing $b / C$ from 0.75 to 1.0 , the energy level was decreased implying that the fluctuating lift coefficient was reduced with this gap increase. This can be explained from the distribution of the fluctuating pressure around the downstream girder as shown in Fig. 9(f). From Fig. 9(f), it can be seen that the downstream girder was less influenced by the vortex shedding from the upstream girder and, as a result, the fluctuating lift coefficient was reduced.

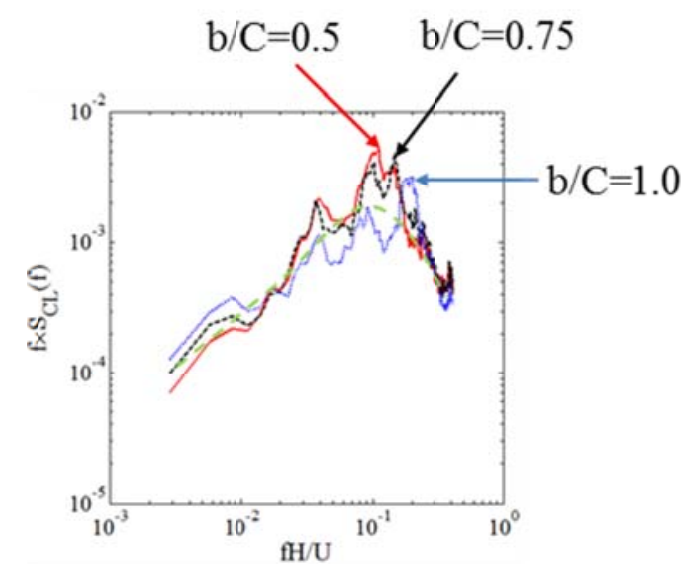

Figure 18: Lift coefficient spectra for the zero wind angle of attack $R e=1.4 \times 10^{5}$

$-b / C=0.5, \cdots b / C=0.75, \cdots \cdots \cdots \cdot b=1.0,---$ approximated buffeting spectra 
Figure 19 shows the variation of the Strouhal number with the gap to single deck width ratio $(b / C)$ for different angles of attack. It can be seen that the vortex shedding frequency was sensitive to the gap width for the cases of $0^{\circ}$ and $+3^{\circ}$ wind angles of attack, showing an increasing trend with the gap width increase. The increase of the Strouhal number is of importance as the wind velocity at which the resonant motion begins decreases with the Strouhal number increase. As a result, the risk of bridge experiencing vortex induced vibration is higher when the corresponding Strouhal number is larger.

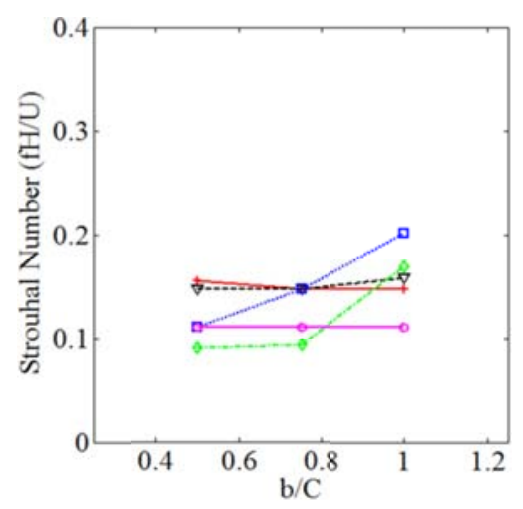

Figure 19: Strouhal Number as a function of gap to single deck width ratio $(\mathrm{b} / \mathrm{C})$ $R e=1.4 \times 10^{5}$, Angle of attack: $+-6^{\circ}, \cdots-3^{\circ}, \cdots \boxminus 0^{\circ}, \cdots+3^{\circ},-\circ+6^{\circ}$

The comparison of the effect of the deck configuration on the lift coefficient spectra for each individual deck is given in Fig. 20. This is mostly useful for the case that the two decks have independent structural systems, such as building a new bridge besides an older bridge. It can be seen that the peak in the spectra was more pronounce on the downstream girder. As a result, it can be concluded that the dominant mechanism in this case is due to the impingement of the vortices from the upstream girder onto the downstream girder. Among all the three different gap width configurations, $b / C=0.75$ 
was the most critical one as it showed larger peaks at higher levels of energy, corresponding to larger fluctuating lift forces. Figure 21 shows that the vortex shedding occurs at a higher frequency around the downstream deck, compared to the upstream deck. It is also evident that the overall fluctuating lift is larger on the downstream deck, due to the larger area under the lift coefficient spectra.

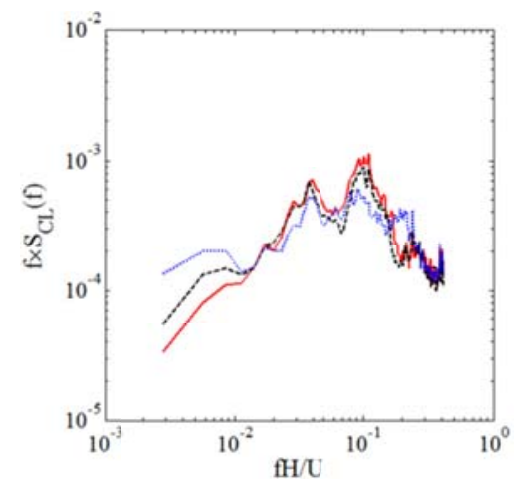

(a) Upstream deck

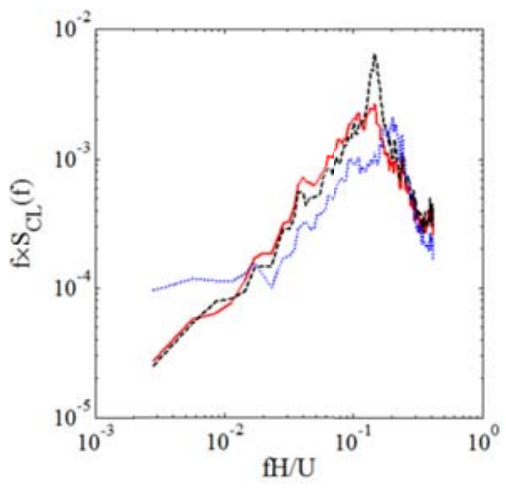

(b) Downstream deck

Figure 20: Lift coefficient spectra for the zero wind angle of attack $R e=1.4 \times 10^{5}$ $-b / C=0.5, \cdots-\cdots / C=0.75, \cdots \cdots \cdots \cdot b / C=1.0$

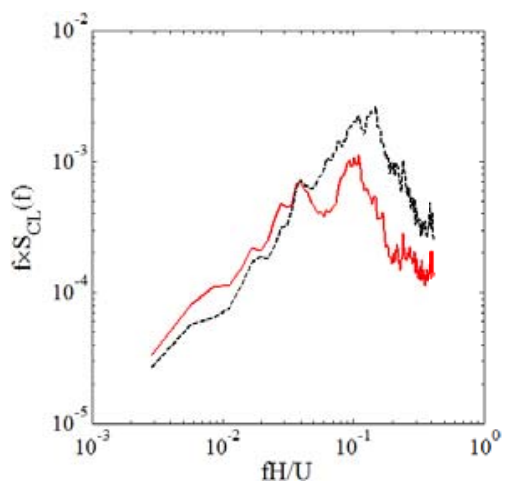

(a) $b / C=0.5$

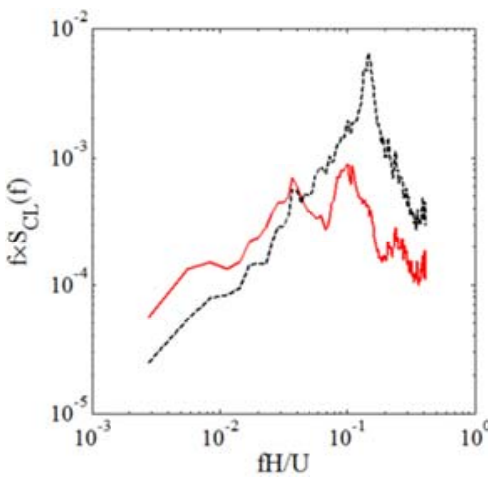

(b) $b / C=0.75$

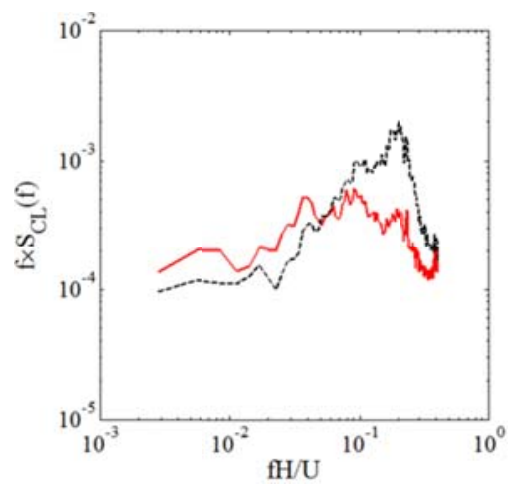

(c) $\mathrm{b} / \mathrm{C}=1.0$

Figure 21: Lift coefficient spectra for the zero wind angle of attack $R e=1.4 \times 10^{5}$

— Upstream deck, ---.- Downstream deck 


\subsubsection{Effects of deck appurtenances}

\subsection{Effects of traffic barriers}

Figure 22 shows the effect of traffic barriers on the lift coefficient spectra for the zero wind angle of attack. It can be seen that including the traffic barriers on the top surface of the bare deck, resulted in the formation of a new peak in the lift coefficient spectra. Thus, traffic barriers strengthened vortex shedding around the bridge.

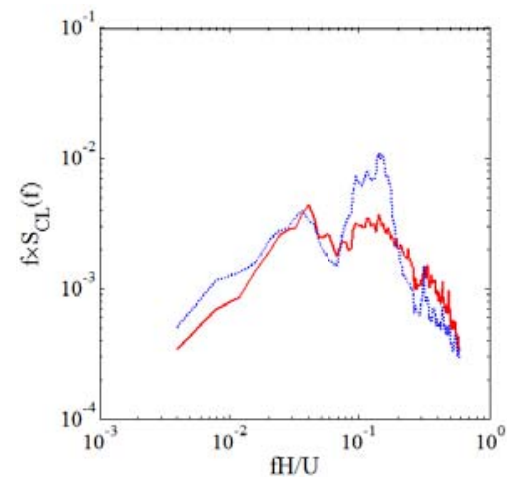

Figure 22: Effect of traffic barriers on the lift coefficient spectra $R e=4.0 \times 10^{5}$

— Bare deck, .......... Bare deck + traffic barriers

\subsection{Effects of bike path on the windward side and counterweight member on} the leeward side

A comparison of the lift coefficient spectra between the bare deck and the bare deck equipped with the bike path and counterweight member is shown in Fig. 23, for zero angle of attack. Based on Fig. 23, the high frequency part of the spectra matched very well between the two conditions. However, a difference is noticed between the low frequency part of the two conditions. It can be concluded that the addition of the bike path reduced the wind excitation at low frequencies but barely changed the wind excitation at high frequencies. 


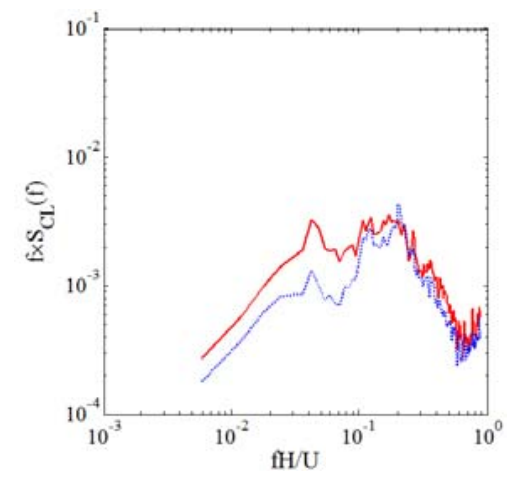

Figure 23: Effect of bike path on the normalized lift coefficient spectra $R e=2.6 \times 10^{5}$

— Bare deck, ......... Bare deck + bike path + counterweight member

\subsection{Summary and Conclusion}

The effects of the deck details, including the gap width, traffic barriers and the bike path were evaluated on the aerodynamic performance of a twin deck bridge through experiments. Pressures were measured along the chord length of the models to capture the effect of the deck details on the pressure distribution. Wind generated loads were also measured by load cells to capture the global effects of the deck details on the aerodynamic performance of the bridge. Three different gap to single deck width ratios of $0.5,0.75$ and 1.0 were considered to evaluate the effect of gap width. The effect of the deck appurtenances including the traffic barriers and an attached bike path was also studied by including these features in the model with the smallest gap width.

From the tests on the gap width, the following conclusions can be drawn:

1. Changing the gap width had a significant effect on the pressure distribution around the downstream deck, particularly for the smaller wind angles of attack i.e. $-3^{\circ}, 0^{\circ}$ 
and $+3^{\circ}$. However, the pressure distribution around the upstream deck was almost insensitive to the gap width change.

2. From the mean pressure distribution around the model with the smallest gap width at zero wind angle of attack condition, it can be hypothesized that the wind flow was ventilated through the gap. This created negative pressures near the leading edge of the downstream deck. With the increase of the gap ratio from 0.75 to 1.0 , positive pressures formed on the windward inclined surface of the downstream girder similar to the pressure distribution on the windward side of the upstream girder. It is hypothesized that for the largest gap ratio, the downstream girder was more exposed to the oncoming flow and was less sheltered by the upstream girder.

3. The force and moment coefficients showed sensitivity to the gap width increase by showing a slight increase in the drag and a decrease in the lift. The pattern of the variation of each of the force and moment coefficients with respect to the angle of attack was almost similar for all the different gap width conditions studied. The maximum changes observed in the mean drag coefficient between the different gap widths was equal to $28 \%, 24 \%, 3 \%, 7 \%$ and $8 \%$ for the $-6^{\circ},-3^{\circ}, 0^{\circ},+3^{\circ}$ and $+6^{\circ}$ wind angles of attack, respectively. The $d C_{L} / d \alpha$ decreased around $25 \%$ when the gap to single deck width ratio increased from 0.75 to 1.0 for the zero wind angle of attack condition. $d C_{L} / d \alpha$ is important for determination of vertical buffeting loads.

4. Spectral peaks were noticed in the lift coefficient spectra of the studied cases, signifying the presence of vortex shedding. For zero and $+3^{\circ}$ wind angles of attack, an increasing trend was observed for the Strouhal number with the gap width increase. Strouhal number increased by about $80 \%$ with the gap width increase for 
zero and $+3^{\circ}$ angles of attack, meaning that VIV can happen for the largest gap width at wind speeds $45 \%$ smaller than the corresponding wind speeds for the smallest gap width.

5. Vortex shedding was more pronounced around the downstream deck and at larger frequencies compared to the upstream deck.

Results from studying the effect of deck appurtenances can be summarized as follows:

1. The mean pressure distribution was significantly influenced by the inclusion of the traffic barriers. The traffic barriers changed not only the local pressures on the top surface, but also they increased the negative pressures over the bottom surface, due to the blockage effect on the top surface.

2. The pressure modifications due to the traffic barriers and the direct effect of the wind on the barriers resulted in the formation of a larger lift and drag. With the inclusion of the traffic barriers, the drag coefficient increased around $64 \%, 52 \%$ and $30 \%$ for the $-3^{\circ}, 0^{\circ}$ and $+3^{\circ}$ wind angles of attack, respectively. Also, due to the inclusion of the traffic barriers, the lift coefficient increased around $63 \%, 75 \%$ and $110 \%$ for the $-3^{\circ}$, $0^{\circ}$ and $+3^{\circ}$ wind angles of attack, respectively. While these percent changes in lift seem large it should be noted that the overall magnitude of $C_{L}$ at zero angle of attack is small. Therefore the changes are probably not significant from a practical point of view. Regarding the derivative of the aerodynamic coefficients with respect to the angle of attack, only the derivative of the drag coefficient showed sensitivity to the inclusion of the traffic barriers. 
3. The mean pressure distribution and the measured loads showed little sensitivity to the inclusion of the bike path and counterweight member in the simulation of the cross sectional shape.

4. It was observed that adding the traffic barriers enhanced the vortex shedding around the section. The bike path had much less effect.

\subsection{Acknowledgments}

This research was supported by the National Science Foundation (NSF) [NSF MRI Award CMMI-0923365] and Florida Center of Excellence in Hurricane Damage Mitigation and Product Development for acquisition of instruments. The authors also would like to acknowledge Maryam Asghari Mooneghi, Walter Conklin and Roy Liu Marques of Wall of Wind, FIU for their unreserved input during testing. The findings reported in this article are those of the authors alone, and do not necessarily represent the views of sponsoring agencies. We also acknowledge the contribution of T.Y. Lin International in providing us with information on the East Bay Bridge. The results provided in the paper do not extrapolate directly to the East Bay Bridge as the geometry was simplified for the purposes of the present research.

\subsection{References}

Bruno, L., Mancini, G., 2002. Importance of Deck Details in Bridge Aerodynamics. Structural Engineering International 12, 289-294.

Fransos, D., Bruno, L., 2010. Edge degree-of-sharpness and free-stream turbulence scale effects on the aerodynamics of a bridge deck. Journal of Wind Engineering and Industrial Aerodynamics 98, 661-671.

Gu, M., Zhang, R., Xiang, H., 2001. Parametric study on flutter derivatives of bridge decks. Engineering Structures 23, 1607-1613. 
Irwin, P., Cooper, K., Girard, R., 1979. Correction of distortion effects caused by tubing systems in measurements of fluctuating pressures. Journal of Wind Engineering and Industrial Aerodynamics 5, 93-107.

Irwin, P.A., 1977. Wind Tunnel and Analytical Investigations of the Response of Lions Gate Bridge To Turbulent Wind. National Research Council of Canada, National Research Council of Canada, p. 62.

Jones, N.P., Scanlan, R.H., Sarkar, P.P., Singha, L., 1995. The effect of section model details on aeroelastic parameters. Journal of Wind Engineering and Industrial Aerodynamics 54-55, 45-53.

Kargarmoakhar, R., Chowdhury, A.G., Irwin, P.A., 2015. Reynolds Number Effects on Twin Box Girder Long Span Bridge Aerodynamics. Wind and Structures.

Kwok, K.C.S., Qin, X.R., Fok, C.H., Hitchcock, P.A., 2012. Wind-induced pressures around a sectional twin-deck bridge model: Effects of gap-width on the aerodynamic forces and vortex shedding mechanisms. Journal of Wind Engineering and Industrial Aerodynamics 110, 50-61.

Laima, S., Li, H., Chen, W., Li, F., 2013. Investigation and control of vortex-induced vibration of twin box girders. Journal of Fluids and Structures 39, 205-221.

Larose, G.L., 2002. The dynamic action of gusty winds on long-span bridges, Department of Structural Engineering and Materials. Technical University of Denmark, p. 179.

Larsen, A., Savage, M., Lafrenière, A., Hui, M.C.H., Larsen, S.V., 2008. Investigation of vortex response of a twin box bridge section at high and low Reynolds numbers. Journal of Wind Engineering and Industrial Aerodynamics 96, 934-944.

Li, H., Laima, S., Ou, J., Zhao, X., Zhou, W., Yu, Y., Li, N., Liu, Z., 2011. Investigation of vortex-induced vibration of a suspension bridge with two separated steel box girders based on field measurements. Engineering Structures 33, 1894-1907.

Li, Q.S., Melbourne, W.H., 1995. An experimental investigation of the effects of freestream turbulence on streamwise surface pressures in separated and reattaching flows. Journal of Wind Engineering and Industrial Aerodynamics 54-55, 313-323.

Nagao, F., Utsunomiya, H., Yoshioka, E., Ikeuchi, A., Kobayashi, H., 1997. Effects of handrails on separated shear flow and vortex-induced oscillation. Journal of Wind Engineering and Industrial Aerodynamics 69-71, 819-827.

Piña, R.B., Caracoglia, L., 2009. Extraction of Flutter Derivatives from Small-Scale Wind Tunnel Experiments, 11th Americas Cnference on Wind Engineering, San Juan, Puerto Rico. 
Sato, H., Hirahara, N., Fumoto, K., Hirano, S., Kusuhara, S., 2002. Full aeroelastic model test of a super long-span bridge with slotted box girder. Journal of Wind Engineering and Industrial Aerodynamics 90, 2023-2032.

Schewe, G., Larsen, A., 1998. Reynolds number effects in the flow around a bluff bridge deck cross section. Journal of Wind Engineering and Industrial Aerodynamics 74-76, 829-838.

Trein, C., Shirato, H., Matsumo), M., 2013. Effects of the Gap Length on the Unsteady Pressure Characteristics of Two-Box Girders 12th Americas Conference on Wind Engineering (12ACWE), Seattle, Washington USA.

$\mathrm{Wu}, \mathrm{T}$., Kareem, A., 2012. An overview of vortex-induced vibration (VIV) of bridge decks. Frontiers of Structural and Civil Engineering 6, 335-347.

Yung, Y., GE, Y., 2008. Aerodynamic Investigation on a long-span suspension bridge with central slotted box girder, 17th Congress of IABSE, Chicago, USA. 


\section{CHAPTER V}

DEVELOPING AN ELASTIC SUSPENSION TEST RIG FOR SECTIONAL MODEL TESTING AT HIGH RE 


\section{CHAPTER V}

\section{DEVELOPING AN ELASTIC SUSPENSION TEST RIG FOR SECTIONAL MODEL}

\section{TESTING AT High RE}

\subsection{Abstract}

In this chapter, the requirements to develop a new two-degree of freedom test rig for studying the wind effects on large scale section models in Wall of Wind (WOW) at Florida International University (FIU) is provided are examined. The objective is to design a test rig which can be used to study a wide range of aerodynamic effects on bridge decks including vortex induced vibration, flutter and buffeting. The novelty of this test setup is that it can be used to study large models at high wind speeds so that the testing at high Reynolds $(R e)$ is possible. Testing at a larger scale is also beneficial to modeling more detailing so that the geometry is closer to the prototype. This chapter gives the details of designing a system that enables simultaneous vertical and torsional motions of the vibrating model. Building such a dynamic test rig is desirable as it can be used to evaluate aeroelastic effects on numerous structures including bridge decks, light poles and etc. The design is based on a sectional rig that is composed of a rigid model and a set of springs from which the model is suspended. By suspending the model from a set of springs, the vibration frequencies of the system can be tuned with changing the number of springs and the spring stiffness. It is also possible to use such a system for performing static test on the model by changing the setup slightly. In that case, only the interaction of the wind and section would be considered without taking into account the self-excited forces generated due to structural motion. The challenges of designing such a system are described here in detail. With the current test setup, the system cannot be used 
for performing vortex induced vibration tests. The functional wind speeds of Wall of Wind dictates a very complicated test setup as the model should be very rigid and large but lightweight at the same time. Also, the vibrating mechanism including the springs and model to springs connections should be very stiff and lightweight also. The system can become functional by reducing the Wall of Wind workable wind speeds, using large rated springs and building a lightweight but rigid model at the same time.

Keywords: Sectional model testing, Elastic suspension system, Aerodynamic similarity

\subsection{Introduction}

Wind-induced loads resulting from the complex interaction of the wind and structure can lead to large amplitude vibrations in flexible structures, e.g. long span bridges. These loads can be destructive and result in total failure of the structure as seen in the Tacoma Narrows Bridge in 1940. Wind effects on structures can be classified into two groups: aerodynamic effects and aeroelastic effects. The aerodynamic effect can be considered as the direct effect of the oncoming wind and its interaction with the structure. The aerodynamic effects of the wind on the structure can be evaluated using a motionless sectional model under the simulated winds. The aeroelastic effect is generated from the wind effects on the structure in motion wherein aerodynamic forces and structural motions interact significantly. Aeroelastic instability may occur if the initial deflection of the structure under the approaching winds creates the basis for divergently increasing deflections. The wind-induced loads which are generated due to the structural motion are termed as self-excited forces. 
In spite of the fact that analytical and numerical simulations have advanced a lot during recent years, they are still incapable of predicting the accurate response of large bluff structures (such as bridges) under turbulent wind loadings without some sort of inputs from experiments. Therefore, the experimental setup remains as a highly advantageous tool to study the complex effects of wind flow on a structure in motion. Sectional model testing is the most common type of wind tunnel testing that is widely used due to its simplicity and proven ability to predict the wind effects on structures Scanlan and Tomko (1971) were the first to introduce the concept of aerodynamic derivatives to study the instability of bridges and they used sectional model testing to collect these derivatives. In a section model testing, a geometrically scaled model of the structure is tested in a wind flow simulating the scaled atmospheric flow conditions. A section model is a two dimensional (2-D) section of the body that is built to a scale to replicate a representative span-wise section of the prototype. The sectional model should be rigid so that the model mode shape remains uniform over its entire length. Sectional model testing can be used both to study the aerodynamic and aeroelastic response of the structure by preventing and allowing the model vibration accordingly. In order to accommodate the model motion for studying the aeroelastic effects, the model is often supported by springs at the ends allowing rigid motions along vertical, torsional and lateral directions.

The sectional model testing is particularly a useful tool to study the aerodynamic stability of the cross section shape in the initial design phase of the prototype. After ensuring the stability of the proposed cross sectional shape, the application of the sectional model testing can be further expanded to evaluate the static and dynamic forces 
expected on the prototype deck. Sectional model testing often preferred to the other types of the wind tunnel testing, i.e. the taut strip model testing and full aeroelastic model testing, as it requires less complex models and is less expensive and faster compared to the other methods. It can also be used to study the wind effects on a wide range of structures including airplane wings, bridge decks, light poles, tall buildings and any other structures for which studying a 2-D portion of its span length can be representative of the entire length.

The current methods for designing bridges considering wind effects depend heavily on the application of flutter derivatives. Flutter derivatives are used to describe the aeroelastic characteristics of the bridge deck which can be obtained from sectional model testing under simulated wind conditions using a forced or free vibration test rig. Forced vibration technique was developed by Kawashima et al (1963) and was applied to bridges by Ukeguchi and Sakata (1965) to study aeroelastic instability. In forced vibration methods, the sectional model is excited sinusoidally in the required degree of freedom with fixed amplitude and frequency using an external actuator. The windinduced loads are measured at supports by comparing the results from the no wind condition to the condition that wind is blowing. The flutter derivatives are obtained from the forced vibration method by identifying the phase lag between the model displacement time history and the aerodynamic load time history and using the data in the relevant algorithms. The evaluation of flutter derivatives is simpler from the forced vibration method compared to the free vibration method. However, due to the complexities associated with building a test setup to force the model motion, the free vibration technique is preferred. 
The free vibration method was first used by Scanlan and Tomko (1971) to experimentally obtain the flutter derivatives. In free vibration methods, the sectional model is usually suspended by a series of elastic springs to permit the sectional model to move with specified vertical and torsional modal frequencies. The sectional model is displaced to an initial position and is released to vibrate freely at a fixed wind speed for a given time period while the displacements are measured. Extracting the flutter derivatives from free vibration methods requires implementing a system identification process on the displacement time histories that are recorded at a number of wind speeds. Several methods have been proposed by different researchers during the years to improve the system identification procedure (Brownjohn and Jakobsen, 2001; Chowdhury and Sarkar, 2004; Gu et al, 2000; Sarkar et al, 1994).

Sectional model testing can also be used to study the self-limited vibrations caused by vortex induced vibration (VIV) (Laima et al, 2013; Larsen and Wall, 2012) in addition to finding the flutter derivatives for evaluating stability. Due to the limitations in the size and the maximum wind speeds that conventional wind tunnels can generate, wind tunnel tests are often performed at $R e 100$ to 1000 times smaller than the prototype $R e$. Recent literature (Larsen et al, 2008; Schewe and Larsen, 1998) showed that results from conventional wind tunnel testing at low Reynolds number $(R e)$ can be different from what is expected on the prototype at higher $R e$. It is therefore of interest to conduct wind effect experiments in a higher $R e$ regime which is closer to the prototype $R e$ range.

In this chapter, the requirements to develop a large scale testing facility are examined with a view to using sectional model tests at higher Re. A design approach for an experimental setup which is capable of performing free vibration sectional model 
testing in the Wall of Wind (WOW) open jet facility at Florida International University (FIU) is given here. WOW is a large open jet facility with a 6.1 wide $\times 4.3 \mathrm{~m}$ high test section which can generate up to a Category 5 Saffir-Simpson Scale hurricane wind speed (more than $240 \mathrm{~km} / \mathrm{hr}$ ). The large size of the WOW test section and the high wind speeds that it can generate provide the opportunity to test larger sectional models under large wind speeds so that the mismatch in the $R e$ can be minimized. A design approach for a test rig which can be used both for studying the VIV and extracting the flutter derivatives from 2-D large sectional models is given here and the challenges for making it to become functional are described in detail. The uniqueness of this test rig is related to the large size of the wind generating facility and the large generated wind speeds that makes it viable to study the wind effects on numerous structures including bridges, light poles and etc. at high $R e$ comparable to prototype structure.

\subsection{Methodology}

In order to study the response of a real structure in laboratory using a scaled model, similarity requirements should be satisfied between the model and prototype. The model should be similar to the prototype geometrically, kinematically and dynamically. It is essential that the geometrical shape of the cross-section of the prototype structure be modeled in the sectional model with all the detailing. Even slight modifications in the cross-sectional shape, particularly at the upwind edge, can result in significant changes in the aerodynamic behavior. Kinematic similarity necessitates achieving the same flow regimes between the model and the prototype. Also the dynamic characteristics of the 
model should be representative of the prototype structure dynamic behavior, i.e. damping and stiffness.

To ensure that the observed response on the model is representative of the prototype structure, several non-dimensional parameters should be equal between the model and the prototype. Froude number (Fluid inertia force/Gravity force), Cauchy number (Elastic force/Fluid inertia force), Reynolds number (Fluid inertia force/Fluid viscous force), Density parameter (Inertia force of structure/Fluid inertia force) and Damping ratio (Damping/Critical damping) are the non-dimensional parameters that need to be equal between the scaled model and prototype so that the results from wind tunnel testing can be representative of the actual response of the prototype. Wind loads and resulting dynamic responses measured from sectional model testing, satisfying the scaling rules, can be analytically extended to predict the response of the prototype structure.

With sectional models, gravitational forces do not affect the model dynamics. Therefore velocity scaling can be selected arbitrarily rather than by respecting the Froude Number (Wardlaw, 1980). Table 1 gives a summary of the parameters that need to be equal between the model and prototype for sectional model testing.

Table 1: Table 1 Scaling rules for sectional model testing

\begin{tabular}{|l|c|}
\hline Geometry & Cross-sectional shape and detailing \\
\hline Mass parameter & $\frac{m}{\rho B^{2}}, \frac{I}{\rho B^{4}}$ \\
\hline Elastic parameter & $\frac{U}{f_{h} B}, \frac{U}{f_{\alpha} B}$ \\
\hline Structural damping & $\xi_{h}, \xi_{\alpha}$ \\
\hline Frequency parameter & $\frac{f_{\alpha}}{f_{h}}$ \\
\hline
\end{tabular}


Where, $U$ is the wind speed $(\mathrm{m} / \mathrm{s}), \rho$ is air density $\left(\mathrm{kg} / \mathrm{m}^{3}\right), B$ is the width of bridge deck (m); $f_{h}$ and $f_{\alpha}$ are vertical and torsional frequencies $(\mathrm{Hz}) ; m$ and $I$ are mass and mass moment of inertia per unit length $\left(\mathrm{kg} . \mathrm{m}^{2} / \mathrm{m}\right) ; \xi_{h}, \xi_{\alpha}$ are vertical and torsional damping ratios.

Scruton number $(\mathrm{Sc})$, is an important non-dimensional parameter for vibration problems which controls the amplitudes of vibration generated due to vortex induced vibration (Wardlaw et al, 1983). Scruton number is a joint function of the mass parameter and structural damping and can be obtained from the following equation:

$$
S c=\frac{4 \pi m \xi}{\rho B^{2}}
$$

The response of the prototype deck due to vortex induced vibration can be estimated from sectional model testing as long as the similarity requirements are satisfied and the simulated wind flow is representative of the actual wind flow that the prototype deck experiences. In order to study vortex induced vibration using a scale model, the testing wind speed, frequency of the motion and model width should be selected so that the reduced velocity $(U / f B)$, corresponding to vortex induced vibration in the prototype, can be achieved in the experiments. In the context of bridges, vortex induced vibration usually occurs at low to moderate wind speeds on the prototype. In a wind tunnel study, either the testing wind speed should be very small or the frequency of the motion should be very large so that the range of small reduced velocities needed for vortex induced vibration studies can be achieved. 
Self-exited forces generated due to the structural motion in a wind flow can be obtained from the following equations along the vertical and torsional degrees of freedom (Scanlan, 1978):

$$
\begin{aligned}
L_{h} & =\frac{1}{2} \rho U^{2} B\left[k H_{1}{ }^{*}(k) \frac{\dot{h}}{U}+k H_{2}{ }^{*}(k) \frac{B \dot{\alpha}}{U}+k^{2} H_{3}{ }^{*}(k) \alpha+k^{2} H_{4}{ }^{*} \frac{h}{B}\right] \\
M_{\alpha} & =\frac{1}{2} \rho U^{2} B^{2}\left[k A_{1}{ }^{*}(k) \frac{\dot{h}}{U}+k A_{2}{ }^{*}(k) \frac{B \dot{\alpha}}{U}+k^{2} A_{3}{ }^{*}(k) \alpha+k^{2} A_{4}{ }^{*} \frac{h}{B}\right]
\end{aligned}
$$

where, $k$ is the reduced frequency $(2 \pi f B / U), h$ and $\alpha$ are the vertical and torsional displacements, over-dot indicates the derivatives with respect to time and $H_{i}{ }^{*}$ and $A_{i}{ }^{*}(i=1$ to 4$)$ are flutter derivatives.

To acquire the flutter derivatives, used in the stability analysis of the bridge decks, free vibration sectional model testing is often performed in a smooth flow condition. Flutter derivatives are normally obtained over a wide range of nondimensional reduced velocity $(U / f B)$. The maximum testing wind speed should be higher than the critical velocity for the studied aerodynamic instability phenomenon (i.e. critical flutter velocity). The desired natural frequency of the system for the vertical and torsional degrees of freedom can be obtained from the highest reduced velocity that needs to be generated for a particular experiment. The required vertical and torsional stiffness of the system can be evaluated from the following equation:

$$
K=\omega^{2} M
$$

where $K$ is the stiffness resulted from the springs, $\omega$ is the desired circular frequency in $\mathrm{rad} / \mathrm{sec}$, and $M$ is the participating mass or mass moment of inertia for a particular degree of freedom. It is to be noted that $M$ denotes the total mass over the entire span while $m$ is the mass per unit length ( $m=M /$ span length). 


\subsection{Experimental Setup Design}

In order to perform wind tunnel testing on sectional models, end plates are typically attached to the two far ends of the model to reduce aerodynamic end effects. The functionality of end plates is to ensure 2-D air flow over the section model. In the previous researches, end plates with different shapes and sizes were used for different cross sectional shapes. For rectangular section models, Haan Jr. et al (1998) used rectangular end plates of height 7 times the model depth which were extended 2.5 and 5 times the model depth from the model's leading and trailing edges, respectively.

In this study, two large streamlined end walls were built and placed in front of WOW to cover the test rig and measurement tools from wind excitations. In addition to encasing the test rig, the end walls performed as large end plates on the two ends of the model. The end walls were anchored to the floor and together using guy wires to prevent motion in the walls. Figure 1(a) shows a view from the upstream of the sectional model installed within the two end walls spaced $3 \mathrm{~m}$ from each other in the test section of WOW. A streamlined shape was adopted for the end walls by introducing a 1:7 slope in the upstream edge so that the flow stays attached to the wall and separation is prevented (see Fig. 1(b)). 


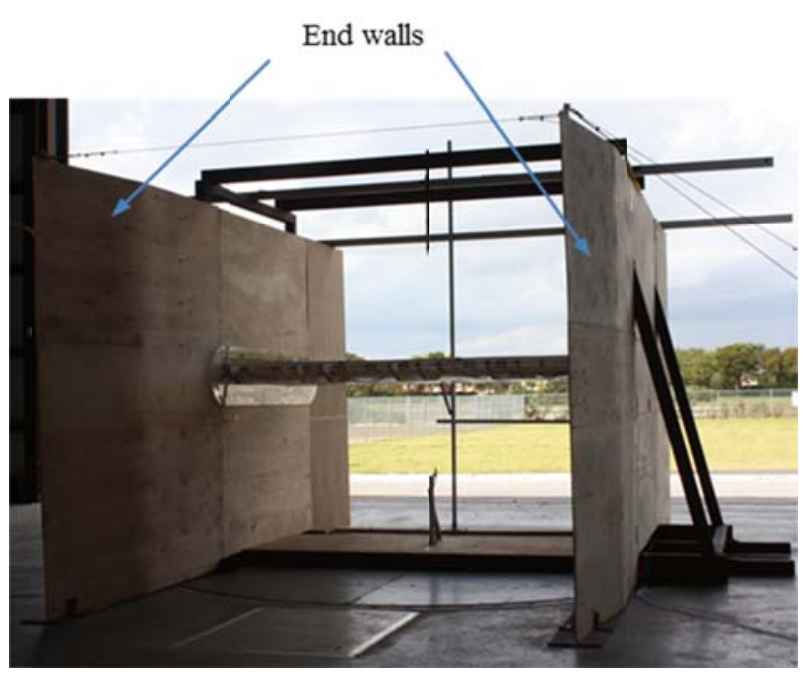

(a) View from the upstream

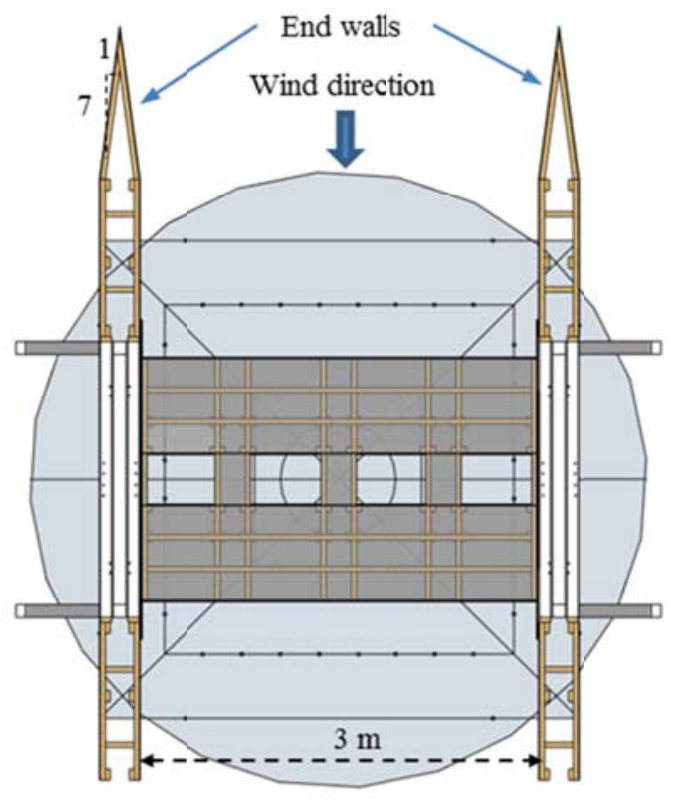

(b) Top view-schematic

Figure 1: End walls

The current test rig was designed to be versatile so that it can be used for both static and dynamic sectional model testing. It can also be used to test models with different span lengths by adjusting the space between the two frames. In the previous chapters this space was set to be equal to 3 meters for studying bridge models with $3 \mathrm{~m}$ span length.

Figure 2 shows an overview of the test rig for dynamic tests. In order to enable the model motion for free vibration sectional model testing, a set of extension springs can be used to allow motions in the vertical and torsional directions (see Fig. 2). The sectional model is suspended from the springs using a connection system consisting of aluminum I-beams, aluminum pipes and two rectangular aluminum plates that are attached to the two ends of the sectional model. Figure 3 shows the outline of the 
connection systems. Three hollow aluminum pipes are welded to the aluminum plate on one end and are connected to the I-beam with nuts and bolts on the other end to facilitate the change of tilt angle. The hole pattern on the aluminum I-beam for changing the angle of attack from $-6^{\circ}$ to $+6^{\circ}$ is shown in Fig.3.

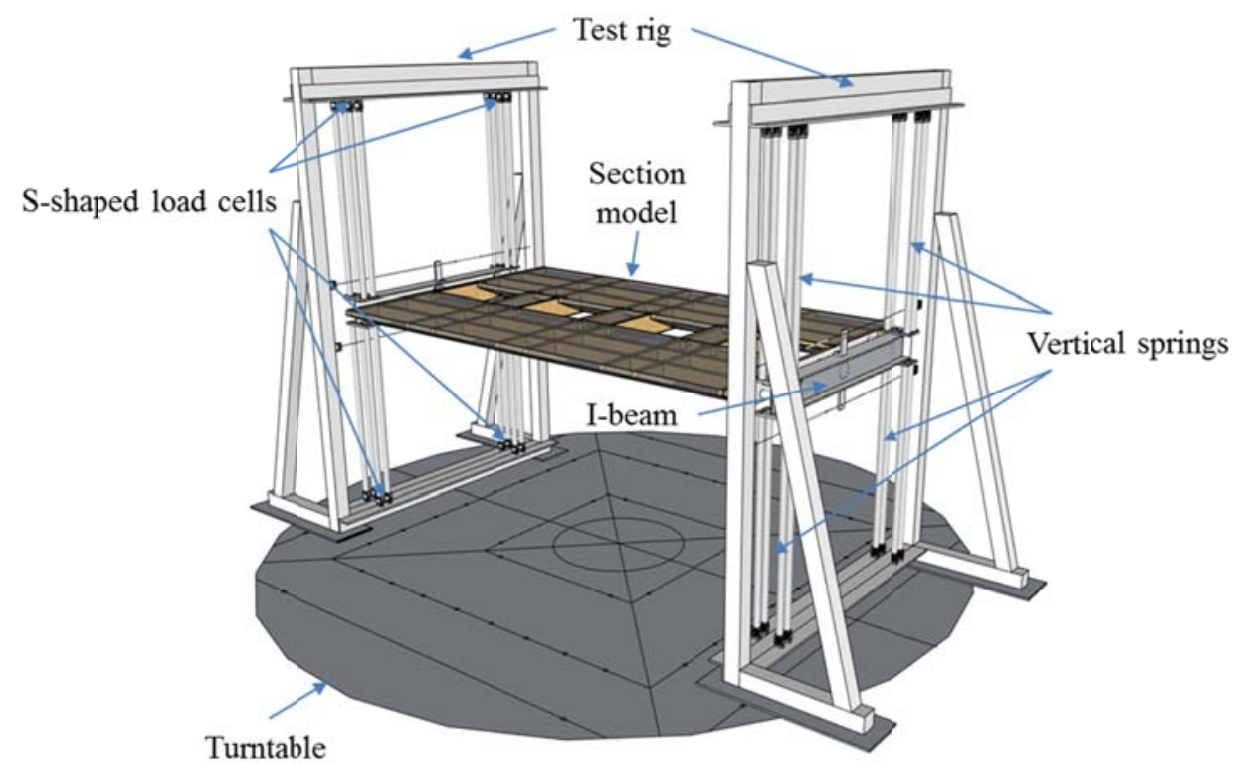

Figure 2: Dynamic test rig

The extension springs are connected to the flanges of the I-beam on one side and to S-shaped load cells attached to the test rig on the other side. The S-shaped load cells are connected to the springs to measure the instantaneous tension force in the springs, generated from weight and wind loads. The tension force in each spring can be converted to the corresponding displacement from the spring stiffness. Figure 4 shows a view of the high-accuracy S-shaped load cells (LCCA) manufactured by Omega company that are used in the current test setup.

The number of springs used in this test setup can change from 8 to 32 springs according to the required stiffness for different purposes. The system stiffness and as a 
result the system frequency can also be modified by using different set of springs with different stiffness to meet a required condition based on the type of test and parameters investigated. Torsional moments generated due to the wind are carried by the pairs of vertical springs that also enable the vertical motions. The frequency of the torsional motion can be adjusted by changing the distance between the two rows of vertical springs in the along wind direction. In-line (horizontal) motions are prevented by using a set of springs and pre-stressed wires connecting the I-beams to the frames in the horizontal direction (see Fig. 2). The pre-stressed wires connect the L-shaped aluminum pieces, attached to the top and bottom flanges of the I-beam, to the vertical members of the frame to prevent the horizontal motion. This configuration restricts the horizontal motion but permits free vibration in the vertical and torsional directions.

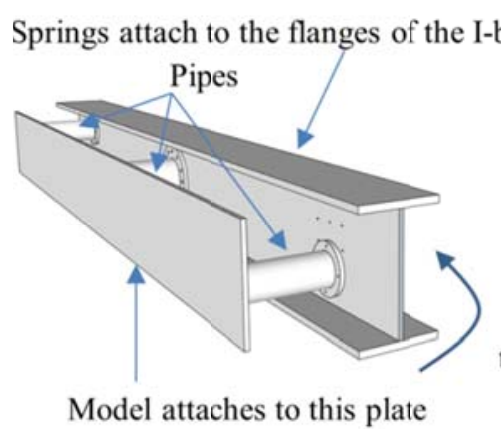

(a) Back view

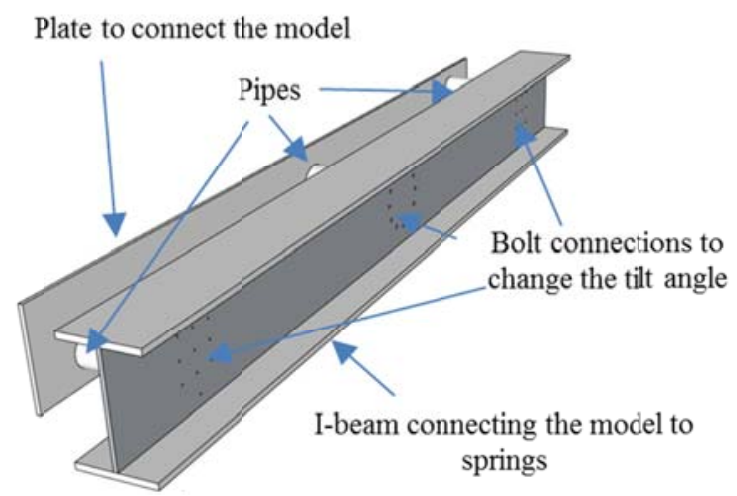

(b)Front view

Figure 3: Sectional model to springs connection system 


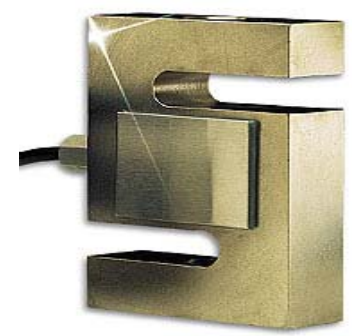

Figure 4: S-shaped load cell

In order to perform static (motionless) tests on sectional models, the springs can be removed from the test rig. Figure 5 shows an overview of the test rig for performing static tests. Four L-shaped steel beams are added to the test setup to provide a support for a fixed connection. A pair of JR3 multi-axis load cells are installed on top of the Lshaped beams on each side as shown in Fig. 5. Finally, the I-beams on the two ends of the models are rigidly fixed to the load cells so that the wind-induced loads can be measured on the sectional model. 


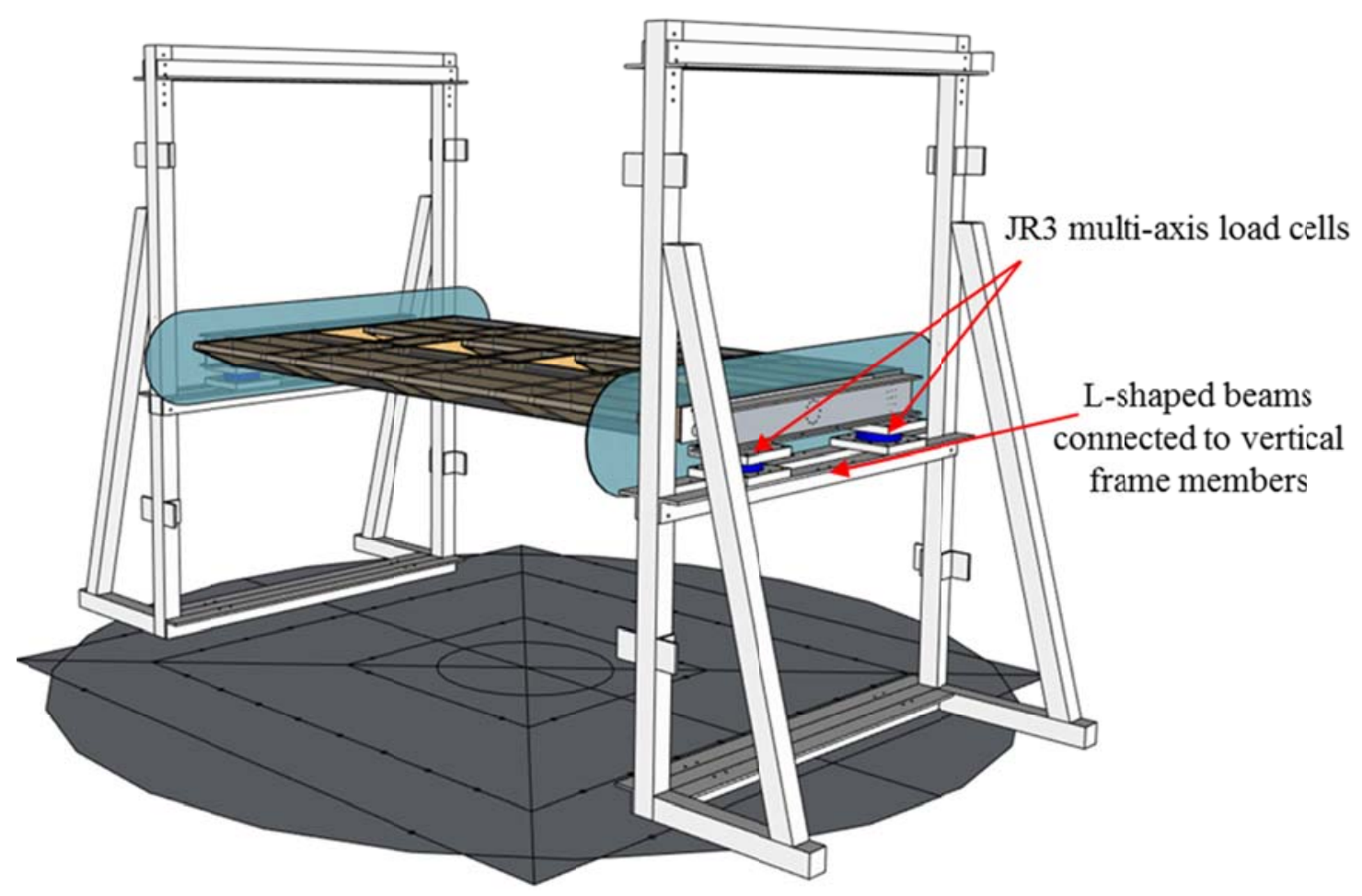

Figure 5: Static test rig

In the following section, the design of the springs (for stiffness and spacing) is given so that the test rig can be used to study aeroelastic effects on a twin box girder bridge at larger $R e$ regime compared to the conventional wind tunnel testing.

The bare cross sectional shape of the eastern span of the San Francisco-Oakland Bridge (East Bay Bridge) deck with the inclusion of the traffic barriers is selected. Analysis of the lift spectra from static tests detected presence of vortex shedding on this section. This bridge features a twin deck configuration carried by self-anchored suspension cables where the two decks are linked together with $10 \mathrm{~m}$ wide transverse beams every $20 \mathrm{~m}$. Figure 6 shows a photograph of the East Bay Bridge which was taken while travelling on the east-bound part of the bridge towards San Francisco. The single deck chord length $(C)$, total width $(B)$ and height $(H)$ of the bridge deck are $28 \mathrm{~m}, 71 \mathrm{~m}$ 
and $5.5 \mathrm{~m}$, respectively (Fig. 7). Table 2 gives the properties of the prototype bridge deck that need to be scaled down according to the scaling rules, given in Table 1, and be simulated in the sectional model testing. The information on the bridge specifications was provided by T.Y. LIN INTERNATIONAL GROUP, which was involved in all phases of developing this bridge, from designing to construction.

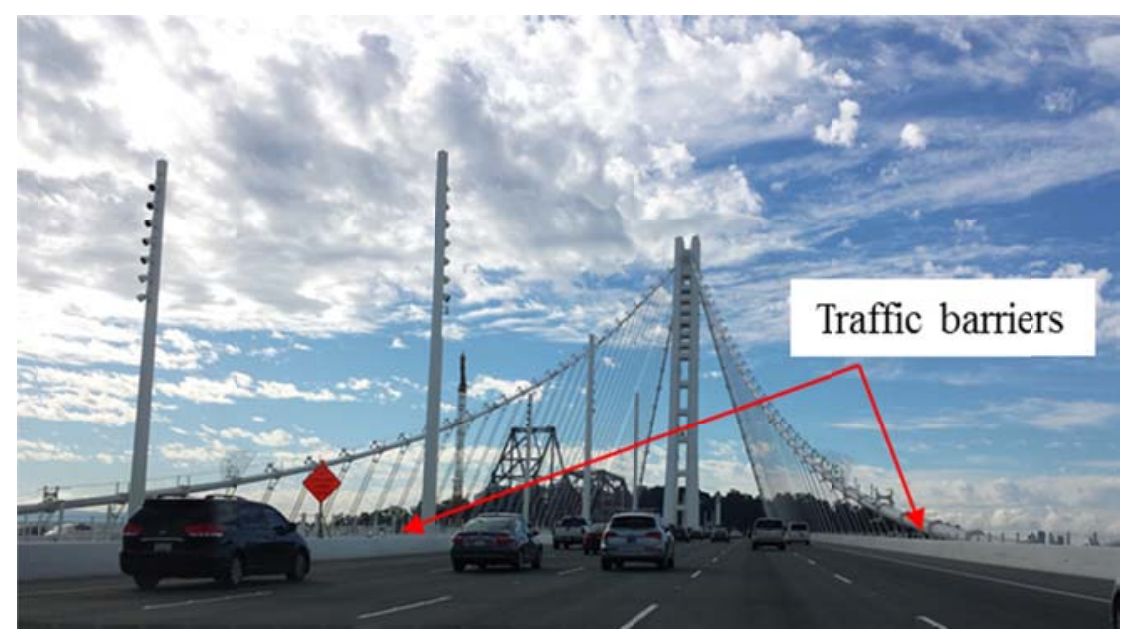

Figure 6: East bay bridge
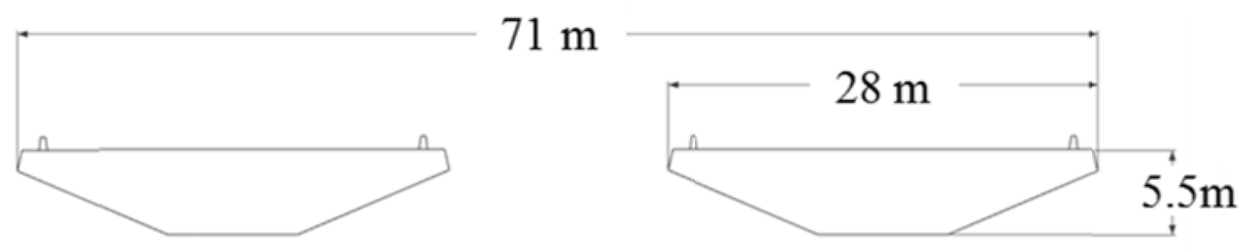

Figure 7: Simplified cross sectional shape of the East bay bridge

Table 2: Properties of the prototype bridge deck

\begin{tabular}{|l|l|}
\hline Mass per unit length $(\mathrm{kg} / \mathrm{m})$ & 61125 \\
\hline Mass moment of inertia per unit length $\left(\mathrm{kg} \cdot \mathrm{m}^{2} / \mathrm{m}\right)$ & 34740000 \\
\hline Critical damping ratio $(\xi)$ & 0.003 \\
\hline Torsional to vertical frequency $\left(f_{\alpha} / f_{h}\right)$ & $0.30 / 0.22 \cong 1.4$ \\
\hline
\end{tabular}


The span length of the model was limited to $3 \mathrm{~m}$ based on the dimensions of the test section and to ensure a uniform wind flow across the span length. According to the literature (Laima et al, 2013; Piña and Caracoglia, 2009), aspect ratios (ratio of the span length to model width) as low as 1.6 are adequate to ensure a 2-D airflow along the section model length which can be representative of the aerodynamic response of the prototype. Requirements of the minimum aspect ratio and limitations in the maximum span length resulted in the selection of the model width to be equal to $1.9 \mathrm{~m}$, equivalent to $1 / 36$ scaling factor for the studied bridge deck. 1/36 scaled model was therefore the largest model of this particular bridge deck that could be tested using WOW. However, models with smaller dimensions could also be studied using the current setup, keeping in mind the lower $R e$ regime that they would represent.

Scaling rules require the equality of the mass parameters between the model and the prototype. Based on the information on the mass, mass moment of inertia and dimensions of the prototype, the mass parameters $m / \rho B^{2}$ and $I / \rho B^{4}$ are calculated to be equal to 9.6 and 1.1 respectively. Therefore, the mass per unit length for a $1 / 36$ scaled model should be equal to $47.2 \mathrm{~kg} / \mathrm{m}$. In order to make a model which satisfies the mass scaling requirement, the rigid model was made out of a wooden frame and was covered by Plexiglas plates to produce the final cross sectional shape (see Fig. 8). The total mass of the sectional model with the inclusion of the connecting equipment (The two I-beams connecting the sectional model to the springs) was measured to be equal to $163 \mathrm{~kg}$, meaning that $m / \rho B^{2}$ was equal to 11.9 on the model which is higher than the targeted value. However, the damping of the vibrating system can be adjusted to meet the target 
$S c$ of the prototype. It is to be noted that the total mass should include the portion of the springs mass which is in motion but was not considered here.

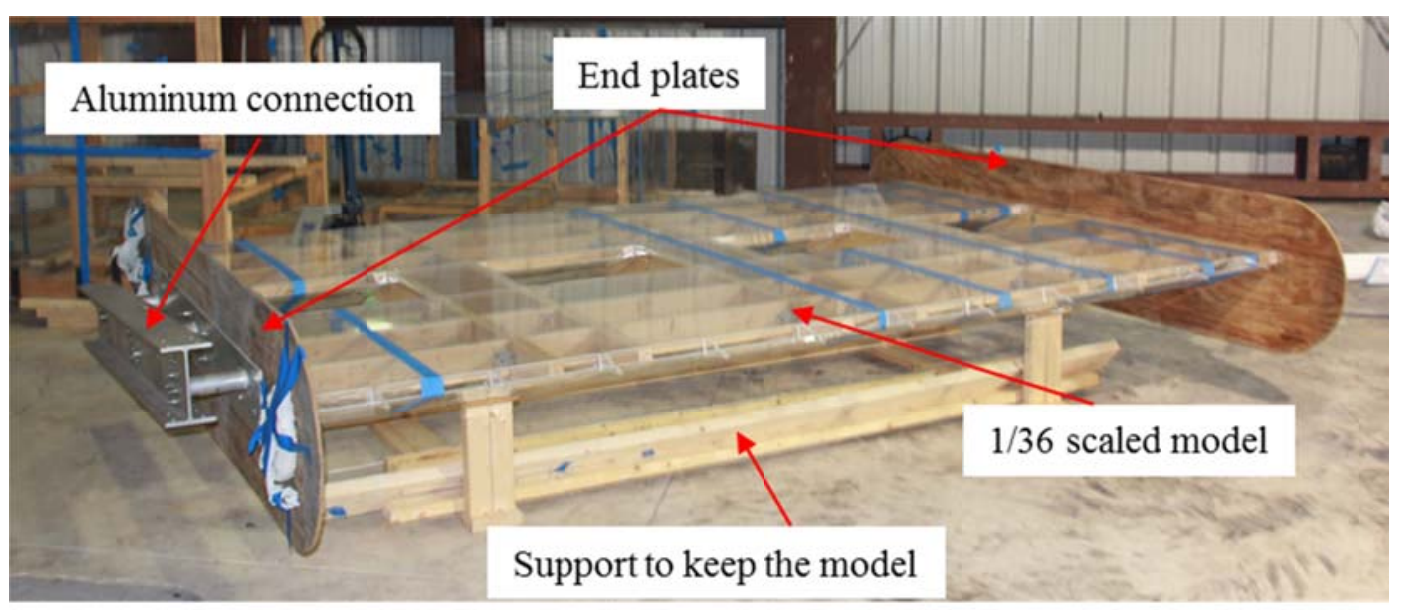

Figure 8: A view of the sectional model

The frequency at which the vortices are shed $(f)$ is proportional to the approaching mean wind speed $(U)$ and inversely proportional to the height $(H)$ of the structure. This is expressed non-dimensionally as the Strouhal number:

$$
S t=\frac{f H}{U}
$$

Spectral analysis on fluctuating lift forces measured on the motionless section model of this bridge section showed that vortex shedding occurs at a Strouhal number (St) range between 0.146 to 0.168 , changing with the Re, for zero angle of attack. Figure 9 shows the variation of the $S t$ with $R e$ for the different wind angles of attack based on the lift measurements on the motionless section model of the studied cross section. 


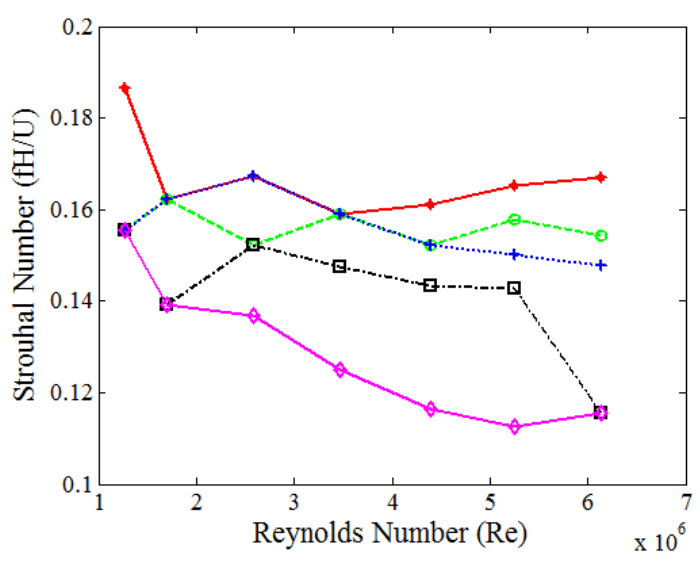

Figure 9: Strouhal number as a function of $\mathrm{Re}$

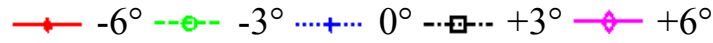

Due to the alternating shedding of vortices, periodic forces are generated that result in structural motion in a plane normal to the direction of wind flow. Significant vibration can occur when the frequency of vortex shedding approaches one of the natural frequencies of the structure. These cross-wind vibrations have a strong organizing effect on the vortex shedding pattern which can increase the strength of the vortices and couple the vortex shedding frequency to the natural frequency of the structure. This phenomenon is known as lock-in. The critical wind speed $\left(U_{c r}\right)$ at which lock-in occurs can be estimated using the Strouhal number relation:

$$
U_{c r}=\frac{f_{h} H}{S t}
$$

where $f_{h}$ is the natural frequency of the structure in the heaving (vertical) direction.

From eq. (4), the total stiffness of the springs in the vertical direction should be equal to $\omega_{h}^{2} M$. It is known that circular frequency in the vertical direction is equal to:

$$
\omega_{h}=2 \pi f_{h}
$$


The springs' total stiffness can be calculated from the combination of eqs. (4) to (7), as:

$$
K=\left(\frac{2 \pi U_{c r} S t}{H}\right)^{2} M
$$

$S t$ is a factor of the cross-section shape and the $R e$ at which the tests are performed. For the given bridge deck, the $S t$ value is adopted as 0.15 , based on the measurements on the static tests. In order to reduce the stiffness of the required springs and use softer springs, one option is to perform tests on larger scaled models (increasing $H$ ). It would also be possible to use softer springs either by decreasing the model weight or performing the tests at smaller wind speeds. For the current test setup, the minimum wind speeds simulating a uniform flow along the span length using WOW was taken as around $10 \mathrm{~m} / \mathrm{s}$. The scale factor of the largest sectional model which can be tested in WOW for the given section was found to be $1 / 36(H=0.15 \mathrm{~m})$. The total mass of the 1/36 scaled model without the inclusion of the moving part of the springs was also measured to be equal to $163 \mathrm{~kg}$. From eq. (8) and the given data, the total stiffness of the springs that can be used to study the vortex induced vibration on the given cross-sectional shape should be larger than $643498.2 \mathrm{~N} / \mathrm{m}$. As a result, the stiffness of each spring should be larger than $20110 \mathrm{~N} / \mathrm{m}$ if a total number of 32 springs is used to suspend the model. It is clear that the stiffness of each spring should be larger than this value as the weight of the moving part of the springs was not included in the calculations. The information on the properties of an extension spring made by Sterling Sprigs L. L.C is given here to demonstrate the spring weight effects on the calculations: 
Table 3: Properties of an extension spring made by Sterling Sprigs L.L.C

\begin{tabular}{|l|l|}
\hline Wire diameter $(\mathrm{mm})$ & 9.2 \\
\hline Spring rate $(\mathrm{N} / \mathrm{m})$ & 18038 \\
\hline Mean diameter $(\mathrm{mm})$ & 33.5 \\
\hline Spring index & 3.6409 \\
\hline Initial free length $(\mathrm{m})$ & 1.0 \\
\hline Maximum deflection $(\mathrm{m})$ & 0.18 \\
\hline Weight $(\mathrm{kg})$ & 5.6 \\
\hline
\end{tabular}

From table 3, the total weight of the springs would be equal to $179.2 \mathrm{~kg}$ (for 32 springs) and if only $1 / 3$ of the springs' weight gets involved in the motion, the total mass that needs to be considered for the spring calculations would be around $222.7 \mathrm{~kg}$. A second iteration on the required stiffness of springs from eq. (8) results in $879316 \mathrm{~N} / \mathrm{m}$ for the total of 32 springs, meaning that the stiffness of each spring should be larger than $27478.6 \mathrm{~N} / \mathrm{m}$. One alternative to use softer springs is to reduce testing wind speeds. For example, by reducing the range of testing wind speed by a factor of 2 , springs which are 4 times softer can be used. WOW is an open jet facility and such condition can be achieved by performing the tests at times of the day that natural winds are small enough so that they don't interfere with the test results.

A similar procedure can be followed to obtain the required spring stiffness to perform tests for flutter derivative identification. The only difference is that for this purpose, the maximum reduced velocity that needs to be simulated is the factor that determines the stiffness. From the reduced velocity equation:

$$
f_{h}=\frac{U}{\text { reduced velocity } \times B}
$$

From Eq. (9) it is seen that the frequency of motion is a factor of the testing wind speed. A larger testing wind speed is of interest to capture the effect of Re. For example, 
if the maximum reduced velocity that needs to be simulated is equal to 7 and considering the maximum wind speed that WOW can generate (around $67 \mathrm{~m} / \mathrm{s}$ ), the frequency of motion $f_{h}$ that needs to be simulated for the $1 / 36$ scaled model would be equal to $5 \mathrm{~Hz}$. From Eqs. (4) and (7), the total springs stiffness should be larger than $163302 \mathrm{~N} / \mathrm{m}$.

\subsection{Summary}

This document summarizes the methodology to develop a dynamic sectional model test rig that can be used in studying the vortex induced vibration and/or flutter derivatives identification of structures at high $R e$ regime. The current test setup would be capable of studying other aerodynamic and aeroelastic phenomena like across-wind galloping, rain-wind induced cable vibration, torsional divergence, buffeting, etc. The sectional model would be suspended from a set of extension springs to accommodate motions in the vertical and torsional degrees of freedom. The properties of the springs can be designed based on the type of the aeroelastic phenomena under study. In this document, the spring design is given for studying vortex induced vibration and extraction of the flutter derivatives for a twin box girder bridge deck under the high wind speeds of WOW. The forces generated in the springs due to the wind loads would be measured and converted to displacements knowing the springs' stiffness. In the case that all the scaling rules and Scruton number equality are satisfied, the prototype response due to vortex induced vibration can be calculated by multiplying the measured response on the model by the scaling factor. The extracted flutter derivatives can also be used to obtain the wind speed associated with instability on the prototype. 


\subsection{References}

Brownjohn, J.M.W., Jakobsen, J.B., 2001. Strategies for aeroelastic parameter identification from bridge deck free vibration data. Journal of Wind Engineering and Industrial Aerodynamics 89, 1113-1136.

Chowdhury, A.G., Sarkar, P.P., 2004. Identification of eighteen flutter derivatives of an airfoil and a bridge deck. Wind and Structures 7, 187-202.

Gu, M., Zhang, R., Xiang, H., 2000. Identification of flutter derivatives of bridge decks. Journal of Wind Engineering and Industrial Aerodynamics 84, 151-162.

Haan Jr., F.L., Kareem, A., Szewczyk, A.A., 1998. The effects of turbulence on the pressure distribution around a rectangular prism. Journal of Wind Engineering and Industrial Aerodynamics 77-78, 381-392.

Kawashima, S., Kimura, H., Shibato, T., 1963. An experiment for unsteady aerodynamic forces acting on a wing, Proceedings of 13th Japan National Congress for Applied Mechanics, Tokyo, Japan, pp. 19-20.

Laima, S., Li, H., Chen, W., Li, F., 2013. Investigation and control of vortex-induced vibration of twin box girders. Journal of Fluids and Structures 39, 205-221.

Larsen, A., Savage, M., Lafrenière, A., Hui, M.C.H., Larsen, S.V., 2008. Investigation of vortex response of a twin box bridge section at high and low Reynolds numbers. Journal of Wind Engineering and Industrial Aerodynamics 96, 934-944.

Larsen, A., Wall, A., 2012. Shaping of bridge box girders to avoid vortex shedding response. Journal of Wind Engineering and Industrial Aerodynamics 104-106, 159-165.

Piña, R.B., Caracoglia, L., 2009. Extraction of Flutter Derivatives from Small-Scale Wind Tunnel Experiments, 11th Americas Cnference on Wind Engineering, San Juan, Puerto Rico.

Sarkar, P.P., Jones, N.P., Scanlan, R.H., 1994. Identification of Aeroelastic Parameters of Flexible Bridges. Journal of Engineering Mechanics 120, 1718-1742.

Scanlan, R.H., 1978. The action of flexible bridges under wind, I: Flutter theory Journal of Sound and Vibration 60, 187-199.

Scanlan, R.H., Tomko, J.J., 1971. Airfoil and Bridge Deck Flutter Derivatives. Journal of the Engineering Mechanics Division 97, 1717-1737.

Schewe, G., Larsen, A., 1998. Reynolds number effects in the flow around a bluff bridge deck cross section. Journal of Wind Engineering and Industrial Aerodynamics 74-76, 829-838. 
Ukeguchi, N., Sakata, H., 1965. An investigation of aeroelastic instability of suspension bridge. Journal of the Japan Society for Aeronautical and Space Science 133, 27-36.

Wardlaw, R.L., 1980. Sectional versus full model wind tunnel testing of bridge road decks. Proc. Indian Acad. Sci. (Engg. Sci.) 3, 177-198.

Wardlaw, R.L., Tanaka, H., Utsunomiya, H., 1983. Wind tunnel experiments on the effects of turbulence on the aerodynamic behaviour of bridge road decks. Journal of Wind Engineering and Industrial Aerodynamics 14, 247-257. 


\section{CHAPTER VI}

SUMMARY AND CONCLUSIONS 


\section{CHAPTER VI}

\section{SUMMARY AND CONCLUSIONS}

The work done in this dissertation has evaluated the effect of flow characteristics, i.e. $R$ e and turbulence characteristics and geometry details on the aerodynamic response of a twin deck bridge through a set of experiments performed at the WOW. The cross sectional shape of the case study was adopted based on the simplified cross section of the San Francisco/Oakland Bay Bridge. The motivation behind this study was the limited information available on the effect of the aforementioned parameters on the aerodynamic response of double deck bridges, which are gaining popularity due to the needs of increasing traffic. The effect of these parameters was tested on a range of aerodynamic features such as chord-wise pressure distribution, force/moment coefficient and their derivatives with respect to angle of attack, and lift spectra. Due to the limited size of wind tunnels, it is not possible to simulate $R e$ and turbulence integral length scale corresponding to the prototype condition in wind tunnel studies. Also, it is hard to model all the shape details on a small scale model of a bridge section. Results from this study identify the sensitivity of the aerodynamic response of double deck bridges to the unavoidable approximations associated with simulating wind and structure in a wind tunnel study: i.e. $R e$, turbulence and shape details. As a result, this information can be used to assist design of double deck bridges. The results also include information on the efficacy of vortex mitigation devices at different $R e$ regimes. This document is first of its kind to evaluate the $R e$ effects on efficiency of vortex generators in suppressing vortex shedding in double deck bridges. The effect of gap width was investigated by testing models with different gap to bridge with ratios. This information can help designers to 
adopt gap widths so that the prototype bridge performs better against aerodynamic instabilities. The conclusions from this study can be summarized and categorized into three groups, as follows:

\subsection{Re Number Effects}

In order to evaluate $R e$ effects on aerodynamic characteristics, a 1/36 scaled model of the section was tested in a nominally smooth flow with turbulence intensity around $3 \%$. The pressure distribution and aerodynamic forces were measured over a Reynolds number $(R e)$ range from $1.3 \times 10^{6}$ to $6.1 \times 10^{6}$ based on the deck width by changing the testing wind speed. The studied bridge section showed different behavior with regards to the $R e$ sensitivity for different wind angles of attack, showing higher dependency on Re for positive angles of attack. The highest sensitivity was observed at zero and $+3^{\circ}$ wind angles of attack, for which the pressure distribution changed considerably within the gap region with $R e$ increase. From the force measurements, it was observed that for zero angle of attack, which is the most important case, the maximum drag coefficient change was noticed to be around $8 \%$. Thelift coefficient changed more than $70 \%$, for the change in Re values simulated, but since its absolute value is small at zero angle of attack this is probably not so important in practice as the drag coefficient change. Based on quasi steady assumptions and the measured force coefficients, it can be conclude that testing at lower Re regime is conservative for this deck cross-section for aeroelastic analysis of flutter, particularly at positive wind angles of attack. It was also noticed that Re increase helped in sharing loads more uniformly between the two girders. The peak observed in the lift spectra showed that this section was susceptible to vortex shedding. Vortex shedding of this twin-deck bridge was mainly 
governed by the vortex shedding from the trailing edge of the upstream deck and its impingement on the downstream deck. Adding a pair of diverging vortex generators to the bottom surface of the girders was effective in mitigating the vortex shedding, particularly at lower Re values.

\subsection{Turbulence Effects}

Two $1 / 36$ and $1 / 72$ scaled models of the section were tested statically under two flow conditions with nominal $3 \%$ and $6 \%$ turbulence intensities. The effect of turbulence integral length scale for a given turbulence intensity was studied by comparing the results from the two different scaled models, meaning that the ratio of the length scale to the bridge width on the smaller model was twice that on the larger model. Based on the results, with increasing the turbulence intensity, the peaks of the negative mean pressure distribution on the top surface of the upstream deck moved closer to the leading edge and the distribution got narrower on either side of the peak, for all the different wind angles of attack. Comparing the results from the two different scaled models in a given flow condition, it was noticed that smaller integral length scale to bridge width ratio resulted in the larger mean negative pressures near the transition points on the bottom surfaces. The effective forces and moment per unit length were calculated by integrating the measured chord-wise surface pressures over their effective length. From the calculated force/moment coefficients, it can be seen that the steady force/moment coefficients showed sensitivity to the turbulence characteristics as well as $R e$, with the largest influence attributed to the turbulence intensity. The steady force/moment coefficients showed a linear relationship with the reduced turbulence intensity. Analysis of the fluctuating lift spectra indicated that larger turbulence intensity had a mitigating effect on 
the vortex shedding, as with the turbulence intensity increase, the vortex shedding induced lift was reduced. Larger vortex shedding induced loads were also observed for larger turbulence integral length scale to bridge width ratios. The effect of turbulence characteristics was also investigated on the buffeting loads. The aerodynamic admittances, obtained from the lift and wind components spectra, was larger for the larger turbulence integral length scale to bridge width ratios. Analysis of the span-wise distribution of the wind loading showed that for a given separation length, the cross correlation of the wind loads was larger for the larger model, showing the limitations associated with the strip assumption in defining buffeting loads.

\subsection{Shape Details Effects}

Three different gap to single deck width ratios of $0.5,0.75$ and 1.0 were considered to evaluate the effect of gap width. It was observed that changing the gap width had a significant effect on the pressure distribution around the downstream deck, particularly for the smaller wind angles of attack. Based on the pressure distribution data, it can be hypothesized that the wind flow was ventilated through the gap for the smallest gap width. However, for the larger gap widths, the downstream deck was immersed in the wake of the upstream deck for the largest gap width. The force and moment coefficients showed a limited sensitivity to the gap width with an increase in the drag and a decrease in the lift. The pattern of the variation of the force and moment coefficients with respect to the angle of attack was almost similar for all cases. From the spectral analysis of the lift forces, it can be seen that for the zero and $+3^{\circ}$ wind angles of attack, an increasing trend was observed for the Strouhal number with the gap width increase. It was also 
observed that vortex shedding was more pronounced around the downstream deck and at a larger frequency compared to the upstream deck.

The effect of the deck furniture including the traffic barriers and an attached bike path was studied by including these features in the model with the smallest gap width. The results showed that the mean pressure distribution was significantly influenced by the inclusion of the traffic barriers. The traffic barriers not only changed the local pressures on the top surface, but also they increased the negative pressure over the bottom surface, due to the blockage effect on the top surface. The pressure modifications due to the traffic barriers and the direct effect of the wind on the barriers resulted in the formation of a larger lift and drag. Between the aerodynamic coefficients, only the derivative of the drag coefficient with respect to the angle of attack showed sensitivity to the inclusion of the traffic barriers. The mean pressure distribution and the measured loads showed little sensitivity to the inclusion of the bike path and appendages in the simulation of the cross sectional shape. It was observed that with the addition of the traffic barriers and the bike path to the bare deck section, vortex shedding was not greatly affected. 
CHAPTER VII

RECOMMENDATIONS FOR FUTURE RESEARCH 


\section{CHAPTER VII}

\section{RECOMMENDATIONS FOR FUTURE RESEARCH}

In this study, the effect of flow parameters (i.e. $R e$ and turbulence characteristics) and shape details were investigated on the aerodynamic response of twin deck bridges. The objective of this dissertation was to increase the accuracy of the wind simulations for bridge experiments by pointing out the effects of aforementioned parameters on the aerodynamic response of twin deck bridges. In this section some recommendations for further research on the work performed in this dissertation are listed. Recommendations for future work include expanding the parameter ranges of the current project (e.g. Re range, turbulent flow conditions, model dimensions and shape details) and improving the facilities that enable dynamic testing for studying the aeroelastic response of models. The recommendations for future research are described in the following sections.

\subsection{Dynamic Tests at High Re to Study Aeroelastic Response}

In this dissertation, the effects of high $R e$ were evaluated on the aerodynamic response of a twin-deck bridge by testing scaled models in static condition. The design requirements for a two-dimensional dynamic test rig were provided in Chapter V. The dynamic test rig can be used to study the aeroelastic response of the studied cross section due to vortex induced vibrations. The interaction of the wind-induced loads and the structural motions can be simulated using the mentioned test rig to further highlight the effect of structural motions on the bridge responses. The efficiency of vortex mitigation devices can also be evaluated at high $R e$ to investigate how the structural motions can amplify or mitigate vortex shedding around the section. Flutter derivatives can also be measured using this dynamic test rig by introducing an initial condition and allowing free 
vibrations to investigate the effect of $R e$ on twin-deck bridges instability. With the current test setup available, particularly the heavy weight of the model and the limit on minimum wind speeds that the WOW can generate, the system is not functional. Further research needs to be done to make the system functional and available for performing dynamic tests.

\subsection{Comparison of Experimental Results with Analytically Obtained Results}

The vortex shedding generated loads can be represented using sinusoidal forces that are related to the fluctuating lift coefficient due to vortex shedding and vortex shedding frequency which can be calculated from the Strouhal number. These parameters were measured in this study using static sectional models. The aeroelastic response of the prototype bridge can be estimated by modeling the bridge using a Finite Element Method software and applying the vortex shedding loads by a simple harmonic load as described. The results from this numerical analysis can be compared to the results of the dynamic tests to investigate the accuracy of the simple analytical method.

\subsection{Investigating the Effect of Additional Shape Details}

In this study the effects of vortex mitigation devices, gap width, traffic barriers and bike path were evaluated on the aerodynamic response of the studied twin-deck bridge. In order to extend knowledge on the effects of shape details on the bridge aerodynamic response, the effect of the following appurtenances could also be evaluated:

1. Railings: According to the results of this study, traffic barriers played an important role on the vortex shedding from the studied bridge section. It is therefore of interest to study the effects of edge railings and their details on the vortex induced vibrations and stability at high $R e$. 
2. Vortex mitigation devices: In this study, the effects of vortex generators composed of two converging rectangular plates attached to the bottom surfaces of the models were evaluated at high $R e$ regime. Several other types of vortex mitigation devices including guide vanes can also be evaluated at high Re. This can help to choose proper vortex mitigating devices that are effective at prototype condition.

3. Larger and smaller gap widths: gap width to single deck ratios of $0.5,0.75$ and 1.0 were tested in this study. Larger and smaller gap widths can also be studied to help in optimizing the gap width for twin-deck bridges considering the aerodynamic and aeroelastic responses.

\subsection{Partial Turbulence Simulation}

In this study, reduced turbulence intensity which is defined based on the Partial Turbulence Simulation method was used to relate some of the aerodynamic features of the studied cross section to the approaching flow turbulence characteristics. The validity of the given hypothesis needs to be evaluated for a wider range of reduced turbulence intensities and for a wider range of aerodynamic and aeroelastic features including the flutter wind speed and amplitudes of vibration due to vortex induced vibrations. Whether similar behavior exists for the self-excited forces mentioned here is an issue worthy of further study.

\subsection{Buffeting Loads}

In this study, the basic parameters important in the calculation of buffeting loads from the spectral approach were measured and compared for different turbulence intensities and turbulence integral length scale to bridge width ratios. The minimum spanwise separation length considered here was large compared to the turbulence integral 
length scale of the approaching flow. In order to better capture the spatial distribution of wind-induced loads and compare the results to spatial distribution of the approaching flow, it is recommended that tests be performed for a larger number of separation lengths with a wider range of lengths including very short to very large separation lengths. Also, the tests could be repeated for dynamic condition in which the motion-induced effects on buffeting loads can be captured. This would help to identify differences between stationary and oscillating body buffeting forces. 
VITA

\section{RAMTIN KARGARMOAKHAR}

Born, Tehran, Iran

2003-2007

B.Sc., Civil Engineering

Amirkabir University of Technology

Tehran, Iran

2007-2010

M.Sc., Civil Engineering (Structural)

University of Tehran

Tehran, Iran

2010-2015

Research and Teaching Assistant

Florida International University

Miami, Florida

$2010-2015$

$\mathrm{PhD}$, Civil Engineering

Florida International University

Miami, Florida

\section{PUBLICATIONS AND PRESENTATIONS}

Ghassemieh, M., Kargarmoakhar, R. (2013). Response Modification Factor of Steel Frames Utilizing Shape Memory Alloys, Journal of Intelligent Material Systems and Structures, vol. 24, no. 10, pp. 1213-1225.

Kargarmoakhar, R., Chowdhury, A., Irwin, P. (2015). Reynolds Number Effects on Twin Box Girder Long Span Bridge Aerodynamics, Wind \& Structures, Accepted for Publication.

Kargarmoakhar, R., Chowdhury, A., Irwin, P. (2015). The Effects of Turbulence on the Pressure Distribution around a Twin Box Girder Bridge and the Resultant Aerodynamic Forces, Journal of Wind Engineering \& Industrial Aerodynamics, Submitted for Publication.

Kargarmoakhar, R., Chowdhury, A., Irwin, P. (2015). Effects of deck details on the twin deck bridge aerodynamics, Journal of Wind Engineering \& Industrial Aerodynamics, Submitted for Publication.

Mintz, B., Chowdhury, A., Mirmiran, A., Suksawang, N. and Kargarmoakhar, R. (2014) Design Procedures and Testing of a Super-Tile Roofing System, ASCE Journal of Composites for Construction, Submitted for Publication. 
Kargarmoakhar, R., Gan Chowdhury, A., Irwin, P. (June 2015). Effects of Gap Width on Twin-Deck Bridge Aerodynamics, 14th International Conference on Wind Engineering, Porto Alegre, Brazil, June 21-26, 2015, Accepted for presentation.

Kargarmoakhar, R., Chowdhury, A., Irwin, P. (2014). Reynolds Number effects on the Long Span Bridge Aerodynamics, Structures Congress, April 3-5, Boston, Massachusetts.

Baheru, T., Kargarmoakhar, R., Chowdhury, A., Lin, C. (2012). Large-scale measurements of the wind induced external convective heat transfer coefficient for building envelope, 3rd American Association for Wind Engineering Workshop, August 12-14, Hyannis, Massachusetts.

Ghassemieh, M., Kargarmoakhar, R., (2010). Response Modification Factor of Structural Steel Braced Frame Utilizing SMA, May 16-20, 2010, Pacific Grove, California. 